Supporting Information

\title{
Tertiary Alcohols as Radical Precursors for the Introduction of Tertiary Substituents into Heteroarenes
}

Spencer P. Pitre, Mikko Muuronen, Dmitry A. Fishman, and Larry E. Overman*

Department of Chemistry, University of California, Irvine, California 92697-2025, United States

Email: Larry E. Overman: leoverma@uci.edu

Table of Contents

$\begin{array}{ll}\text { A. Materials and Methods } & \text { S2 }\end{array}$

B. Picture of Photochemistry Set-Up $\quad$ S3

C. General Procedure for Photoredox-Catalyzed Minisci Reaction (GP1) S4

D. General Procedure for Thermal-Initiated Minisci Reaction (GP2) S5

E. Full Reaction Optimization for Photoredox-Catalyzed Minisci Reaction $\quad$ S6

F. Unoptimized Secondary Alkyl Oxalate Examples $\quad$ S8

G. Unsuccessful or Low Yielding Heterocycle Examples $\quad$ S11

H. Computational Analysis for the Minisci Reaction of Tertiary Radicals $\quad$ S12

I. Excited State Kinetic Quenching Studies $\quad$ S15

J. Determination of the Quantum Yield for the Photoredox-Catalyzed Minisci Reaction

K. Preparation of Starting Materials $\quad$ S23

L. Experimental Procedures for the Photoredox-Catalyzed Minisci Reaction $\quad$ S34

M. Experimental Procedures for the Thermal Minisci Reaction $\quad$ S69

$\begin{array}{ll}\text { N. Cyclic Voltammetry } & \text { S75 }\end{array}$

$\begin{array}{lr}\text { O. References } & \text { S77 }\end{array}$

$\begin{array}{lr}\text { P. NMR Spectra } & \text { S79 }\end{array}$ 


\section{A. Materials and Methods}

Unless stated otherwise, reactions were conducted in oven-dried glassware under an atmosphere of argon using anhydrous solvents (passed through activated alumina columns). All commercially available reagents were used as received. Extra dry DMSO was purchased from Acros Organics and used as received. $\operatorname{Ir}\left[\mathrm{dF}\left(\mathrm{CF}_{3}\right) \text { ppy }\right]_{2}(\mathrm{dtbbpy}) \mathrm{PF}_{6}$ was prepared according to literature procedures. ${ }^{1}$ Hemioxalate salts were prepared by methods described previously. ${ }^{2}$ Thin-layer chromatography (TLC) was conducted using E. Merck silica gel 50 F254 pre-coated plates $(0.25$ $\mathrm{mm}$ ) and visualized by exposure to UV light or potassium permanganate staining. Flash column chromatography was performed using a Biotage Isolera One equipped with ZIP columns, unless otherwise noted. ${ }^{1} \mathrm{H}$ NMR spectra were recorded at $600 \mathrm{MHz}$. Data for ${ }^{1} \mathrm{H}$ NMR spectra are reported as follows: chemical shift $(\delta \mathrm{ppm})$ multiplicity, coupling constant $(\mathrm{Hz})$ and integration. ${ }^{13} \mathrm{C}$ NMR spectra were recorded at $151 \mathrm{MHz}$. Data for ${ }^{13} \mathrm{C}$ NMR are reported in terms of chemical shift. NMR spectra are internally referenced to the residual solvent signals (note: $\mathrm{CDCl}_{3}$ referenced at $7.26 \mathrm{ppm}$ for ${ }^{1} \mathrm{H}$ NMR and $77.16 \mathrm{ppm}$ for ${ }^{13} \mathrm{C}$ NMR). IR spectra were recorded using an ATRFTIR spectrometer and are reported in terms of frequency of absorption $\left(\mathrm{cm}^{-1}\right)$. High resolution mass spectra were obtained from the UC Irvine Mass spectrometry facility. All photochemistry experiments were performed using two Kessil PR160 456 nm LED lamps purchased directly from Kessil. Dichloromethane is abbreviated as DCM. 


\section{B. Picture of Photochemistry Setup}

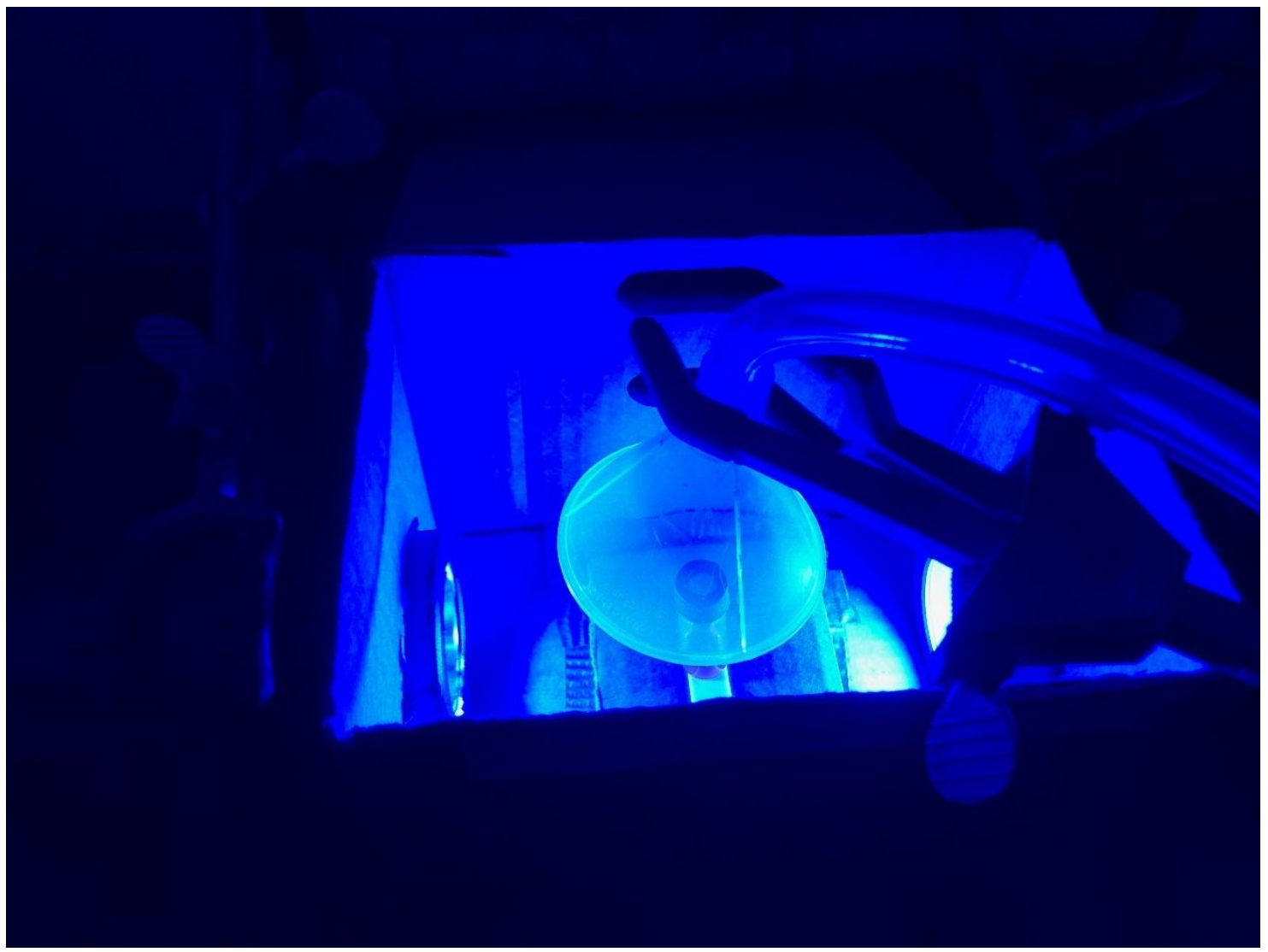

Figure S1. Picture of the photochemistry set-up employed for the photoredox-catalyzed Minisci reactions. 


\section{General Procedure for Photoredox-Catalyzed Minisci Reaction (GP1)}

An oven-dried one-dram vial equipped with a magnetic stir bar was charged with a hemioxalate salt (0.6 mmol, 1.2 equiv), $\operatorname{Ir}\left[\mathrm{dF}\left(\mathrm{CF}_{3}\right) \text { ppy }\right]_{2}(\mathrm{dtbbpy}) \mathrm{PF}_{6}(2.8 \mathrm{mg}, 0.0025 \mathrm{mmol}, 0.5 \mathrm{~mol} \%)$, $\left(\mathrm{NH}_{4}\right)_{2} \mathrm{~S}_{2} \mathrm{O}_{8}$ (171 mg, $0.75 \mathrm{mmol}, 1.5$ equiv) and a heterocycle (0.5 mmol, 1 equiv). Dry DMSO was added $(1 \mathrm{~mL}, 0.5 \mathrm{M})$, and the reaction mixture was degassed by sparging with argon for 10 min. The reaction mixture was then sonicated and irradiated with two Kessil PR160 456 nm LED lamps for $10 \mathrm{~min}$ to $6 \mathrm{~h}$. The reaction temperature was maintained at $30{ }^{\circ} \mathrm{C}$ by blowing air into the photochemistry setup. The reaction mixture was transferred into a separatory funnel and diluted with $25 \mathrm{~mL}$ of $\mathrm{EtO}_{2}$ and quenched with $25 \mathrm{~mL}$ of saturated $\mathrm{NaHCO}_{3(\mathrm{aq})}$. The $\mathrm{EtO}_{2}$ phase was washed with brine $(2 \times 25 \mathrm{~mL})$, and the combined aqueous phases were extracted with $\mathrm{EtO}_{2}(2 \times$ $25 \mathrm{~mL}$ ). The combined organic phases were dried with $\mathrm{MgSO}_{4}$ and concentrated. Purification was

performed by flash column chromatography using a Biotage Isolera One equipped with a $10 \mathrm{~g}$ Biotage ZIP column. 


\section{General Procedure for Thermal-Initiated Minisci Reaction (GP2)}

An oven-dried one-dram vial equipped with a magnetic stir bar was charged with a hemioxalate salt (0.6 mmol, 1.2 equiv), $\left(\mathrm{NH}_{4}\right)_{2} \mathrm{~S}_{2} \mathrm{O}_{8}(171 \mathrm{mg}, 0.75 \mathrm{mmol}, 1.5$ equiv) and a heterocycle $(0.5$ mmol, 1 equiv). Dry DMSO was added $(1 \mathrm{~mL}, 0.5 \mathrm{M})$, and the reaction mixture was degassed by sparging with argon for 10 min. The reaction mixture was sonicated and placed in a heating block that was preheated to $65^{\circ} \mathrm{C}$ where it was stirred for $10 \mathrm{~min}$. The reaction mixture was transferred into a separatory funnel and diluted with $25 \mathrm{~mL}$ of $\mathrm{EtO}_{2}$ and quenched with $25 \mathrm{~mL}$ of saturated $\mathrm{NaHCO}_{3(\mathrm{aq})}$. The $\mathrm{EtO}_{2}$ phase was washed with brine $(2 \times 25 \mathrm{~mL})$, and the combined aqueous phases

were extracted with $\mathrm{EtO}_{2}(2 \times 25 \mathrm{~mL})$. The combined organic phases were dried with $\mathrm{MgSO}_{4}$ and concentrated. Purification was performed by flash column chromatography using a Biotage Isolera One equipped with a $10 \mathrm{~g}$ Biotage ZIP column. 


\section{E. Full Reaction Optimization for Photoredox-Catalyzed Minisci Reaction}

Table S1. Optimization and control reactions for the tert-alkylation of lepidine.
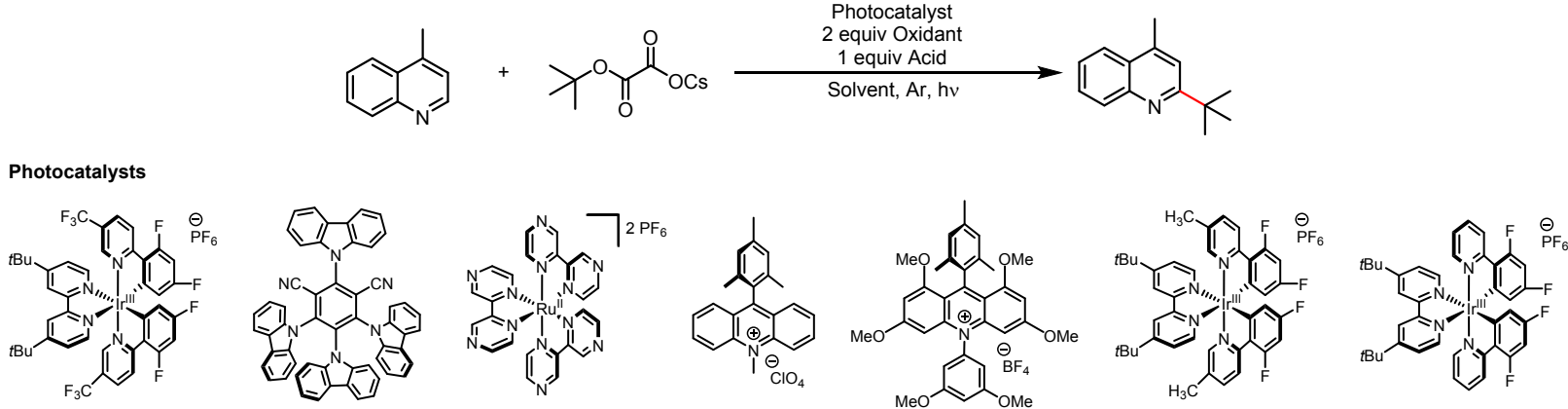

\begin{tabular}{|c|c|}
\hline $\begin{array}{c}\text { mmol } \\
\text { Lepidine }\end{array}$ & $\begin{array}{r}\text { Equiv } \\
\text { Oxalat }\end{array}$ \\
\hline $0.3 \mathrm{mmol}$ & 1.1 \\
\hline $0.3 \mathrm{mmol}$ & 1.1 \\
\hline $0.3 \mathrm{mmol}$ & 1.1 \\
\hline $0.3 \mathrm{mmol}$ & 1.1 \\
\hline $0.3 \mathrm{mmol}$ & 1.1 \\
\hline $0.3 \mathrm{mmol}$ & 1.1 \\
\hline $0.3 \mathrm{mmol}$ & 1.1 \\
\hline $0.3 \mathrm{mmol}$ & 1.1 \\
\hline $0.3 \mathrm{mmol}$ & 1.1 \\
\hline $0.3 \mathrm{mmol}$ & 1.1 \\
\hline $0.3 \mathrm{mmol}$ & 1.1 \\
\hline $0.3 \mathrm{mmol}$ & 1.1 \\
\hline $0.3 \mathrm{mmol}$ & 1.1 \\
\hline $0.3 \mathrm{mmol}$ & 1.1 \\
\hline $0.3 \mathrm{mmol}$ & 1.1 \\
\hline $0.3 \mathrm{mmol}$ & 1.1 \\
\hline $0.3 \mathrm{mmol}$ & 1.1 \\
\hline $0.3 \mathrm{mmol}$ & 1.1 \\
\hline $0.5 \mathrm{mmol}$ & 1.1 \\
\hline $0.5 \mathrm{mmol}$ & 1.1 \\
\hline $0.5 \mathrm{mmol}$ & 1.1 \\
\hline $0.5 \mathrm{mmol}$ & 1.1 \\
\hline $0.5 \mathrm{mmol}$ & 1.1 \\
\hline $0.5 \mathrm{mmol}$ & 1.1 \\
\hline $0.5 \mathrm{mmol}$ & 1.1 \\
\hline $0.25 \mathrm{mmol}$ & 1.1 \\
\hline $0.25 \mathrm{mmol}$ & 1.1 \\
\hline $0.25 \mathrm{mmol}$ & 1.1 \\
\hline $0.25 \mathrm{mmol}$ & 1.1 \\
\hline $0.25 \mathrm{mmol}$ & 1.1 \\
\hline $0.25 \mathrm{mmol}$ & 1.1 \\
\hline $0.25 \mathrm{mmol}$ & 1.1 \\
\hline $0.25 \mathrm{mmol}$ & 1.1 \\
\hline $0.25 \mathrm{mmol}$ & 1.1 \\
\hline $0.25 \mathrm{mmol}$ & 1.1 \\
\hline $0.25 \mathrm{mmol}$ & 1.1 \\
\hline $0.25 \mathrm{mmol}$ & 1.1 \\
\hline $0.25 \mathrm{mmol}$ & 1.0 \\
\hline $0.25 \mathrm{mmol}$ & 1.2 \\
\hline $0.25 \mathrm{mmol}$ & 1.2 \\
\hline $0.25 \mathrm{mmol}$ & 1.2 \\
\hline $0.25 \mathrm{mmol}$ & 1.2 \\
\hline $0.25 \mathrm{mmol}$ & 1.2 \\
\hline $0.25 \mathrm{mmol}$ & 1.2 \\
\hline $0.25 \mathrm{mmol}$ & 1.2 \\
\hline $0.25 \mathrm{mmol}$ & 1.2 \\
\hline $0.25 \mathrm{mmol}$ & 1.2 \\
\hline
\end{tabular}

II

III

IV

$\mathrm{V}$

$\mathrm{V}$

VII

\begin{tabular}{|c|c|c|c|c|c|c|}
\hline PC (mol\%) & Oxidant & Acid & Solvent ([M]) & $\mathbf{T}$ & Time & $\%$ Yield \\
\hline I (1 mol\%) & $\mathrm{Na}_{2} \mathrm{~S}_{2} \mathrm{O}_{8}$ & None & $\operatorname{DMF}(0.1 \mathrm{M})$ & $60^{\circ} \mathrm{C}$ & $18 \mathrm{~h}$ & $5 \%$ \\
\hline I (1 mol\%) & $\mathrm{K}_{2} \mathrm{~S}_{2} \mathrm{O}_{8}$ & None & $\operatorname{DMF}(0.1 \mathrm{M})$ & $60^{\circ} \mathrm{C}$ & $18 \mathrm{~h}$ & $4 \%$ \\
\hline I (1 mol\%) & $\left(\mathrm{NH}_{4}\right)_{2} \mathrm{~S}_{2} \mathrm{O}_{8}$ & None & $\operatorname{DMF}(0.1 \mathrm{M})$ & $60^{\circ} \mathrm{C}$ & $18 \mathrm{~h}$ & $11 \%$ \\
\hline II (1 mol \%) & $\left(\mathrm{NH}_{4}\right)_{2} \mathrm{~S}_{2} \mathrm{O}_{8}$ & None & DMF (0.1 M) & $60^{\circ} \mathrm{C}$ & $18 \mathrm{~h}$ & $5 \%$ \\
\hline III ( $1 \mathrm{~mol} \%)$ & $\left(\mathrm{NH}_{4}\right)_{2} \mathrm{~S}_{2} \mathrm{O}_{8}$ & None & DMF (0.1 M) & $60^{\circ} \mathrm{C}$ & $18 \mathrm{~h}$ & Trace \\
\hline IV (1 mol \%) & $\left(\mathrm{NH}_{4}\right)_{2} \mathrm{~S}_{2} \mathrm{O}_{8}$ & None & $\operatorname{DMF}(0.1 \mathrm{M})$ & $60^{\circ} \mathrm{C}$ & $18 \mathrm{~h}$ & Trace \\
\hline $\mathrm{V}(1 \mathrm{~mol} \%)$ & $\left(\mathrm{NH}_{4}\right)_{2} \mathrm{~S}_{2} \mathrm{O}_{8}$ & None & $\operatorname{DMF}(0.1 \mathrm{M})$ & $60^{\circ} \mathrm{C}$ & $18 \mathrm{~h}$ & $4 \%$ \\
\hline I (1 mol\%) & $\left(\mathrm{NH}_{4}\right)_{2} \mathrm{~S}_{2} \mathrm{O}_{8}$ & $\mathrm{HCl}(1 \mathrm{~N})$ & $\operatorname{DMF}(0.1 \mathrm{M})$ & $60^{\circ} \mathrm{C}$ & $18 \mathrm{~h}$ & $5 \%$ \\
\hline I (1 mol\%) & $\left(\mathrm{NH}_{4}\right)_{2} \mathrm{~S}_{2} \mathrm{O}_{8}$ & $\mathrm{AcOH}$ & $\operatorname{DMF}(0.1 \mathrm{M})$ & $60^{\circ} \mathrm{C}$ & $18 \mathrm{~h}$ & - \\
\hline I (1 mol\%) & $\left(\mathrm{NH}_{4}\right)_{2} \mathrm{~S}_{2} \mathrm{O}_{8}$ & Formic & $\operatorname{DMF}(0.1 \mathrm{M})$ & $60^{\circ} \mathrm{C}$ & $18 \mathrm{~h}$ & - \\
\hline I (1 mol\%) & $\left(\mathrm{NH}_{4}\right)_{2} \mathrm{~S}_{2} \mathrm{O}_{8}$ & TFA & $\operatorname{DMF}(0.1 \mathrm{M})$ & $60^{\circ} \mathrm{C}$ & $18 \mathrm{~h}$ & $12 \%$ \\
\hline I (1 mol\%) & $\left(\mathrm{NH}_{4}\right)_{2} \mathrm{~S}_{2} \mathrm{O}_{8}$ & TFA & 3:1 DME:DMF (0.1 M) & $60^{\circ} \mathrm{C}$ & $18 \mathrm{~h}$ & $7 \%$ \\
\hline I (1 mol\%) & $\left(\mathrm{NH}_{4}\right)_{2} \mathrm{~S}_{2} \mathrm{O}_{8}$ & TFA & $\operatorname{DME}(0.1 \mathrm{M})$ & $60^{\circ} \mathrm{C}$ & $18 \mathrm{~h}$ & $5 \%$ \\
\hline I (1 mol\%) & $\left(\mathrm{NH}_{4}\right)_{2} \mathrm{~S}_{2} \mathrm{O}_{8}$ & TFA & THF (0.1 M) & $60^{\circ} \mathrm{C}$ & $18 \mathrm{~h}$ & $3 \%$ \\
\hline I (1 mol\%) & $\left(\mathrm{NH}_{4}\right)_{2} \mathrm{~S}_{2} \mathrm{O}_{8}$ & TFA & $\mathrm{MeCN}(0.1 \mathrm{M})$ & $60^{\circ} \mathrm{C}$ & $18 \mathrm{~h}$ & $10 \%$ \\
\hline I (1 mol\%) & $\left(\mathrm{NH}_{4}\right)_{2} \mathrm{~S}_{2} \mathrm{O}_{8}$ & TFA & $4: 1 \mathrm{MeCN}: \mathrm{H}_{2} \mathrm{O}(0.1 \mathrm{M})$ & $60^{\circ} \mathrm{C}$ & $18 \mathrm{~h}$ & $11 \%$ \\
\hline I (1 mol\%) & $\left(\mathrm{NH}_{4}\right)_{2} \mathrm{~S}_{2} \mathrm{O}_{8}$ & TFA & $\mathrm{MeOH}(0.1 \mathrm{M})$ & $60^{\circ} \mathrm{C}$ & $18 \mathrm{~h}$ & - \\
\hline I (1 mol\%) & $\left(\mathrm{NH}_{4}\right)_{2} \mathrm{~S}_{2} \mathrm{O}_{8}$ & TFA & $\mathrm{MeCN}(0.25 \mathrm{M})$ & $60^{\circ} \mathrm{C}$ & $18 \mathrm{~h}$ & $5 \%$ \\
\hline I (1 mol\%) & $\left(\mathrm{NH}_{4}\right)_{2} \mathrm{~S}_{2} \mathrm{O}_{8}$ & TFA & $\mathrm{MeCN}(0.5 \mathrm{M})$ & $60^{\circ} \mathrm{C}$ & $18 \mathrm{~h}$ & $21 \%$ \\
\hline I (3 mol\%) & $\left(\mathrm{NH}_{4}\right)_{2} \mathrm{~S}_{2} \mathrm{O}_{8}$ & TFA & $\mathrm{MeCN}(0.5 \mathrm{M})$ & $60^{\circ} \mathrm{C}$ & $18 \mathrm{~h}$ & $31 \%$ \\
\hline I (5 mol\%) & $\left(\mathrm{NH}_{4}\right)_{2} \mathrm{~S}_{2} \mathrm{O}_{8}$ & TFA & $\mathrm{MeCN}(0.5 \mathrm{M})$ & $60^{\circ} \mathrm{C}$ & $18 \mathrm{~h}$ & $47 \%$ \\
\hline I (5 mol\%) & $\left(\mathrm{NH}_{4}\right)_{2} \mathrm{~S}_{2} \mathrm{O}_{8}$ & $\mathrm{HCl}(1 \mathrm{~N})$ & $\mathrm{MeCN}(0.5 \mathrm{M})$ & $60^{\circ} \mathrm{C}$ & $18 \mathrm{~h}$ & $55 \%$ \\
\hline I (5 mol\%) & $\left(\mathrm{NH}_{4}\right)_{2} \mathrm{~S}_{2} \mathrm{O}_{8}$ & $\mathrm{H}_{2} \mathrm{SO}_{4}$ & $\mathrm{MeCN}(0.5 \mathrm{M})$ & $60^{\circ} \mathrm{C}$ & $18 \mathrm{~h}$ & $17 \%$ \\
\hline I (5 mol\%) & $\left(\mathrm{NH}_{4}\right)_{2} \mathrm{~S}_{2} \mathrm{O}_{8}$ & CSA & $\operatorname{MeCN}(0.5 \mathrm{M})$ & $60^{\circ} \mathrm{C}$ & $18 \mathrm{~h}$ & $35 \%$ \\
\hline I (5 mol\%) & $\left(\mathrm{NH}_{4}\right)_{2} \mathrm{~S}_{2} \mathrm{O}_{8}$ & PTSA & $\operatorname{MeCN}(0.5 \mathrm{M})$ & $60^{\circ} \mathrm{C}$ & $18 \mathrm{~h}$ & $53 \%$ \\
\hline I (5 mol\%) & $\left(\mathrm{NH}_{4}\right)_{2} \mathrm{~S}_{2} \mathrm{O}_{8}$ & $\mathrm{HCl}(1 \mathrm{~N})$ & $\operatorname{DCE}(0.5 \mathrm{M})$ & $60^{\circ} \mathrm{C}$ & $18 \mathrm{~h}$ & $34 \%$ \\
\hline I (5 mol\%) & $\left(\mathrm{NH}_{4}\right)_{2} \mathrm{~S}_{2} \mathrm{O}_{8}$ & $\mathrm{HCl}(1 \mathrm{~N})$ & Dioxane $(0.5 \mathrm{M})$ & $60^{\circ} \mathrm{C}$ & $18 \mathrm{~h}$ & $7 \%$ \\
\hline I (5 mol\%) & $\left(\mathrm{NH}_{4}\right)_{2} \mathrm{~S}_{2} \mathrm{O}_{8}$ & $\mathrm{HCl}(1 \mathrm{~N})$ & THF (0.5 M) & $60^{\circ} \mathrm{C}$ & $18 \mathrm{~h}$ & $14 \%$ \\
\hline $\mathrm{I}(5 \mathrm{~mol} \%)$ & $\left(\mathrm{NH}_{4}\right)_{2} \mathrm{~S}_{2} \mathrm{O}_{8}$ & $\mathrm{HCl}(1 \mathrm{~N})$ & $\operatorname{DME}(0.5 \mathrm{M})$ & $60^{\circ} \mathrm{C}$ & $18 \mathrm{~h}$ & $21 \%$ \\
\hline I (5 mol\%) & $\left(\mathrm{NH}_{4}\right)_{2} \mathrm{~S}_{2} \mathrm{O}_{8}$ & $\mathrm{HCl}(1 \mathrm{~N})$ & DMSO (0.5 M) & $60^{\circ} \mathrm{C}$ & $18 \mathrm{~h}$ & $77 \%$ \\
\hline I (3 mol\%) & $\left(\mathrm{NH}_{4}\right)_{2} \mathrm{~S}_{2} \mathrm{O}_{8}$ & $\mathrm{HCl}(1 \mathrm{~N})$ & $\operatorname{DMSO}(0.5 \mathrm{M})$ & $60^{\circ} \mathrm{C}$ & $18 \mathrm{~h}$ & $78 \%$ \\
\hline I (3 mol\%) & $\left(\mathrm{NH}_{4}\right)_{2} \mathrm{~S}_{2} \mathrm{O}_{8}$ & $\mathrm{HCl}(1 \mathrm{~N})$ & $\operatorname{DMSO}(0.5 \mathrm{M})$ & $60^{\circ} \mathrm{C}$ & $1 \mathrm{~h}$ & $79 \%$ \\
\hline I (3 mol\%) & $\left(\mathrm{NH}_{4}\right)_{2} \mathrm{~S}_{2} \mathrm{O}_{8}$ & $\mathrm{HCl}(1 \mathrm{~N})$ & $\operatorname{DMSO}(0.5 \mathrm{M})$ & $60^{\circ} \mathrm{C}$ & $1 \mathrm{~h}$ & $90 \% *$ \\
\hline I (3 mol\%) & $\left(\mathrm{NH}_{4}\right)_{2} \mathrm{~S}_{2} \mathrm{O}_{8}$ & $\mathrm{HCl}(1 \mathrm{~N})$ & $\operatorname{DMSO}(0.5 \mathrm{M})$ & $60^{\circ} \mathrm{C}$ & $15 \mathrm{~min}$ & $89 \%$ \\
\hline I (3 mol\%) & $\left(\mathrm{NH}_{4}\right)_{2} \mathrm{~S}_{2} \mathrm{O}_{8}$ & $\mathrm{HCl}(1 \mathrm{~N})$ & $\operatorname{DMSO}(0.5 \mathrm{M})$ & $60^{\circ} \mathrm{C}$ & $10 \mathrm{~min}$ & $90 \%$ \\
\hline I (2 mol\%) & $\left(\mathrm{NH}_{4}\right)_{2} \mathrm{~S}_{2} \mathrm{O}_{8}$ & $\mathrm{HCl}(1 \mathrm{~N})$ & $\operatorname{DMSO}(0.5 \mathrm{M})$ & $60^{\circ} \mathrm{C}$ & $10 \mathrm{~min}$ & $91 \%$ \\
\hline I (1 mol\%) & $\left(\mathrm{NH}_{4}\right)_{2} \mathrm{~S}_{2} \mathrm{O}_{8}$ & $\mathrm{HCl}(1 \mathrm{~N})$ & $\operatorname{DMSO}(0.5 \mathrm{M})$ & $60^{\circ} \mathrm{C}$ & $10 \mathrm{~min}$ & $90 \%$ \\
\hline I (1 mol\%) & $\left(\mathrm{NH}_{4}\right)_{2} \mathrm{~S}_{2} \mathrm{O}_{8}$ & $\mathrm{HCl}(1 \mathrm{~N})$ & $\operatorname{DMSO}(0.5 \mathrm{M})$ & $60^{\circ} \mathrm{C}$ & $10 \mathrm{~min}$ & $83 \%$ \\
\hline I (1 mol\%) & $\left(\mathrm{NH}_{4}\right)_{2} \mathrm{~S}_{2} \mathrm{O}_{8}$ & $\mathrm{HCl}(1 \mathrm{~N})$ & $\operatorname{DMSO}(0.5 \mathrm{M})$ & $60^{\circ} \mathrm{C}$ & $10 \mathrm{~min}$ & $94 \%$ \\
\hline $\mathrm{I}(0.5 \mathrm{~mol} \%)$ & $\left(\mathrm{NH}_{4}\right)_{2} \mathrm{~S}_{2} \mathrm{O}_{8}$ & $\mathrm{HCl}(1 \mathrm{~N})$ & $\operatorname{DMSO}(0.5 \mathrm{M})$ & $60^{\circ} \mathrm{C}$ & $10 \mathrm{~min}$ & $95 \%$ \\
\hline None & $\left(\mathrm{NH}_{4}\right)_{2} \mathrm{~S}_{2} \mathrm{O}_{8}$ & $\mathrm{HCl}(1 \mathrm{~N})$ & $\operatorname{DMSO}(0.5 \mathrm{M})$ & $60^{\circ} \mathrm{C}$ & $10 \mathrm{~min}$ & $94 \%$ \\
\hline None & $\left(\mathrm{NH}_{4}\right)_{2} \mathrm{~S}_{2} \mathrm{O}_{8}$ & $\mathrm{HCl}(1 \mathrm{~N})$ & $\operatorname{DMSO}(0.5 \mathrm{M})$ & $30^{\circ} \mathrm{C}$ & $10 \mathrm{~min}$ & $6 \%$ \\
\hline $\mathrm{I}(0.5 \mathrm{~mol} \%)$ & $\left(\mathrm{NH}_{4}\right)_{2} \mathrm{~S}_{2} \mathrm{O}_{8}$ & $\mathrm{HCl}(1 \mathrm{~N})$ & $\operatorname{DMSO}(0.5 \mathrm{M})$ & $30^{\circ} \mathrm{C}$ & $10 \mathrm{~min}$ & $94 \%$ \\
\hline VI (0.5 mol\%) & $\left(\mathrm{NH}_{4}\right)_{2} \mathrm{~S}_{2} \mathrm{O}_{8}$ & $\mathrm{HCl}(1 \mathrm{~N})$ & $\operatorname{DMSO}(0.5 \mathrm{M})$ & $30^{\circ} \mathrm{C}$ & $10 \mathrm{~min}$ & $89 \%$ \\
\hline VII (0.5 mol\%) & $\left(\mathrm{NH}_{4}\right)_{2} \mathrm{~S}_{2} \mathrm{O}_{8}$ & $\mathrm{HCl}(1 \mathrm{~N})$ & $\operatorname{DMSO}(0.5 \mathrm{M})$ & $30^{\circ} \mathrm{C}$ & $10 \mathrm{~min}$ & $91 \%$ \\
\hline II (0.5 mol\%) & $\left(\mathrm{NH}_{4}\right)_{2} \mathrm{~S}_{2} \mathrm{O}_{8}$ & $\mathrm{HCl}(1 \mathrm{~N})$ & $\operatorname{DMSO}(0.5 \mathrm{M})$ & $30^{\circ} \mathrm{C}$ & $10 \mathrm{~min}$ & $57 \%$ \\
\hline $\mathrm{I}(0.5 \mathrm{~mol} \%)$ & None & $\mathrm{HCl}(1 \mathrm{~N})$ & $\operatorname{DMSO}(0.5 \mathrm{M})$ & $30^{\circ} \mathrm{C}$ & $10 \mathrm{~min}$ & - \\
\hline
\end{tabular}




\begin{tabular}{ccccccccc}
$0.25 \mathrm{mmol}$ & 1.2 & $\mathrm{I}(0.5 \mathrm{~mol} \%)$ & $\left(\mathrm{NH}_{4}\right)_{2} \mathrm{~S}_{2} \mathrm{O}_{8}$ & None & DMSO $(0.5 \mathrm{M})$ & $30{ }^{\circ} \mathrm{C}$ & $10 \mathrm{~min}$ & $93 \%$ \\
$0.25 \mathrm{mmol}$ & 1.2 & $\mathrm{I}(0.5 \mathrm{~mol} \%)$ & $\left(\mathrm{NH}_{4}\right)_{2} \mathrm{~S}_{2} \mathrm{O}_{8}{ }^{\mathrm{a}}$ & None & DMSO $(0.5 \mathrm{M})$ & $30{ }^{\circ} \mathrm{C}$ & $10 \mathrm{~min}$ & $81 \%$ \\
$0.25 \mathrm{mmol}$ & 1.2 & $\mathrm{I}(0.5 \mathrm{~mol} \%)$ & $\left(\mathrm{NH}_{4}\right)_{2} \mathrm{~S}_{2} \mathrm{O}_{8}{ }^{\mathrm{b}}$ & None & DMSO $(0.5 \mathrm{M})$ & $30{ }^{\circ} \mathrm{C}$ & $10 \mathrm{~min}$ & $90 \%$ \\
$0.25 \mathrm{mmol}$ & 1.2 & None & $\left(\mathrm{NH}_{4}\right)_{2} \mathrm{~S}_{2} \mathrm{O}_{8}{ }^{\mathrm{b}}$ & None & DMSO $(0.5 \mathrm{M})$ & $30{ }^{\circ} \mathrm{C}$ & $10 \mathrm{~min}$ & - \\
$0.25 \mathrm{mmol}$ & 1.2 & $\mathrm{I}(0.5 \mathrm{~mol} \%)$ & $\left(\mathrm{NH}_{4}\right)_{2} \mathrm{~S}_{2} \mathrm{O}_{8}{ }^{\mathrm{b}}$ & None & DMSO $(0.5 \mathrm{M})$ & $30^{\circ} \mathrm{C}$ & $10 \mathrm{~min}$ & $89 \%^{\mathrm{c}}$ \\
$0.5 \mathrm{mmol}$ & 1.2 & $\mathrm{I}(0.5 \mathrm{~mol} \%)$ & $\left(\mathrm{NH}_{4}\right)_{2} \mathrm{~S}_{2} \mathrm{O}_{8}{ }^{\mathrm{b}}$ & None & DMSO $(0.5 \mathrm{M})$ & $30^{\circ} \mathrm{C}$ & $10 \mathrm{~min}$ & $94 \%$ \\
\hline *At this point, a new work-up procedure was put in place. ${ }^{\mathrm{a}} 1.0$ equiv. of $\left(\mathrm{NH}_{4}\right)_{2} \mathrm{~S}_{2} \mathrm{O}_{8}$ was used. ${ }^{\mathrm{b}} 1.5$ equiv. of $\left(\mathrm{NH}_{4}\right)_{2} \mathrm{~S}_{2} \mathrm{O}_{8}$ was used. ${ }^{\mathrm{c}}$ Not degassed.
\end{tabular}




\section{F. Unoptimized Secondary Alkyl Oxalate Examples}

Although the focus of our studies, the reaction of a few secondary hemioxalate salts were studied, but not optimized.

Table S2. Scope of unoptimized secondary alkyl oxalate examples.

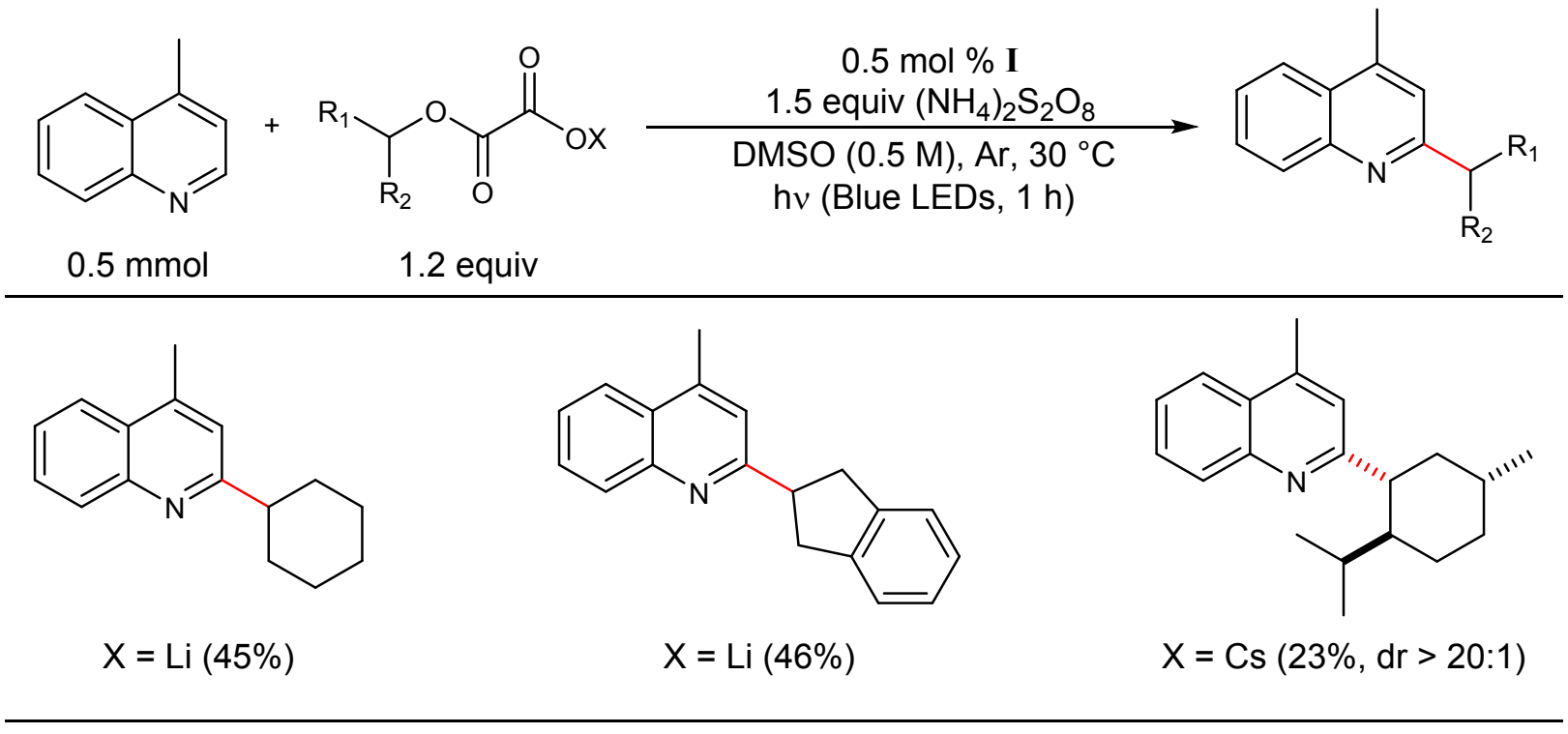<smiles>Cc1cc(C2CCCCC2)nc2ccccc12</smiles>

Prepared according to GP1 from lepidine ( $0.5 \mathrm{mmol}, 66 \mu \mathrm{L}, 1$ equiv), lithium 2-(cyclohexyloxy)2-oxoacetate (107 mg, $0.6 \mathrm{mmol}, 1.2$ equiv), $\operatorname{Ir}\left[\mathrm{dF}\left(\mathrm{CF}_{3}\right) \mathrm{ppy}\right]_{2}(\mathrm{dtbbpy}) \mathrm{PF}_{6}(2.8 \mathrm{mg}, 0.0025 \mathrm{mmol}$, $0.5 \mathrm{~mol} \%)$ and $\left(\mathrm{NH}_{4}\right)_{2} \mathrm{~S}_{2} \mathrm{O}_{8}(171 \mathrm{mg}, 0.75 \mathrm{mmol}, 1.5$ equiv) in $1 \mathrm{~mL}$ of dry DMSO. The reaction was irradiated with two $30 \mathrm{~W}$ Kessil blue LED lamps for $1 \mathrm{~h}$ at $30^{\circ} \mathrm{C}$. Purified by flash column chromatography (Hex $\rightarrow$ 9:1 Hex:EtOAc) to afford the desired compound as a clear oil in 45\% yield (62 mg).

${ }^{1}$ H NMR: $\left(600 \mathrm{MHz}, \mathrm{CDCl}_{3}\right) \delta 8.04(\mathrm{~d}, J=8.4 \mathrm{~Hz}, 1 \mathrm{H}), 7.94(\mathrm{~d}, J=8.2 \mathrm{~Hz}, 1 \mathrm{H}), 7.67-7.65(\mathrm{~m}, 1 \mathrm{H})$, 7.50-7.47 (m, 1H), $7.16(\mathrm{~m}, 1 \mathrm{H}), 2.87(\mathrm{tt}, J=12.1,3.4 \mathrm{~Hz}, 1 \mathrm{H}), 2.68(\mathrm{~s}, 3 \mathrm{H}), 2.04-1.99(\mathrm{~m}, 2 \mathrm{H})$, 
1.89 (dt, $J=13.2,3.1 \mathrm{~Hz}, \mathrm{H}), 1.82-1.76(\mathrm{~m}, 1 \mathrm{H}), 1.63$ (qd, $J=12.6,3.2 \mathrm{~Hz}, 2 \mathrm{H}), 1.47$ (qt, $J=12.9$, $3.3 \mathrm{~Hz}, 2 \mathrm{H}), 1.34$ (qt, $J=12.9,3.6 \mathrm{~Hz}, 1 \mathrm{H}) .{ }^{13} \mathbf{C}$ NMR: $\left(151 \mathrm{MHz}, \mathrm{CDCl}_{3}\right) \delta 166.7,147.8,144.3$, 129.7, 129.0, 127.2, 125.5, 123.7, 120.4, 47.8, 33.0, 26.7, 26.3, 19.0. Rf: 0.42 (8:1 Hex:EtOAc). Reference: Chem. Sci. 2016, 7, 4754.

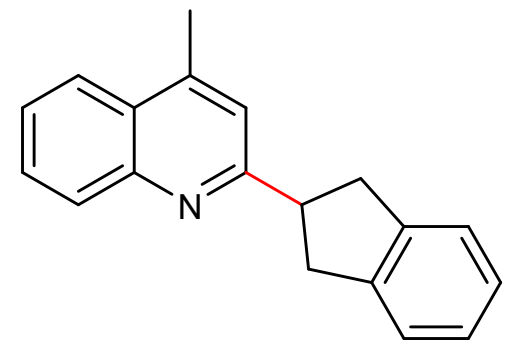

Prepared according to GP1 from lepidine ( $0.5 \mathrm{mmol}, 66 \mu \mathrm{L}, 1$ equiv), lithium 2-((2,3-dihydro-1Hinden-2-yl)oxy)-2-oxoacetate (127 mg, $0.6 \mathrm{mmol}, 1.2$ equiv), $\operatorname{Ir}\left[\mathrm{dF}\left(\mathrm{CF}_{3}\right) \mathrm{ppy}\right]_{2}(\mathrm{dtbbpy}) \mathrm{PF}_{6}(2.8$ $\mathrm{mg}, 0.0025 \mathrm{mmol}, 0.5 \mathrm{~mol} \%)$ and $\left(\mathrm{NH}_{4}\right)_{2} \mathrm{~S}_{2} \mathrm{O}_{8}(171 \mathrm{mg}, 0.75 \mathrm{mmol}, 1.5$ equiv) in $1 \mathrm{~mL}$ of dry DMSO. The reaction was irradiated with two $30 \mathrm{~W}$ Kessil blue LED lamps for $1 \mathrm{~h}$ at $30{ }^{\circ} \mathrm{C}$. Purified by flash column chromatography (Hex $\rightarrow$ 9:1 Hex:EtOAc) to afford the desired compound as a clear oil in $46 \%$ yield $(60 \mathrm{mg})$.

${ }^{1}$ H NMR: $\left.\left(600 \mathrm{MHz}, \mathrm{CDCl}_{3}\right) \delta 8.06(\mathrm{~d}, J=7.9 \mathrm{~Hz}, 1 \mathrm{H}), 7.96, \mathrm{dd}, J=8.3,0.9 \mathrm{~Hz}, 1 \mathrm{H}\right), 7.70-7.67$ (m, 1H), 7.53-7.51 (m, 1H), 7.29-7.27 (m, 2H), 7.21-7.19 (m, 3H), 4.03 (p, J=8.7, 1H), 3.48 (dd, $J=15.6,8.7 \mathrm{~Hz}, 2 \mathrm{H}), 3.40(\mathrm{dd}, J=15.8,8.7 \mathrm{~Hz}, 2 \mathrm{H}), 2.67(\mathrm{~s}, 3 \mathrm{H}) .{ }^{13} \mathbf{C}$ NMR: $\left(151 \mathrm{MHz}, \mathrm{CDCl}_{3}\right) \delta$ 164.8, 147.7, 144.7, 143.0, 129.7, 129.2, 127.2, 126.6, 125.8, 124.6, 123.7, 120.7, 48.1, 39.9, 19.0. Rf: 0.42 (8:1 Hex:EtOAc). Reference: Org Lett. 2018, 20, 4686. 


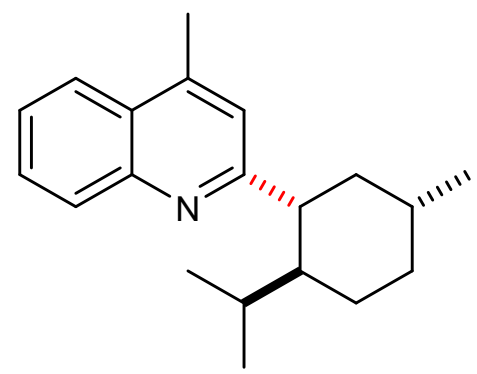

Prepared according to GP1 from lepidine $(0.5 \mathrm{mmol}, 66 \mu \mathrm{L}, 1$ equiv), cesium $2-(((1 S, 2 R, 5 S)-2-$ isopropyl-5-methylcyclohexyl)oxy)-2-oxoacetate ${ }^{2} \quad(216 \quad \mathrm{mg}, \quad 0.6 \quad \mathrm{mmol}, \quad 1.2$ equiv), $\operatorname{Ir}\left[\mathrm{dF}\left(\mathrm{CF}_{3}\right) \text { ppy }\right]_{2}(\mathrm{dtbbpy}) \mathrm{PF}_{6}(2.8 \mathrm{mg}, 0.0025 \mathrm{mmol}, 0.5 \mathrm{~mol} \%)$ and $\left(\mathrm{NH}_{4}\right)_{2} \mathrm{~S}_{2} \mathrm{O}_{8}(171 \mathrm{mg}, 0.75$ mmol, 1.5 equiv) in $1 \mathrm{~mL}$ of dry DMSO. The reaction was irradiated with two $30 \mathrm{~W}$ Kessil blue LED lamps for $1 \mathrm{~h}$ at $30^{\circ} \mathrm{C}$. Purified by flash column chromatography (Hex $\rightarrow$ 9:1 Hex:EtOAc) to afford the desired compound as a clear oil in $23 \%$ yield (32 mg).

1H NMR: $\left(600 \mathrm{MHz}, \mathrm{CDCl}_{3}\right) \delta 8.05(\mathrm{~d}, J=8.3 \mathrm{~Hz}, 1 \mathrm{H}), 7.94(\mathrm{~d}, J=8.3 \mathrm{~Hz}, 1 \mathrm{H}), 7.68-7.64(\mathrm{~m}, 1 \mathrm{H})$, 7.51-7.47 (m, 1H), $7.12(\mathrm{~s}, 1 \mathrm{H}), 2.90-2.84(\mathrm{~m}, 1 \mathrm{H}), 2.68(\mathrm{~s}, 3 \mathrm{H}), 1.91-1.82(\mathrm{~m}, 2 \mathrm{H}), 1.82-1.74(\mathrm{~m}$, 2H), 1.61-1.52 (m, 1H), 1.40-1.34 (m, 1H), 1.33-1.18 (m, 2H), 1.14-1.06 (m, 1H), $0.91(\mathrm{~d}, J=6.6$ $\mathrm{Hz}, 3 \mathrm{H}), 0.82(\mathrm{~d}, J=7.0 \mathrm{~Hz}, 3 \mathrm{H}), 0.74(\mathrm{~d}, J=6.9 \mathrm{~Hz}, 3 \mathrm{H}) .{ }^{13} \mathbf{C}$ NMR: $\left(151 \mathrm{MHz}, \mathrm{CDCl}_{3}\right) \delta 166.0$, $147.9,144.1,129.7,128.9,127.1,125.4,123.7,120.7,51.0,46.6,43.5,35.3,33.1,28.3,24.7,22.7$, 21.6, 19.0, 15.8. Rf: 0.58 (8:1 Hex:EtOAc). Reference: Org Lett. 2018, 20, 4686. 


\section{G. Unsuccessful or Low Yielding Heterocycle Examples}

Table S3. Scope of unsuccessful or low yielding heterocycles for the photoredox-catalyzed Minisci reaction.

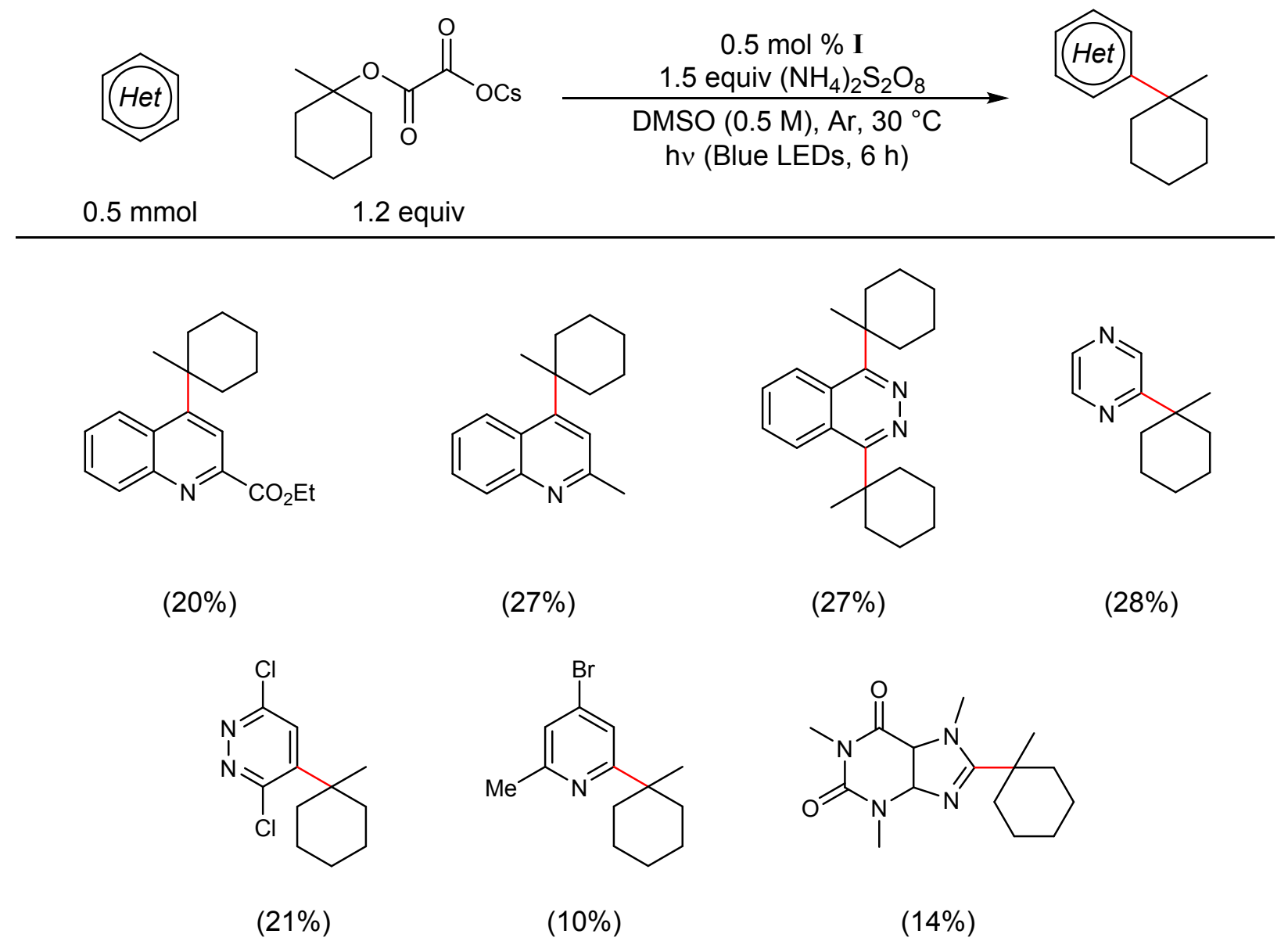

\section{Unsuccessful Substrates}<smiles>CC(=O)c1cc2ccccc2cn1</smiles><smiles>c1ccc2cnccc2c1</smiles><smiles>Brc1cncc2ccccc12</smiles><smiles>COC(=O)c1cc2ccccc2cn1</smiles><smiles>c1nc[nH]n1</smiles><smiles>[N+]#[X]c1ccnc(Cl)c1</smiles><smiles>Brc1cccc(Br)n1</smiles><smiles>c1ccc2[nH]ncc2c1</smiles><smiles>Cc1ccnc(Br)c1</smiles><smiles>c1ccc2ocnc2c1</smiles> 


\section{H. Computational Analysis for the Minisci Reaction of Tertiary Radicals}

Methods: All computations were performed using the Turbomole 7.2.1 program package. ${ }^{3}$ Default settings were used except finer integration grid of $m 4$ was employed. Structures were optimized in solution using the TPSSh-D3 ${ }^{4,5}$ functional with triple- $\zeta$ level def2-TZVP basis sets, ${ }^{6}$ and solvation effects were accounted for using the $\mathrm{COSMO}^{7}$ model with dielectric constant of infinity. The D3 dispersion corrections were used with BJ-damping. Computations were further sped-up by using the multipole-accelerated resolution-of-the-identity approximation (MARI-J) ${ }^{8}$ for the Coulomb term with the corresponding auxiliary basis sets. ${ }^{9}$ Harmonic vibrational frequencies were computed numerically to all optimized structures to verify the structure to be minima or transition state, and to calculate the chemical potentials (c.p.) at $298.15 \mathrm{~K}$ using the standard rigid-rotor harmonic oscillator approximation. The Gibbs free energies where then obtained according to $\mathrm{G}=$ $\mathrm{E}(0)+$ c.p.. Pictures of the structures were generated using Cylview. ${ }^{10}$

We previously identified that the transition states of the Giese reaction are strongly influenced by the self-interaction-error present in the common density functional approximations, i.e., the length of the forming $\mathrm{C}-\mathrm{C}$ bond in the transition state heavily depends on the functional used. ${ }^{11}$ TPSShD3 hybrid functional predicted the selectivities of the Giese reaction accurately and provided very similar transition state structures with the random phase approximation (RPA) method. Therefore, the transition state for the Giese reaction was optimized using same protocol, i.e., the forming C$\mathrm{C}$ bond was constrained during the optimization with $5 \mathrm{pm}$ intervals (and relaxed at each step) to locate the highest energy structure along the forming bond. The transition states for the Minisci reaction were fully optimized without any constrains.

Results and Discussion: To rationalize the more limited substrate scope of the Minisci reaction compared to the Giese reaction, we studied the addition of tert-butyl radical on methyl acrylate 
and protonated lepidine as model reactions for Giese and Minisci reactions, respectively. The energies and free energies for the reactions, as well as the transition state structures are shown in Table S4.

The transition state of the Minisci reaction is substantially later than that of the Giese reaction: The distance between the two sigma-bond forming carbons is $2.45 \AA$ in the Giese reaction and $2.14 \AA$ in the Minisci reaction. Both reactions are energetically barrierless (negative activation energies, see $\Delta \mathrm{E}^{\neq}$in Table $\left.\mathrm{S} 4\right)$, but destabilized by entropy: The activation free energies $\left(\Delta \mathrm{G}^{\neq}\right)$of both reactions are approximately $10 \mathrm{kcal} / \mathrm{mol}$ relative to isolated reactants. While the addition reactions are kinetically similar, the reaction free energies and their predicted reversibility differ significantly: The Giese reaction is exergonic by $-9.3 \mathrm{kcal} / \mathrm{mol}$ and thus considered irreversible, while the Minisci reaction is computed to be endergonic by $4.8 \mathrm{kcal} / \mathrm{mol}$. This implies that the latter is possibly reversible depending on the rate of the subsequent hydrogen atom abstraction.

Table S4. Activation (free) energies, reaction (free) energies and transition state structures for Minisci and Giese reactions. All energies are relative to isolated reactants.

\begin{tabular}{|c|c|c|}
\hline Reaction & Minisci & Giese \\
\hline Transition State & & \\
\hline$\Delta \mathbf{E}^{\neq} / \Delta \mathbf{G}^{\neq}(\mathrm{kcal} / \mathrm{mol})$ & $-3.8 / 10.2$ & $-3.4 / 10.5$ \\
\hline$\Delta E / \Delta G(\mathrm{kcal} / \mathrm{mol})$ & $-10.9 / 4.8$ & $-25.9 /-9.3$ \\
\hline
\end{tabular}

The computed activation free energy of $10.2 \mathrm{kcal} / \mathrm{mol}$ is similar to what reported by Fadeyi and coworkers for reaction between lepidine and cyclohexyl radical $(11.4 \mathrm{kcal} / \mathrm{mol}) .{ }^{12}$ The Minisci reaction with cyclohexyl radical, however, was predicted to be exergonic by $6 \mathrm{kcal} / \mathrm{mol}$. We consider this to originate from different stabilities of tert-butyl and cyclohexyl radicals, e.g., our 
computed reaction free energies for Minisci with isopropyl and ethyl radicals are 1.2 and -1.5 $\mathrm{kcal} / \mathrm{mol}$, respectively, see Table S5.

Table S5. Energetics and transition states for Minisci reaction with ethyl and isopropyl radicals.

\begin{tabular}{ccc}
\hline Radical & Et & $i-\operatorname{Pr}$ \\
\hline$\Delta \mathbf{E} / \Delta \mathbf{G}(\mathbf{k c a l} / \mathbf{m o l})$ & $-17.2 /-1.5$ & $-14.6 / 1.2$ \\
\hline
\end{tabular}

Cartesian coordinates: Cartesian coordinates of all optimized structures are provided as a separate text-file. 


\section{Excited State Kinetic Quenching Studies}

The triplet MLCT quenching experiments of $\operatorname{Ir}\left[\mathrm{dF}\left(\mathrm{CF}_{3}\right) \text { ppy }\right]_{2}(\mathrm{dtbbpy}) \mathrm{PF}_{6}$ were performed using the third harmonic of the Nd-YAG laser (Surelite, Continuum, $355 \mathrm{~nm}$ ) and $1 \times 1 \mathrm{~cm}$ quartz cuvettes. The emission from the sample has been collected at 90 degrees geometry to the excitation and coupled into the monochromator (Acton, Princeton Instruments) equipped with photomultiplier tube (Hamamatsu). Data collection has been performed using laboratory-written program based on LabView code. Samples of $\operatorname{Ir}\left[\mathrm{dF}\left(\mathrm{CF}_{3}\right) \text { ppy }\right]_{2}(\mathrm{dtbbpy}) \mathrm{PF}_{6}$ were prepared in solutions of DMSO with a total volume of $3 \mathrm{~mL}$ and an absorbance of $\sim 0.1$ at $355 \mathrm{~nm}$. The samples were degassed with Ar for 15 min prior to use. The solutions of Li methylcyclohexyl oxalate, lepidine, and $\left(\mathrm{NH}_{4}\right)_{2} \mathrm{~S}_{2} \mathrm{O}_{8}$ used for the quenching studies were also prepared in DMSO.

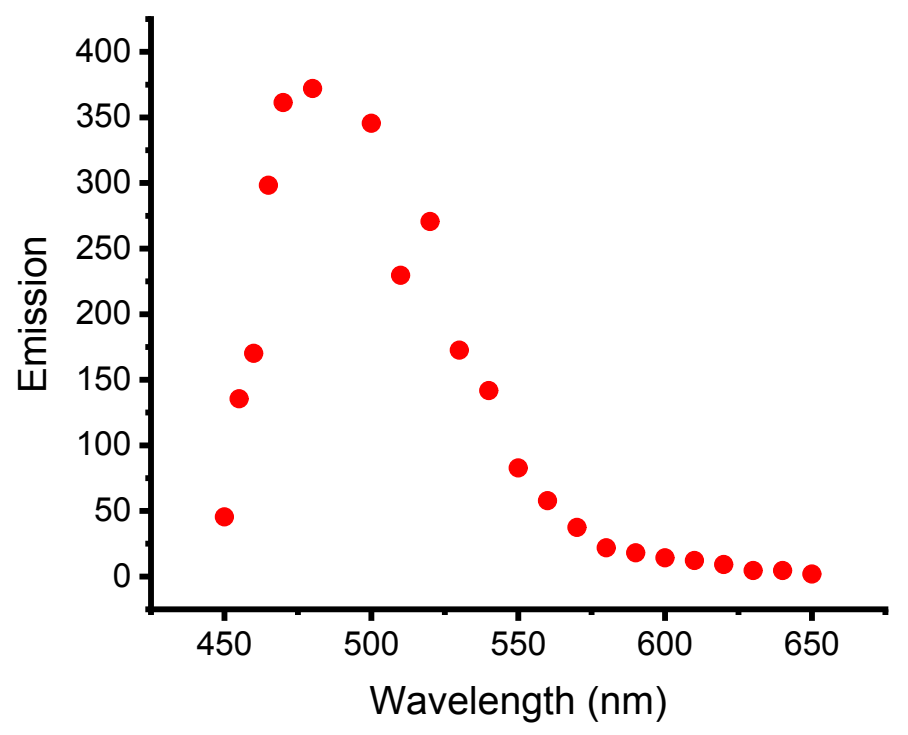

Figure S2. Emission spectrum of $\operatorname{Ir}\left[\mathrm{dF}\left(\mathrm{CF}_{3}\right) \text { ppy }\right]_{2}(\mathrm{dtbbpy}) \mathrm{PF}_{6}$ using $355 \mathrm{~nm}$ excitation.

Based on the recorded emission spectrum of $\operatorname{Ir}\left[\mathrm{dF}\left(\mathrm{CF}_{3}\right) \text { ppy }\right]_{2}(\mathrm{dtbbpy}) \mathrm{PF}_{6}$ displayed in Figure $\mathrm{S} 2$, $475 \mathrm{~nm}$ was chosen as the monitoring wavelength for the kinetic quenching studies. A typical 


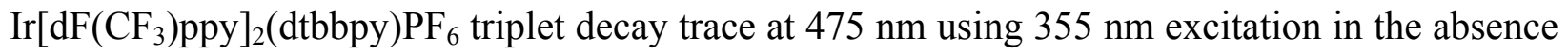
of a quencher is displayed in Figure S3.

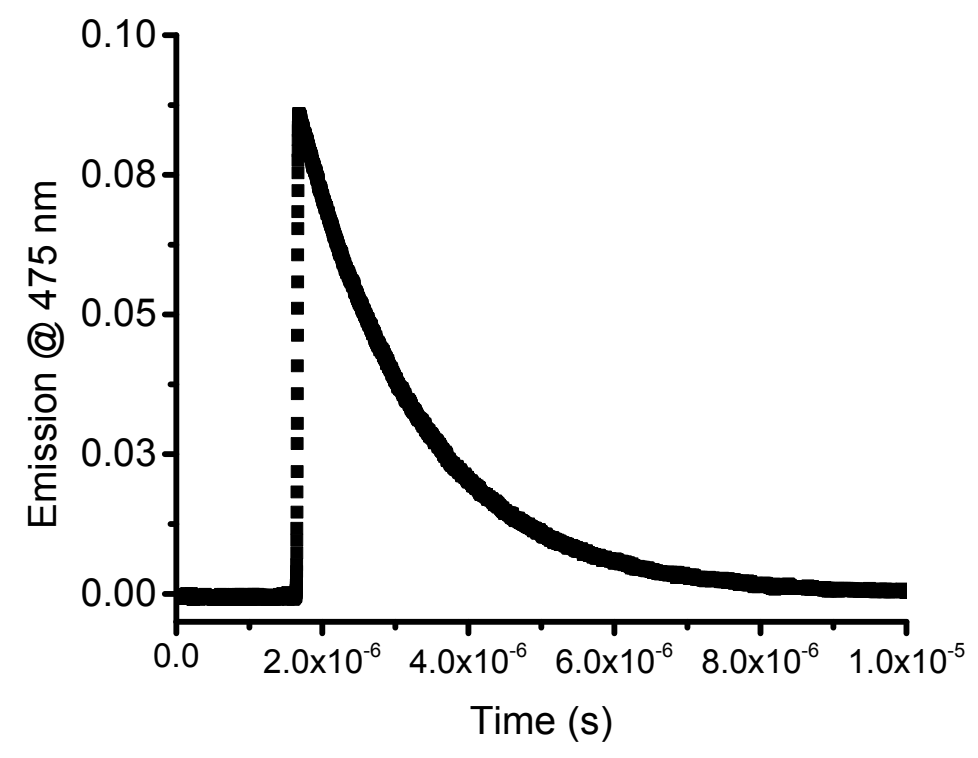

Figure S3. Raw data for the decay of the triplet emission of $\operatorname{Ir}\left[\mathrm{dF}\left(\mathrm{CF}_{3}\right) \mathrm{ppy}\right]_{2}(\mathrm{dtbbpy}) \mathrm{PF}_{6}$ in the absence of quencher in DMSO monitoring at $475 \mathrm{~nm}$.

Using OriginLab software, the decay traces were fit using the "Exponential" function to obtain $\mathrm{k}_{\mathrm{obs}}$. An example of the typical data output from OriginLab is displayed in Figure S4, using the fitting of the decay of $\operatorname{Ir}\left[\mathrm{dF}\left(\mathrm{CF}_{3}\right) \text { ppy }\right]_{2}(\mathrm{dtbbpy}) \mathrm{PF}_{6}$ in the absence of a quencher. 


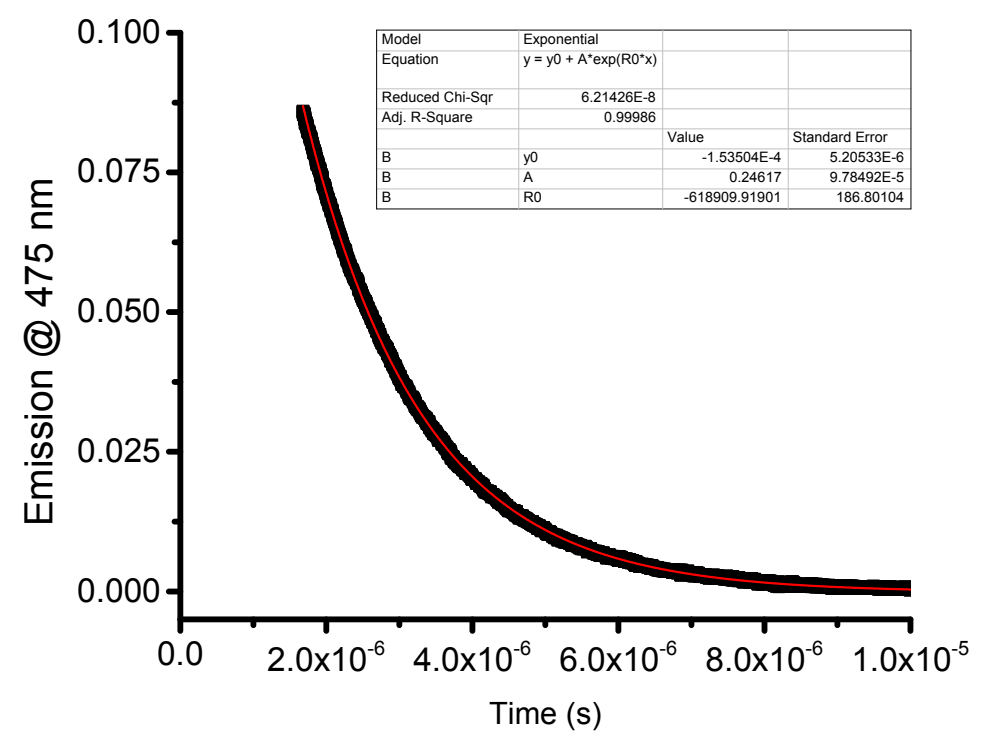

Figure S4. Analysis of the decay of $\operatorname{Ir}\left[\mathrm{dF}\left(\mathrm{CF}_{3}\right) \text { ppy }\right]_{2}(\mathrm{dtbbpy}) \mathrm{PF}_{6}$ in the absence of a quencher monitored at $475 \mathrm{~nm}$ in DMSO.

$$
\begin{aligned}
& {[\text { Quencher }]=[\text { Quencher }]_{0} e^{-k_{o b s} t}} \\
& \tau=\frac{\ln (2)}{k_{o b s}}
\end{aligned}
$$

From the data displayed in Figure $\mathrm{S} 4$, the lifetime of $\operatorname{Ir}\left[\mathrm{dF}\left(\mathrm{CF}_{3}\right) \mathrm{ppy}\right]_{2}(\mathrm{dtbbpy}) \mathrm{PF}_{6}$ in DMSO in the absence of a quencher $\left(\tau_{0}\right)$ was calculated to be $1.1 \mu$ s using equations (1) and (2). To obtain the bimolecular quenching constants $\left(\mathrm{k}_{\mathrm{q}}\right)$ of $\operatorname{Ir}\left[\mathrm{dF}\left(\mathrm{CF}_{3}\right) \text { ppy }\right]_{2}(\mathrm{dtbbpy}) \mathrm{PF}_{6}$ with Li methylcyclohexyl oxalate, lepidine, and $\left(\mathrm{NH}_{4}\right)_{2} \mathrm{~S}_{2} \mathrm{O}_{8}$, the decay of $\operatorname{Ir}\left[\mathrm{dF}\left(\mathrm{CF}_{3}\right) \text { ppy }\right]_{2}(\mathrm{dtbbpy}) \mathrm{PF}_{6}$ at $475 \mathrm{~nm}$ was monitored upon the addition of increasing concentrations of the quencher. Using pseudo-first order kinetic analysis, a plot of $\mathrm{k}_{\mathrm{obs}} \mathrm{vs}$. [Quencher] gives slope $=\mathrm{k}_{\mathrm{q}}$. 


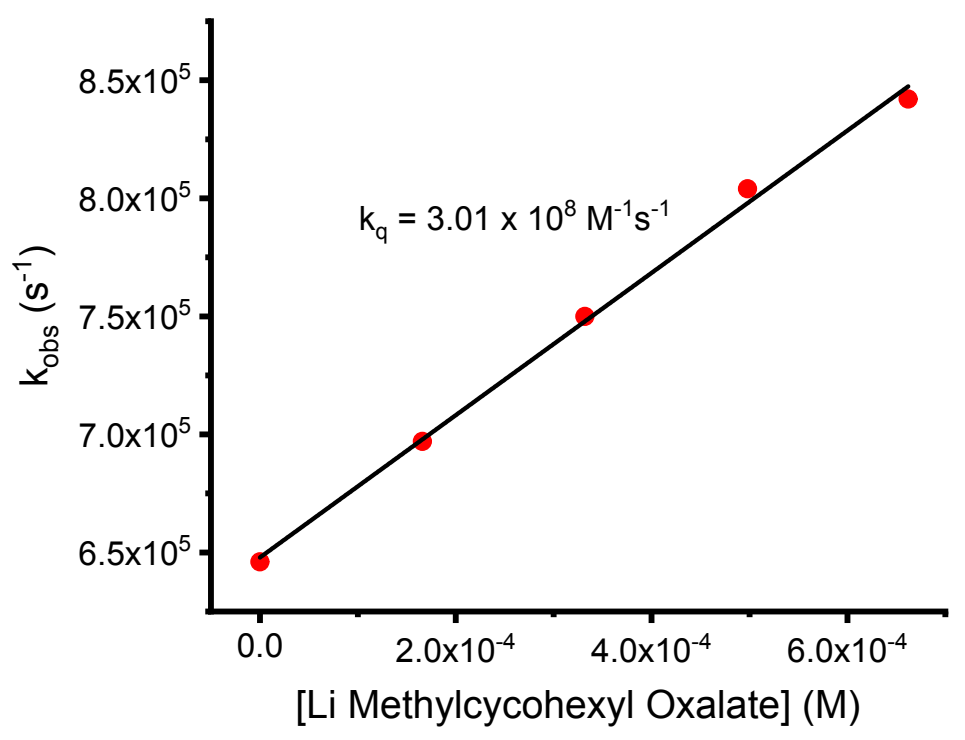

Figure S5. Representative kinetic plot for the quenching of $* \operatorname{Ir}\left[\mathrm{dF}\left(\mathrm{CF}_{3}\right) \mathrm{ppy}\right]_{2}(\mathrm{dtbbpy}) \mathrm{PF}_{6}$ by $\mathrm{Li}$ methylcyclohexyl oxalate.

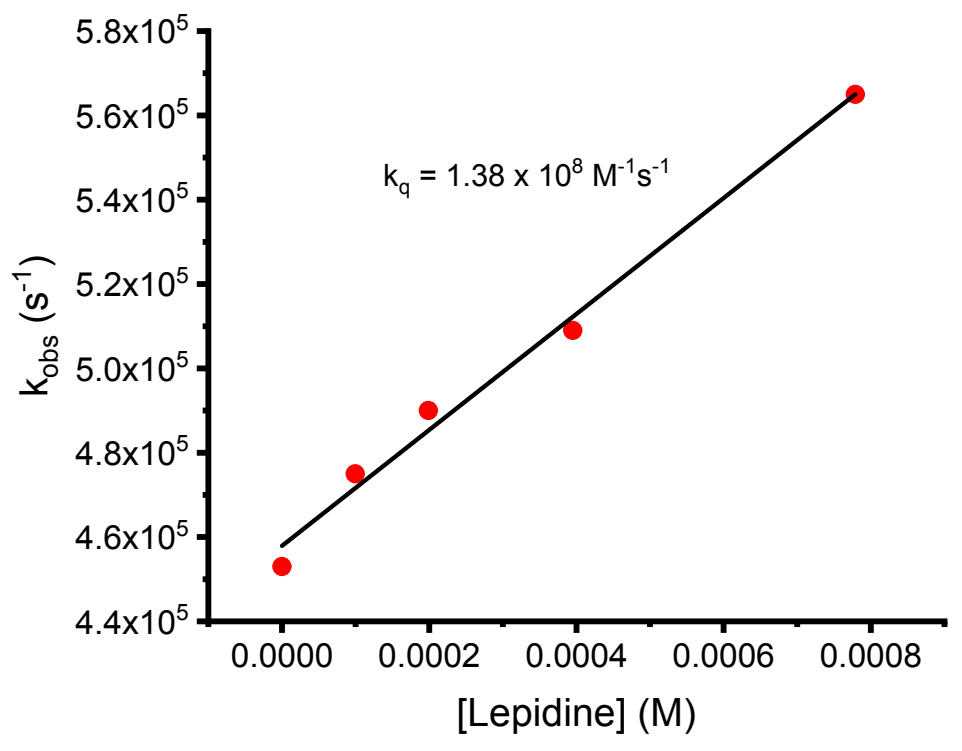

Figure S6. Representative kinetic plot for the quenching of $* \operatorname{Ir}\left[\mathrm{dF}\left(\mathrm{CF}_{3}\right) \mathrm{ppy}\right]_{2}(\mathrm{dtbbpy}) \mathrm{PF}_{6}$ by lepidine. 


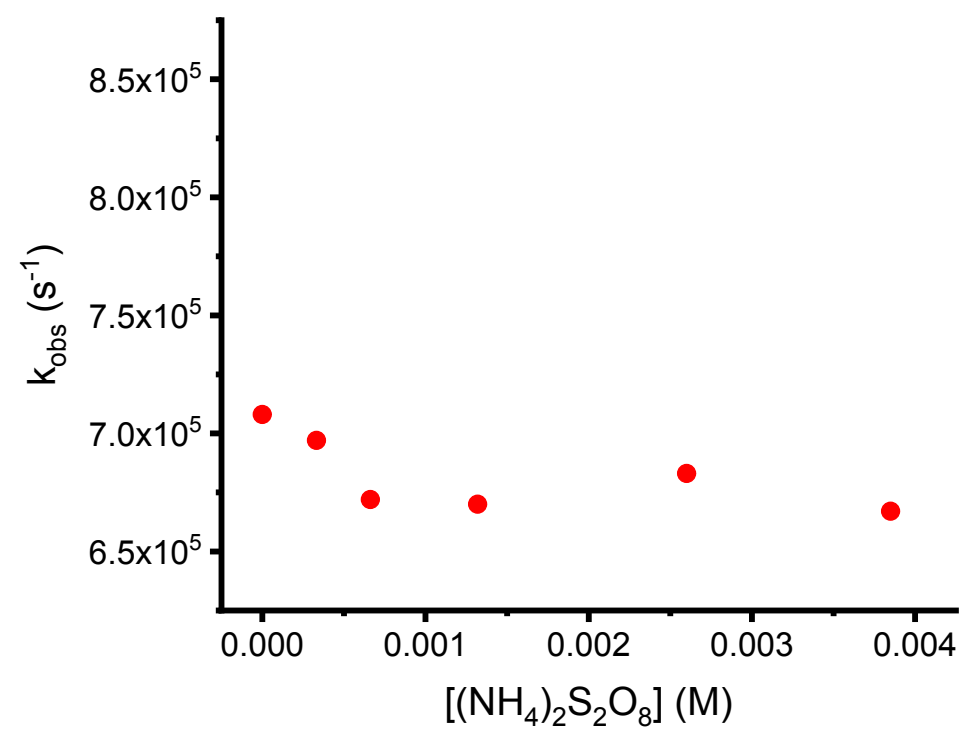

Figure S7. Representative kinetic plot for the quenching of $* \operatorname{Ir}\left[\mathrm{dF}\left(\mathrm{CF}_{3}\right) \text { ppy }\right]_{2}(\mathrm{dtbbpy}) \mathrm{PF}_{6}$ by $\left(\mathrm{NH}_{4}\right)_{2} \mathrm{~S}_{2} \mathrm{O}_{8}$.

Table S6. Bimolecular rate constants for the quenching of $* \operatorname{Ir}\left[\mathrm{dF}\left(\mathrm{CF}_{3}\right) \text { ppy }\right]_{2}(\mathrm{dtbbpy}) \mathrm{PF}_{6}$ by the individual reaction components of the Minisci reaction.

\begin{tabular}{cccc}
\multicolumn{4}{c}{$\mathbf{k}_{\mathbf{q}}\left(\mathbf{M}^{-1} \mathbf{s}^{-1}\right)$} \\
\hline Quencher & Li Methylcyclohexyl Oxalate $\left(\mathrm{k}_{1}\right)$ & Lepidine $\left(\mathrm{k}_{2}\right)$ & $\left(\mathrm{NH}_{4}\right)_{2} \mathrm{~S}_{2} \mathrm{O}_{8}\left(\mathrm{k}_{3}\right)$ \\
\hline Trial 1 & $3.07 \times 10^{8}$ & $1.29 \times 10^{8}$ & N/A \\
Trial 2 & $3.01 \times 10^{8}$ & $1.21 \times 10^{8}$ & N/A \\
Trial 3 & $3.79 \times 10^{8}$ & $1.38 \times 10^{8}$ & N/A \\
\hline Average & $3.29 \pm 0.43 \times 10^{8}$ & $1.29 \pm 0.09 \times 10^{8}$ & N/A
\end{tabular}




\section{J. Determination of the Quantum Yield for the Photoredox-Catalyzed Minisci Reaction}

In order to determine the quantum yield for the photoredox-catalyzed Minisci reaction, we employed an actinometer based on $\mathrm{Ru}(\mathrm{bpy})_{3} \mathrm{Cl}_{2}$ previously reported by Scaiano and coworkers. ${ }^{13}$ In order to facilitate spectral matching between the $\mathrm{Ru}(\mathrm{bpy})_{3} \mathrm{Cl}_{2}$ actinometer and the $\operatorname{Ir}\left[\mathrm{dF}\left(\mathrm{CF}_{3}\right) \mathrm{ppy}\right]_{2}(\mathrm{dtbbpy}) \mathrm{PF}_{6}$ photocatalyst, a cutoff filter from Thor Labs $(420 \pm 10 \mathrm{~nm})$ was placed in front of one of our Kessil LED lamps. As shown in Figure S8, $\left[\mathrm{Ru}(\mathrm{bpy})_{3} \mathrm{Cl}_{2}\right]=0.5 \mathrm{mM}$ was employed to match the absorption of our Ir photocatalyst over the area of emission for our Kessil LED lamp equipped with a $420 \pm 10$ nm cutoff filter.

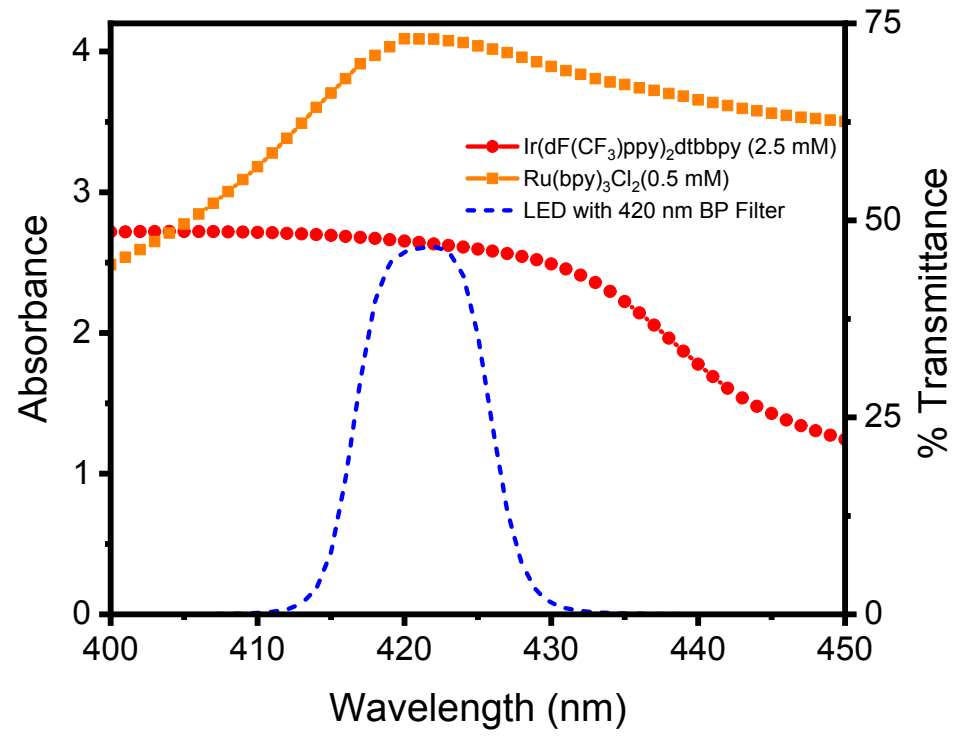

Figure S8. Absorption spectra of $\operatorname{Ir}\left[\mathrm{dF}\left(\mathrm{CF}_{3}\right) \mathrm{ppy}\right]_{2}(\mathrm{dtbbpy}) \mathrm{PF}_{6}$ at the same concentration employed in the photoredox-catalyzed Minisci reaction $\left(2.5 \mathrm{mM}\right.$, red) and $\mathrm{Ru}(\mathrm{bpy})_{3} \mathrm{Cl}_{2}(0.5 \mathrm{mM}$, orange) overlaid with the emission profile of the Thor Labs $420 \pm 10 \mathrm{~nm}$ cutoff filter (blue).

A $100 \mathrm{~mL}$ stock solution of the actinometer was made with $0.5 \mathrm{mM} \mathrm{Ru}(\mathrm{bpy})_{3} \mathrm{Cl}_{2}(18.7 \mathrm{mg})$ and 0.10 mM 9,10-diphenylanthracene (DPA, $3.3 \mathrm{mg}$ ) in MeCN. $3 \mathrm{~mL}$ of the stock solution was added to a quartz cuvette, and a UV-Vis spectrum was recorded from $450-350 \mathrm{~nm}\left(\mathrm{~A}_{\text {initial }}\right)$. The cuvette 
was then placed in front of the Kessil LED lamp equipped with a Thor Labs $420 \pm 10 \mathrm{~nm}$ cutoff filter and was irradiated for 120 seconds. Following irradiation, the absorption spectrum was recorded again from $450-350 \mathrm{~nm}\left(\mathrm{~A}_{\text {final }}\right)$. This was repeated in triplicate, and by employing equations (3) and (4):

$$
\begin{aligned}
& \text { moles of DPA consumed }=\frac{\left(A_{\text {initial }}-A_{\text {final }}\right)}{\left(\varepsilon_{372 \mathrm{~nm}}\right)(l)}(V) \\
& \frac{N h v}{t}=I_{0}=\frac{\text { moles of DPA consumed }}{(\Phi)(t)}
\end{aligned}
$$

where $A_{\text {initial }}$ and $A_{\text {final }}$ are the absorbances at $372 \mathrm{~nm}$ before and after irradiation, $\varepsilon_{372 \mathrm{~nm}}$ is the extinction coefficient of DPA at $372 \mathrm{~nm}\left(11,100 \mathrm{M}^{-1} \mathrm{~cm}^{-1}\right), 1$ is the path length and $\Phi$ is the quantum yield of the actinometer $(0.019)^{13}$, it was calculated that $I_{0}$ for the $460 \mathrm{~nm}$ LED set up employed was $8.92 \pm 0.51 \times 10^{-9} \mathrm{~mol} \mathrm{hv} \mathrm{s}{ }^{-1}$.

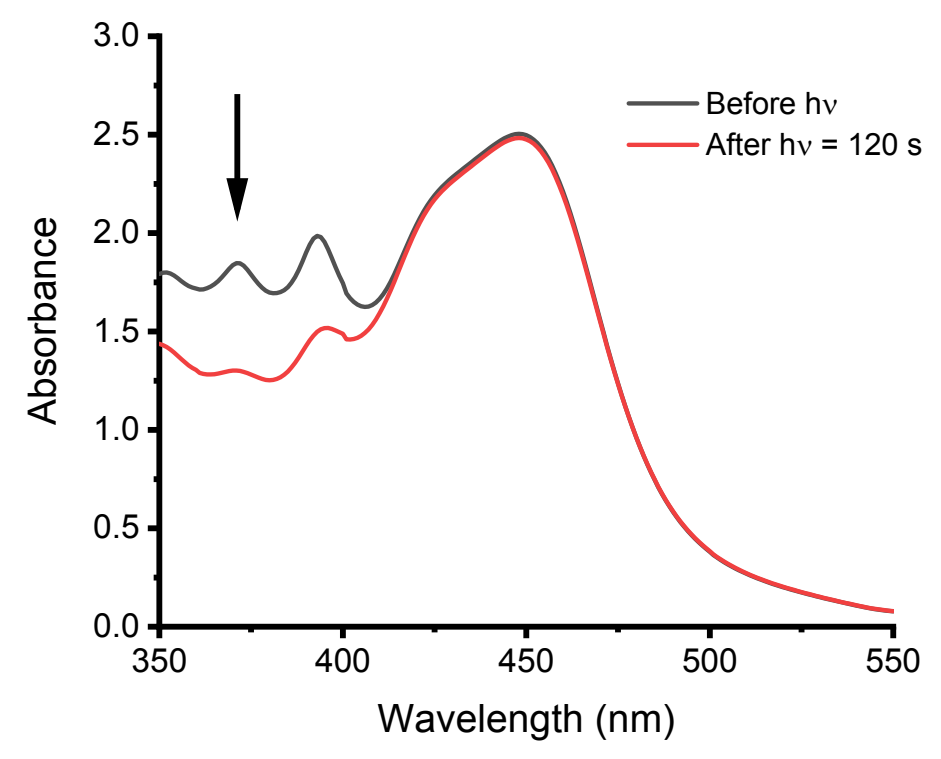

Figure S9. Typical example of raw data for the $\mathrm{Ru}(\mathrm{bpy})_{3} \mathrm{Cl}_{2}$ actinometry experiment performed to calculate the number of photons arriving at the sample in a given period of time using a Kessil LED lamp equipped with a $420 \pm 10$ nm cutoff filter. 
In order to calculate the quantum yield of the photoredox-catalyzed Minisci reaction, the initial reaction rate was calculated. A $3 \mathrm{~mL}$ quartz cuvette equipped with a magnetic stir bar was charged with cesium tert-butyloxalate $\left(167 \mathrm{mg}, 0.6 \mathrm{mmol}, 1.2\right.$ equiv), $\operatorname{Ir}\left[\mathrm{dF}\left(\mathrm{CF}_{3}\right) \mathrm{ppy}\right]_{2}(\mathrm{dtbbpy}) \mathrm{PF}_{6}(2.8$ mg, $0.0025 \mathrm{mmol}, 0.5 \mathrm{~mol} \%)$, and $\left(\mathrm{NH}_{4}\right)_{2} \mathrm{~S}_{2} \mathrm{O}_{8}(171 \mathrm{mg}, 0.75 \mathrm{mmol}, 1.5$ equiv). Dry DMSO (1 $\mathrm{mL}, 0.5 \mathrm{M}$ ) and lepidine ( $66 \mathrm{~mL}, 0.5 \mathrm{mmol}, 1.0$ equiv) were then added, and the reaction mixture was degassed by sparging with argon for $10 \mathrm{~min}$. The reaction mixture was then sonicated and irradiated with the same Kessil LED lamp equipped with a Thor Labs $420 \pm 10 \mathrm{~nm}$ cutoff filter for $10 \mathrm{~min}$. The reaction temperature was maintained at $30^{\circ} \mathrm{C}$ by blowing air into the photochemistry setup. The reaction mixture was transferred into a separatory funnel and diluted with $25 \mathrm{~mL}$ of $\mathrm{EtO}_{2}$ and quenched with $25 \mathrm{~mL}$ of saturated $\mathrm{NaHCO}_{3(\mathrm{aq})}$. The $\mathrm{EtO}_{2}$ phase was washed with brine ( $2 \times 25 \mathrm{~mL})$, and the combined aqueous phases were extracted with $\mathrm{EtO}_{2}(2 \times 25 \mathrm{~mL})$. The combined organic phases were dried with $\mathrm{MgSO}_{4}$ and concentrated. The yield of the reaction was calculated by ${ }^{1} \mathrm{H}$ NMR using 1,2-dibromo-3,4-methylenedioxybenzene as an external standard. The yield after $10 \mathrm{~min}(600 \mathrm{~s}$ ) of irradiation was calculated to be $13.2 \%$, equivalent to $0.066 \mathrm{mmol}$ of product produced. Using equation (5),

$$
\Phi=\frac{(\text { moles of product produced })}{t \times F} \times\left(\frac{N h v}{t}\right)^{-1}
$$

where $t=600 \mathrm{~s}, F=1.0$, and $\mathrm{Nh} v / \mathrm{t}=8.92 \times 10^{-9} \mathrm{~mol} \mathrm{hv} \mathrm{s}^{-1}$, the quantum yield $(\Phi)$ for the photoredox-catalyzed Minisci reaction was calculated to be 12, indicating that chain propagation is part of the underlying mechanism of the transformation. 


\section{K. Preparation of Starting Materials}

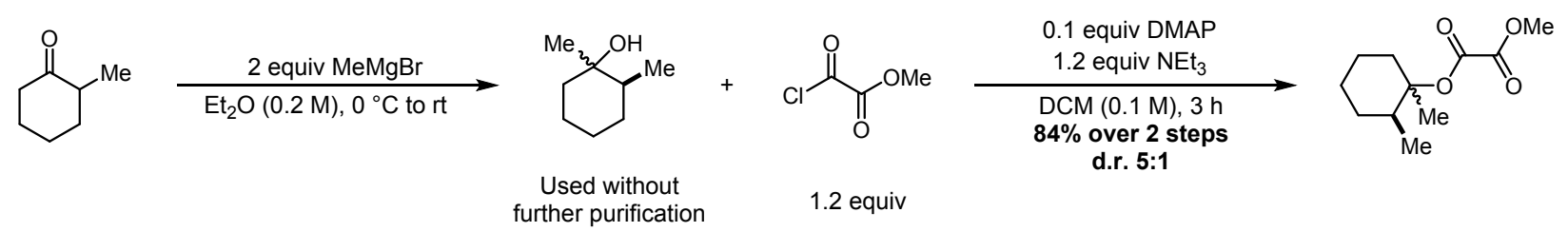

1,2-Dimethylcyclohexyl methyl oxalate: A solution of methylmagnesium bromide (2.9 $\mathrm{M}$ in $\mathrm{Et}_{2} \mathrm{O} ; 3.4 \mathrm{~mL}, 10.0 \mathrm{mmol}, 2$ equiv) was added dropwise to 2 -methylcyclohexanone $(610 \mu \mathrm{L}, 5.0$ mmol, 1.0 equiv $)$ in $\mathrm{Et}_{2} \mathrm{O}(25 \mathrm{~mL})$ at $0{ }^{\circ} \mathrm{C}$. The resulting solution was warmed to room temperature and stirred overnight. Upon completion, the reaction was quenched with a saturated aqueous $\mathrm{NH}_{4} \mathrm{Cl}$ solution $(20 \mathrm{~mL})$. The layers were separated, and the aqueous phase was extracted with $\mathrm{Et}_{2} \mathrm{O}(2 \times 20 \mathrm{~mL})$. The combined $\mathrm{Et}_{2} \mathrm{O}$ phases were washed with brine $(20 \mathrm{~mL})$, dried over $\mathrm{MgSO}_{4}$ and concentrated to yield 1,2-dimethylcyclohexanol ${ }^{14}$ as a mixture of stereoisomers, which was used in the next step without further purification.

Methyl chlorooxoacetate $(552 \mu \mathrm{L}, 6.0 \mathrm{mmol}, 1.2$ equiv) was added to a solution of crude $1,2-$ dimethylcyclohexanol, DMAP (61 mg, $0.5 \mathrm{mmol}, 0.1$ equiv) and $\mathrm{NEt}_{3}(840 \mu \mathrm{L}, 6.0 \mathrm{mmol}, 1.2$ equiv) in DCM (50 mL) and the resulting solution was stirred at room temperature for 3 hours. Upon completion, the reaction was quenched with a saturated aqueous $\mathrm{NH}_{4} \mathrm{Cl}$ solution $(20 \mathrm{~mL})$. The layers were separated, and the aqueous phase was extracted with DCM (2 x $20 \mathrm{~mL})$. The combined organic phases were washed with saturated aqueous $\mathrm{NaHCO}_{3}$ solution $(20 \mathrm{~mL})$ and brine $(20 \mathrm{~mL})$, dried over $\mathrm{MgSO}_{4}$ and concentrated. The crude material was purified by flash column chromatography (Hex $\rightarrow$ 85:15 Hex:EtOAc) to give the title compound as a clear oil in 5:1 dr (902 mg, 84\% yield over two steps).

${ }^{1}$ H NMR: $\left(600 \mathrm{MHz}, \mathrm{CDCl}_{3}\right) \delta 3.87$ (s, 3H), 2.72-2.64 (m, 1H), 1.71-1.64 (m, 1H), $1.57(\mathrm{~s}, 3 \mathrm{H})$, 1.54-1.42 (m, 4H), 1.37-1.24 (m, 3H), 0.99 (d, $J=6.38 \mathrm{~Hz}, 3 \mathrm{H}) .{ }^{13} \mathbf{C}$ NMR: $\left(151 \mathrm{MHz}, \mathrm{CDCl}_{3}\right)$ 
$\delta 159.2,157.0,88.1,53.3,41.9,34.6,30.2,25.4,24.0,21.9,15.3$. Rf: 0.47 (4:1 Hex:EtOAc).

HRMS (m/z): Calculated for $\mathrm{C}_{11} \mathrm{H}_{18} \mathrm{O}_{4}\left([\mathrm{M}+\mathrm{Na}]^{+}\right)$: 237.1103, found: 237.1103. IR (neat, $\left.\mathbf{c m}^{-1}\right)$ : 2932, 2860, 2360, 1765, 1740, 1457, 1327, 1205, 1148, 1093, 787.
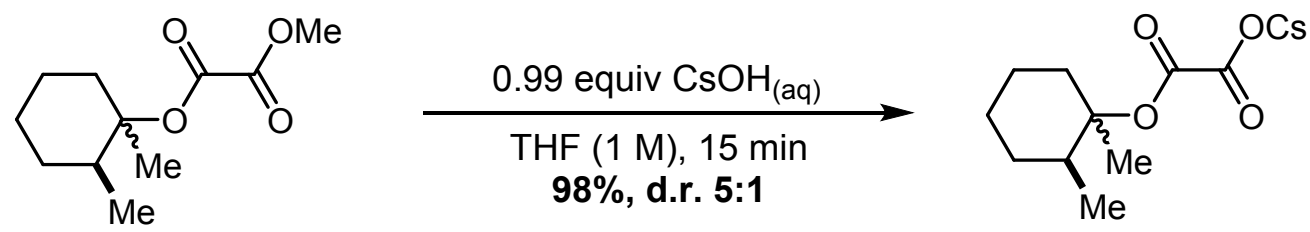

Cesium 2-((1,2-dimethylcyclohexyl)oxy)-2-oxoacetate: A round bottom flask was charged with 1,2-dimethylcyclohexyl methyl oxalate $(878 \mathrm{mg}, 4.1 \mathrm{mmol}, 1.0$ equiv) followed by the addition of THF (5 mL). To this solution, $1 \mathrm{~N}$ aq. $\mathrm{CsOH}(4.06 \mathrm{~mL}, 4.06 \mathrm{mmol}, 0.99$ equiv) was added dropwise. The mixture was stirred vigorously for $15 \mathrm{~min}$ at room temperature, then concentrated under reduced pressure to give the title compound as a colorless solid in 5:1 $\mathrm{dr}(1.33 \mathrm{~g}, 98 \%$ yield $)$.

${ }^{1}$ H NMR: $\left(600 \mathrm{MHz}, \mathrm{DMSO}_{\mathrm{d}}\right) \delta$ 2.55-2.50 (m, 1H), 1.62-1.52 (m, 1H), $1.40(\mathrm{~s}, 3 \mathrm{H}), 1.40-1.32$ $(\mathrm{m}, 5 \mathrm{H}), 1.28-1.11(\mathrm{~m}, 3 \mathrm{H}), 0.87(\mathrm{~d}, J=6.08 \mathrm{~Hz}, 3 \mathrm{H}) .{ }^{13} \mathrm{C}$ NMR: (151 MHz, DMSO-d $\left.\mathrm{d}_{6}\right)$ $\delta$ 167.7, 163.6, 80.5, 41.2, 34.2, 29.7, 25.0, 24.0, 21.3, 15.1. HRMS $(\mathbf{m} / \mathbf{z})$ : Calculated for $\mathrm{C}_{10} \mathrm{H}_{15} \mathrm{CsO}_{4}\left([\mathrm{M}-\mathrm{Cs}]^{-}\right): 199.0970$, found: 199.0972. IR (neat, $\mathbf{c m}^{-1}$ ): 2931, 2854, 1705, 1643, $1616,1210,1153,777$.
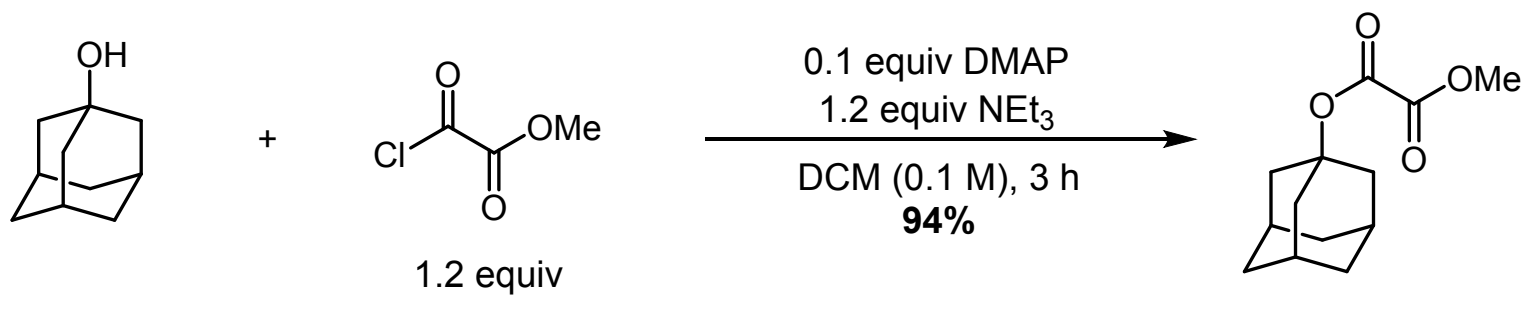

Adamantan-1-yl methyl oxalate: Methyl chlorooxoacetate $(717 \mu \mathrm{L}, 7.8 \mathrm{mmol}, 1.2$ equiv) was added to a solution of 1-adamantanol (1.0 g, $6.5 \mathrm{mmol}, 1.0$ equiv), DMAP (79 mg, $0.65 \mathrm{mmol}, 0.1$ 
equiv) and $\mathrm{NEt}_{3}(1.61 \mathrm{~mL}, 6.0 \mathrm{mmol}, 1.2$ equiv) in $\mathrm{DCM}(65 \mathrm{~mL})$ and the resulting solution was stirred at room temperature for 3 hours. Upon completion, the reaction was quenched with a saturated aqueous $\mathrm{NH}_{4} \mathrm{Cl}$ solution $(40 \mathrm{~mL}$ ). The layers were separated, and the aqueous phase was extracted with DCM $(2 \times 40 \mathrm{~mL})$. The combined organic phases were washed with saturated aqueous $\mathrm{NaHCO}_{3}$ solution $(40 \mathrm{~mL})$ and brine $(40 \mathrm{~mL})$, dried over $\mathrm{MgSO}_{4}$ and concentrated. The crude material was purified by flash column chromatography (Hex $\rightarrow$ 9:1 Hex:EtOAc) to give the title compound as a colorless solid (1.46 mg, 94\% yield over two steps).

${ }^{1}$ H NMR: $\left(600 \mathrm{MHz}, \mathrm{CDCl}_{3}\right) \delta 3.86(\mathrm{~s}, 3 \mathrm{H}), 2.23-2.20(\mathrm{~m}, 3 \mathrm{H}), 2.20-2.18(\mathrm{~m}, 6 \mathrm{H}), 1.72-1.65(\mathrm{~m}$, 6H). ${ }^{13}$ C NMR: $\left(151 \mathrm{MHz}, \mathrm{CDCl}_{3}\right) \delta 159.2,156.5,85.3,53.4,41.1,36.1,31.1$. Rf: 0.44 (4:1 Hex:EtOAc). HRMS (m/z): Calculated for $\mathrm{C}_{13} \mathrm{H}_{18} \mathrm{O}_{4}\left([\mathrm{M}+\mathrm{Na}]^{+}\right)$: 261.1103, found: 261.1099. IR (neat, $\left.\mathbf{c m}^{-1}\right):$ 2912, 2853, 1764, 1736, 1331, 1199, 1159, 1045, 963, 783 .
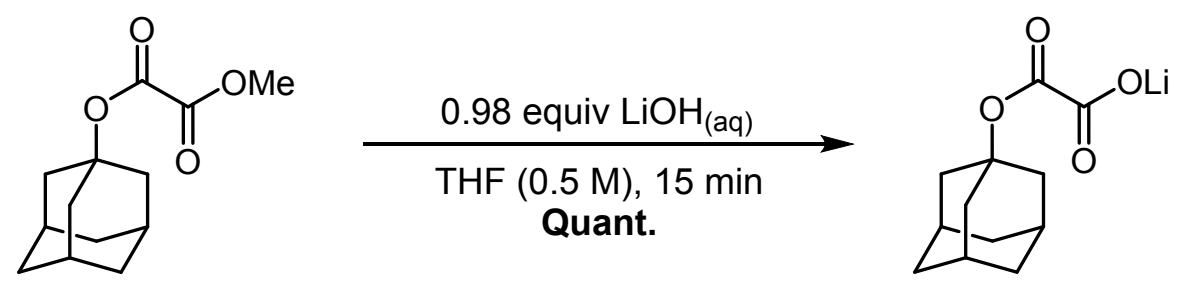

Lithium 2-((adamantan-1-yl)oxy)-2-oxoacetate: A round bottom flask was charged with adamantan-1-yl methyl oxalate (1.437 g, $6.0 \mathrm{mmol}, 1.0$ equiv) followed by the addition of THF (12 mL). To this solution, $0.5 \mathrm{~N}$ aq. $\mathrm{LiOH}(11.8 \mathrm{~mL}, 5.91 \mathrm{mmol}, 0.98$ equiv) was added dropwise. The mixture was stirred vigorously for $15 \mathrm{~min}$ at room temperature, then concentrated under reduced pressure to give the title compound as a colorless solid (1.39 g, quantitative yield).

${ }^{1}$ H NMR: $\left(600 \mathrm{MHz}\right.$, DMSO-d 6 ) $\delta$ 2.12-2.08 (m, 3H), 2.05-2.02 (m, 6H), 1.64-1.58 (m, 6H). ${ }^{13} \mathbf{C}$ NMR: (151 MHz, DMSO-d $\left.\mathrm{d}_{6}\right) \delta 166.9,163.2,78.3,41.0,35.7,30.2$. HRMS (m/z): Calculated for 
$\mathrm{C}_{12} \mathrm{H}_{15} \mathrm{LiO}_{4}\left([\mathrm{M}-\mathrm{Li}]^{-}\right):$223.0970, found: 223.0978. IR (neat, $\mathbf{c m}^{-1}$ ): 2908, 2950, 1710, 1660, $1248,1048,787$.

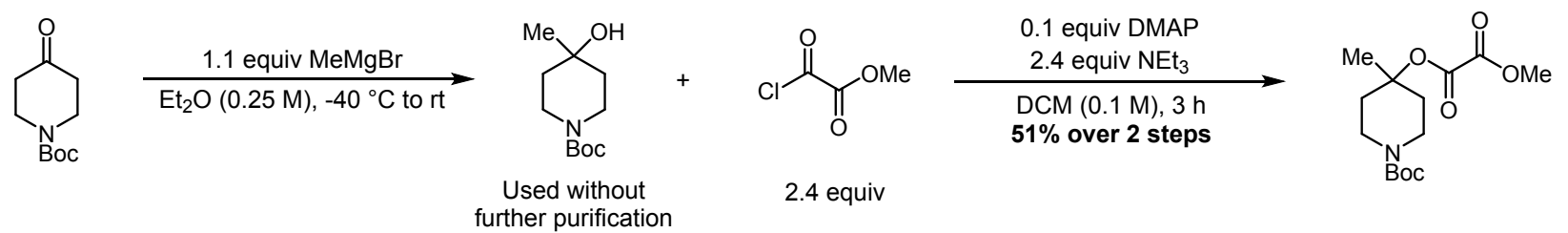

1-(tert-butoxycarbonyl)-4-methylpiperidin-4-yl methyl oxalate: A solution of methyl magnesium bromide (2.9 $\mathrm{M}$ in $\mathrm{Et}_{2} \mathrm{O} ; 1.9 \mathrm{~mL}, 5.5 \mathrm{mmol}, 1.1$ equiv) was added dropwise to 1Boc-4-piperidone $\left(1.0 \mathrm{~g}, 5.0 \mathrm{mmol}, 1.0\right.$ equiv) in $\mathrm{Et}_{2} \mathrm{O}(20 \mathrm{~mL})$ at $-40{ }^{\circ} \mathrm{C}$. The resulting solution was stirred at $-40{ }^{\circ} \mathrm{C}$ for $5 \mathrm{~min}$, allowed to warm to room temperature, and stirred for an additional 2 hours. Upon completion, the reaction was quenched with a saturated aqueous $\mathrm{NH}_{4} \mathrm{Cl}$ solution $(20 \mathrm{~mL})$ and diluted with $\mathrm{Et}_{2} \mathrm{O}(20 \mathrm{~mL})$. The layers were separated, and the aqueous phase was extracted with $\mathrm{Et}_{2} \mathrm{O}(2 \times 20 \mathrm{~mL})$. The combined $\mathrm{Et}_{2} \mathrm{O}$ phases were washed with brine $(20 \mathrm{~mL})$, dried over $\mathrm{MgSO}_{4}$ and concentrated to yield tert-butyl 4-hydroxy-4-methylpiperidine-1carboxylate ${ }^{15}$ which was used in the next step without further purification.

Methyl chlorooxoacetate (552 $\mu \mathrm{L}, 6.0 \mathrm{mmol}, 1.2$ equiv) was added to a solution of crude tert-butyl 4-hydroxy-4-methylpiperidine-1-carboxylate, DMAP (61 mg, $0.5 \mathrm{mmol}, 0.1$ equiv) and $\mathrm{NEt}_{3}(840$ $\mu \mathrm{L}, 6.0 \mathrm{mmol}, 1.2$ equiv) in $\mathrm{DCM}(50 \mathrm{~mL})$ and the resulting solution was stirred at room temperature for 2 hours. Additional methyl chlorooxoacetate $(552 \mu \mathrm{L}, 6.0 \mathrm{mmol}, 1.2$ equiv) and $\mathrm{NEt}_{3}(840 \mu \mathrm{L}, 6.0 \mathrm{mmol}, 1.2$ equiv) were added, and the reaction mixture was stirred for another hour. Upon completion, the reaction was quenched with a saturated aqueous $\mathrm{NH}_{4} \mathrm{Cl}$ solution $(20$ $\mathrm{mL})$. The layers were separated, and the aqueous phase was extracted with DCM $(2 \times 20 \mathrm{~mL})$. The combined organic phases were washed with saturated aqueous $\mathrm{NaHCO}_{3}$ solution $(20 \mathrm{~mL})$ and brine $(20 \mathrm{~mL})$, dried over $\mathrm{MgSO}_{4}$ and concentrated. The crude material was purified by flash 
column chromatography (Hex $\rightarrow$ 75:25 Hex:EtOAc) to give the title compound as a yellow oil (765 mg, 51\% yield over two steps).

${ }^{1}$ H NMR: (600 MHz, $\left.\mathrm{CDCl}_{3}\right) \delta 3.87$ (s, 3H), 3.86-3.71 (b.s, 2H), 3.15-3.05 (m, 2H), 2.28-2.22 (m, 2H), 1.65-1.59 (m, 5H), 1.45 (s, 9H). ${ }^{13}$ C NMR: (151 MHz, $\left.\mathrm{CDCl}_{3}\right) \delta$ 158.8, 156.7, 154.8, 84.1, 79.9, 53.5, 40.1, 39.3, 35.7, 28.6, 24.8. Rf: 0.29 (4:1 Hex:EtOAc). HRMS (m/z): Calculated for $\mathrm{C}_{14} \mathrm{H}_{23} \mathrm{NO}_{6}\left([\mathrm{M}+\mathrm{Na}]^{+}\right): 324.1423$, found: 324.1418. IR (neat, $\left.\mathbf{c m}^{-1}\right): 2976,1766,1690,1422$, $1365,1324,1261,1202,1136,966,789$.<smiles>COC(=O)C(=O)OC1(C)CCN(C(=O)OCc2ccccc2)CC1</smiles>
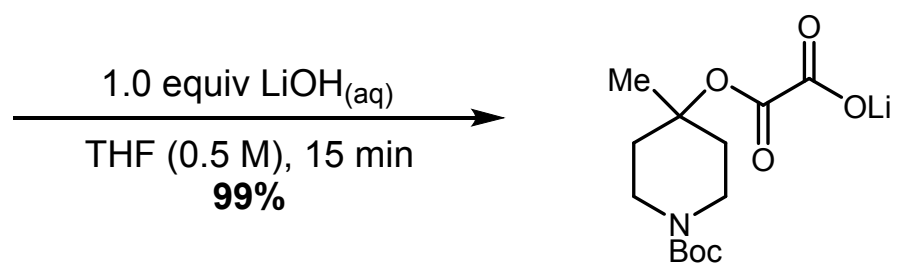

Lithium 2-((1-(tert-butoxycarbonyl)-4-methylpiperidin-4-yl)oxy)-2-oxoacetate: A round bottom flask was charged with 1-(tert-butoxycarbonyl)-4-methylpiperidin-4-yl methyl oxalate (710 mg, $2.36 \mathrm{mmol}, 1.0$ equiv) followed by the addition of THF ( $5 \mathrm{~mL}$ ). To this solution, $0.5 \mathrm{~N}$ aq. $\mathrm{LiOH}(4.72 \mathrm{~mL}, 2.36 \mathrm{mmol}, 1.0$ equiv) was added dropwise. The mixture was stirred vigorously for $15 \mathrm{~min}$ at room temperature, then concentrated under reduced pressure to give the title compound as an off-white solid (683 $\mathrm{mg}, 99 \%$ yield).

${ }^{1}$ H NMR: $\left(600 \mathrm{MHz}\right.$, DMSO-d $\left.{ }_{6}\right) \delta 3.61(\operatorname{app~d}, J=11.3 \mathrm{~Hz}, 1 \mathrm{H}), 2.03($ app d, $J=13.3 \mathrm{~Hz}, 1 \mathrm{H})$, 1.50-1.45 (m, 2H), 1.44 (s, 3H), 1.39 (s, 9H). ${ }^{13}$ C NMR: (151 MHz, DMSO-d 6 ) $\delta$ 167.1, 163.1, 154.0, 78.6, 77.4, 35.3, 28.12, 28.08, 24.7. HRMS (m/z): Calculated for $\mathrm{C}_{13} \mathrm{H}_{20} \mathrm{LiNO}_{4}\left([\mathrm{M}-\mathrm{Li}]^{-}\right)$: 286.1291, found: 286.1293. IR (neat, $\mathbf{c m}^{-1}$ ): 2975, 1660, 1422, 1366, 1231, 1142, 991, 907, 790. 


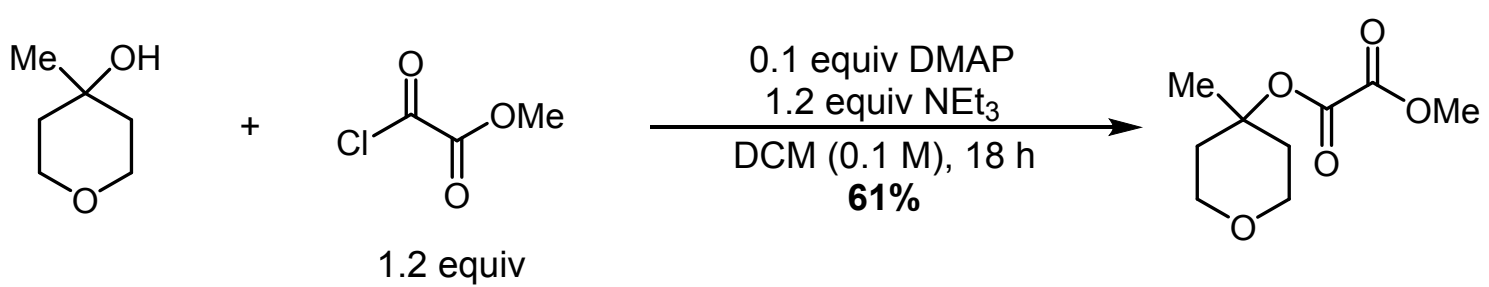

Methyl (4-methyltetrahydro-2H-pyran-4-yl) oxalate: Methyl chlorooxoacetate (485 $\mu \mathrm{L}, 5.27$ mmol, 1.2 equiv) was added to a solution of 4-methyltetrahydro- $2 H$-pyran-4-ol ${ }^{16}(510 \mathrm{mg}, 4.39$ mmol, 1.0 equiv), DMAP (54 mg, $0.44 \mathrm{mmol}, 0.1$ equiv) and $\mathrm{NEt}_{3}(1.1 \mathrm{~mL}, 5.27 \mathrm{mmol}, 1.2$ equiv) in DCM $(50 \mathrm{~mL})$ and the resulting solution was stirred at room temperature overnight. Upon completion, the reaction was quenched with a saturated aqueous $\mathrm{NH}_{4} \mathrm{Cl}$ solution $(25 \mathrm{~mL})$. The layers were separated, and the aqueous phase was extracted with DCM $(2 \times 25 \mathrm{~mL})$. The combined organic phases were washed with saturated aqueous $\mathrm{NaHCO}_{3}$ solution $(25 \mathrm{~mL})$ and brine $(25 \mathrm{~mL})$, dried over $\mathrm{MgSO}_{4}$ and concentrated. The crude material was purified by flash column chromatography (Hex $\rightarrow$ 4:1 Hex:EtOAc) to give the title compound as a pale yellow oil (544 mg, $61 \%)$

${ }^{1} \mathbf{H}$ NMR: $\left(600 \mathrm{MHz}, \mathrm{CDCl}_{3}\right) \delta 3.89(\mathrm{~s}, 3 \mathrm{H}), 3.77-3.72(\mathrm{~m}, 2 \mathrm{H}), 3.71-.366(\mathrm{~m}, 2 \mathrm{H}), 2.24-2.20(\mathrm{~m}$, 2H), 1.82-1.76 (m, 2H), $\left.1.63(\mathrm{~s}, 3 \mathrm{H}) .{ }^{13} \mathrm{C} \mathrm{NMR:} \mathrm{(151} \mathrm{MHz,} \mathrm{CDCl}_{3}\right) \delta 158.8,156.8,83.4,63.7$, 53.5, 36.6, 24.9. Rf: 0.21 (4:1 Hex:EtOAc). HRMS (m/z): Calculated for $\mathrm{C}_{9} \mathrm{H}_{14} \mathrm{O}_{5}\left([\mathrm{M}+\mathrm{Na}]^{+}\right)$: 225.0739, found: 225.0731. IR (neat, $\mathbf{c m}^{-1}$ ): 2959, 2924, 2855, 2359, 1766, 1739, 1326, 1204, $1160,1133,1107,984,789$. 
<smiles>COC(=O)C(=O)OC1(C)CCOCC1</smiles><smiles>CC(C(=O)O)C(C)(C)C(=O)O</smiles><smiles>CC1(OC(=O)C(=O)O)CCOCC1</smiles>

Lithium 2-((4-methyltetrahydro-2H-pyran-4-yl)oxy)-2-oxoacetate: A round bottom flask was charged with methyl (4-methyltetrahydro-2H-pyran-4-yl) oxalate (527 mg, $2.61 \mathrm{mmol}, 1.0$ equiv) followed by the addition of THF ( $5 \mathrm{~mL})$. To this solution, $0.5 \mathrm{~N}$ aq. $\mathrm{LiOH}(5.11 \mathrm{~mL}, 2.55 \mathrm{mmol}$, 0.98 equiv) was added dropwise. The mixture was stirred vigorously for $15 \mathrm{~min}$ at room temperature, then concentrated under reduced pressure to give the title compound as a pale yellow solid (502 mg, quantitative yield).

1H NMR: (600 MHz, DMSO-d $\left.{ }_{6}\right) \delta 3.61-3.54(\mathrm{~m}, 4 \mathrm{H}), 2.02-1.96(\mathrm{~m}, 2 \mathrm{H}), 1.65-1.58(\mathrm{~m}, 2 \mathrm{H}), 1.46$ (s, 3H). ${ }^{13}$ C NMR: (151 MHz, DMSO-d 6 ) $\delta 167.1,163.1,76.9,62.3,36.5,24.8$. HRMS (m/z): Calculated for $\mathrm{C}_{8} \mathrm{H}_{11} \mathrm{LiO}_{5}\left([\mathrm{M}-\mathrm{Li}]^{-}\right): 187.0607$, found: 187.0609. IR (neat, cm-1): 2970, 2871, $1652,1228,1138,1101,791$.

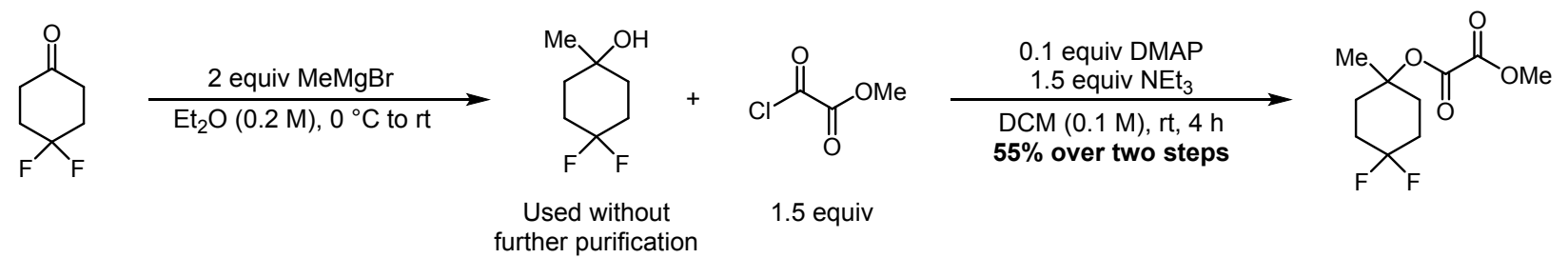

4,4-Difluoro-1-methylcyclohexyl methyl oxalate: A solution of methyl magnesium bromide (2.9 $\mathrm{M}$ in $\mathrm{Et}_{2} \mathrm{O} ; 3.9 \mathrm{~mL}, 11.52 \mathrm{mmol}, 2$ equiv) was added dropwise to 4,4-difluorocyclohexanone (773 $\mathrm{mg}, 5.76 \mathrm{mmol}, 1.0$ equiv) in $\mathrm{Et}_{2} \mathrm{O}(20 \mathrm{~mL})$ at $0{ }^{\circ} \mathrm{C}$. The resulting solution was warmed to room temperature and stirred for 3 hours. Upon completion, the reaction was quenched with a saturated aqueous $\mathrm{NH}_{4} \mathrm{Cl}$ solution $(25 \mathrm{~mL})$ and diluted with $\mathrm{Et}_{2} \mathrm{O}(25 \mathrm{~mL})$. The layers were separated, and the aqueous phase was extracted with $\mathrm{Et}_{2} \mathrm{O}(2 \times 25 \mathrm{~mL})$. The combined $\mathrm{Et}_{2} \mathrm{O}$ phases were washed 
with saturated aqueous $\mathrm{NaHCO}_{3}(25 \mathrm{~mL})$ and $\mathrm{H}_{2} \mathrm{O}(20 \mathrm{~mL})$, dried over $\mathrm{MgSO}_{4}$ and concentrated to yield 4,4-difluoro-1-methylcyclohexan-1-ol which was used in the next step without further purification.

Methyl chlorooxoacetate ( $640 \mu \mathrm{L}, 6.91 \mathrm{mmol}, 1.2$ equiv) was added to a solution of crude 4,4difluoro-1-methylcyclohexan-1-ol, DMAP (71 mg, $0.58 \mathrm{mmol}, 0.1$ equiv) and $\mathrm{NEt}_{3}(970 \mu \mathrm{L}, 6.91$ mmol, 1.2 equiv) in DCM (60 mL) and the resulting solution was stirred at room temperature for 3 hours. Additional methyl chlorooxoacetate $(138 \mu \mathrm{L}, 1.73 \mathrm{mmol}, 0.3$ equiv $)$ and $\mathrm{NEt}_{3}(210 \mu \mathrm{L}$, $1.73 \mathrm{mmol}, 0.3$ equiv) were added, and the reaction mixture was stirred for another hour. Upon completion, the reaction was quenched with a saturated aqueous $\mathrm{NH}_{4} \mathrm{Cl}$ solution $(30 \mathrm{~mL})$. The layers were separated, and the aqueous phase was extracted with DCM $(2 \times 30 \mathrm{~mL})$. The combined organic phases were washed with saturated aqueous $\mathrm{NaHCO}_{3}$ solution $(30 \mathrm{~mL})$ and brine $(30 \mathrm{~mL})$, dried over $\mathrm{MgSO}_{4}$ and concentrated. The crude material was purified by flash column chromatography (Hex $\rightarrow$ 5:1 Hex:EtOAc) to give the title compound as a colorless solid (755 mg, $55 \%$ yield over two steps).

${ }^{1}$ H NMR: $\left(600 \mathrm{MHz}, \mathrm{CDCl}_{3}\right) \delta 3.89(\mathrm{~s}, 3 \mathrm{H}), 2.47-2.41(\mathrm{~m}, 2 \mathrm{H}), 2.02-1.91(\mathrm{~m}, 4 \mathrm{H}), 1.78-1.71(\mathrm{~m}$, 2H), 1.62 (s, 3H). ${ }^{13} \mathrm{C}$ NMR: $\left(151 \mathrm{MHz}, \mathrm{CDCl}_{3}\right) \delta 158.7,156.7,124.2,122.6(\mathrm{~d}, J=4.3 \mathrm{~Hz}), 121.0$, $84.0(\mathrm{~d}, J=1.3 \mathrm{~Hz}), 53.6,32.8$ (d, $J=9.2 \mathrm{~Hz}), 29.7$ (t, $J=24.9 \mathrm{~Hz}), 24.6$ (d, $J=2.0 \mathrm{~Hz})$. Rf: $0.41(4: 1$ Hex:EtOAc). HRMS (m/z): Calculated for $\mathrm{C}_{10} \mathrm{H}_{14} \mathrm{~F}_{2} \mathrm{O}_{4}\left([\mathrm{M}+\mathrm{Na}]^{+}\right)$: 259.0758, found: 259.0761 . IR (neat, $\mathbf{c m}^{-1}$ ): 2945, 1759, 1431, 1323, 1203, 1094, 1029, 887, 792. 
<smiles>COC(=O)C(=O)OC1(C)CCC(F)(F)CC1</smiles>
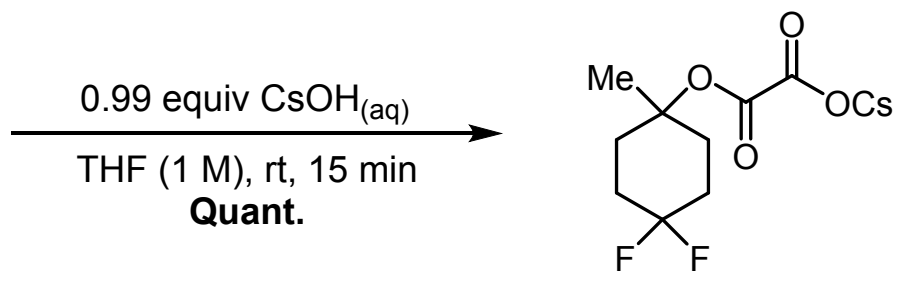

Cesium 2-((4,4-difluoro-1-methylcyclohexyl)oxy)-2-oxoacetate: A round bottom flask was charged with 4,4-difluoro-1-methylcyclohexyl methyl oxalate (735 mg, $3.11 \mathrm{mmol}, 1.0$ equiv) followed by the addition of THF ( $3 \mathrm{~mL})$. To this solution, $1 \mathrm{~N}$ aq. $\mathrm{CsOH}(3.08 \mathrm{~mL}, 3.08 \mathrm{mmol}$, 0.99 equiv) was added dropwise. The mixture was stirred vigorously for $15 \mathrm{~min}$ at room temperature, then concentrated under reduced pressure to give the title compound as a pale yellow solid (1.09 g, quantitative yield).

${ }^{1}$ H NMR: $(600$ MHz, DMSO-d 6 ) $\delta$ 2.26-2.20 (m, 2H), 1.99-1.92 (m, 1H), 1.91-1.84 (m, 3H), 1.601.53 (m, 2H), 1.46 (s, 3H). ${ }^{13}$ C NMR: (151 MHz, DMSO-d 6 ) $\delta 167.4,163.0,125.3,123.7$ (d, $J=2.6 \mathrm{~Hz}), 122.1,77.1,32.5(\mathrm{~d}, J=9.1 \mathrm{~Hz}), 29.2(\mathrm{t}, J=24.2 \mathrm{~Hz}), 24.4(\mathrm{~d}, J=2.1 \mathrm{~Hz}) . \mathbf{H R M S}(\mathbf{m} / \mathbf{z})$ : Calculated for $\mathrm{C}_{9} \mathrm{H}_{11} \mathrm{CsF}_{2} \mathrm{O}_{4}\left([\mathrm{M}-\mathrm{Cs}]^{-}\right): 221.0625$, found: 221.0623. IR (neat, $\left.\mathbf{c m}^{-1}\right):$ 2948, 1717 , $1671,1360,1185,1095,941,779$.

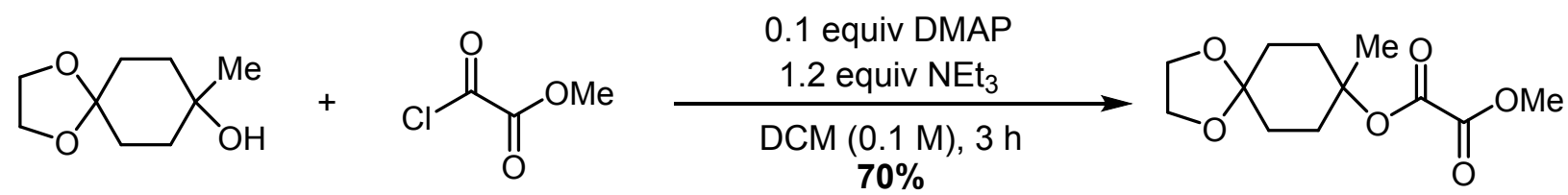

1.2 equiv

Methyl (8-methyl-1,4-dioxaspiro[4.5]decan-8-yl) oxalate: Methyl chlorooxoacetate $(210 \mu \mathrm{L}$, $2.25 \mathrm{mmol}, 1.2$ equiv) was added to a solution of 8-methyl-1,4-dioxaspiro[4.5]decan-8-ol ${ }^{17}$ (323 mg, 1.88 mmol, 1.0 equiv), DMAP (23 mg, 0.19 mmol, 0.1 equiv) and $\mathrm{NEt}_{3}(320 \mu \mathrm{L}, 2.25$ mmol, 1.2 equiv) in DCM (20 mL) and the resulting solution was stirred at room temperature for 3 hours. 
Upon completion, the reaction was quenched with a saturated aqueous $\mathrm{NH}_{4} \mathrm{Cl}$ solution $(20 \mathrm{~mL})$. The layers were separated, and the aqueous phase was extracted with DCM $(2 \times 20 \mathrm{~mL})$. The combined organic phases were washed with saturated aqueous $\mathrm{NaHCO}_{3}$ solution $(20 \mathrm{~mL})$ and brine $(20 \mathrm{~mL})$, dried over $\mathrm{MgSO}_{4}$ and concentrated. The crude material was purified by flash column chromatography (Hex $\rightarrow$ 9:1 Hex:EtOAc) to give the title compound as a pale yellow oil (342 mg, 70\%).

${ }^{1}$ H NMR: $\left(600 \mathrm{MHz}, \mathrm{CDCl}_{3}\right) \delta$ 3.98-3.92 (m, 4H), 2.38-2.33 (m, 2H), 1.83-1.72 (m, 4H), 1.64$1.60(\mathrm{~m}, 2 \mathrm{H}), 1.59(\mathrm{~s}, 3 \mathrm{H}) .{ }^{13} \mathrm{C}$ NMR: $\left(151 \mathrm{MHz}, \mathrm{CDCl}_{3}\right) \delta$ 158.9, 156.9, 108.0, 85.3, 64.5, 64.4, 53.4, 33.9, 30.5, 24.7. Rf: 0.4 (4:1 Hex:EtOAc). HRMS (m/z): Calculated for $\mathrm{C}_{18} \mathrm{H}_{23} \mathrm{~N}$ $\left([\mathrm{M}+\mathrm{Na}]^{+}\right):$281.1001, found: 281.0999. IR (neat, $\left.\mathbf{c m}^{-1}\right):$ 2956, 2885, 2360, 1762, 1739, 1440, $1325,1203,1144,1095,1038,910,790$.

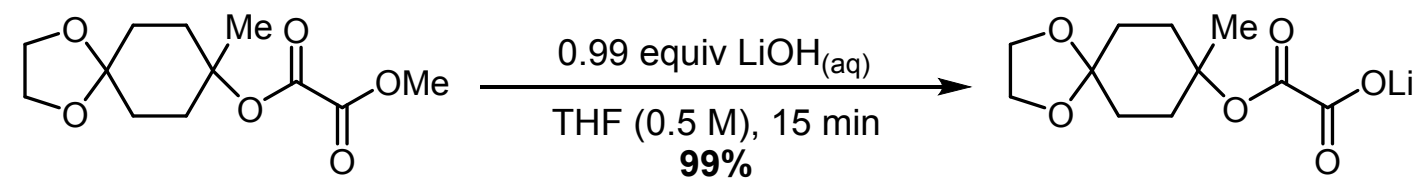

Lithium 2-((8-methyl-1,4-dioxaspiro[4.5]decan-8-yl)oxy)-2-oxoacetate: A round bottom flask was charged with methyl (8-methyl-1,4-dioxaspiro[4.5]decan-8-yl) oxalate (483 mg, $1.87 \mathrm{mmol}$, 1.0 equiv) followed by the addition of THF ( $3 \mathrm{~mL}$ ). To this solution, $0.5 \mathrm{~N}$ aq. $\mathrm{LiOH}(3.7 \mathrm{~mL}, 1.85$ mmol, 0.99 equiv) was added dropwise. The mixture was stirred vigorously for $15 \mathrm{~min}$ at room temperature, then concentrated under reduced pressure to give the title compound as a pale yellow solid (462 mg, quantitative yield).

${ }^{1}$ H NMR: $\left(600 \mathrm{MHz}, \mathrm{DMSO}_{-} \mathrm{d}_{6}\right) \delta 3.86-3.81(\mathrm{~m}, 4 \mathrm{H}), 2.16-2.11(\mathrm{~m}, 2 \mathrm{H}), 1.66(\mathrm{td}, J=12.6,3.7$ $\mathrm{Hz}, 2 \mathrm{H}), 1.55$ (td, $J=13.2,3.6 \mathrm{~Hz}, 2 \mathrm{H}), 1.50-1.45$ (m, 2H), 1.42 (s, 3H). ${ }^{13} \mathbf{C}$ NMR: $(151 \mathrm{MHz}$, DMSO-d $\left._{6}\right) \delta 167.3,163.2,107.5,78.4,63.7,63.6,33.8,30.1,24.8$. HRMS (m/z): Calculated for 
$\mathrm{C}_{11} \mathrm{H}_{15} \mathrm{LiO}_{6}\left([\mathrm{M}-\mathrm{Li}]^{-}\right): 243.0869$, found: 243.0857. IR (neat, $\left.\mathbf{c m}^{-1}\right)$ : 2938, 1707, 1654, 1262 , 1235, 1091, 1038, 908, 802. 


\section{Experimental Procedures for the Photoredox-Catalyzed Minisci Reaction}

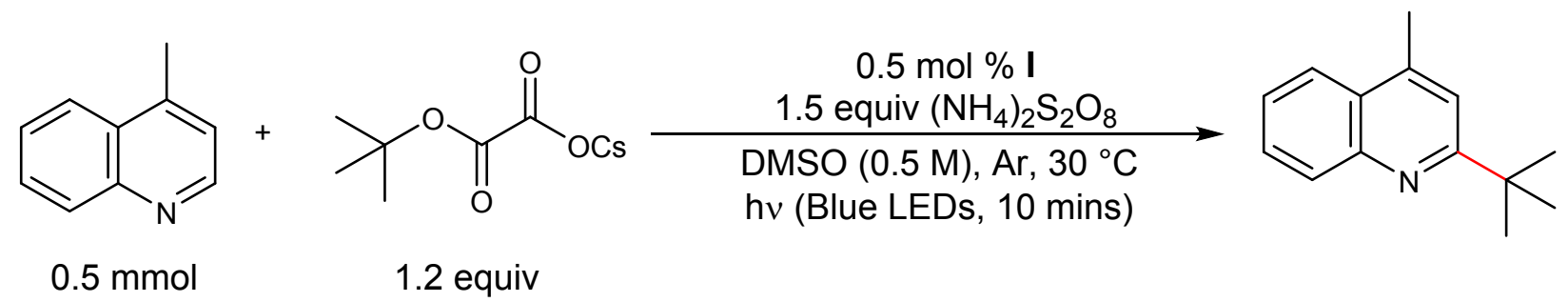

2-(tert-Butyl)-4-methylquinoline (3): Prepared according to GP1 from lepidine (0.5 mmol, 66 $\mu \mathrm{L}, 1$ equiv), cesium 2-(tert-butoxy)-2-oxoacetate ${ }^{2}$ (167 mg, $0.6 \mathrm{mmol}, 1.2$ equiv), $\operatorname{Ir}\left[\mathrm{dF}\left(\mathrm{CF}_{3}\right) \mathrm{ppy}\right]_{2}(\mathrm{dtbbpy}) \mathrm{PF}_{6}(2.8 \mathrm{mg}, 0.0025 \mathrm{mmol}, 0.5 \mathrm{~mol} \%)$ and $\left(\mathrm{NH}_{4}\right)_{2} \mathrm{~S}_{2} \mathrm{O}_{8}(171 \mathrm{mg}, 0.75$ mmol, 1.5 equiv) in $1 \mathrm{~mL}$ of dry DMSO. The reaction was irradiated with two $30 \mathrm{~W}$ Kessil blue LED lamps for $10 \mathrm{~min}$ at $30{ }^{\circ} \mathrm{C}$. Purified by flash column chromatography (Hex $\rightarrow$ 9:1 Hex:EtOAc) to afford the known product 3 as a clear oil in 94\% yield (94 mg).

${ }^{1}$ H NMR: $\left(600 \mathrm{MHz}, \mathrm{CDCl}_{3}\right) \delta 8.06(\mathrm{~d}, J=8.4 \mathrm{~Hz}, 1 \mathrm{H}), 7.95-7.93(\mathrm{~m}, 1 \mathrm{H}), 7.67-7.64(\mathrm{~m}, 1 \mathrm{H})$, 7.50-7.48 (m, 1H), 7.35 (s, 1H), 2.69 (s, $3 \mathrm{H}), 1.46$ (s, 9H). ${ }^{13}$ C NMR: (151 MHz, $\left.\mathrm{CDCl}_{3}\right) \delta$ 169.1, 147.4, 143.7, 130.1, 128.9, 126.7, 125.5, 123.5, 119.0, 38.1, 30.3, $19.1 \mathbf{R}_{\mathbf{f}}: 0.55$ (8:1 Hex:EtOAc) Reference: Chem. Sci. 2017, 8, 3512.<smiles>Cc1ccnc2ccccc12</smiles>

4-Methyl-2-(1-methylcyclohexyl)quinoline (4): Prepared according to GP1 from lepidine (0.5 mmol, $66 \mu \mathrm{L}, 1$ equiv), cesium 2-((1-methylcyclohexyl)oxy)-2-oxoacetate ${ }^{2}(191 \mathrm{mg}, 0.6 \mathrm{mmol}$, 1.2 equiv), $\operatorname{Ir}\left[\mathrm{dF}\left(\mathrm{CF}_{3}\right) \text { ppy }\right]_{2}(\mathrm{dtbbpy}) \mathrm{PF}_{6}(2.8 \mathrm{mg}, 0.0025 \mathrm{mmol}, 0.5 \mathrm{~mol} \%)$ and $\left(\mathrm{NH}_{4}\right)_{2} \mathrm{~S}_{2} \mathrm{O}_{8}(171$ $\mathrm{mg}, 0.75 \mathrm{mmol}, 1.5$ equiv) in $1 \mathrm{~mL}$ of dry DMSO. The reaction was irradiated with two $30 \mathrm{~W}$ 
Kessil blue LED lamps for 10 min at $30^{\circ} \mathrm{C}$. Purified by flash column chromatography (Hex $\rightarrow$ 9:1 Hex:EtOAc) to afford the known product 4 as a clear oil in 95\% yield (114 mg).

${ }^{1}$ H NMR: $\left(600 \mathrm{MHz}, \mathrm{CDCl}_{3}\right) \delta 8.05(\mathrm{~d}, J=8.4 \mathrm{~Hz}, 1 \mathrm{H}), 7.94(\mathrm{dd}, J=8.3,1.0 \mathrm{~Hz}, 1 \mathrm{H})$, 7.66-7.64 (m, 1H), 7.50-7.47 (m, 1H), 7.32 (s, 1H), $2.69(\mathrm{~s}, 3 \mathrm{H}), 2.36-2.34(\mathrm{~m}, 2 \mathrm{H}), 1.65-1.58(\mathrm{~m}, 4 \mathrm{H}), 1.50-$ $1.44(\mathrm{~m}, 4 \mathrm{H}), 1.29$ (s, 3H). ${ }^{13} \mathrm{C}$ NMR: $\left(151 \mathrm{MHz}, \mathrm{CDCl}_{3}\right) \delta$ 168.4, 147.7, 143.6, 130.1, 128.7, 126.6, 125.5, 123.5, 119.5, 41.4, 37.3, 26.5, 23.1, 19.1. $\mathbf{R}_{\mathbf{f}}: 0.55$ (8:1 Hex:EtOAc). Reference: $J$. Am. Chem. Soc. 2017, 139, 2484.<smiles>CC1=C[C+]=CN=C1c1ccccc1</smiles>

$0.5 \mathrm{mmol}$<smiles>CC1(OC(=O)C(=O)O)CCCCC1</smiles>

1.2 equiv

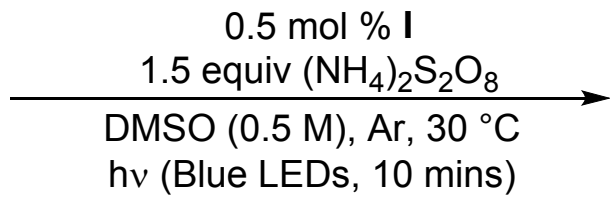

hv (Blue LEDs, 10 mins)<smiles>Cc1cc(C2(C)CCCCC2)nc2ccccc12</smiles>

4-Methyl-2-(1-methylcyclohexyl)quinoline (4): Prepared according to GP1 from lepidine (0.5 mmol, $66 \mu \mathrm{L}, 1$ equiv), lithium 2-((1-methylcyclohexyl)oxy)-2-oxoacetate ${ }^{2}(115 \mathrm{mg}, 0.6 \mathrm{mmol}$, 1.2 equiv), $\operatorname{Ir}\left[\mathrm{dF}\left(\mathrm{CF}_{3}\right) \mathrm{ppy}\right]_{2}(\mathrm{dtbbpy}) \mathrm{PF}_{6}(2.8 \mathrm{mg}, 0.0025 \mathrm{mmol}, 0.5 \mathrm{~mol} \%)$ and $\left(\mathrm{NH}_{4}\right)_{2} \mathrm{~S}_{2} \mathrm{O}_{8}(171$ $\mathrm{mg}, 0.75 \mathrm{mmol}, 1.5$ equiv) in $1 \mathrm{~mL}$ of dry DMSO. The reaction was irradiated with two $30 \mathrm{~W}$ Kessil blue LED lamps for 10 min at $30^{\circ} \mathrm{C}$. Purified by flash column chromatography (Hex $\rightarrow$ 9:1 Hex:EtOAc) to afford the known product 4 as a clear oil in quantitative yield $(120 \mathrm{mg})$. Spectral data matched that previously reported. 


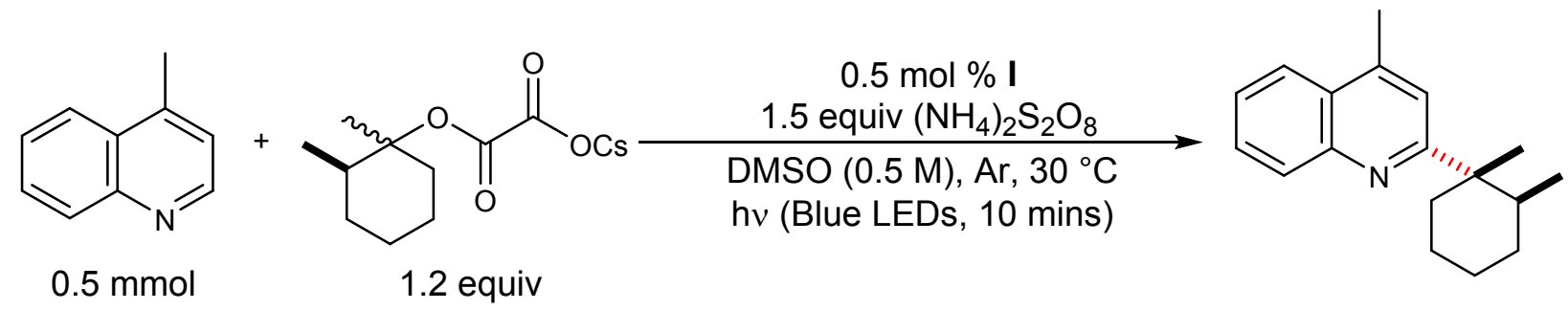

2-((1S, 2S)-1,2-Dimethylcyclohexyl)-4-methylquinoline (5): Prepared according to GP1 from lepidine (0.5 mmol, $66 \mu \mathrm{L}, 1$ equiv), cesium 2-((1,2-dimethylcyclohexyl)oxy)-2-oxoacetate (199

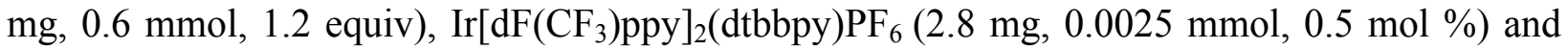
$\left(\mathrm{NH}_{4}\right)_{2} \mathrm{~S}_{2} \mathrm{O}_{8}$ (171 mg, $0.75 \mathrm{mmol}, 1.5$ equiv) in $1 \mathrm{~mL}$ of dry DMSO. The reaction was irradiated with two $30 \mathrm{~W}$ Kessil blue LED lamps for $10 \mathrm{~min}$ at $30{ }^{\circ} \mathrm{C}$. Purified by flash column chromatography (Hex $\rightarrow$ 9:1 Hex:EtOAc) to afford the 5 as a clear oil in 76\% yield with $\mathrm{dr}>20: 1$ (114 mg). The relative configuration of the major diastereomer was assigned by an observed NOESY correlation between the two methyl groups on the cyclohexyl moiety.

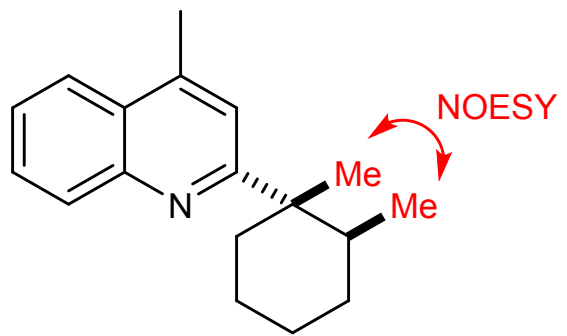

${ }^{1}$ H NMR: $\left(600 \mathrm{MHz}, \mathrm{CDCl}_{3}\right) \delta 8.06(\mathrm{~d}, J=8.4 \mathrm{~Hz}, 1 \mathrm{H}), 7.94(\mathrm{~d}, \mathrm{~J}=8.3 \mathrm{~Hz}, 1 \mathrm{H}), 7.67-7.64(\mathrm{~m}, 1 \mathrm{H})$, 7.50-7.48 (m, 1H), $7.37(\mathrm{~s}, 1 \mathrm{H}), 2.69(\mathrm{~s}, 3 \mathrm{H}), 2.44-2.39(\mathrm{~m}, 1 \mathrm{H}), 1.95$ (dt, J=4.0, $12.9 \mathrm{~Hz}, 1 \mathrm{H})$, 1.81-1.76 (m, 1H), 1.65-1.53 (m, 4H), 1.51-1.36 (m, 2H), $1.42(\mathrm{~s}, 3 \mathrm{H}), 0.63(\mathrm{~d}, J=6.8 \mathrm{~Hz}, 3 \mathrm{H}) .{ }^{13} \mathrm{C}$ NMR: $\left(151 \mathrm{MHz}, \mathrm{CDCl}_{3}\right) \delta 169.1,147.6,143.4,130.2,128.7,126.7,125.4,123.5,119.6,44.8$, 40.2, 38.2, 30.8, 26.4, 22.5, 19.2, 17.2, 16.3. Rf: 0.61 (8:1 Hex:EtOAc). HRMS (m/z): Calculated for $\mathrm{C}_{18} \mathrm{H}_{23} \mathrm{~N}\left([\mathrm{M}+\mathrm{H}]^{+}\right)$: 254.1909, found: 254.1915. IR (neat, $\left.\mathbf{c m}^{-1}\right)$ : 2924, 2854, 2358, 1601, $1557,1507,1445,757$. 
<smiles></smiles>

$0.5 \mathrm{mmol}$<smiles>COC(=O)C(=O)OC1(C)CCCC1</smiles>

1.2 equiv

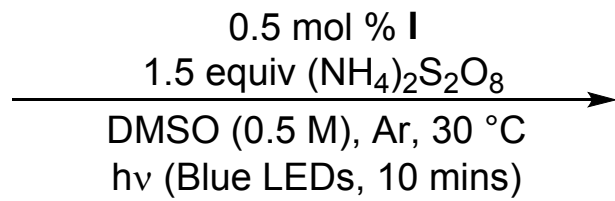

hv (Blue LEDs, 10 mins)

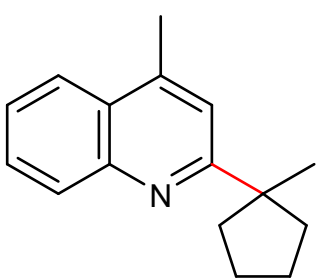

4-Methyl-2-(1-methylcyclopentyl)quinoline (6): Prepared according to GP1 from lepidine $(0.5$ mmol, $66 \mu \mathrm{L}, 1$ equiv), cesium 2-((1-methylcyclopentyl)oxy)-2-oxoacetate ${ }^{2}$ (182 mg, 0.6 mmol, 1.2 equiv $), \operatorname{Ir}\left[\mathrm{dF}\left(\mathrm{CF}_{3}\right) \text { ppy }\right]_{2}(\mathrm{dtbbpy}) \mathrm{PF}_{6}(2.8 \mathrm{mg}, 0.0025 \mathrm{mmol}, 0.5 \mathrm{~mol} \%)$ and $\left(\mathrm{NH}_{4}\right)_{2} \mathrm{~S}_{2} \mathrm{O}_{8}(171$ $\mathrm{mg}, 0.75 \mathrm{mmol}, 1.5$ equiv) in $1 \mathrm{~mL}$ of dry DMSO. The reaction was irradiated with two $30 \mathrm{~W}$ Kessil blue LED lamps for $10 \mathrm{~min}$ at $30{ }^{\circ} \mathrm{C}$. Purified by flash column chromatography (Hex $\rightarrow$ 85:15 Hex:EtOAc) to afford 6 as a clear oil in $85 \%$ yield $(96 \mathrm{mg})$.

${ }^{1}$ H NMR: $\left(600 \mathrm{MHz}, \mathrm{CDCl}_{3}\right) \delta 8.06(\mathrm{~d}, J=8.4 \mathrm{~Hz}, 1 \mathrm{H}), 7.94(\mathrm{dd}, J=8.3,1.1 \mathrm{~Hz}, 1 \mathrm{H})$, 7.66-7.64 (m, 1H), 7.50-7.47 (m, 1H), $7.29(\mathrm{~s}, 1 \mathrm{H}), 2.69(\mathrm{~s}, 3 \mathrm{H}), 2.37-2.31(\mathrm{~m}, 2 \mathrm{H}), 1.86-1.79(\mathrm{~m}, 4 \mathrm{H}), 1.77-$ $1.72(\mathrm{~m}, 2 \mathrm{H}), 1.42$ (s, 3H). ${ }^{13} \mathrm{C}$ NMR: (151 MHz, $\left.\mathrm{CDCl}_{3}\right) \delta 169.1,147.3,143.7,130.0,128.8$, 126.7, 125.5, 123.5, 120.2, 50.2, 39.3, 28.5, 24.7, 19.0. Rf: 0.46 (8:1 Hex:EtOAc) HRMS (m/z): Calculated for $\mathrm{C}_{16} \mathrm{H}_{19} \mathrm{~N}\left([\mathrm{M}+\mathrm{H}]^{+}\right)$: 226.1596, found: 226.1595. IR (neat, $\mathbf{c m}^{-1}$ ): 2954, 2867, 2360, $1603,1558,1507,1446,757$.<smiles>Cc1ccnc2ccccc12</smiles>

$0.5 \mathrm{mmol}$<smiles>O=C(OCl)C(=O)OC12CC3CC(CC(C3)C1)C2</smiles>

1.2 equiv

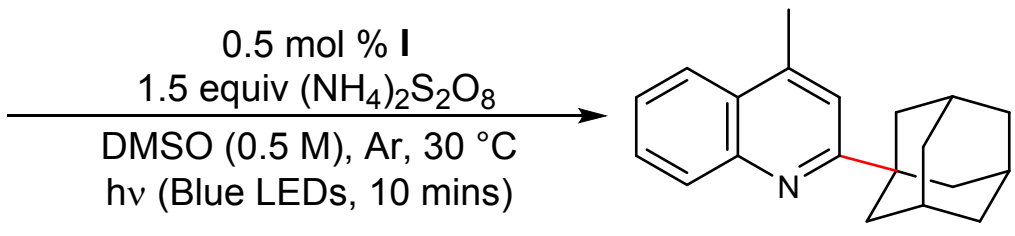

hv (Blue LEDs, 10 mins)

2-((3r, 5r, 7r)-Adamantan-1-yl)-4-methylquinoline (7): Prepared according to GP1 from lepidine $(0.5 \mathrm{mmol}, 66 \mu \mathrm{L}, 1$ equiv), lithium 2-(((3s,5s,7s)-adamantan-1-yl)oxy)-2-oxoacetate (138 mg, $0.6 \mathrm{mmol}, 1.2$ equiv), $\operatorname{Ir}\left[\mathrm{dF}\left(\mathrm{CF}_{3}\right) \mathrm{ppy}\right]_{2}(\mathrm{dtbbpy}) \mathrm{PF}_{6}(2.8 \mathrm{mg}, 0.0025 \mathrm{mmol}, 0.5 \mathrm{~mol} \%)$ 
and $\left(\mathrm{NH}_{4}\right)_{2} \mathrm{~S}_{2} \mathrm{O}_{8}(171 \mathrm{mg}, 0.75 \mathrm{mmol}, 1.5$ equiv) in $1 \mathrm{~mL}$ of dry DMSO. The reaction was irradiated with two $30 \mathrm{~W}$ Kessil blue LED lamps for $10 \mathrm{~min}$ at $30{ }^{\circ} \mathrm{C}$. Purified by flash column chromatography (Hex $\rightarrow$ 9:1 Hex:EtOAc) to afford the known product 7 as a colorless solid in $68 \%$ yield (95 mg).

${ }^{1}$ H NMR: $\left(600 \mathrm{MHz}, \mathrm{CDCl}_{3}\right) \delta 8.06(\mathrm{~d}, J=8.4 \mathrm{~Hz}, 1 \mathrm{H}), 7.94(\mathrm{~d}, J=8.3 \mathrm{~Hz}, 1 \mathrm{H}), 7.66-7.64(\mathrm{~m}, 1 \mathrm{H})$, 7.50-7.47 (m, 1H), $7.32(\mathrm{~s}, 1 \mathrm{H}), 2.17-2.14(\mathrm{~m}, 3 \mathrm{H}), 2.12(\mathrm{~d}, J=2.6 \mathrm{~Hz}, 6 \mathrm{H}), 1.83(\mathrm{t}, J=2.8 \mathrm{~Hz}, 6 \mathrm{H})$. ${ }^{13}$ C NMR: $\left(151 \mathrm{MHz}, \mathrm{CDCl}_{3}\right) \delta 168.8,147.7,143.7,130.1,128.7,126.8,125.5,123.6,118.7$, 41.9, 39.7, 37.0, 29.0, 19.1. Rf: 0.59 (8:1 Hex:EtOAc). Reference: Chem. Sci. 2016, 7, 4754.<smiles>Cc1c[cH+]cnc2ccccc12</smiles>

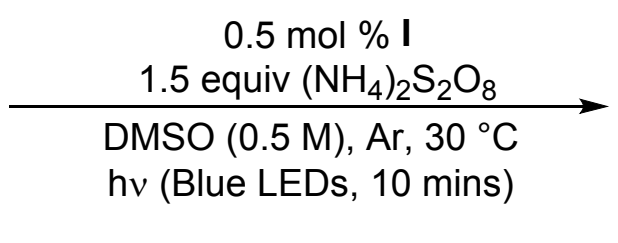

$0.5 \mathrm{mmol}$ 1.2 equiv<smiles>Cc1cc(C2(C)CCN(C(=O)OC(C)(C)C)CC2)nc2ccccc12</smiles>

tert-Butyl 4-methyl-4-(4-methylquinolin-2-yl)piperidine-1-carboxlate (8): Prepared according to GP1 from lepidine ( $0.5 \mathrm{mmol}, 66 \mu \mathrm{L}, 1$ equiv), lithium 2-((1- (tert-butoxycarbonyl)-4methylpiperidin-4-yl)oxy)-2-oxoacetate $\quad\left(\begin{array}{llllll}176 & \mathrm{mg}, & 0.6 & \mathrm{mmol}, & 1.2 & \text { equiv}),\end{array}\right.$ $\operatorname{Ir}\left[\mathrm{dF}\left(\mathrm{CF}_{3}\right) \text { ppy }\right]_{2}(\mathrm{dtbbpy}) \mathrm{PF}_{6}(2.8 \mathrm{mg}, 0.0025 \mathrm{mmol}, 0.5 \mathrm{~mol} \%)$ and $\left(\mathrm{NH}_{4}\right)_{2} \mathrm{~S}_{2} \mathrm{O}_{8}(171 \mathrm{mg}, 0.75$ mmol, 1.5 equiv) in $1 \mathrm{~mL}$ of dry DMSO. The reaction was irradiated with two $30 \mathrm{~W}$ Kessil blue LED lamps for $10 \mathrm{~min}$ at $30{ }^{\circ} \mathrm{C}$. Purified by flash column chromatography (Hex $\rightarrow$ 9:1 Hex:EtOAc) to afford 8 as a clear oil in $70 \%$ yield $(120 \mathrm{mg})$.

'H NMR: (600 MHz, CDCl $) \delta 8.03(\mathrm{~d}, J=8.4 \mathrm{~Hz}, 1 \mathrm{H}), 7.95(\mathrm{~d}, J=8.3 \mathrm{~Hz}, 1 \mathrm{H}), 7.67-7.64(\mathrm{~m}, 1 \mathrm{H})$, 7.52-7.49 (m, 1H), $7.29(\mathrm{~s}, 1 \mathrm{H}), 3.70-3.66(\mathrm{~m}, 2 \mathrm{H}), 3.29-3.25(\mathrm{~m}, 2 \mathrm{H}), 2.69(\mathrm{~s}, 3 \mathrm{H}), 2.47-2.44(\mathrm{~m}$, 2H), 1.75-1.71 (m, 2H), $1.46(\mathrm{~s}, 9 \mathrm{H}), 1.35(\mathrm{~s}, 3 \mathrm{H}) .{ }^{13} \mathrm{C}$ NMR: (151 MHz, $\left.\mathrm{CDCl}_{3}\right) \delta$ 166.1, 155.3, 
147.5, 144.3, 130.1, 129.0, 126.7, 125.9, 123.6, 119.3, 79.2, 39.9, 36.3, 29.2, 28.6, 19.2. Rf: 0.26

(8:1 Hex:EtOAc). HRMS (m/z): Calculated for $\mathrm{C}_{21} \mathrm{H}_{28} \mathrm{~N}_{2} \mathrm{O}_{2}\left([\mathrm{M}+\mathrm{H}]^{+}\right)$: 341.2229, found: 341.2228. IR (neat, $\mathbf{c m}^{-1}$ ): 2970, 2926, 2362, 1668, 1601, 1421, 1248, 1171, 759.<smiles>Cc1ccnc2ccccc12</smiles>

$0.5 \mathrm{mmol}$<smiles>CC1(OC(=O)C(=O)O)CCOCC1</smiles>

1.2 equiv

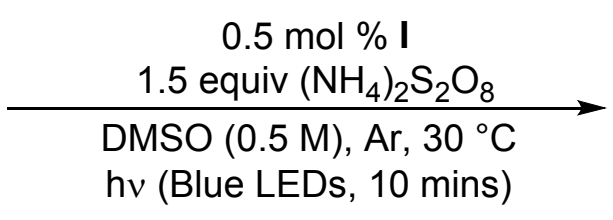

hv (Blue LEDs, 10 mins)<smiles>Cc1cc(C2(C)CCOCC2)nc2ccccc12</smiles>

4-Methyl-2-(4-methyltetrahydro-2H-pyran-4-yl)quinoline (9): Prepared according to GP1 from lepidine (0.5 mmol, $66 \mu \mathrm{L}, 1$ equiv), lithium 2-((4-methyltetrahydro-2H-pyran-4-yl)oxy)-2oxoacetate (116 mg, $0.6 \mathrm{mmol}, 1.2$ equiv), $\operatorname{Ir}\left[\mathrm{dF}\left(\mathrm{CF}_{3}\right) \mathrm{ppy}\right]_{2}(\mathrm{dtbbpy}) \mathrm{PF}_{6}(2.8 \mathrm{mg}, 0.0025 \mathrm{mmol}, 0.5$ $\mathrm{mol} \%)$ and $\left(\mathrm{NH}_{4}\right)_{2} \mathrm{~S}_{2} \mathrm{O}_{8}(171 \mathrm{mg}, 0.75 \mathrm{mmol}, 1.5$ equiv) in $1 \mathrm{~mL}$ of dry DMSO. The reaction was irradiated with two $30 \mathrm{~W}$ Kessil blue LED lamps for $10 \mathrm{~min}$ at $30{ }^{\circ} \mathrm{C}$. Purified by flash column chromatography (Hex $\rightarrow$ 9:1 Hex:EtOAc) to afford 9 as a clear oil in 88\% yield (106 mg).

${ }^{1}$ H NMR: $\left(600 \mathrm{MHz}, \mathrm{CDCl}_{3}\right) \delta 8.04(\mathrm{~d}, J=8.3 \mathrm{~Hz}, 1 \mathrm{H}), 7.96(\mathrm{dd}, J=0.9,8.3 \mathrm{~Hz}, 1 \mathrm{H}), 7.69-7.66$ (m, 1H), 7.53-7.51 (m, 1H), $7.27(\mathrm{~s}, 1 \mathrm{H}), 3.85-3.81(\mathrm{~m}, 2 \mathrm{H}), 3.66-3.62(\mathrm{~m}, 2 \mathrm{H}), 2.70(\mathrm{~s}, 3 \mathrm{H}), 2.50-$ $2.45(\mathrm{~m}, 2 \mathrm{H}), 1.87-1.82(\mathrm{~m}, 2 \mathrm{H}), 1.37(\mathrm{~s}, 3 \mathrm{H}) .{ }^{13} \mathrm{C}$ NMR: $\left(151 \mathrm{MHz}, \mathrm{CDCl}_{3}\right) \delta$ 166.6, 147.6, 144.2, 130.1, 129.0, 126.7, 125.8, 123.6, 119.2, 65.1, 39.2, 37.2, 29.3, 19.2. Rf: 0.25 (8:1 Hex:EtOAc) HRMS (m/z): Calculated for $\mathrm{C}_{16} \mathrm{H}_{19} \mathrm{NO}\left([\mathrm{M}+\mathrm{H}]^{+}\right)$: 242.1545, found: 242.1545. IR (neat, $\mathbf{c m}^{-1}$ ): 2953, 2850, 2359, 1601, 1558, 1506, 1449, 1104, 759. 
<smiles></smiles>

$0.5 \mathrm{mmol}$<smiles>CCOC(=O)C(=O)OC1(C)CCC(F)(F)CC1</smiles>

1.2 equiv

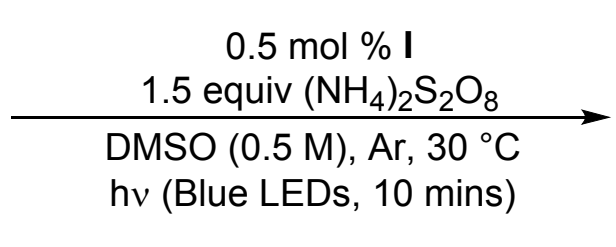

(l)

2-(4,4-Difluoro-1-methylcyclohexyl)-4-methylquinoline (10): Prepared according to GP1 from lepidine (0.5 mmol, $66 \mu \mathrm{L}, 1$ equiv), cesium 2-((4,4-difluoro-1-methylcyclohexyl)oxy)-2oxoacetate (212 mg, $0.6 \mathrm{mmol}, 1.2$ equiv), $\operatorname{Ir}\left[\mathrm{dF}\left(\mathrm{CF}_{3}\right) \text { ppy }\right]_{2}(\mathrm{dtbbpy}) \mathrm{PF}_{6}(2.8 \mathrm{mg}, 0.0025 \mathrm{mmol}, 0.5$ mol \%) and $\left(\mathrm{NH}_{4}\right)_{2} \mathrm{~S}_{2} \mathrm{O}_{8}(171 \mathrm{mg}, 0.75 \mathrm{mmol}, 1.5$ equiv $)$ in $1 \mathrm{~mL}$ of dry DMSO. The reaction was irradiated with two $30 \mathrm{~W}$ Kessil blue LED lamps for $10 \mathrm{~min}$ at $30^{\circ} \mathrm{C}$. Purified by flash column chromatography (Hex $\rightarrow$ 9:1 Hex:EtOAc) to afford $\mathbf{1 0}$ as a clear oil in 78\% yield (108 mg).

1H NMR: $\left(600 \mathrm{MHz}, \mathrm{CDCl}_{3}\right) \delta 8.04(\mathrm{~d}, J=8.4 \mathrm{~Hz}, 1 \mathrm{H}), 7.96(\mathrm{~d}, J=8.3 \mathrm{~Hz}, 1 \mathrm{H}), 7.68(\mathrm{t}, J=7.5 \mathrm{~Hz}$, $1 \mathrm{H}), 7.53(\mathrm{t}, J=7.5 \mathrm{~Hz}, 1 \mathrm{H}), 7.32(\mathrm{~s}, 1 \mathrm{H}), 2.71(\mathrm{~s}, 3 \mathrm{H}), 2.62-2.60(\mathrm{~m}, 1 \mathrm{H}), 2.05-1.96(\mathrm{~m}, 2 \mathrm{H}), 1.95-$ $1.79(\mathrm{~m}, 4 \mathrm{H}), 1.32$ (s, 3H). ${ }^{13} \mathrm{C}$ NMR: $\left(151 \mathrm{MHz}, \mathrm{CDCl}_{3}\right) \delta 165.1,147.6,144.7,130.2,129.1$, 126.7, 126.0, 123.6, 118.8, 40.6, 33.5 (d, $J=9.5 \mathrm{~Hz}), 31.3$ (t, $J=23.9 \mathrm{~Hz}), 30.0,19.2$. Rf: 0.44 (8:1 Hex:EtOAc) HRMS (m/z): Calculated for $\mathrm{C}_{17} \mathrm{H}_{19} \mathrm{~F}_{2} \mathrm{~N}\left([\mathrm{M}+\mathrm{H}]^{+}\right)$: 276.1564, found: 276.1562. IR (neat, $\mathbf{c m}^{-1}$ ): 2959, 2361, 1602, 1559, 1507, 1411, 1369, 1279, 1125, 1081, 1037, 950, 922. 
<smiles>Cc1ccnc2ccccc12</smiles>

$0.5 \mathrm{mmol}$<smiles>COC(=O)C(=O)OC1(C)CCC2(CC1)OCCO2</smiles>

1.2 equiv

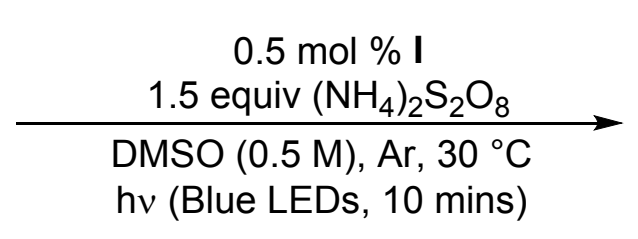

hv (Blue LEDs, 10 mins)

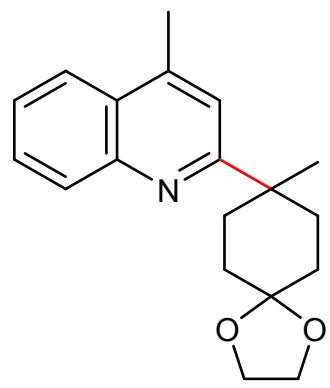

4-Methyl-2-(8-methyl-1,4-dioxaspiro[4.5]decan-8-yl)quinoline (11): Prepared according to

GP1 from lepidine (0.5 mmol, $66 \mu \mathrm{L}, 1$ equiv), lithium 2-((8-methyl-1,4-dioxaspiro[4.5]decan-8yl)oxy)-2-oxoacetate (150 mg, $0.6 \mathrm{mmol}, 1.2$ equiv), $\operatorname{Ir}\left[\mathrm{dF}\left(\mathrm{CF}_{3}\right) \text { ppy }\right]_{2}(\mathrm{dtbbpy}) \mathrm{PF}_{6}(2.8 \mathrm{mg}, 0.0025$ mmol, $0.5 \mathrm{~mol} \%)$ and $\left(\mathrm{NH}_{4}\right)_{2} \mathrm{~S}_{2} \mathrm{O}_{8}(171 \mathrm{mg}, 0.75 \mathrm{mmol}, 1.5$ equiv) in $1 \mathrm{~mL}$ of dry DMSO. The reaction was irradiated with two $30 \mathrm{~W}$ Kessil blue LED lamps for $10 \mathrm{~min}$ at $30{ }^{\circ} \mathrm{C}$. Purified by flash column chromatography (Hex $\rightarrow$ 9:1 Hex:EtOAc) to afford the desired compound as a colorless solid in $82 \%$ yield (122 $\mathrm{mg})$.

${ }^{1}$ H NMR: $\left(600 \mathrm{MHz}, \mathrm{CDCl}_{3}\right) \delta 8.04(\mathrm{~d}, J=8.3 \mathrm{~Hz}, 1 \mathrm{H}), 7.94(\mathrm{~d}, J=8.2 \mathrm{~Hz}, 1 \mathrm{H}), 7.65(\mathrm{t}, J=7.6 \mathrm{~Hz}$, 1H), $7.50(\mathrm{t}, J=7.5 \mathrm{~Hz}, 1 \mathrm{H}), 7.34(\mathrm{~s}, 1 \mathrm{H}), 3.97(\mathrm{t}, J=6.2 \mathrm{~Hz}, 2 \mathrm{H}), 3.90(\mathrm{t}, J=6.2 \mathrm{~Hz}, 2 \mathrm{H}), 2.68(\mathrm{~s}$, 3H), 2.58-2.52 (m, 2H), 1.86-1.79 (m, 2H), 1.75-1.64 (m, 4H), $1.32(\mathrm{~s}, 3 \mathrm{H}) .{ }^{13} \mathrm{C}$ NMR: (151 MHz, $\left.\mathrm{CDCl}_{3}\right) \delta 166.4,147.6,143.9,130.1,128.8,126.6,125.6,109.4,64.3,64.2,40.8,34.6,32.0,29.6$ 19.1. Rf: 0.34 (8:1 Hex:EtOAc) HRMS (m/z): Calculated for $\mathrm{C}_{19} \mathrm{H}_{23} \mathrm{NO}_{2}\left([\mathrm{M}+\mathrm{H}]^{+}\right)$: 298.1807 , found: 298.1818. IR (neat, $\mathbf{c m}^{-1}$ ): 2952, 2359, 1601, 1558, 1506, 1102, 759. 


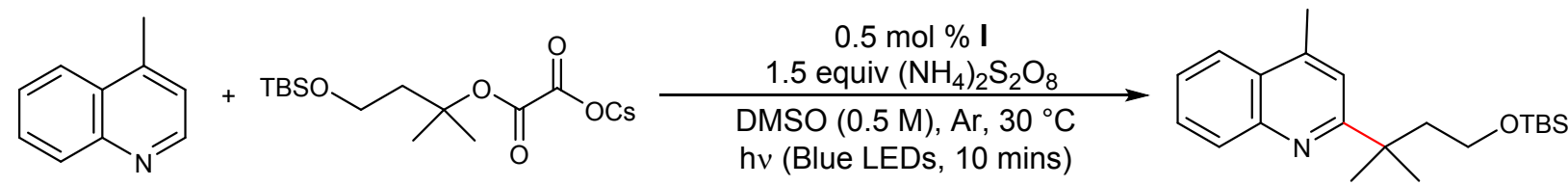

$0.5 \mathrm{mmol}$

1.2 equiv

2-(4-((tert-butyldimethylsilyl)oxy)-2-methylbutan-2-yl)-4-methylquinoline (12): Prepared according to GP1 from lepidine $(0.5 \mathrm{mmol}, 66 \mu \mathrm{L}, 1$ equiv), cesium 2-((4-((tertbutyldimethylsilyl)oxy)-2-methylbutan-2-yl)oxy)-2-oxoacetate ${ }^{2}$ (253 mg, $0.6 \mathrm{mmol}, 1.2$ equiv), $\operatorname{Ir}\left[\mathrm{dF}\left(\mathrm{CF}_{3}\right) \text { ppy }\right]_{2}(\mathrm{dtbbpy}) \mathrm{PF}_{6}(2.8 \mathrm{mg}, 0.0025 \mathrm{mmol}, 0.5 \mathrm{~mol} \%)$ and $\left(\mathrm{NH}_{4}\right)_{2} \mathrm{~S}_{2} \mathrm{O}_{8}(171 \mathrm{mg}, 0.75$ mmol, 1.5 equiv) in $1 \mathrm{~mL}$ of dry DMSO. The reaction was irradiated with two $30 \mathrm{~W}$ Kessil blue LED lamps for $10 \mathrm{~min}$ at $30{ }^{\circ} \mathrm{C}$. Purified by flash column chromatography $(\mathrm{Hex} \rightarrow$ 9:1 Hex:EtOAc) to afford 12 as a clear oil in $81 \%$ yield (139 mg).

${ }^{1}$ H NMR: $\left(600 \mathrm{MHz}, \mathrm{CDCl}_{3}\right) \delta 8.04(\mathrm{dd}, J=8.4 \mathrm{~Hz}, 0.6 \mathrm{~Hz}, 1 \mathrm{H}), 7.94(\mathrm{dd}, J=8.3 \mathrm{~Hz}, 1.0 \mathrm{~Hz}, 1 \mathrm{H})$, 7.67-7.64 (m, 1H), 7.51-7.48 (m, 1H), $7.32(\mathrm{~s}, 1 \mathrm{H}), 3.55-3.53(\mathrm{~m}, 2 \mathrm{H}), 2.68(\mathrm{~d}, J=0.9 \mathrm{~Hz}, 3 \mathrm{H})$, 2.14-2.10 (m, 2H), $1.45(\mathrm{~s}, 6 \mathrm{H}), 0.82(\mathrm{~s}, 9 \mathrm{H}),-0.04(\mathrm{~s}, 6 \mathrm{H}){ }^{13} \mathbf{C}$ NMR: $\left(151 \mathrm{MHz}, \mathrm{CDCl}_{3}\right) \delta 167.6$, $147.4,143.7,130.2,128.8,126.7,125.6,123.5,119.3,60.6,45.8,40.0,28.5,26.1,19.1,18.4$, -5.2. Rf: 0.52 (8:1 Hex:EtOAc) HRMS (m/z): Calculated for $\mathrm{C}_{21} \mathrm{H}_{33} \mathrm{NOSi}\left([\mathrm{M}+\mathrm{H}]^{+}\right): 344.2410$, found: 344.2395. IR (neat, $\mathbf{c m}^{-1}$ ): 2955, 2928, 2856, 2359, 1602, 1558, 1507, 1471, 1254, 1089, 774, 758.

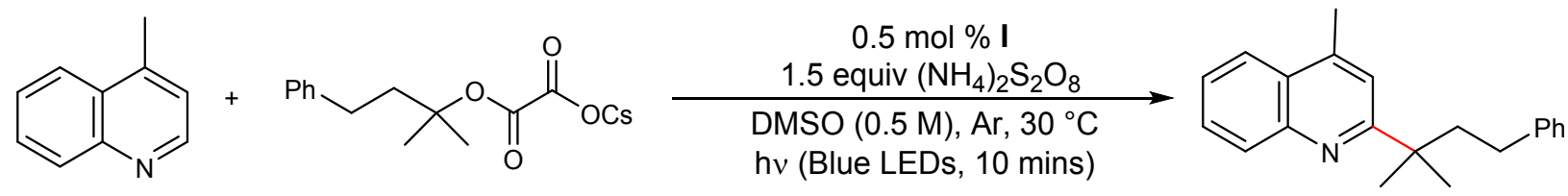

$0.5 \mathrm{mmol} \quad 1.2$ equiv

4-Methyl-2-(2-methyl-4-phenylbutan-2-yl)quinoline (13): Prepared according to GP1 from lepidine (0.5 mmol, $66 \mu \mathrm{L}, 1$ equiv), cesium 2-((2-methyl-4-phenylbutan-2-yl)oxy)-2-oxoacetate ${ }^{2}$ 
(221 mg, $0.6 \mathrm{mmol}, 1.2$ equiv), $\left.\operatorname{Ir}\left[\mathrm{dF}_{(} \mathrm{CF}_{3}\right) \mathrm{ppy}\right]_{2}(\mathrm{dtbbpy}) \mathrm{PF}_{6}(2.8 \mathrm{mg}, 0.0025 \mathrm{mmol}, 0.5 \mathrm{~mol} \%)$ and $\left(\mathrm{NH}_{4}\right)_{2} \mathrm{~S}_{2} \mathrm{O}_{8}(171 \mathrm{mg}, 0.75 \mathrm{mmol}, 1.5$ equiv $)$ in $1 \mathrm{~mL}$ of dry DMSO. The reaction was irradiated with two $30 \mathrm{~W}$ Kessil blue LED lamps for $10 \mathrm{~min}$ at $30{ }^{\circ} \mathrm{C}$. Purified by flash column chromatography (Hex $\rightarrow$ 9:1 Hex:EtOAc) to afford $\mathbf{1 3}$ as a clear oil in 80\% yield (116 mg).

${ }^{1}$ H NMR: $\left(600 \mathrm{MHz}, \mathrm{CDCl}_{3}\right) \delta 8.09(\mathrm{dd}, J=8.4 \mathrm{~Hz}, 0.6 \mathrm{~Hz}, 1 \mathrm{H}), 7.94(\mathrm{dd}, J=8.4 \mathrm{~Hz}, 0.9 \mathrm{~Hz}, 1 \mathrm{H})$, 7.69-7.66 (m, 1H), 7.53-7.50 (m, 1H), 7.35 (s, 1H), 7.25-7.22 (m, 2H), 7.15-7.12 (m, 3H), $2.70(\mathrm{~d}$, $\mathrm{J}=0.8 \mathrm{~Hz}, 3 \mathrm{H}), 2.46-2.43(\mathrm{~m}, 2 \mathrm{H}), 2.16-2.13(\mathrm{~m}, 2 \mathrm{H}), 1.52(\mathrm{~s}, 3 \mathrm{H}) .{ }^{13} \mathrm{C}$ NMR: $\left(151 \mathrm{MHz}, \mathrm{CDCl}_{3}\right)$ $\delta 167.7,147.5,143.8,143.4,130.2,128.8,128.44,128.36,126.7,125.6,125.6,123.6,119.4,45.5$, 41.3, 31.6, 28.1, 19.2. Rf: 0.51 (8:1 Hex:EtOAc) HRMS (m/z): Calculated for $\mathrm{C}_{21} \mathrm{H}_{23} \mathrm{~N}\left([\mathrm{M}+\mathrm{H}]^{+}\right)$: 290.1909, found: 290.1909. IR (neat, $\mathbf{c m}^{-1}$ ): 2960, 2861, 2361, 1601, 1558, 1506, 1452, 758, 698.

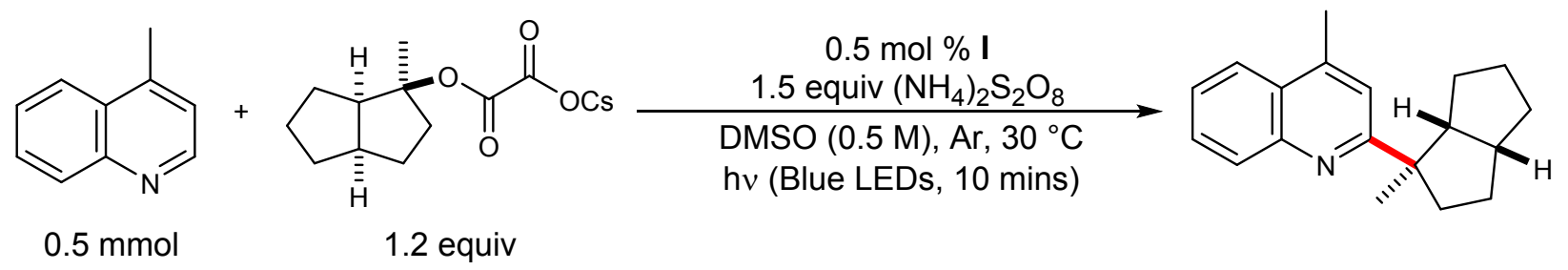

4-Methyl-2-((1R, 3aR, 6aR)-1-methyloctahydropentalen-1-yl)quinoline (14): Prepared according to GP1 from lepidine $(0.5 \mathrm{mmol}, 66 \mu \mathrm{L}, 1$ equiv), cesium 2-(((1S, 3a $R, 6 \mathrm{a} R)-1-$ methyloctahydropentalen-1-yl)oxy)-2-oxoacetate ${ }^{2} \quad(206 \quad \mathrm{mg}, \quad 0.6 \quad \mathrm{mmol}, \quad 1.2$ equiv), $\operatorname{Ir}\left[\mathrm{dF}\left(\mathrm{CF}_{3}\right) \text { ppy }\right]_{2}(\mathrm{dtbbpy}) \mathrm{PF}_{6}(2.8 \mathrm{mg}, 0.0025 \mathrm{mmol}, 0.5 \mathrm{~mol} \%)$ and $\left(\mathrm{NH}_{4}\right)_{2} \mathrm{~S}_{2} \mathrm{O}_{8}(171 \mathrm{mg}, 0.75$ mmol, 1.5 equiv) in $1 \mathrm{~mL}$ of dry DMSO. The reaction was irradiated with two $30 \mathrm{~W}$ Kessil blue LED lamps for $10 \mathrm{~min}$ at $30{ }^{\circ} \mathrm{C}$. Purified by flash column chromatography (Hex $\rightarrow$ 85:15 Hex:EtOAc) to afford $\mathbf{1 4}$ as a clear oil in $47 \%$ yield with $\mathrm{dr}>20: 1(62 \mathrm{mg})$.

${ }^{1}$ H NMR: $\left(600 \mathrm{MHz}, \mathrm{CDCl}_{3}\right) \delta 8.04(\mathrm{dd}, J=8.4 \mathrm{~Hz}, 0.7 \mathrm{~Hz}, 1 \mathrm{H}), 7.93(\mathrm{dd}, J=8.3 \mathrm{~Hz}, 1.0 \mathrm{~Hz}, 1 \mathrm{H})$, 7.66-7.63 (m, 1H), 7.50-7.47 (m, 1H), $7.33(\mathrm{~s}, 1 \mathrm{H}), 3.16(\mathrm{q}, J=8.7 \mathrm{~Hz}, 1 \mathrm{H}), 2.68(\mathrm{~d}, J=0.9 \mathrm{~Hz}, 3 \mathrm{H})$, 
2.48-2.42 (m, 1H), 2.40-2.36 (m, 1H), 1.94-1.89 (m, 1H), 1.84-1.73 (m, 3H), 1.69-1.63 (m, 1H), 1.54-1.44 (m, 2H), 1.35 (s, 3H), 1.30-1.18 (m, 2H). ${ }^{13}$ C NMR: (151 MHz, $\left.\mathrm{CDCl}_{3}\right) \delta$ 169.0, 147.3, $143.5,130.1,128.7,126.7,125.5,123.5,120.1,52.3,52.0,43.0,37.9,35.2,32.0,29.8,28.2,24.7$, 19.1. Rf: 0.58 (8:1 Hex:EtOAc) HRMS (m/z): Calculated for $\mathrm{C}_{19} \mathrm{H}_{23} \mathrm{~N}\left([\mathrm{M}+\mathrm{H}]^{+}\right): 266.1909$, found: 266.1909. IR (neat, $\mathbf{c m}^{-1}$ ): 2943, 2863, 2360, 1604, 1558, 1507, 1447, 757.

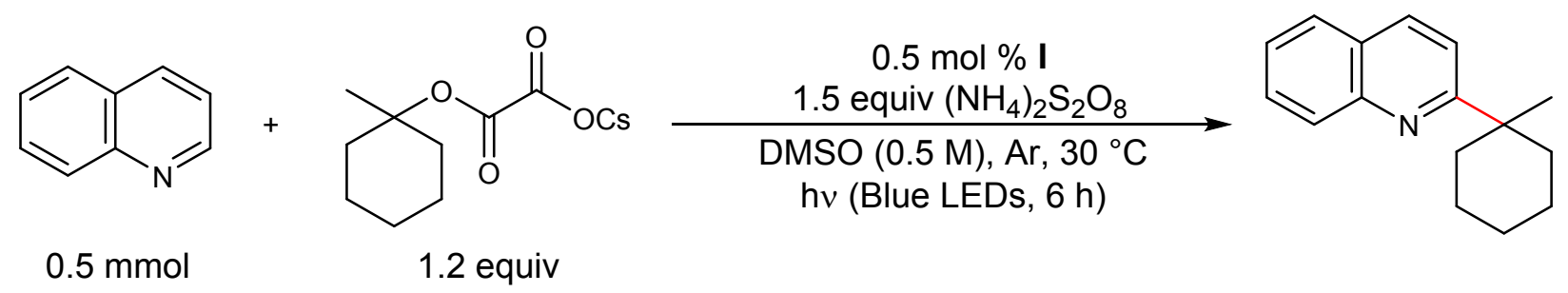

2-(1-methylcyclohexyl)quinoline (20): Prepared according to GP1 from quinoline (59 $\mu \mathrm{L}, 0.5$ mmol, 1 equiv), cesium 2-((1-methylcyclohexyl)oxy)-2-oxoacetate ${ }^{2}(191 \mathrm{mg}, 0.6 \mathrm{mmol}, 1.2$ equiv), $\operatorname{Ir}\left[\mathrm{dF}\left(\mathrm{CF}_{3}\right) \text { ppy }\right]_{2}(\mathrm{dtbbpy}) \mathrm{PF}_{6}(2.8 \mathrm{mg}, 0.0025 \mathrm{mmol}, 0.5 \mathrm{~mol} \%)$ and $\left(\mathrm{NH}_{4}\right)_{2} \mathrm{~S}_{2} \mathrm{O}_{8}(171 \mathrm{mg}$, $0.75 \mathrm{mmol}, 1.5$ equiv) in $1 \mathrm{~mL}$ of dry DMSO. The reaction was irradiated with two $30 \mathrm{~W}$ Kessil blue LED lamps for $6 \mathrm{~h}$ at $30{ }^{\circ} \mathrm{C}$. Purified by flash column chromatography (Hex $\rightarrow$ 9:1 Hex:EtOAc) to afford $\mathbf{2 0}$ as a clear oil in $86 \%$ yield as an inseparable 94:6 mixture of C2:C4 monoalkylated products $(97 \mathrm{mg})$.

1'H NMR: (600 MHz, $\mathrm{CDCl}_{3}$, major C-2 isomer) $\delta$ 8.09-8.04 (m, 2H), $7.77(\mathrm{~d}, J=8.0 \mathrm{~Hz}, 1 \mathrm{H})$, 7.68-7.64 (m, 1H), $7.50(\mathrm{~d}, \mathrm{~J}=8.7 \mathrm{~Hz}, 1 \mathrm{H}), 7.49-7.46(\mathrm{~m}, 1 \mathrm{H}), 2.43-2.32(\mathrm{~m}, 2 \mathrm{H}), 1.70-1.57(\mathrm{~m}$, 4H), 1.52-1.42 (m, 2H), 1.31 (s, 3H). ${ }^{13}$ C NMR: (151 MHz, $\mathrm{CDCl}_{3}$, major C-2 isomer) $\delta 168.7$, 147.9, 135.9, 129.6, 129.0, 127.4, 126.5, 126.3, 125.7, 118.8, 41.6, 37.3, 29.4, 26.5, 23.0. Rf: 0.69 (8:1 Hex:EtOAc). HRMS (m/z): Calculated for $\mathrm{C}_{16} \mathrm{H}_{19} \mathrm{~N}\left([\mathrm{M}+\mathrm{H}]^{+}\right)$: 226.1596, found: 226.1588 . IR (neat, $\mathbf{c m}^{-1}$ ): 3060, 2923, 2359, 1600, 1500, 1445, 827, 754 . 


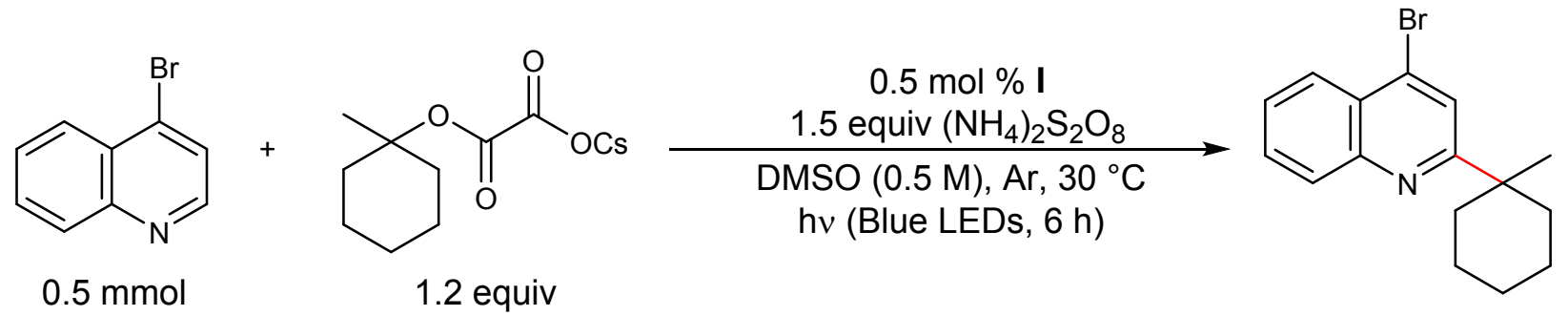

4-Bromo-2-(1-methylcyclohexyl)quinoline (21): Prepared according to GP1 from 4bromoquinoline (104 mg, $0.5 \mathrm{mmol}, 1$ equiv), cesium 2-((1-methylcyclohexyl)oxy)-2-oxoacetate ${ }^{2}$ (191 mg, 0.6 mmol, 1.2 equiv), $\operatorname{Ir}\left[\mathrm{dF}\left(\mathrm{CF}_{3}\right) \text { ppy }\right]_{2}(\mathrm{dtbbpy}) \mathrm{PF}_{6}(2.8 \mathrm{mg}, 0.0025 \mathrm{mmol}, 0.5 \mathrm{~mol} \%)$ and $\left(\mathrm{NH}_{4}\right)_{2} \mathrm{~S}_{2} \mathrm{O}_{8}(171 \mathrm{mg}, 0.75 \mathrm{mmol}, 1.5$ equiv $)$ in $1 \mathrm{~mL}$ of dry DMSO. The reaction was irradiated with two $30 \mathrm{~W}$ Kessil blue LED lamps for $6 \mathrm{~h}$ at $30{ }^{\circ} \mathrm{C}$. Purified by flash column chromatography $($ Hex $\rightarrow$ 9:1 Hex:EtOAc) to afford known 21 as a clear oil in 75\% yield (114 mg).

${ }^{1}$ H NMR: $\left(600 \mathrm{MHz}, \mathrm{CDCl}_{3}\right) \delta 8.13(\mathrm{dd}, J=8.3,0.8 \mathrm{~Hz}, 1 \mathrm{H}), 8.04(\mathrm{~d}, J=8.4 \mathrm{~Hz}, 1 \mathrm{H}), 7.77(\mathrm{~s}, 1 \mathrm{H})$, 7.72-7.69 (m, 1H), 7.58-7.55 (m, 1H), 2.35-2.29 (m, 2H), 1.66-1.60 (m, 4H), 1.49-1.42 (m, 4H), 1.30 (s, 3H). ${ }^{13} \mathrm{C}$ NMR: $\left(151 \mathrm{MHz}, \mathrm{CDCl}_{3}\right) \delta 168.8,148.5,134.1,130.0,127.0,126.5,126.1$, 123.0, 41.7, 37.2, 29.3, 26.4, 23.0. Rf: 0.57 (8:1 Hex:EtOAc). Reference: J. Am. Chem. Soc. 2017, $139,2484$.

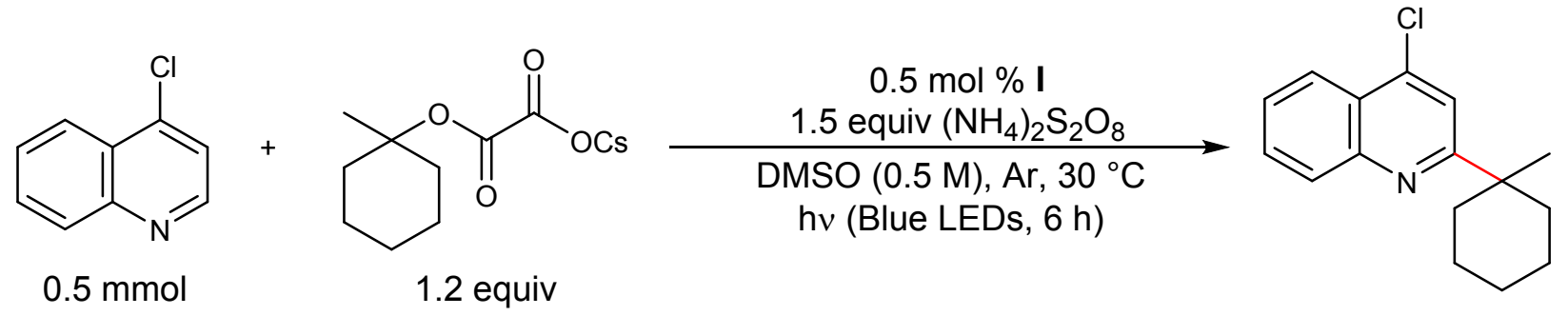
4-Chloro-2-(1-methylcyclohexyl)quinoline (22): Prepared according to GP1 from 4chloroquinoline $\left(66 \mu \mathrm{L}, 0.5 \mathrm{mmol}, 1\right.$ equiv), cesium 2-((1-methylcyclohexyl)oxy)-2-oxoacetate ${ }^{2}$ (191 mg, $0.6 \mathrm{mmol}, 1.2$ equiv), $\operatorname{Ir}\left[\mathrm{dF}\left(\mathrm{CF}_{3}\right) \text { ppy }\right]_{2}(\mathrm{dtbbpy}) \mathrm{PF}_{6}(2.8 \mathrm{mg}, 0.0025 \mathrm{mmol}, 0.5 \mathrm{~mol} \%)$ and $\left(\mathrm{NH}_{4}\right)_{2} \mathrm{~S}_{2} \mathrm{O}_{8}(171 \mathrm{mg}, 0.75 \mathrm{mmol}, 1.5$ equiv $)$ in $1 \mathrm{~mL}$ of dry DMSO. The reaction was irradiated 
with two $30 \mathrm{~W}$ Kessil blue LED lamps for $6 \mathrm{~h}$ at $30^{\circ} \mathrm{C}$. Purified by flash column chromatography $($ Hex $\rightarrow$ 9:1 Hex:EtOAc) to afford known 22 as a clear oil in 84\% yield (109 $\mathrm{mg})$.

${ }^{1}$ H NMR: $\left(600 \mathrm{MHz}, \mathrm{CDCl}_{3}\right) \delta 8.17(\mathrm{dd}, J=8.3,0.8 \mathrm{~Hz}, 1 \mathrm{H}), 8.07(\mathrm{~d}, J=8.4 \mathrm{~Hz}, 1 \mathrm{H}), 7.73-7.70$ (m, 1H), 7.58-7.55 (m, 1H), $7.58(\mathrm{~s}, 1 \mathrm{H}), 2.35-2.30(\mathrm{~m}, 2 \mathrm{H}), 1.66-1.59(\mathrm{~m}, 4 \mathrm{H}), 1.49-1.42(\mathrm{~m}$, 4H), 1.30 (s, 3H). ${ }^{13} \mathrm{C}$ NMR: $\left(151 \mathrm{MHz}, \mathrm{CDCl}_{3}\right) \delta 168.9,148.7,142.5,129.95,129.90,126.7$, 124.7, 123.9, 119.1, 41.8, 37.2, 29.3, 26.4, 23.0. Rf: 0.63 (8:1 Hex:EtOAc). Reference: $J$. Am. Chem. Soc. 2017, 139, 2484.<smiles>[c+]1cc2c(n1)-c1ccccc1C=CC=2</smiles>

$0.5 \mathrm{mmol}$<smiles>CCOC(=O)C(=O)OC1(C)CCCCC1</smiles>

1.2 equiv

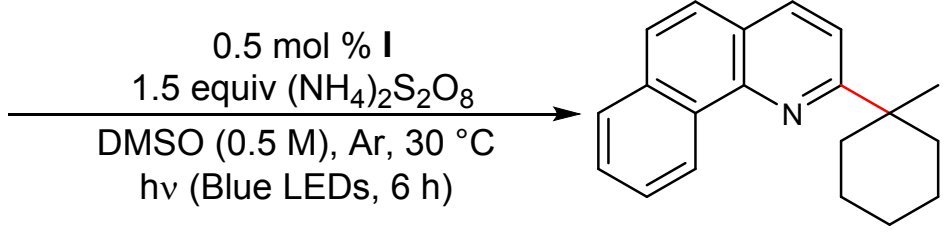

2-(1-Methylcyclohexyl)benzo[ $[\boldsymbol{h}]$ quinoline (23): Prepared according to GP1 from 7,8benzoquinoline ( $90 \mathrm{mg}, 0.5 \mathrm{mmol}, 1$ equiv), cesium 2-((1-methylcyclohexyl)oxy)-2-oxoacetate ${ }^{2}$ (191 mg, $0.6 \mathrm{mmol}, 1.2$ equiv), $\operatorname{Ir}\left[\mathrm{dF}_{(}\left(\mathrm{CF}_{3}\right) \mathrm{ppy}\right]_{2}(\mathrm{dtbbpy}) \mathrm{PF}_{6}(2.8 \mathrm{mg}, 0.0025 \mathrm{mmol}, 0.5 \mathrm{~mol} \%)$ and $\left(\mathrm{NH}_{4}\right)_{2} \mathrm{~S}_{2} \mathrm{O}_{8}(171 \mathrm{mg}, 0.75 \mathrm{mmol}, 1.5$ equiv) in $1 \mathrm{~mL}$ of dry DMSO. The reaction was irradiated with two $30 \mathrm{~W}$ Kessil blue LED lamps for $6 \mathrm{~h}$ at $30^{\circ} \mathrm{C}$. Purified by flash column chromatography $\left(\mathrm{Hex} \rightarrow 9: 1 \mathrm{Hex}:\right.$ EtOAc) to afford $\mathbf{2 3}$ as a clear oil in $46 \%$ yield $(63 \mathrm{mg}){ }^{20}$

${ }^{1}$ H NMR: $\left(600 \mathrm{MHz}, \mathrm{CDCl}_{3}\right) \delta 9.38(\mathrm{~d}, J=8.0 \mathrm{~Hz}, 1 \mathrm{H}), 8.10(\mathrm{~d}, J=8.4 \mathrm{~Hz}, 1 \mathrm{H}), 7.89(\mathrm{~d}, J=7.7 \mathrm{~Hz}$, 1H), $7.76(\mathrm{~d}, J=8.7 \mathrm{~Hz}, 1 \mathrm{H}), 7.73-7.70(\mathrm{~m}, 1 \mathrm{H}), 7.68-7.65(\mathrm{~m}, 2 \mathrm{H}), 7.60(\mathrm{~d}, J=8.4 \mathrm{~Hz}, 1 \mathrm{H}), 2.54-$ $2.47(\mathrm{~m}, 2 \mathrm{H}), 1.72-1.63(\mathrm{~m}, 4 \mathrm{H}), 1.56-1.46(\mathrm{~m}, 4 \mathrm{H}), 1.36(\mathrm{~s}, 3 \mathrm{H}) .{ }^{13} \mathbf{C}$ NMR: $\left(151 \mathrm{MHz}, \mathrm{CDCl}_{3}\right)$ $\delta 167.3,145.4,135.9,133.8,132.1,127.8,126.79,126.75,125.3,124.8,124.0,119.1,41.9,37.6$ 30.1, 26.6, 23.1. Rf: 0.71 (8:1 Hex:EtOAc) HRMS (m/z): Calculated for $\mathrm{C}_{20} \mathrm{H}_{21} \mathrm{~N}\left([\mathrm{M}+\mathrm{H}]^{+}\right)$: 
276.1752, found: 276.1751. IR (neat, $\mathbf{c m}^{-1}$ ): 2952, 2853, 2359, 1595, 1556, 1505, 1446, 1390, $843,756$.<smiles></smiles>

$0.5 \mathrm{mmol}$<smiles>CCOC(=O)C(=O)OC1(C)CCCCC1</smiles>

1.2 equiv

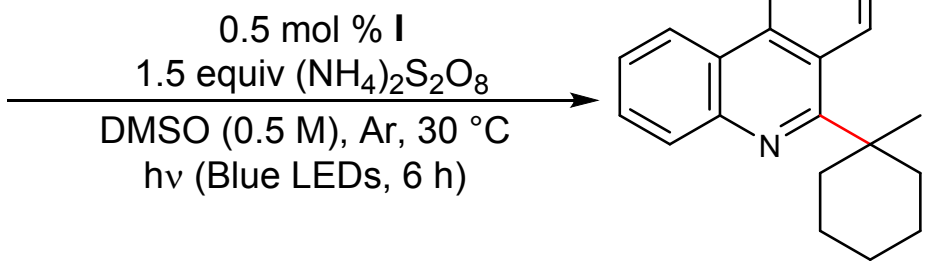

6-(1-Methylcyclohexyl)phenanthridine (24): Prepared according to GP1 from phenanthridine (90 mg, $0.5 \mathrm{mmol}, 1$ equiv), cesium 2-((1-methylcyclohexyl)oxy)-2-oxoacetate ${ }^{2}$ (191 mg, 0.6 mmol, 1.2 equiv), $\operatorname{Ir}\left[\mathrm{dF}\left(\mathrm{CF}_{3}\right) \text { ppy }\right]_{2}(\mathrm{dtbbpy}) \mathrm{PF}_{6}(2.8 \mathrm{mg}, 0.0025 \mathrm{mmol}, 0.5 \mathrm{~mol} \%)$ and $\left(\mathrm{NH}_{4}\right)_{2} \mathrm{~S}_{2} \mathrm{O}_{8}$ (171 mg, $0.75 \mathrm{mmol}, 1.5$ equiv) in $1 \mathrm{~mL}$ of dry DMSO. The reaction was irradiated with two 30 W Kessil blue LED lamps for $6 \mathrm{~h}$ at $30{ }^{\circ} \mathrm{C}$. Purified by flash column chromatography (Hex) to afford known 24 as a clear oil in $33 \%$ yield $(46 \mathrm{mg}){ }^{20}$

1H NMR: $\left(600 \mathrm{MHz}, \mathrm{CDCl}_{3}\right) \delta 8.69(\mathrm{~d}, J=8.2 \mathrm{~Hz}, 1 \mathrm{H}), 8.65(\mathrm{~d}, J=8.5 \mathrm{~Hz}, 1 \mathrm{H}), 8.13(\mathrm{~d}, J=8.0 \mathrm{~Hz})$, 1H), 7.79-7.75 (m, 1H), 7.72-7.68 (m, 1H), 7.63-7.60 (m, 2H), 2.62-2.56 (m, 2H), 1.95-1.89 (m, 2H), $1.70(\mathrm{~s}, 3 \mathrm{H}), 1.67-1.61(\mathrm{~m}, 4 \mathrm{H}), 1.57-1.51(\mathrm{~m}, 1 \mathrm{H}), 1.48-1.42(\mathrm{~m}, 1 \mathrm{H}) .{ }^{13} \mathrm{C}$ NMR: (151 MHz, $\left.\mathrm{CDCl}_{3}\right) \delta 166.3,143.1,134.3,130.4,129.3,128.4,128.2,126.5,125.9,124.6,123.4,123.2,121.7$ 43.7, 39.2, 27.4, 26.7, 23.2. Rf: 0.64 (8:1 Hex:EtOAc). Reference: J. Org. Chem. 2015, 80, 9336. 
<smiles>N#Cc1cccc2cnccc12</smiles>

$0.5 \mathrm{mmol}$<smiles>COC(=O)C(=O)OC1(C)CCCCC1</smiles>

1.2 equiv

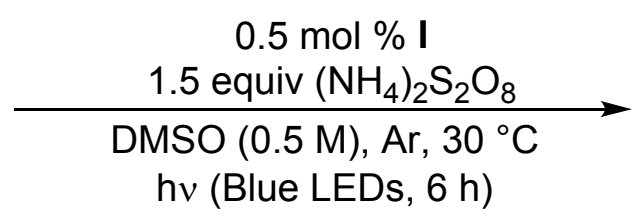

hv (Blue LEDs, $6 \mathrm{~h}$ )

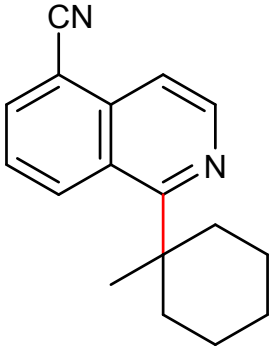

1-(1-Methylcyclohexyl)isoquinoline-5-carbonitrile (25): Prepared according to GP1 from 5cyanoisoquinoline (77 mg, $0.5 \mathrm{mmol}, 1$ equiv), cesium 2-((1-methylcyclohexyl)oxy)-2oxoacetate $^{2}$ (191 mg, $0.6 \mathrm{mmol}, 1.2$ equiv), $\operatorname{Ir}\left[\mathrm{dF}\left(\mathrm{CF}_{3}\right) \text { ppy }\right]_{2}(\mathrm{dtbbpy}) \mathrm{PF}_{6}(2.8 \mathrm{mg}, 0.0025 \mathrm{mmol}$, $0.5 \mathrm{~mol} \%)$ and $\left(\mathrm{NH}_{4}\right)_{2} \mathrm{~S}_{2} \mathrm{O}_{8}(171 \mathrm{mg}, 0.75 \mathrm{mmol}, 1.5$ equiv) in $1 \mathrm{~mL}$ of dry DMSO. The reaction was irradiated with two $30 \mathrm{~W}$ Kessil blue LED lamps for $6 \mathrm{~h}$ at $30{ }^{\circ} \mathrm{C}$. Purified by flash column chromatography (Hex $\rightarrow$ 9:1 Hex:EtOAc) to afford 25 as a clear oil in 26\% yield (32 mg). ${ }^{20}$

${ }^{1}$ H NMR: $\left(600 \mathrm{MHz}, \mathrm{CDCl}_{3}\right) \delta 8.84(\mathrm{~d}, J=8.8 \mathrm{~Hz}, 1 \mathrm{H}), 8.66(\mathrm{~d}, J=4.9 \mathrm{~Hz}, 1 \mathrm{H}), 8.04(\mathrm{~d}, J=6.8 \mathrm{~Hz})$, $7.91(\mathrm{~d}, J=5.0 \mathrm{~Hz}, 1 \mathrm{H}), 7.58(\mathrm{t}, J=7.8 \mathrm{~Hz}, 1 \mathrm{H}), 2.43-2.36(\mathrm{~m}, 2 \mathrm{H}), 1.93-1.87(\mathrm{~m}, 2 \mathrm{H}), 1.67-1.61$ (m, 2H), $1.64(\mathrm{~s}, 3 \mathrm{H}), 1.59-1.51(\mathrm{~m}, 3 \mathrm{H}), 1.46-1.38(\mathrm{~m}, 1 \mathrm{H}) .{ }^{13} \mathbf{C}$ NMR: $\left(151 \mathrm{MHz}, \mathrm{CDCl}_{3}\right) \delta$ $168.5,143.2,137.2,135.3,132.3,126.0,124.8,117.3,116.4,111.1,43.7,39.2,27.3,26.5,23.0$. Rf: 0.30 (8:1 Hex:EtOAc) HRMS (m/z): Calculated for $\mathrm{C}_{17} \mathrm{H}_{18} \mathrm{~N}_{2}\left([\mathrm{M}+\mathrm{H}]^{+}\right)$: 251.1548 , found: 251.1549. IR (neat, $\mathbf{c m}^{-1}$ ): 2942, 2853, 2362, 2225, 1558, 1456, 820, 765.<smiles>c1ccncc1</smiles>

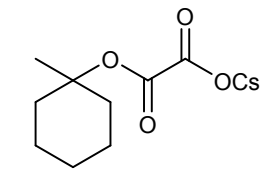

1.2 equiv

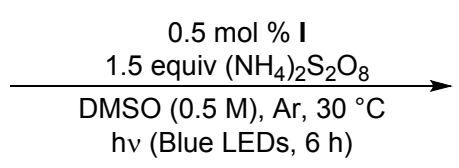

$0.5 \mathrm{mmol}$

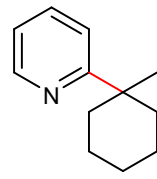

26<smiles>CC1(c2ccncc2)CCCCC1</smiles><smiles>CC1(c2ccnc(C3(C)CCCCC3)c2)CCCCC1</smiles>

Reaction of pyridine to form a mixture of 2-(1-methylcyclohexyl)pyridine (26), 4-(1methylcyclohexyl)pyridine, and 2,4-di-(1-methylcyclohexyl)pyridine: Prepared according to GP1 from pyridine (40 $\mu \mathrm{L}, 0.5 \mathrm{mmol}, 1$ equiv), cesium 2-((1-methylcyclohexyl)oxy)-2- 
oxoacetate $^{2}\left(191 \mathrm{mg}, 0.6 \mathrm{mmol}, 1.2\right.$ equiv), $\operatorname{Ir}\left[\mathrm{dF}\left(\mathrm{CF}_{3}\right) \mathrm{ppy}\right]_{2}(\mathrm{dtbbpy}) \mathrm{PF}_{6}(2.8 \mathrm{mg}, 0.0025 \mathrm{mmol}$, $0.5 \mathrm{~mol} \%)$ and $\left(\mathrm{NH}_{4}\right)_{2} \mathrm{~S}_{2} \mathrm{O}_{8}(171 \mathrm{mg}, 0.75 \mathrm{mmol}, 1.5$ equiv) in $1 \mathrm{~mL}$ of dry DMSO. The reaction was irradiated with two $30 \mathrm{~W}$ Kessil blue LED lamps for $6 \mathrm{~h}$ at $30^{\circ} \mathrm{C}$. The three major products were separated by flash column chromatography (Hex $\rightarrow$ 9:1 Hex:EtOAc) to afford:

2-(1-methylcyclohexyl)pyridine (26) as a clear oil in 31\% yield (27 mg):

${ }^{1}$ H NMR: $\left(600 \mathrm{MHz}, \mathrm{CDCl}_{3}\right) \delta 8.59(\mathrm{~d}, J=3.9 \mathrm{~Hz}, 1 \mathrm{H}), 7.62(\mathrm{dt}, J=7.7,1.8 \mathrm{~Hz}, 1 \mathrm{H}), 7.33(\mathrm{~d}, J=8.1$ $\mathrm{Hz}, 1 \mathrm{H}), 7.10-7.06(\mathrm{~m}, 1 \mathrm{H}), 2.22-2.14(\mathrm{~m}, 2 \mathrm{H}), 1.61-1.53(\mathrm{~m}, 4 \mathrm{H}), 1.46-1.34(\mathrm{~m}, 4 \mathrm{H}), 1.22(\mathrm{~s}$ 3H). ${ }^{13}$ C NMR: $\left(151 \mathrm{MHz}, \mathrm{CDCl}_{3}\right) \delta 168.6,148.9,136.4,120.6,120.3,40.9,37.3,29.2,26.4$, 22.9. Rf: 0.40 (8:1 Hex:EtOAc). HRMS (m/z): Calculated for $\mathrm{C}_{12} \mathrm{H}_{17} \mathrm{~N}\left([\mathrm{M}+\mathrm{H}]^{+}\right)$: 176.1439, found: 176.1438. IR (neat, $\mathbf{c m}^{-1}$ ): 2926, 2856, 2360, 1588, 1470, 1429, 787, 746.

4-(1-methylcyclohexyl)pyridine as a clear oil in 30\% yield $(26 \mathrm{mg})$ :

${ }^{1}$ H NMR: $\left(600 \mathrm{MHz}, \mathrm{CDCl}_{3}\right) \delta 8.52-8.50(\mathrm{~m}, 2 \mathrm{H}), 7.28-7.25(\mathrm{~m}, 2 \mathrm{H}), 2.01-1.94(\mathrm{~m}, 2 \mathrm{H}), 1.59-$ $1.52(\mathrm{~m}, 4 \mathrm{H}), 1.46-1.33(\mathrm{~m}, 4 \mathrm{H}), 1.16(\mathrm{~s}, 3 \mathrm{H}) .{ }^{13} \mathrm{C}$ NMR: $\left(151 \mathrm{MHz}, \mathrm{CDCl}_{3}\right) \delta$ 159.3, 149.8, 121.6, 38.2, 37.3, 30.1, 26.2, 22.6. Rf: 0.07 (8:1 Hex:EtOAc). HRMS (m/z): Calculated for $\mathrm{C}_{12} \mathrm{H}_{17} \mathrm{~N}$ $\left([\mathrm{M}+\mathrm{H}]^{+}\right):$176.1439, found: 176.1434. IR (neat, $\left.\mathbf{c m}^{-1}\right):$ 2929, 2858, 2358, 1595, 1492, 1409, 819.

2,4-di-(1-methylcyclohexyl)pyridine as a clear oil in 11\% yield from pyridine (16 mg).

${ }^{1}$ H NMR: $\left(600 \mathrm{MHz}, \mathrm{CDCl}_{3}\right) \delta 8.50(\mathrm{~d}, \mathrm{~J}=5.3 \mathrm{~Hz}, 1 \mathrm{H}), 7.31(\mathrm{~s}, 1 \mathrm{H}), 7.07(\mathrm{dd}, \mathrm{J}=5.3,1.5 \mathrm{~Hz}, 1 \mathrm{H})$, 2.20-2.14 (m, 2H), 2.00-1.94 (m, 2H), 1.60-1.53 (m, 8H), 1.46-1.35 (m, 8H), $1.22(\mathrm{~s}, 3 \mathrm{H}), 1.16(\mathrm{~s}$ 3H). ${ }^{13}$ C NMR: $\left(151 \mathrm{MHz}, \mathrm{CDCl}_{3}\right) \delta 168.3,159.3,148.7,118.4,117.8,40.9,38.3,37.5,37.4$, 30.1, 29.3, 26.5, 26.3, 22.9, 22.6. Rf: 0.40 (8:1 Hex:EtOAc). HRMS (m/z): Calculated for $\mathrm{C}_{19} \mathrm{H}_{29} \mathrm{~N}\left([\mathrm{M}+\mathrm{H}]^{+}\right): 272.2378$, found: 272.2379. IR (neat, $\left.\mathbf{c m}^{-1}\right): 2925,2856,2359,1595,1549$, $1447,835$. 


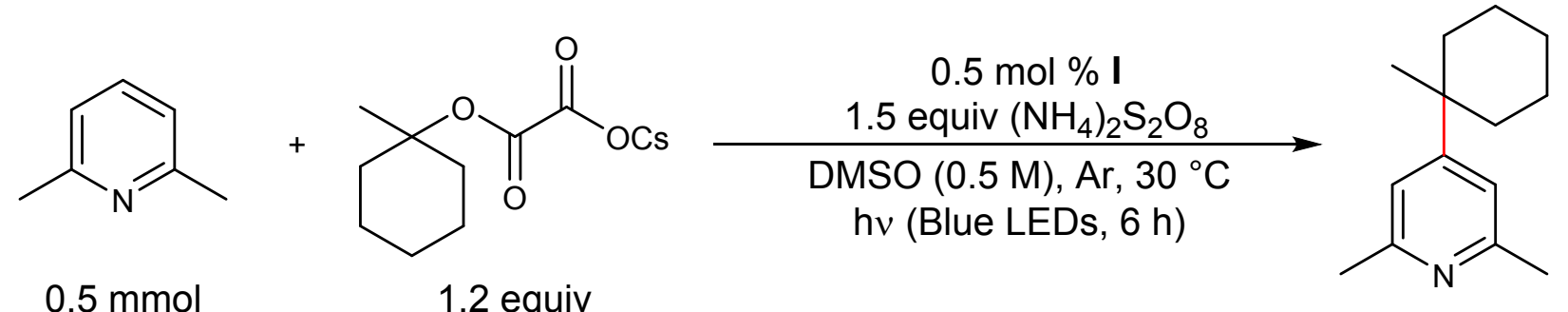

2,6-Dimethyl-4-(1-methylcyclohexyl)pyridine (27): Prepared according to GP1 from 2,6lutidine (58 $\mu \mathrm{L}, 0.5 \mathrm{mmol}, 1$ equiv), cesium 2-((1-methylcyclohexyl)oxy)-2-oxoacetate ${ }^{2}$ (191 mg, $0.6 \mathrm{mmol}, 1.2$ equiv), $\operatorname{Ir}\left[\mathrm{dF}\left(\mathrm{CF}_{3}\right) \text { ppy }\right]_{2}(\mathrm{dtbbpy}) \mathrm{PF}_{6}(2.8 \mathrm{mg}, 0.0025 \mathrm{mmol}, 0.5 \mathrm{~mol} \%)$ and $\left(\mathrm{NH}_{4}\right)_{2} \mathrm{~S}_{2} \mathrm{O}_{8}$ (171 mg, $0.75 \mathrm{mmol}, 1.5$ equiv) in $1 \mathrm{~mL}$ of dry DMSO. The reaction was irradiated with two $30 \mathrm{~W}$ Kessil blue LED lamps for $6 \mathrm{~h}$ at $30^{\circ} \mathrm{C}$. Purified by flash column chromatography $(\mathrm{Hex} \rightarrow$ 9:1 Hex:EtOAc) to afford known 27 as a clear oil in 58\% yield (59 mg).

1H NMR: $\left(600 \mathrm{MHz}, \mathrm{CDCl}_{3}\right) \delta 6.92(\mathrm{~s}, 2 \mathrm{H}), 2.50(\mathrm{~s}, 6 \mathrm{H}), 2.00-1.90(\mathrm{~m}, 2 \mathrm{H}), 1.60-1.49(\mathrm{~m}, 4 \mathrm{H})$, 1.45-1.33 (m, 4H), 1.13 (s, 3H). ${ }^{13}$ C NMR: (151 MHz, $\left.\mathrm{CDCl}_{3}\right) \delta$ 159.8, 157.6, 118.0, 38.0, 37.4, 30.1, 26.3, 24.8, 22.7. Rf: 0.13 (8:1 Hex:EtOAc). Reference: J. Am. Chem. Soc. 2016, 138, 8718.

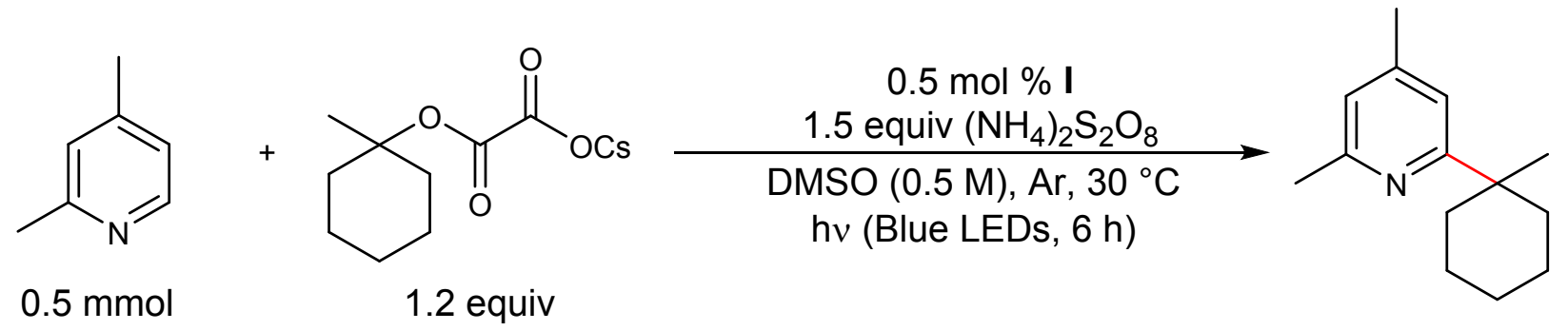

2,4-Dimethyl-6-(1-methylcyclohexyl)pyridine (28): Prepared according to GP1 from 2,4lutidine (58 $\mu \mathrm{L}, 0.5 \mathrm{mmol}, 1$ equiv), cesium 2-((1-methylcyclohexyl)oxy)-2-oxoacetate ${ }^{2}$ (191 mg, $0.6 \mathrm{mmol}, 1.2$ equiv), $\operatorname{Ir}\left[\mathrm{dF}\left(\mathrm{CF}_{3}\right) \mathrm{ppy}\right]_{2}(\mathrm{dtbbpy}) \mathrm{PF}_{6}(2.8 \mathrm{mg}, 0.0025 \mathrm{mmol}, 0.5 \mathrm{~mol} \%)$ and $\left(\mathrm{NH}_{4}\right)_{2} \mathrm{~S}_{2} \mathrm{O}_{8}(171 \mathrm{mg}, 0.75 \mathrm{mmol}, 1.5$ equiv) in $1 \mathrm{~mL}$ of dry DMSO. The reaction was irradiated 
with two $30 \mathrm{~W}$ Kessil blue LED lamps for $6 \mathrm{~h}$ at $30^{\circ} \mathrm{C}$. Purified by flash column chromatography $(\mathrm{Hex} \rightarrow$ 9:1 Hex:EtOAc) to afford 28 as a clear oil in $44 \%$ yield $(45 \mathrm{mg})$.

${ }^{1}$ H NMR: $\left(600 \mathrm{MHz}, \mathrm{CDCl}_{3}\right) \delta 6.91(\mathrm{~s}, 1 \mathrm{H}), 6.75(\mathrm{~s}, 1 \mathrm{H}), 2.48(\mathrm{~s}, 3 \mathrm{H}), 2.28(\mathrm{~s}, 3 \mathrm{H}), 2.17-2.10(\mathrm{~m}$, 2H), 1.58-1.50 (m, 4H), 1.48-1.36 (m, 4H), $1.20(\mathrm{~s}, 3 \mathrm{H}) .{ }^{13} \mathbf{C}$ NMR: $\left(151 \mathrm{MHz}, \mathrm{CDCl}_{3}\right) \delta$ 168.0, 157.1, 146.8, 120.9, 117.8, 40.5, 37.3, 29.0, 26.5, 24.8, 23.0, 21.3. Rf: 0.65 (8:1 Hex:EtOAc) HRMS (m/z): Calculated for $\mathrm{C}_{14} \mathrm{H}_{21} \mathrm{~N}\left([\mathrm{M}+\mathrm{H}]^{+}\right)$: 204.1752, found: 204.1752. IR (neat, $\left.\mathbf{c m}^{-1}\right)$ : 2923, 2854, 2362, 1604, 1569, 1446, 843.
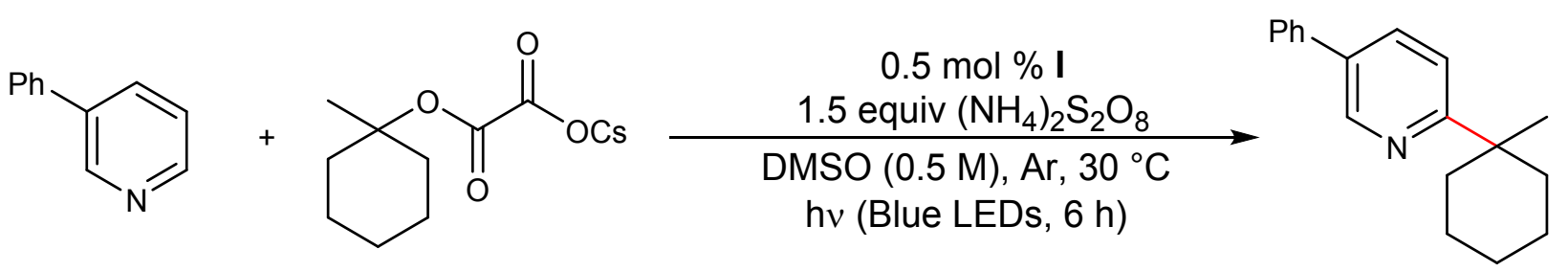

$0.5 \mathrm{mmol} \quad 1.2$ equiv

2-(1-Methylcyclohexyl)-5-phenylpyridine (29): Prepared according to GP1 from 3phenylpyridine ( $71 \mu \mathrm{L}, 0.5 \mathrm{mmol}, 1$ equiv), cesium 2-((1-methylcyclohexyl)oxy)-2-oxoacetate ${ }^{2}$ (191 mg, $0.6 \mathrm{mmol}, 1.2$ equiv), $\operatorname{Ir}\left[\mathrm{dF}_{(}\left(\mathrm{CF}_{3}\right) \mathrm{ppy}\right]_{2}(\mathrm{dtbbpy}) \mathrm{PF}_{6}(2.8 \mathrm{mg}, 0.0025 \mathrm{mmol}, 0.5 \mathrm{~mol} \%)$ and $\left(\mathrm{NH}_{4}\right)_{2} \mathrm{~S}_{2} \mathrm{O}_{8}(171 \mathrm{mg}, 0.75 \mathrm{mmol}, 1.5$ equiv) in $1 \mathrm{~mL}$ of dry DMSO. The reaction was irradiated with two $30 \mathrm{~W}$ Kessil blue LED lamps for $6 \mathrm{~h}$ at $30^{\circ} \mathrm{C}$. Purified by flash column chromatography $\left(\mathrm{Hex} \rightarrow\right.$ 9:1 Hex:EtOAc) to afford $\mathbf{2 9}$ as a clear oil in $82 \%$ yield $(103 \mathrm{mg}) .{ }^{20}$

${ }^{1} \mathbf{H}$ NMR: $\left(600 \mathrm{MHz}, \mathrm{CDCl}_{3}\right) \delta 8.83(\mathrm{~d}, J=2.4 \mathrm{~Hz}, 1 \mathrm{H}), 7.82(\mathrm{dd}, J=8.3 \mathrm{~Hz}, 1 \mathrm{H}), 7.60-7.57(\mathrm{~m}$, 2H), 7.48-7.45 (m, 2H), 7.41-7.36 (m, 2H), 2.27-2.19 (m, 2H), 1.65-1.56 (m, 4H), 1.51-1.40 (m, 4H), 1.27 (s, 3H). ${ }^{13} \mathrm{C}$ NMR: (151 MHz, $\left.\mathrm{CDCl}_{3}\right) \delta$ 167.6, 147.3, 138.2, 134.7, 133.4, 129.1, 127.8, 127.1, 120.1, 40.8, 37.3, 29.3, 26.5, 22.9. Rf: 0.52 (8:1 Hex:EtOAc) HRMS (m/z): Calculated for $\mathrm{C}_{18} \mathrm{H}_{21} \mathrm{~N}\left([\mathrm{M}+\mathrm{H}]^{+}\right): 252.1752$, found: 252.1753. IR (neat, $\left.\mathbf{c m}^{-1}\right):$ 2942, 2855, 2359, 1595, 1473, $1447,752,696$. 


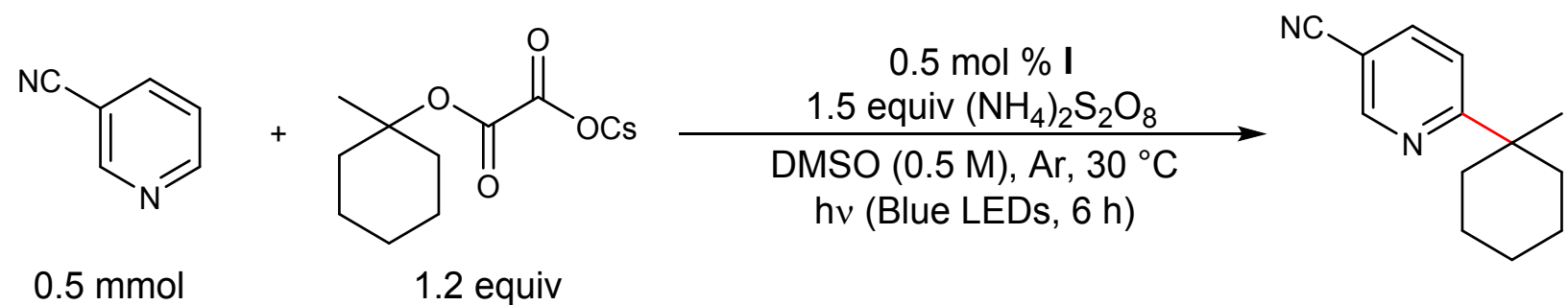

6-(1-Methylcyclohexyl)nicotinonitrile (30): Prepared according to GP1 from 3-cyanopyridine (52 mg, $0.5 \mathrm{mmol}, 1$ equiv), cesium 2-((1-methylcyclohexyl)oxy)-2-oxoacetate ${ }^{2}$ (191 mg, 0.6 mmol, 1.2 equiv), $\operatorname{Ir}\left[\mathrm{dF}\left(\mathrm{CF}_{3}\right) \mathrm{ppy}\right]_{2}(\mathrm{dtbbpy}) \mathrm{PF}_{6}(2.8 \mathrm{mg}, 0.0025 \mathrm{mmol}, 0.5 \mathrm{~mol} \%)$ and $\left(\mathrm{NH}_{4}\right)_{2} \mathrm{~S}_{2} \mathrm{O}_{8}$ (171 mg, $0.75 \mathrm{mmol}, 1.5$ equiv) in $1 \mathrm{~mL}$ of dry DMSO. The reaction was irradiated with two 30 W Kessil blue LED lamps for $6 \mathrm{~h}$ at $30^{\circ} \mathrm{C}$. Purified by flash column chromatography (Hex $\rightarrow$ 9:1 Hex:EtOAc) to afford $\mathbf{3 0}$ as a clear oil in $68 \%$ yield $(68 \mathrm{mg}) .{ }^{20}$

${ }^{1}$ H NMR: $\left(600 \mathrm{MHz}, \mathrm{CDCl}_{3}\right) \delta 8.84(\mathrm{~d}, J=1.6 \mathrm{~Hz}, 1 \mathrm{H}), 7.88(\mathrm{dd}, J=8.4,2.2 \mathrm{~Hz}, 1 \mathrm{H}), 7.45(\mathrm{~d}$, $J=8.4 \mathrm{~Hz}, 1 \mathrm{H}), 2.20-2.13(\mathrm{~m}, 2 \mathrm{H}), 1.62-1.55(\mathrm{~m}, 4 \mathrm{H}), 1.47-1.41(\mathrm{~m}, 2 \mathrm{H}), 1.38-1.30(\mathrm{~m}, 2 \mathrm{H}), 1.22$ (s, 3H). ${ }^{13}$ C NMR: $\left(151 \mathrm{MHz}, \mathrm{CDCl}_{3}\right) \delta 173.5,151.9,139.4,120.4,117.4,106.7,41.9,36.9,29.1$, 26.2, 22.8. Rf: 0.4 (8:1 Hex:EtOAc) HRMS (m/z): Calculated for $\mathrm{C}_{13} \mathrm{H}_{16} \mathrm{~N}_{2}\left([\mathrm{M}+\mathrm{H}]^{+}\right)$: 201.1392 , found: 201.1398. IR (neat, $\mathbf{c m}^{-1}$ ): 2928, 2857, 2362, 2231, 1593, 1475, 1378, 1023, 845 .<smiles>CC1(c2ccc(C(N)=O)cn2)CCCCC1</smiles>

6-(1-Methylcyclohexyl)nicotinamide (31): Prepared according to GP1 from nicotinamide (61 mg, 0.5 mmol, 1 equiv), cesium 2-((1-methylcyclohexyl)oxy)-2-oxoacetate ${ }^{2}(191 \mathrm{mg}, 0.6 \mathrm{mmol}$, 1.2 equiv $), \operatorname{Ir}\left[\mathrm{dF}\left(\mathrm{CF}_{3}\right) \text { ppy }\right]_{2}(\mathrm{dtbbpy}) \mathrm{PF}_{6}(2.8 \mathrm{mg}, 0.0025 \mathrm{mmol}, 0.5 \mathrm{~mol} \%)$ and $\left(\mathrm{NH}_{4}\right)_{2} \mathrm{~S}_{2} \mathrm{O}_{8}(171$ $\mathrm{mg}, 0.75 \mathrm{mmol}, 1.5$ equiv) in $1 \mathrm{~mL}$ of dry DMSO. The reaction was irradiated with two $30 \mathrm{~W}$ 
Kessil blue LED lamps for $6 \mathrm{~h}$ at $30^{\circ} \mathrm{C}$. Purified by flash column chromatography (Hex $\rightarrow 1: 1$ Hex:EtOAc) to afford 31 as a colorless solid in 93\% yield (102 mg). ${ }^{20}$

${ }^{1}$ H NMR: $\left(600 \mathrm{MHz}, \mathrm{CDCl}_{3}\right) \delta 8.96(\mathrm{~d}, J=2.3 \mathrm{~Hz}, 1 \mathrm{H}), 8.09(\mathrm{dd}, J=8.3,2.4 \mathrm{~Hz}, 1 \mathrm{H}), 7.43(\mathrm{~d}$, $J=8.3 \mathrm{~Hz}, 1 \mathrm{H}), 6.09$ (b.s., 1H), 5.79 (b.s., 1H), 2.23-2.17 (m, 2H), 1.62-1.55 (m, 4H), 1.47-1.41 (m, 2H), 1.40-1.32 (m, 2H), $1.23(\mathrm{~s}, 3 \mathrm{H}) .{ }^{13} \mathbf{C}$ NMR: $\left(151 \mathrm{MHz}, \mathrm{CDCl}_{3}\right) \delta 172.8,167.8,147.6$, 135.9, 126.0, 120.3, 41.5, 37.2, 29.3, 26.3, 22.9. Rf: 0.2 (1:1 Hex:EtOAc) HRMS (m/z): Calculated for $\mathrm{C}_{13} \mathrm{H}_{18} \mathrm{~N}_{2} \mathrm{O}\left([\mathrm{M}+\mathrm{H}]^{+}\right)$: 219.1497, found: 219.1497. IR (neat, $\left.\mathbf{c m}^{-1}\right)$ : 3351, 3195 , 2856, 2360, 1662, 1599, 1404.

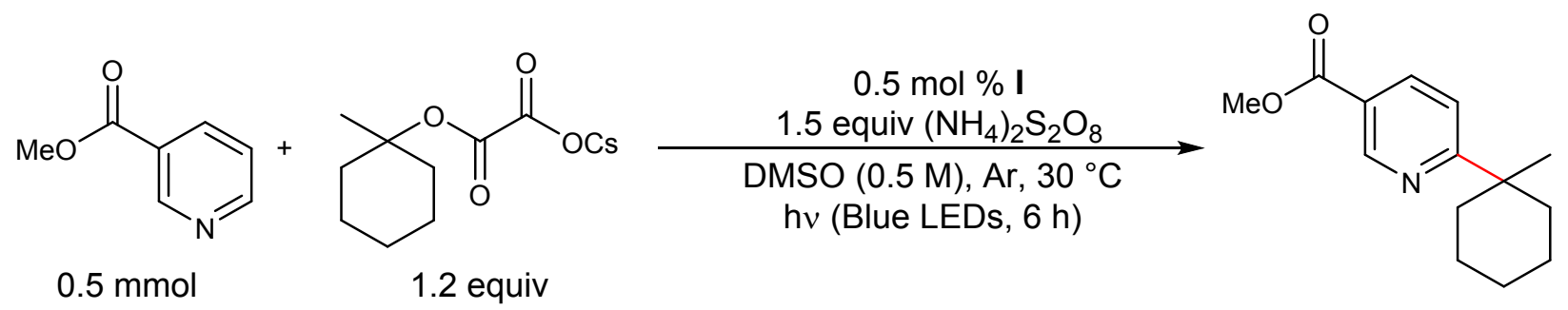

Methyl 6-(1-methylcyclohexyl)nicotinate (32): Prepared according to GP1 from methyl nicotinate (69 mg, $0.5 \mathrm{mmol}, 1$ equiv), cesium 2-((1-methylcyclohexyl)oxy)-2-oxoacetate ${ }^{2}$ (191 mg, 0.6 mmol, 1.2 equiv), $\operatorname{Ir}\left[\mathrm{dF}\left(\mathrm{CF}_{3}\right) \text { ppy }\right]_{2}(\mathrm{dtbbpy}) \mathrm{PF}_{6}(2.8 \mathrm{mg}, 0.0025 \mathrm{mmol}, 0.5 \mathrm{~mol} \%)$ and $\left(\mathrm{NH}_{4}\right)_{2} \mathrm{~S}_{2} \mathrm{O}_{8}(171 \mathrm{mg}, 0.75 \mathrm{mmol}, 1.5$ equiv) in $1 \mathrm{~mL}$ of dry DMSO. The reaction was irradiated with two $30 \mathrm{~W}$ Kessil blue LED lamps for $6 \mathrm{~h}$ at $30^{\circ} \mathrm{C}$. Purified by flash column chromatography $\left(\right.$ Hex $\rightarrow$ 9:1 Hex:EtOAc) to afford 32 as a colorless solid in 90\% yield (105 mg). ${ }^{20}$

${ }^{1}$ H NMR: $\left(600 \mathrm{MHz}, \mathrm{CDCl}_{3}\right) \delta 9.17(\mathrm{~d}, J=1.7 \mathrm{~Hz}, 1 \mathrm{H}), 8.20(\mathrm{dd}, J=8.4,2.2 \mathrm{~Hz}, 1 \mathrm{H}), 7.40(\mathrm{~d}$, $J=8.3 \mathrm{~Hz}, 1 \mathrm{H}), 3.93(\mathrm{~s}, 3 \mathrm{H}), 2.23-2.17(\mathrm{~m}, 2 \mathrm{H}), 1.61-1.55(\mathrm{~m}, 4 \mathrm{H}), 1.47-1.41(\mathrm{~m}, 2 \mathrm{H}), 1.39-1.33$ (m, 2H), $1.22(\mathrm{~s}, 3 \mathrm{H}) .{ }^{13} \mathrm{C}$ NMR: $\left(151 \mathrm{MHz}, \mathrm{CDCl}_{3}\right) \delta 173.5,166.3,150.3,137.4,123.0,119.9$ 52.3, 41.6, 37.1, 29.2, 26.3, 22.9. Rf: 0.36 (8:1 Hex:EtOAc) HRMS (m/z): Calculated for 
$\mathrm{C}_{14} \mathrm{H}_{19} \mathrm{NO}_{2}\left([\mathrm{M}+\mathrm{H}]^{+}\right): 234.1494$, found: 234.1506. IR (neat, $\left.\mathbf{~ c m}^{-1}\right):$ 2923, 2856, 2359, 1727, 1597 , $1435,1281,1116,1024,790,740$.

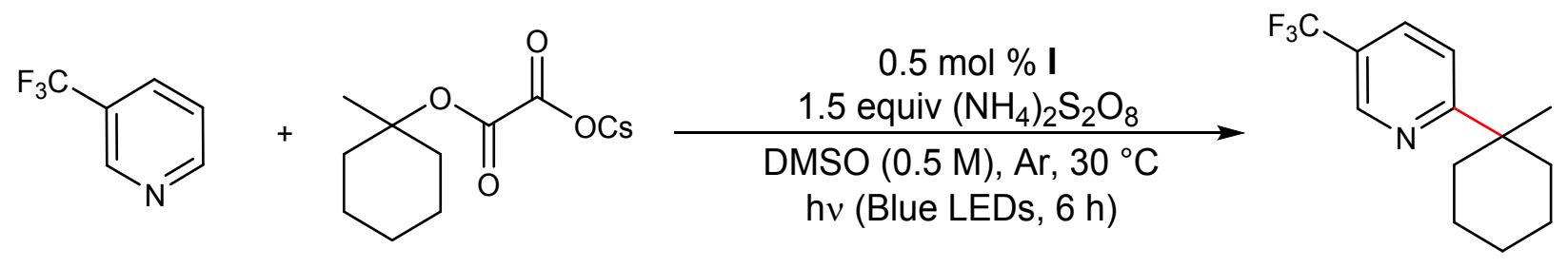

$$
0.5 \mathrm{mmol} \quad 1.2 \text { equiv }
$$

2-(1-Methylcyclohexyl)-5-(trifluoromethyl)pyridine (33): Prepared according to GP1 from 3(trifluoromethyl)pyridine (58 $\mu \mathrm{L}, 0.5 \mathrm{mmol}, 1$ equiv), cesium 2-((1-methylcyclohexyl)oxy)-2oxoacetate $^{2}$ (191 mg, $0.6 \mathrm{mmol}, 1.2$ equiv), $\operatorname{Ir}\left[\mathrm{dF}\left(\mathrm{CF}_{3}\right) \text { ppy }\right]_{2}(\mathrm{dtbbpy}) \mathrm{PF}_{6}(2.8 \mathrm{mg}, 0.0025 \mathrm{mmol}$, $0.5 \mathrm{~mol} \%)$ and $\left(\mathrm{NH}_{4}\right)_{2} \mathrm{~S}_{2} \mathrm{O}_{8}(171 \mathrm{mg}, 0.75 \mathrm{mmol}, 1.5$ equiv) in $1 \mathrm{~mL}$ of dry DMSO. The reaction was irradiated with two $30 \mathrm{~W}$ Kessil blue LED lamps for $6 \mathrm{~h}$ at $30^{\circ} \mathrm{C}$. Purified by flash column chromatography (Hex $\rightarrow$ 9:1 Hex:EtOAc) to afford $\mathbf{3 3}$ as a clear oil in 55\% yield $(67 \mathrm{mg}){ }^{20}$

${ }^{1}$ H NMR: $\left(600 \mathrm{MHz}, \mathrm{CDCl}_{3}\right) \delta 8.83(\mathrm{~s}, 1 \mathrm{H}), 7.84(\mathrm{dd}, J=8.5,2.1 \mathrm{~Hz}, 1 \mathrm{H}), 7.45(\mathrm{~d}, J=8.3 \mathrm{~Hz}, 1 \mathrm{H})$, 2.23-2.16 (m, 2H), 1.63-1.56 (m, 4H), 1.48-1.42 (m, 2H), 1.40-1.33 (m, 2H), $1.23(\mathrm{~s}, 1 \mathrm{H}) .{ }^{13} \mathrm{C}$ NMR: $\left(151 \mathrm{MHz}, \mathrm{CDCl}_{3}\right) \delta 172.9,145.9$ (q, $\left.J=4.1 \mathrm{~Hz}\right), 133.4$ (q, $\left.J=3.4 \mathrm{~Hz}\right), 123.5$ (q, $\left.J=33 \mathrm{~Hz}\right)$, 124.0 (q, $J=272 \mathrm{~Hz}), 120.0,41.5,37.1,29.3,26.3,22.8$. Rf: 0.74 (8:1 Hex:EtOAc) HRMS (m/z): Calculated for $\mathrm{C}_{13} \mathrm{H}_{16} \mathrm{~F}_{3} \mathrm{~N}\left([\mathrm{M}+\mathrm{H}]^{+}\right)$: 244.1313, found: 244.1307. IR (neat, $\left.\mathbf{c m}^{-1}\right)$ : 2923, 2359, 2341, 1054, 1033, 1014. 


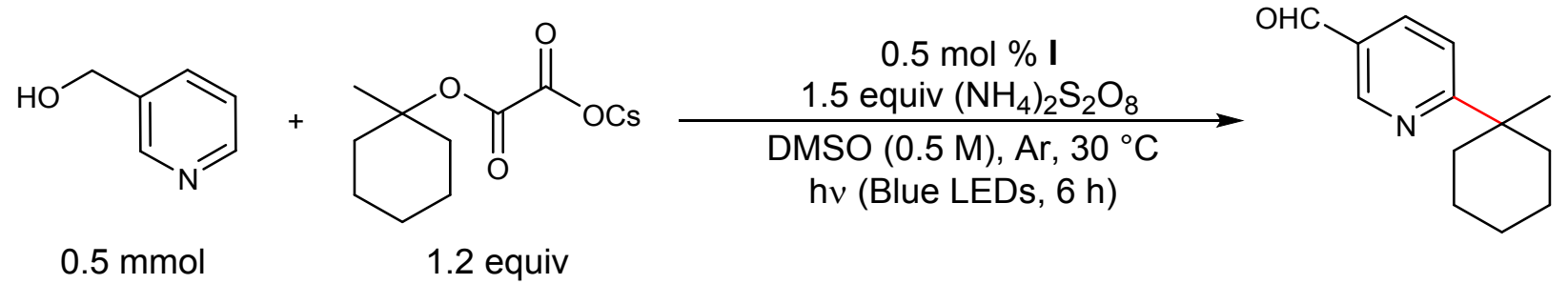

6-(1-Methylcyclohexyl)nicotinaldehyde (34): Prepared according to GP1 from 3pyridinemethanol (49 $\mu \mathrm{L}, 0.5 \mathrm{mmol}, 1$ equiv), cesium 2-((1-methylcyclohexyl)oxy)-2-oxoacetate ${ }^{2}$ (191 mg, $0.6 \mathrm{mmol}, 1.2$ equiv), $\operatorname{Ir}\left[\mathrm{dF}\left(\mathrm{CF}_{3}\right) \text { ppy }\right]_{2}(\mathrm{dtbbpy}) \mathrm{PF}_{6}(2.8 \mathrm{mg}, 0.0025 \mathrm{mmol}, 0.5 \mathrm{~mol} \%)$ and $\left(\mathrm{NH}_{4}\right)_{2} \mathrm{~S}_{2} \mathrm{O}_{8}(171 \mathrm{mg}, 0.75 \mathrm{mmol}, 1.5$ equiv $)$ in $1 \mathrm{~mL}$ of dry DMSO. The reaction was irradiated with two $30 \mathrm{~W}$ Kessil blue LED lamps for $6 \mathrm{~h}$ at $30^{\circ} \mathrm{C}$. Purified by flash column chromatography $\left(\right.$ Hex $\rightarrow$ 9:1 Hex:EtOAc) to afford 34 as a clear oil in $60 \%$ yield $(61 \mathrm{mg}) .{ }^{20}$

${ }^{1}$ H NMR: $\left(600 \mathrm{MHz}, \mathrm{CDCl}_{3}\right) \delta 10.08(\mathrm{~s}, 1 \mathrm{H}), 9.02(\mathrm{~d}, J=2.0 \mathrm{~Hz}, 1 \mathrm{H}), 8.10(\mathrm{dd}, J=8.3,2.2 \mathrm{~Hz}$, $1 \mathrm{H}), 7.51(\mathrm{~d}, J=8.3 \mathrm{~Hz}, 1 \mathrm{H}), 2.26-2.16(\mathrm{~m}, 2 \mathrm{H}), 1.64-1.56(\mathrm{~m}, 4 \mathrm{H}), 1.49-1.42(\mathrm{~m}, 2 \mathrm{H}), 1.41-1.33$ (m, 2H), 1.24 (s, 3H). ${ }^{13}$ C NMR: (151 MHz, $\left.\mathrm{CDCl}_{3}\right) \delta 190.9,175.3,151.9,136.0,129.0,120.8$, 42.0, 37.1, 29.2, 26.3, 22.9. Rf: 0.35 (8:1 Hex:EtOAc) HRMS (m/z): Calculated for $\mathrm{C}_{13} \mathrm{H}_{17} \mathrm{NO}$ ([M+H] $\left.]^{+}\right):$204.1388, found: 204.1389. IR (neat, $\left.\mathbf{c m}^{-1}\right):$ 2927, 2857, 2359, 1705, 1594, 1559, 1456, 1361, 1088, 840.<smiles>c1ccc2nccnc2c1</smiles>

$0.5 \mathrm{mmol}$<smiles>COC(=O)C(=O)OC1(C)CCCCC1</smiles>

1.2 equiv

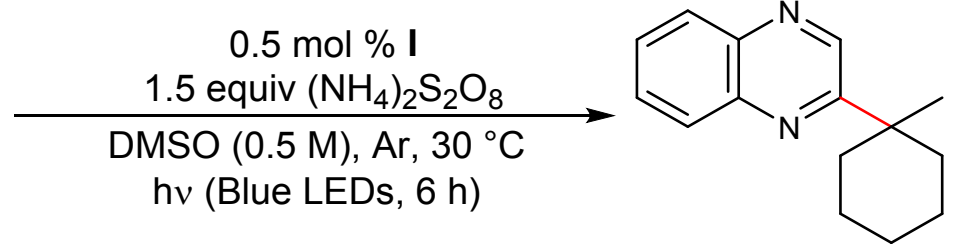
hv (Blue LEDs, $6 \mathrm{~h}$ )

2-(1-Methylcyclohexyl)quinoxaline (35): Prepared according to GP1 from quinoxaline (65 mg, $0.5 \mathrm{mmol}, 1$ equiv), cesium 2-((1-methylcyclohexyl)oxy)-2-oxoacetate ${ }^{2}(191 \mathrm{mg}, 0.6 \mathrm{mmol}, 1.2$ equiv), $\operatorname{Ir}\left[\mathrm{dF}\left(\mathrm{CF}_{3}\right) \mathrm{ppy}\right]_{2}(\mathrm{dtbbpy}) \mathrm{PF}_{6}(2.8 \mathrm{mg}, 0.0025 \mathrm{mmol}, 0.5 \mathrm{~mol} \%)$ and $\left(\mathrm{NH}_{4}\right)_{2} \mathrm{~S}_{2} \mathrm{O}_{8}(171 \mathrm{mg}$, 
$0.75 \mathrm{mmol}, 1.5$ equiv) in $1 \mathrm{~mL}$ of dry DMSO. The reaction was irradiated with two $30 \mathrm{~W}$ Kessil blue LED lamps for $6 \mathrm{~h}$ at $30{ }^{\circ} \mathrm{C}$. Purified by flash column chromatography (Hex $\rightarrow$ 9:1 Hex:EtOAc) to afford $\mathbf{3 5}$ as a clear oil in $54 \%$ yield $(61 \mathrm{mg})$.

${ }^{1}$ H NMR: $\left(600 \mathrm{MHz}, \mathrm{CDCl}_{3}\right) \delta 8.94(\mathrm{~s}, 1 \mathrm{H}), 8.07-8.04(\mathrm{~m}, 2 \mathrm{H})$, 7.74-7.67 (m, 2H), 2.42-2.35 (m, 2H), 1.71-1.60 (m, 4H), 1.50-1.42 (m, 4H), 1.36 (s, 3H). ${ }^{13} \mathrm{C}$ NMR: $\left(151 \mathrm{MHz}, \mathrm{CDCl}_{3}\right) \delta 163.3$, $144.0,142.1,140.7,129.7,129.4,129.04,128.97,40.8,36.9,29.0,26.3,22.8$. Rf: $0.14(8: 1$ Hex:EtOAc) HRMS (m/z): Calculated for $\mathrm{C}_{15} \mathrm{H}_{18} \mathrm{~N}_{2}\left([\mathrm{M}+\mathrm{H}]^{+}\right)$: 227.1548, found: 227.1544. IR (neat, $\mathbf{c m}^{-1}$ ): 2926, 2854, 2361, 1556, 1489, 1455, 1093, 960, 760 .

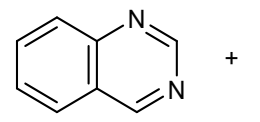

$0.5 \mathrm{mmol}$<smiles>COC(=O)C(=O)OC1(C)CCCCC1</smiles>

1.2 equiv

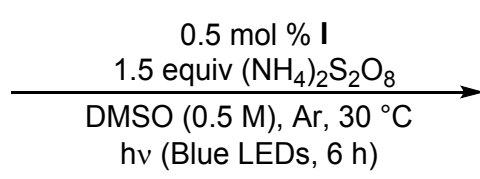
hv (Blue LEDs, $6 \mathrm{~h}$ )

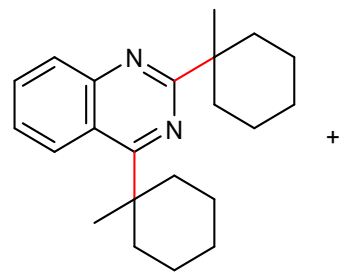

36

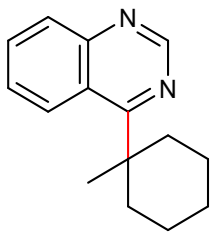

\section{Reaction of quinazoline to form a mixture of 2,4-di-(1-methylcyclohexyl)quinazoline (36) and}

4-(1-methylcyclohexyl)quinazoline: Prepared according to GP1 from quinazoline (65 mg, 0.5 mmol, 1 equiv), cesium 2-((1-methylcyclohexyl)oxy)-2-oxoacetate ${ }^{2}$ (191 mg, $0.6 \mathrm{mmol}, 1.2$ equiv), $\operatorname{Ir}\left[\mathrm{dF}\left(\mathrm{CF}_{3}\right) \text { ppy }\right]_{2}(\mathrm{dtbbpy}) \mathrm{PF}_{6}(2.8 \mathrm{mg}, 0.0025 \mathrm{mmol}, 0.5 \mathrm{~mol} \%)$ and $\left(\mathrm{NH}_{4}\right)_{2} \mathrm{~S}_{2} \mathrm{O}_{8}(171 \mathrm{mg}$, $0.75 \mathrm{mmol}, 1.5$ equiv) in $1 \mathrm{~mL}$ of dry DMSO. The reaction was irradiated with two $30 \mathrm{~W}$ Kessil blue LED lamps for $6 \mathrm{~h}$ at $30{ }^{\circ} \mathrm{C}$. Purified by flash column chromatography (Hex $\rightarrow$ 9:1 Hex:EtOAc) to afford:

2,4-di-(1-methylcyclohexyl)quinazoline (36) as a clear oil in 33\% yield (32 mg).

${ }^{1}$ H NMR: $\left(600 \mathrm{MHz}, \mathrm{CDCl}_{3}\right) \delta 8.41(\mathrm{~d}, J=8.6 \mathrm{~Hz}, 1 \mathrm{H}), 8.01(\mathrm{~d}, J=8.3 \mathrm{~Hz}, 1 \mathrm{H}), 7.75-7.71(\mathrm{~m}, 1 \mathrm{H})$, 7.47-7.43 (m, 1H), 2.59-2.48 (m, 4H), 1.80-1.74 (m, 2H), 1.65-1.55 (m, 6H), $1.59(\mathrm{~s}, 3 \mathrm{H}), 1.54-$ 
$1.38(\mathrm{~m}, 8 \mathrm{H}), 1.34(\mathrm{~s}, 3 \mathrm{H}) .{ }^{13} \mathrm{C}$ NMR: $\left(151 \mathrm{MHz}, \mathrm{CDCl}_{3}\right) \delta 175.2,170.1,152.0,131.6,130.3$, $126.2,124.9,121.1,44.1,43.2,38.7,36.9,28.8,28.2,26.6,26.5,23.3,23.2$. Rf: $0.52(8: 1$ Hex:EtOAc) HRMS (m/z): Calculated for $\mathrm{C}_{22} \mathrm{H}_{30} \mathrm{~N}_{2}\left([\mathrm{M}+\mathrm{H}]^{+}\right)$: 323.2487 , found: 323.2478. IR (neat, $\mathbf{c m}^{-1}$ ): 2924, 2852, 2359, 1548, 1493, 1447, 764 .

4-(1-methylcyclohexyl)quinazoline as a clear oil in 9\% yield from quinazoline (14 mg).

${ }^{1}$ H NMR: $\left(600 \mathrm{MHz}, \mathrm{CDCl}_{3}\right) \delta 9.37(\mathrm{~s}, 1 \mathrm{H}), 7.99(\mathrm{~d}, J=8.4 \mathrm{~Hz}, 1 \mathrm{H}), 7.89-7.84(\mathrm{~m}, 4 \mathrm{H}), 7.59-7.56$ (m, 1H), 2.54-2.47 (m, 2H), 1.65-1.58 (m, 4H), 1.49-1.38 (m, 4H), $1.36(\mathrm{~s}, 3 \mathrm{H}) .{ }^{13} \mathbf{C}$ NMR: $(151$ $\left.\mathrm{MHz}, \mathrm{CDCl}_{3}\right) \delta 172.9,160.2,150.5,133.6,128.5,127.0,126.9,122.9,43.2,37.1,28.9,26.4,23.2$. Rf: 0.39 (8:1 Hex:EtOAc) HRMS (m/z): Calculated for $\mathrm{C}_{15} \mathrm{H}_{18} \mathrm{~N}_{2}\left([\mathrm{M}+\mathrm{H}]^{+}\right)$: 227.1548, found: 227.1554. IR (neat, $\mathbf{c m}^{-1}$ ): 2924, 2852, 2360, 2340, 1620, 1557, 1447, 760.

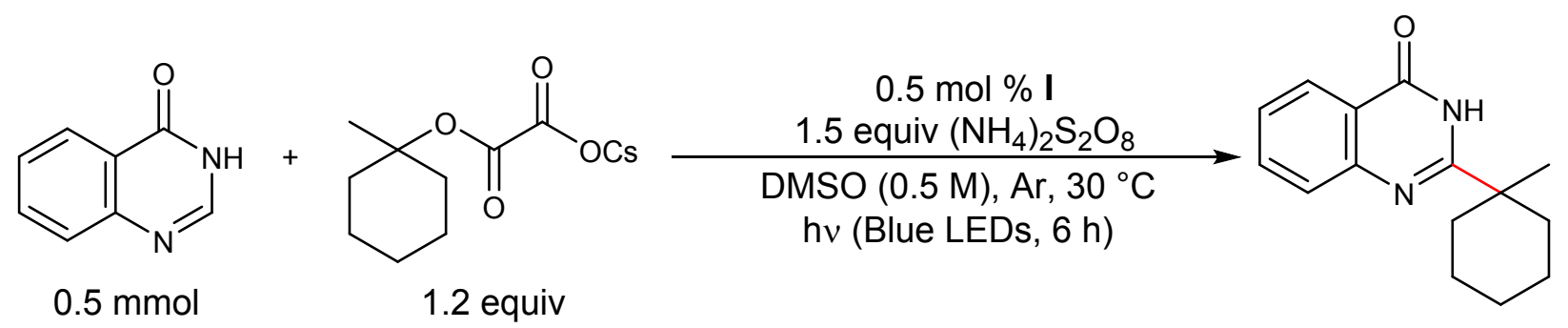

2-(1-Methylcyclohexyl)quinazolin-4(3H)-one (37): Prepared according to GP1 from Quinazolin-4(3H)-one (73 mg, $0.5 \mathrm{mmol}, 1$ equiv), cesium 2-((1-methylcyclohexyl)oxy)-2oxoacetate $^{2}$ (191 mg, $0.6 \mathrm{mmol}, 1.2$ equiv), $\operatorname{Ir}\left[\mathrm{dF}\left(\mathrm{CF}_{3}\right) \text { ppy }\right]_{2}(\mathrm{dtbbpy}) \mathrm{PF}_{6}(2.8 \mathrm{mg}, 0.0025 \mathrm{mmol}$, $0.5 \mathrm{~mol} \%)$ and $\left(\mathrm{NH}_{4}\right)_{2} \mathrm{~S}_{2} \mathrm{O}_{8}(171 \mathrm{mg}, 0.75 \mathrm{mmol}, 1.5$ equiv) in $1 \mathrm{~mL}$ of dry DMSO. The reaction was irradiated with two $30 \mathrm{~W}$ Kessil blue LED lamps for $6 \mathrm{~h}$ at $30^{\circ} \mathrm{C}$. EtOAc was used in place of $\mathrm{Et}_{2} \mathrm{O}$ during the work-up. Purified by flash column chromatography (Hex $\rightarrow$ 85:15 Hex:EtOAc) to afford $\mathbf{3 7}$ as a colorless solid in 51\% yield (62 $\mathrm{mg})$. 
1H NMR: (600 MHz, $\left.\mathrm{CDCl}_{3}\right) \delta 10.14$ (b.s., $\left.1 \mathrm{H}\right), 8.27$ (d, $\left.J=7.9 \mathrm{~Hz}, 1 \mathrm{H}\right), 7.77-7.70$ (m, 2H), 7.47$7.43(\mathrm{~m}, 1 \mathrm{H}), 2.29-2.22(\mathrm{~m}, 2 \mathrm{H}), 1.67-1.41(\mathrm{~m}, 8 \mathrm{H}), 1.36(\mathrm{~s}, 3 \mathrm{H}) .{ }^{13} \mathbf{C}$ NMR: $\left(151 \mathrm{MHz}, \mathrm{CDCl}_{3}\right)$ $\delta 163.5,161.1,149.4,134.6,127.8,126.5,126.3,120.8,41.1,36.0,27.2,25.9,22.7$. Rf: 0.48 (8:1 Hex:EtOAc) HRMS (m/z): Calculated for $\mathrm{C}_{15} \mathrm{H}_{18} \mathrm{~N}_{2} \mathrm{O}\left([\mathrm{M}+\mathrm{Na}]^{+}\right)$: 265.1317, found: 265.1317. IR (neat, $\mathbf{c m}^{-1}$ ): 3183, 3068, 2925, 2852, 2362, 1667, 1607, 1470, 772 .

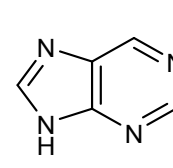

$0.5 \mathrm{mmol}$<smiles>COC(=O)C(=O)OC1(C)CCCCC1</smiles>

1.2 equiv

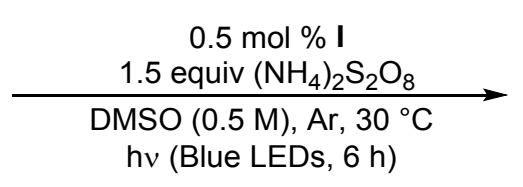

hv (Blue LEDs, $6 \mathrm{~h}$ )

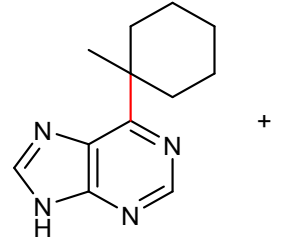

38

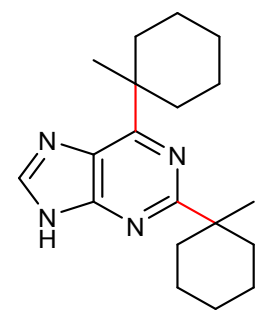

6-(1-Methylcyclohexyl)-9H-purine (38): Prepared according to GP1 from purine (60 mg, 0.5 mmol, 1 equiv), cesium 2-((1-methylcyclohexyl)oxy)-2-oxoacetate ${ }^{2}$ (191 mg, $0.6 \mathrm{mmol}, 1.2$ equiv), $\operatorname{Ir}\left[\mathrm{dF}\left(\mathrm{CF}_{3}\right) \mathrm{ppy}\right]_{2}(\mathrm{dtbbpy}) \mathrm{PF}_{6}(2.8 \mathrm{mg}, 0.0025 \mathrm{mmol}, 0.5 \mathrm{~mol} \%)$ and $\left(\mathrm{NH}_{4}\right)_{2} \mathrm{~S}_{2} \mathrm{O}_{8}(171 \mathrm{mg}$, $0.75 \mathrm{mmol}, 1.5$ equiv) in $1 \mathrm{~mL}$ of dry DMSO. The reaction was irradiated with two $30 \mathrm{~W}$ Kessil blue LED lamps for $6 \mathrm{~h}$ at $30^{\circ} \mathrm{C}$. EtOAc was used in place of $\mathrm{Et}_{2} \mathrm{O}$ during the work-up. Purified by flash column chromatography (DCM $\rightarrow$ 99:1 DCM:MeOH) to afford:

6-(1-methylcyclohexyl)-9H-purine 38 as a colorless solid in $74 \%$ yield $(80 \mathrm{mg})$.

${ }^{1}$ H NMR: $\left(600 \mathrm{MHz}, \mathrm{CD}_{3} \mathrm{OD}\right) \delta 8.82(\mathrm{~s}, 1 \mathrm{H}), 8.38(\mathrm{~s}, 1 \mathrm{H}), 2.74-2.61(\mathrm{~m}, 2 \mathrm{H}), 1.71-1.60(\mathrm{~m}, 4 \mathrm{H})$, 1.53-1.44 (m, 2H), 1.44-1.35 (m, 2H), $1.42(\mathrm{~s}, 3 \mathrm{H}) .{ }^{13}$ C NMR: (151 MHz, $\left.\mathrm{CD}_{3} \mathrm{OD}\right) \delta 167.9,152.6$, 144.6, 43.2, 37.6, 28.4, 27.1, 24.1. Rf: 0.33 (9:1 DCM:MeOH) HRMS (m/z): Calculated for $\mathrm{C}_{12} \mathrm{H}_{16} \mathrm{~N}_{4}\left([\mathrm{M}+\mathrm{H}]^{+}\right): 217.1453$, found: 217.1450. IR (neat, $\left.\mathbf{c m}^{-1}\right): 3102,2929,2854,2362,1570$, 1464, 1324, 1235, 924, 645. 
2,6-Di-(1-methylcyclohexyl)-9H-purine was isolated also as a colorless solid in $13 \%$ yield from purine (20 mg):

${ }^{1}$ H NMR: (600 MHz, Acetone-d $\left.{ }_{6}\right) \delta 11.94$ (b.s., 1H), 8.68 (s, 1H), 2.97-2.91 (m, 2H), 2.44-2.37 (m, 2H), 1.66-1.46 (m, 12H), 1.42-1.34 (m, 2H), $1.40(\mathrm{~s}, 3 \mathrm{H}), 1.38(\mathrm{~s}, 3 \mathrm{H}) .{ }^{13} \mathbf{C}$ NMR: $(151 \mathrm{MHz}$ Acetone-d $\left.{ }_{6}\right) \delta 166.4,161.9,154.8,151.7,132.4,42.9,38.5,37.5,37.4,29.3,27.0,26.6,24.1,23.6$. Rf: 0.47 (9:1 DCM:MeOH) HRMS (m/z): Calculated for $\mathrm{C}_{19} \mathrm{H}_{28} \mathrm{~N}_{4}\left([\mathrm{M}+\mathrm{H}]^{+}\right)$: 313.2392, found: 313.2388. IR (neat, $\mathbf{~ c m}^{-1}$ ): 3087, 2927, 2854, 2359, 1598, 1582, 1448, 1418, 1326.

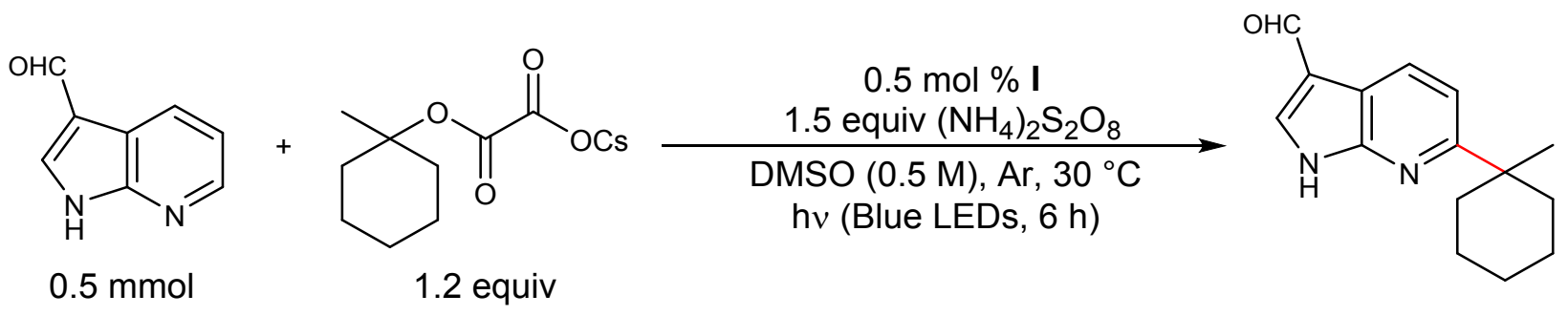

6-(1-Methylcyclohexyl)-1H-pyrrolo[2,3-b]pyridine-3-carbaldehyde (39): Prepared according to GP1 from 7-azaindole-3-carboxaldehyde (58 mg, $0.4 \mathrm{mmol}, 1$ equiv), cesium 2-((1methylcyclohexyl)oxy)-2-oxoacetate ${ }^{2}\left(191 \mathrm{mg}, 0.6 \mathrm{mmol}, 1.5\right.$ equiv), $\operatorname{Ir}\left[\mathrm{dF}\left(\mathrm{CF}_{3}\right) \mathrm{ppy}\right]_{2}(\mathrm{dtbbpy}) \mathrm{PF}_{6}$ (2.8 mg, $0.0025 \mathrm{mmol}, 0.625 \mathrm{~mol} \%$ ) and $\left(\mathrm{NH}_{4}\right)_{2} \mathrm{~S}_{2} \mathrm{O}_{8}(171 \mathrm{mg}, 0.75 \mathrm{mmol}, 1.875$ equiv) in $1 \mathrm{~mL}$ of dry DMSO. The reaction was irradiated with two $30 \mathrm{~W}$ Kessil blue LED lamps for $6 \mathrm{~h}$ at $30^{\circ} \mathrm{C}$. EtOAc was used in place of $\mathrm{Et}_{2} \mathrm{O}$ during the work-up. Purified by flash column chromatography $\left(\mathrm{Hex} \rightarrow 7: 3\right.$ EtOAc:Hex) to afford $\mathbf{3 9}$ as a pale brown solid in $32 \%$ yield $(31 \mathrm{mg}){ }^{20}$

1'H NMR: (600 MHz, $\left.\mathrm{CDCl}_{3}\right) \delta 10.69$ (b.s., $\left.1 \mathrm{H}\right), 10.57$ (s, $\left.1 \mathrm{H}\right), 8.70(\mathrm{~d}, J=7.7 \mathrm{~Hz}, 1 \mathrm{H}), 8.34$ (b.s., 1H), 2.29-2.22 (m, 2H), 1.96-1.89 (m, 2H), 1.75-1.67 (m, 2H), 1.62-1.54 (m, 3H), $1.60(\mathrm{~s}, 3 \mathrm{H})$, 1.53-1.45 (m, 1H). ${ }^{13} \mathrm{C}$ NMR: (151 MHz, $\left.\mathrm{CDCl}_{3}\right) \delta$ 186.3, 158.3, 147.5, 143.9, 131.2, 120.6, 119.0, 112.9, 39.1, 38.7, 29.1, 25.8, 22.6. Rf: 0.15 (2:1 Hex:EtOAc) HRMS (m/z): Calculated for 
$\mathrm{C}_{15} \mathrm{H}_{18} \mathrm{~N}_{2} \mathrm{O}\left([\mathrm{M}+\mathrm{H}]^{+}\right): 243.1497$, found: 243.1505. IR (neat, $\left.\mathbf{c m}^{-1}\right): 3161,2930,2859,2360,2338$, $1647,1586,1437,1388,1276,776$.

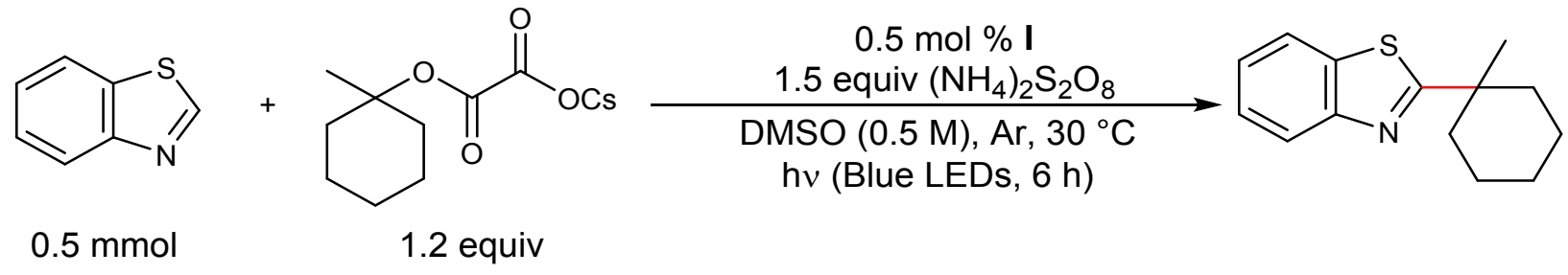

2-(1-Methylcyclohexyl)benzo[d]thiazole (40): Prepared according to GP1 from benzothiazole (55 $\mu \mathrm{L}, 0.5 \mathrm{mmol}, 1$ equiv), cesium 2-((1-methylcyclohexyl)oxy)-2-oxoacetate ${ }^{2}$ (191 mg, 0.6 mmol, 1.2 equiv), $\operatorname{Ir}\left[\mathrm{dF}\left(\mathrm{CF}_{3}\right) \text { ppy }\right]_{2}(\mathrm{dtbbpy}) \mathrm{PF}_{6}(2.8 \mathrm{mg}, 0.0025 \mathrm{mmol}, 0.5 \mathrm{~mol} \%)$ and $\left(\mathrm{NH}_{4}\right)_{2} \mathrm{~S}_{2} \mathrm{O}_{8}$ (171 mg, $0.75 \mathrm{mmol}, 1.5$ equiv) in $1 \mathrm{~mL}$ of dry DMSO. The reaction was irradiated with two 30 W Kessil blue LED lamps for $6 \mathrm{~h}$ at $30^{\circ} \mathrm{C}$. Purified by flash column chromatography (Hex $\rightarrow$ 9:1 Hex:EtOAc) to afford $\mathbf{4 0}$ as a clear oil in $52 \%$ yield $(60 \mathrm{mg})$.

${ }^{1}$ H NMR: $\left(600 \mathrm{MHz}, \mathrm{CDCl}_{3}\right) \delta 7.99(\mathrm{~d}, \mathrm{~J}=8.2 \mathrm{~Hz}, 1 \mathrm{H}), 7.86(\mathrm{~d}, J=7.9 \mathrm{~Hz}, 1 \mathrm{H}), 7.46-7.42(\mathrm{~m}, 1 \mathrm{H})$, 7.35-7.32 (m, 1H), 2.27-2.21 (m, 2H), 1.74-1.68 (m, 2H), 1.68-1.61 (m, 2H), 1.60-1.53 (m, 2H), 1.53-1.43 (m, 2H), $1.41(\mathrm{~s}, 3 \mathrm{H}) .{ }^{13} \mathrm{C}$ NMR: $\left(151 \mathrm{MHz}, \mathrm{CDCl}_{3}\right) \delta 181.8,153.4,135.1,125.7,124.6$, 122.8, 121.6, 42.2, 38.6, 29.9, 25.9, 22.8. Rf: 0.58 (8:1 Hex:EtOAc) HRMS (m/z): Calculated for $\mathrm{C}_{14} \mathrm{H}_{17} \mathrm{NS}\left([\mathrm{M}+\mathrm{H}]^{+}\right): 232.1160$, found: 232.1165. IR (neat, $\left.\mathbf{c m}^{-1}\right)$ : 2925, 2852, 1508, 1437, 1009, $757,729$.

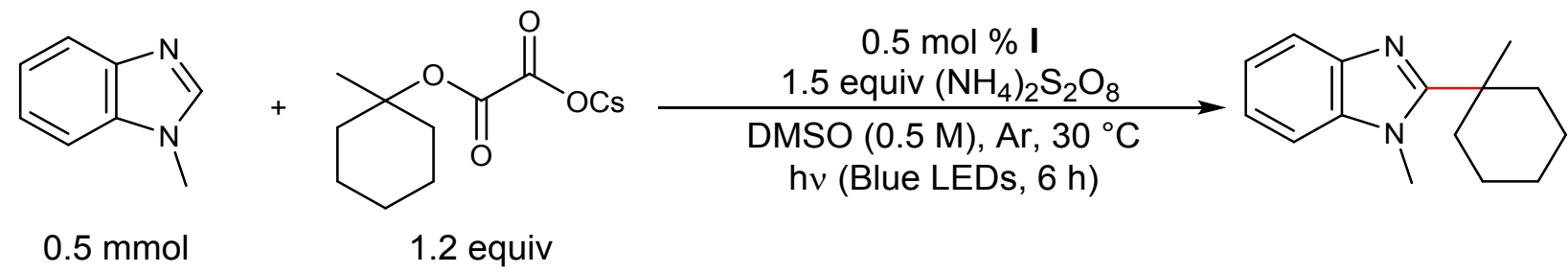

1-Methyl-2-(1-methylcyclohexyl)-1 $H$-benzo $[\boldsymbol{d}]$ imidazole (41): Prepared according to GP1 from 1-methylbenzimidazole (66 mg, $0.5 \mathrm{mmol}, 1$ equiv), cesium 2-((1-methylcyclohexyl)oxy)-2- 
oxoacetate $^{2}\left(191 \mathrm{mg}, 0.6 \mathrm{mmol}, 1.2\right.$ equiv), $\operatorname{Ir}\left[\mathrm{dF}\left(\mathrm{CF}_{3}\right) \mathrm{ppy}\right]_{2}(\mathrm{dtbbpy}) \mathrm{PF}_{6}(2.8 \mathrm{mg}, 0.0025 \mathrm{mmol}$, $0.5 \mathrm{~mol} \%)$ and $\left(\mathrm{NH}_{4}\right)_{2} \mathrm{~S}_{2} \mathrm{O}_{8}(171 \mathrm{mg}, 0.75 \mathrm{mmol}, 1.5$ equiv) in $1 \mathrm{~mL}$ of dry DMSO. The reaction was irradiated with two $30 \mathrm{~W}$ Kessil blue LED lamps for $6 \mathrm{~h}$ at $30^{\circ} \mathrm{C}$. Purified by flash column chromatography (Hex $\rightarrow$ 9:1 Hex:EtOAc) to afford $\mathbf{4 1}$ as a clear oil in 37\% yield (42 mg).

${ }^{1}$ H NMR: $\left(600 \mathrm{MHz}, \mathrm{CDCl}_{3}\right) \delta$ 7.79-7.75 (m, 1H), 7.32-7.20 (m, 3H), $3.90(\mathrm{~s}, 3 \mathrm{H})$, 2.41-2.34 (m, 2H), 1.77-1.71 (m, 2H), 1.66-1.57 (m, 4H), 1.53-1.45 (m, 2H), 1.44 (s, 3H). ${ }^{13}$ C NMR: (151 MHz, $\left.\mathrm{CDCl}_{3}\right) \delta 160.6,141.9,137.3,122.1,121.7,119.5,108.8,37.9,37.3,32.6,26.1,25.5,22.7 . \mathbf{R f}:$ 0.14 (8:1 Hex:EtOAc) HRMS (m/z): Calculated for $\mathrm{C}_{15} \mathrm{H}_{20} \mathrm{~N}_{2}\left([\mathrm{M}+\mathrm{H}]^{+}\right)$: 229.1705, found: 229.1694. IR (neat, $\left.\mathbf{c m}^{-1}\right):$ 2927, 2853, 2359, 1465, 1368, 1322, 1286, 745.

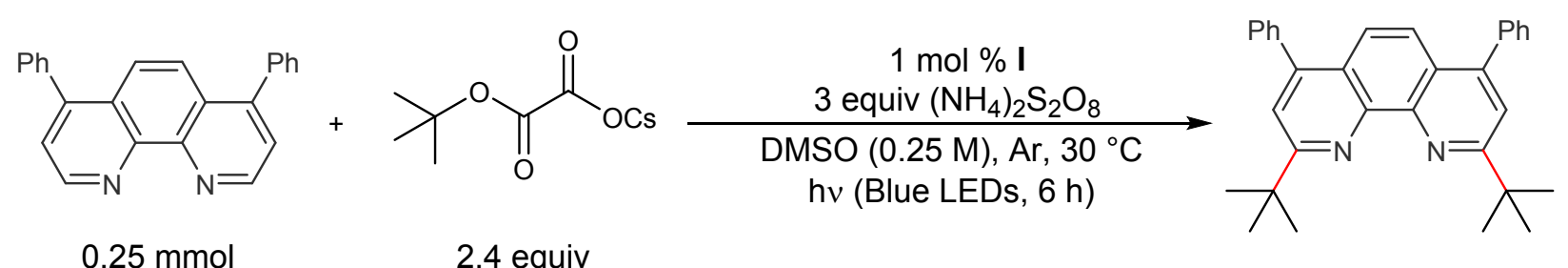

2,9-Di-tert-butyl-4,7-diphenyl-1,10-phenanthroline (42): Prepared according to GP1 from bathophenanthroline ( $83 \mathrm{mg}, 0.25 \mathrm{mmol}, 1$ equiv), cesium 2-(tert-butoxy)-2-oxoacetate ${ }^{2}$ (167 mg, $0.6 \mathrm{mmol}, 2.4$ equiv), $\operatorname{Ir}\left[\mathrm{dF}\left(\mathrm{CF}_{3}\right) \mathrm{ppy}\right]_{2}(\mathrm{dtbbpy}) \mathrm{PF}_{6}(2.8 \mathrm{mg}, 0.0025 \mathrm{mmol}, 1 \mathrm{~mol} \%)$ and $\left(\mathrm{NH}_{4}\right)_{2} \mathrm{~S}_{2} \mathrm{O}_{8}(171 \mathrm{mg}, 0.75 \mathrm{mmol}, 3$ equiv) in $1 \mathrm{~mL}$ of dry DMSO. The reaction was irradiated with two $30 \mathrm{~W}$ Kessil blue LED lamps for $6 \mathrm{~h}$ at $30{ }^{\circ} \mathrm{C}$. DCM was used in place of $\mathrm{Et}_{2} \mathrm{O}$ during the work-up. Purified by flash column chromatography (Hex $\rightarrow$ 9:1 Hex:EtOAc) to afford $\mathbf{4 2}$ as a pale yellow solid in $55 \%$ yield $(61 \mathrm{mg})$.

${ }^{1}$ H NMR: $\left(600 \mathrm{MHz}, \mathrm{CDCl}_{3}\right) \delta 7.73(\mathrm{~s}, 2 \mathrm{H}), 7.64(\mathrm{~s}, 2 \mathrm{H}), 7.53-7.50(\mathrm{~m}, 8 \mathrm{H}), 7.49-7.46(\mathrm{~m}, 2 \mathrm{H})$, 1.64 (s, 18H). ${ }^{13} \mathrm{C}$ NMR: $\left(151 \mathrm{MHz}, \mathrm{CDCl}_{3}\right) \delta$ 168.8, 148.4, 145.6, 139.2, 129.9, 128.6, 128.2, 124.9, 123.1, 120.1, 38.9, 30.5. Rf: 0.50 (8:1 Hex:EtOAc). Reference: Chem. Sci. 2017, 8, 3512. 


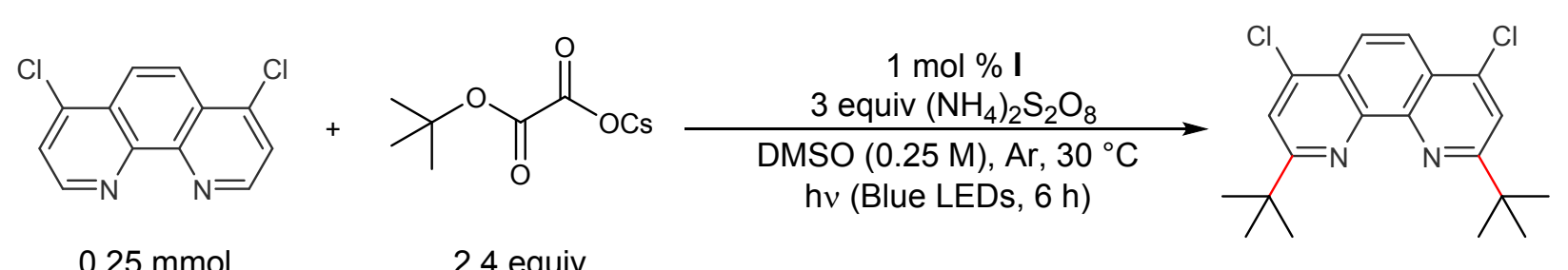

$0.25 \mathrm{mmol}$

2.4 equiv

2,9-Di-tert-butyl-4,7-dichloro-1,10-phenanthroline (43): Prepared according to GP1 from 4,7dichloro-1,10-phenanthroline (62 mg, $0.25 \mathrm{mmol}, 1$ equiv), cesium 2-(tert-butoxy)-2-oxoacetate ${ }^{2}$ (167 mg, $0.6 \mathrm{mmol}, 2.4$ equiv), $\operatorname{Ir}\left[\mathrm{dF}\left(\mathrm{CF}_{3}\right) \text { ppy }\right]_{2}\left(\mathrm{dtbbpy} \mathrm{PF}_{6}(2.8 \mathrm{mg}, 0.0025 \mathrm{mmol}, 1 \mathrm{~mol} \%)\right.$ and $\left(\mathrm{NH}_{4}\right)_{2} \mathrm{~S}_{2} \mathrm{O}_{8}(171 \mathrm{mg}, 0.75 \mathrm{mmol}, 3$ equiv) in $1 \mathrm{~mL}$ of dry DMSO. The reaction was irradiated with two $30 \mathrm{~W}$ Kessil blue LED lamps for $6 \mathrm{~h}$ at $30{ }^{\circ} \mathrm{C}$. DCM was used in place of $\mathrm{Et}_{2} \mathrm{O}$ during the work-up. Purified by flash column chromatography (Hex $\rightarrow$ 9:1 Hex:EtOAc) to afford 43 as a colorless solid in 38\% yield (34 mg).

${ }^{1} \mathbf{H}$ NMR: $\left(600 \mathrm{MHz}, \mathrm{CDCl}_{3}\right) \delta 8.22(\mathrm{~s}, 2 \mathrm{H}), 7.80(\mathrm{~s}, 2 \mathrm{H}), 1.57$ (s, 18H). ${ }^{13} \mathrm{C}$ NMR: $(151 \mathrm{MHz}$, $\left.\mathrm{CDCl}_{3}\right) \delta 170.0,145.9,142.6,125.1,122.3,120.6,39.0,30.2$. Rf: 0.58 (8:1 Hex:EtOAc) HRMS (m/z): Calculated for $\mathrm{C}_{20} \mathrm{H}_{22} \mathrm{Cl}_{2} \mathrm{~N}_{2}\left([\mathrm{M}+\mathrm{H}]^{+}\right): 361.1238$, found: 361.1249. IR (neat, $\left.\mathbf{c m}^{-1}\right):$ 2960, 2867, 2359, 2342, 1577, 1479, 1362, 1139, 866, 845, 814, 796.

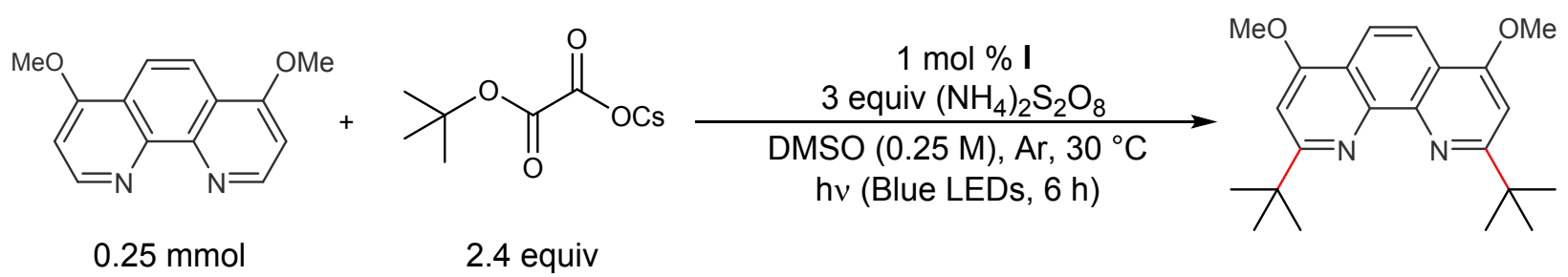

2,9-Di-tert-butyl-4,7-dimethoxy-1,10-phenanthroline (44): Prepared according to GP1 from 4,7-dimethoxy-1,10-phenanthroline (60 mg, $0.25 \mathrm{mmol}, 1$ equiv), cesium 2-(tert-butoxy)-2oxoacetate $^{2}$ (167 mg, 0.6 mmol, 2.4 equiv), $\operatorname{Ir}\left[\mathrm{dF}\left(\mathrm{CF}_{3}\right) \text { ppy }\right]_{2}(\mathrm{dtbbpy}) \mathrm{PF}_{6}(2.8 \mathrm{mg}, 0.0025 \mathrm{mmol}, 1$ mol \%) and $\left(\mathrm{NH}_{4}\right)_{2} \mathrm{~S}_{2} \mathrm{O}_{8}(171 \mathrm{mg}, 0.75 \mathrm{mmol}, 3$ equiv) in $1 \mathrm{~mL}$ of dry DMSO. The reaction was irradiated with two $30 \mathrm{~W}$ Kessil blue LED lamps for $6 \mathrm{~h}$ at $30^{\circ} \mathrm{C}$. DCM was used in place of $\mathrm{Et}_{2} \mathrm{O}$ 
during the work-up. Purified by flash column chromatography (Hex $\rightarrow$ 1:1 Hex:EtOAc) to afford 44 as a colorless solid in $51 \%$ yield $(45 \mathrm{mg})$.

${ }^{\mathbf{1}} \mathbf{H}$ NMR: $\left(600 \mathrm{MHz}, \mathrm{CDCl}_{3}\right) \delta 8.07(\mathrm{~s}, 2 \mathrm{H}), 7.04(\mathrm{~s}, 2 \mathrm{H}), 4.07(\mathrm{~s}, 6 \mathrm{H}), 1.58(\mathrm{~s}, 18 \mathrm{H}) .{ }^{13} \mathbf{C ~ N M R :}$ $\left(151 \mathrm{MHz}, \mathrm{CDCl}_{3}\right) \delta 170.4,162.5,145.6,119.8,118.2,98.9,55.6,39.2,30.5$. Rf: 0.53 (1:1 Hex:EtOAc) HRMS (m/z): Calculated for $\mathrm{C}_{22} \mathrm{H}_{28} \mathrm{~N}_{2} \mathrm{O}_{2}\left([\mathrm{M}+\mathrm{H}]^{+}\right)$: 353.2229, found: 353.2225. IR (neat, $\mathbf{c m}^{-1}$ ): 2961, 2362, 1589, 1544, 1457, 1407, 1354, 1240, 1052, 924, 841, 824.

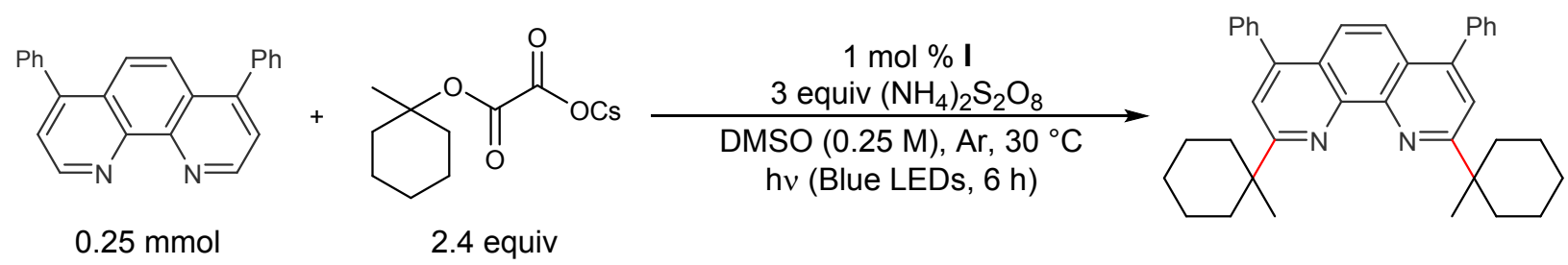

2,9-Bis(1-methylcyclohexyl)-4,7-diphenyl-1,10-phenanthroline (45): Prepared according to GP1 from bathophenanthroline (83 mg, $0.25 \mathrm{mmol}, 1$ equiv), cesium 2-((1methylcyclohexyl)oxy)-2-oxoacetate ${ }^{2}\left(191 \mathrm{mg}, 0.6 \mathrm{mmol}, 2.4\right.$ equiv), $\operatorname{Ir}\left[\mathrm{dF}\left(\mathrm{CF}_{3}\right) \text { ppy }\right]_{2}(\mathrm{dtbbpy}) \mathrm{PF}_{6}$ (2.8 mg, $0.0025 \mathrm{mmol}, 1 \mathrm{~mol} \%$ ) and $\left(\mathrm{NH}_{4}\right)_{2} \mathrm{~S}_{2} \mathrm{O}_{8}(171 \mathrm{mg}, 0.75 \mathrm{mmol}, 3$ equiv) in $1 \mathrm{~mL}$ of dry DMSO. The reaction was irradiated with two $30 \mathrm{~W}$ Kessil blue LED lamps for $6 \mathrm{~h}$ at $30{ }^{\circ} \mathrm{C}$. DCM was used in place of $\mathrm{Et}_{2} \mathrm{O}$ during the work-up. Purified by flash column chromatography (Hex $\rightarrow$ 9:1 Hex:EtOAc) to afford $\mathbf{4 5}$ as a colorless solid in 63\% yield (83 $\mathrm{mg})$.

${ }^{1}$ H NMR: $\left(600 \mathrm{MHz}, \mathrm{CDCl}_{3}\right) \delta 7.74(\mathrm{~s}, 2 \mathrm{H}), 7.60(\mathrm{~s}, 2 \mathrm{H}), 7.55-7.50(\mathrm{~m}, 8 \mathrm{H}), 7.49-7.45(\mathrm{~m}, 2 \mathrm{H})$, 2.64-2.55 (m, 4H), 1.77-1.62 (m, 12H), 1.57-1.42 (m, 10H). ${ }^{13} \mathrm{C}$ NMR: $\left(151 \mathrm{MHz}, \mathrm{CDCl}_{3}\right) \delta$ $167.9,148.2,146.0,139.3,129.9,128.6,128.2,124.6,123.0,120.5,42.2,37.9,30.0,26.7,23.3$. Rf: 0.55 (8:1 Hex:EtOAc) HRMS (m/z): Calculated for $\mathrm{C}_{38} \mathrm{H}_{40} \mathrm{~N}_{2}\left([\mathrm{M}+\mathrm{H}]^{+}\right)$: 525.3270, found: 525.3255. IR (neat, $\left.\mathbf{c m}^{-1}\right):$ 2922, 2852, 2360, 1565, 1540, 1478, 1444, 890, 848, 830, 768. 


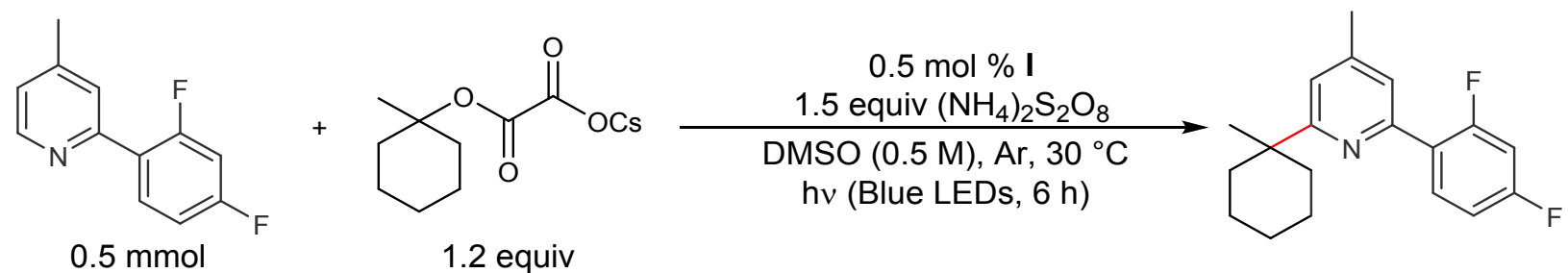

2-(2,4-Difluorophenyl)-4-methyl-6-(1-methylcyclohexyl)pyridine (46): Prepared according to

GP1 from 2-(difluorophenyl)-4-methylpyridine ${ }^{18}$ (103 mg, $0.5 \mathrm{mmol}, 1$ equiv), cesium 2-((1methylcyclohexyl)oxy)-2-oxoacetate ${ }^{2}\left(191 \mathrm{mg}, 0.6 \mathrm{mmol}, 1.2\right.$ equiv), $\operatorname{Ir}\left[\mathrm{dF}\left(\mathrm{CF}_{3}\right) \text { ppy }\right]_{2}\left(\mathrm{dtbbpy} \mathrm{PF}_{6}\right.$ (2.8 mg, $0.0025 \mathrm{mmol}, 0.5 \mathrm{~mol} \%$ ) and $\left(\mathrm{NH}_{4}\right)_{2} \mathrm{~S}_{2} \mathrm{O}_{8}(171 \mathrm{mg}, 0.75 \mathrm{mmol}, 1.5$ equiv) in $1 \mathrm{~mL}$ of dry DMSO. The react ion was irradiated with two $30 \mathrm{~W}$ Kessil blue LED lamps for $6 \mathrm{~h}$ at $30{ }^{\circ} \mathrm{C}$. EtOAc was used in place of $\mathrm{Et}_{2} \mathrm{O}$ during the work-up. Purified by flash column chromatography $(\mathrm{Hex} \rightarrow$ 9:1 EtOAc:Hex) to afford $\mathbf{4 6}$ as a clear oil in 27\% yield (41 mg).

${ }^{1}$ H NMR: $\left(600 \mathrm{MHz}, \mathrm{CDCl}_{3}\right) \delta 8.16-8.11(\mathrm{~m}, 1 \mathrm{H}), 7.41(\mathrm{~s}, 1 \mathrm{H}), 7.09(\mathrm{~s}, 1 \mathrm{H}), 6.98(\mathrm{dt}, J=8.3,2.4$ $\mathrm{Hz}, 1 \mathrm{H}), 6.90-6.85(\mathrm{~m}, 1 \mathrm{H}), 2.39(\mathrm{~s}, 3 \mathrm{H}), 2.29-2.21(\mathrm{~s}, 1 \mathrm{H}), 1.61-1.54(\mathrm{~m}, 4 \mathrm{H}), 1.47-1.41(\mathrm{~m}, 4 \mathrm{H})$, $1.24(\mathrm{~s}, 3 \mathrm{H}) .{ }^{13} \mathrm{C}$ NMR: $\left(151 \mathrm{MHz}, \mathrm{CDCl}_{3}\right) \delta 168.2,163.0(\mathrm{dd}, J=250,12 \mathrm{~Hz}), 160.9(\mathrm{dd}, J=252$ $12 \mathrm{~Hz}), 150.8(\mathrm{~d}, J=2.6 \mathrm{~Hz}), 147.5,132.6(\mathrm{dd}, J=9.5,4.8 \mathrm{~Hz}), 124.6(\mathrm{~d}, J=11 \mathrm{~Hz}), 121.9(\mathrm{~d}, J=10$ Hz), 119.8, 111.8 (dd, $J=21,3.5$ Hz), 104.3 (dd, $J=27,25 \mathrm{~Hz}), 41.0,37.3$, 29.5, 26.5, 23.0, 21.6. Rf: 0.77 (8:1 Hex:EtOAc) HRMS (m/z): Calculated for $\mathrm{C}_{19} \mathrm{H}_{21} \mathrm{~F}_{2} \mathrm{~N}\left([\mathrm{M}+\mathrm{H}]^{+}\right)$: 302.1720, found: 302.1719. IR (neat, $\left.\mathbf{c m}^{-1}\right):$ 2926, 2857, 2359, 2340, 1603, 1563, 1504, 1446, 1264, 1138, 970, 847. 


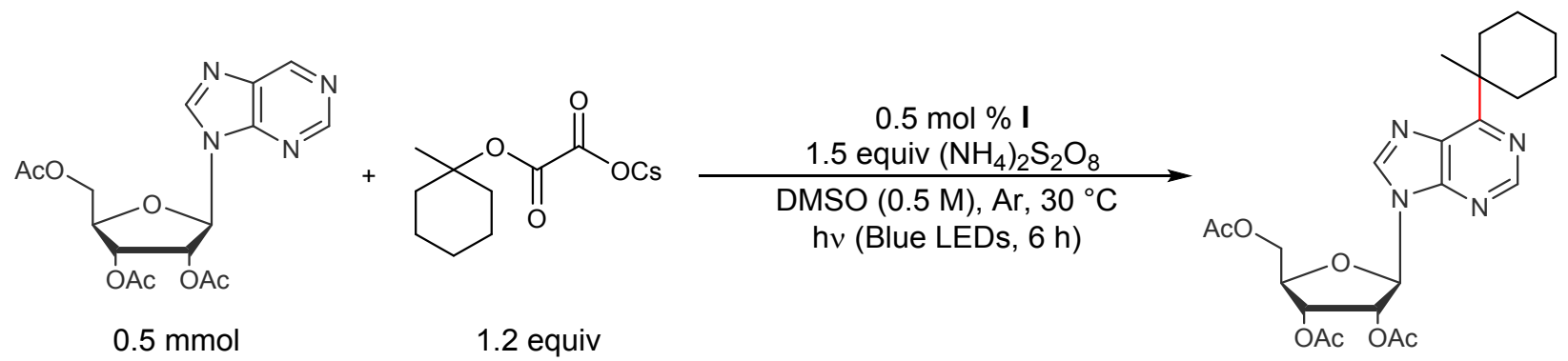

$(2 R, \quad 3 R, \quad 4 R, \quad 5 R)-2-($ acetoxymethyl)-5-(6-(1-methylcyclohexyl)-9H-purin-9-yl)tetra-

hydrofuran-3,4-diyl diacetate (47): Prepared according to GP1 from $(2 R, 3 R, 4 R, 5 R)-2$ (acetoxymethyl)-5-(9H-purin-9-yl)tetrahydrofuran-3,4-diyl diacetate ${ }^{19}$ (1 mL of a $0.5 \mathrm{M}$ solution in DMSO, 1 equiv), cesium 2-((1-methylcyclohexyl)oxy)-2-oxoacetate ${ }^{2}$ (191 mg, 0.6 mmol, 1.2 equiv), $\operatorname{Ir}\left[\mathrm{dF}\left(\mathrm{CF}_{3}\right) \mathrm{ppy}\right]_{2}(\mathrm{dtbbpy}) \mathrm{PF}_{6}(2.8 \mathrm{mg}, 0.0025 \mathrm{mmol}, 0.5 \mathrm{~mol} \%)$ and $\left(\mathrm{NH}_{4}\right)_{2} \mathrm{~S}_{2} \mathrm{O}_{8}(171 \mathrm{mg}$, $0.75 \mathrm{mmol}, 1.5$ equiv). The reaction was irradiated with two $30 \mathrm{~W}$ Kessil blue LED lamps for $6 \mathrm{~h}$ at $30{ }^{\circ} \mathrm{C}$. EtOAc was used in place of $\mathrm{Et}_{2} \mathrm{O}$ during the work-up. Purified by flash column chromatography (Hex $\rightarrow$ 1:1 EtOAc:Hex) to afford 47 as a clear oil in $66 \%$ yield (156 mg).

${ }^{1}$ H NMR: $\left(600 \mathrm{MHz}, \mathrm{CDCl}_{3}\right) \delta 8.91(\mathrm{~s}, 1 \mathrm{H}), 8.13(\mathrm{~s}, 1 \mathrm{H}), 6.25(\mathrm{~d}, J=5.2 \mathrm{~Hz}, 1 \mathrm{H}), 5.97(\mathrm{t}, J=5.4$ Hz, 1H), 5.70 (t, J=5.1 Hz, 1H), 4.47-4.43 (m, 2H), 4.39-4.35 (m, 1H), 2.73-2.66 (m, 2H), 2.14 (s, 3H), 2.12 (s, 3H), $2.09(\mathrm{~s}, 3 \mathrm{H}), 1.72-1.60(\mathrm{~m}, 4 \mathrm{H}), 1.49-1.36(\mathrm{~m}, 7 \mathrm{H}) .{ }^{13} \mathrm{C}$ NMR: (151 MHz, $\left.\mathrm{CDCl}_{3}\right) \delta 170.5,169.74,169.71,169.5,152.1,151.2,140.6,132.7,86.5,80.4,73.2,70.7,63.2$ 42.6, 36.6, 28.0, 26.2 23.1, 20.9, 20.7, 20.6. Rf: 0.14 (1:1 Hex:EtOAc) HRMS (m/z): Calculated for $\mathrm{C}_{23} \mathrm{H}_{30} \mathrm{~N}_{4} \mathrm{O}_{7}\left([\mathrm{M}+\mathrm{H}]^{+}\right)$: 475.2193, found: 475.2183. IR (neat, $\left.\mathbf{c m}^{-1}\right)$ : 2929, 2856, 1747, 1566, 1372, 1328, 1212, 1097, 1046, 913, 733. 


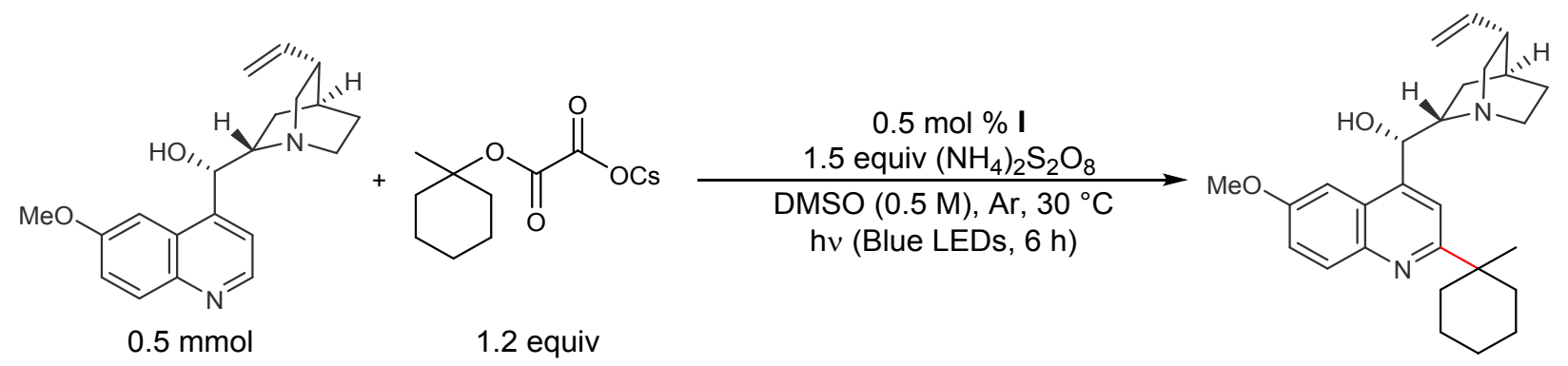

(S)-(6-methoxy-2-(1-methylcyclohexyl)quinolin-4-yl)((1S, 2S, 4S, 5R)-5-vinylquinuclidin-2-

yl)methanol (48): Prepared according to GP1 from Quinine (162 mg, $0.5 \mathrm{mmol}, 1$ equiv), cesium 2-((1-methylcyclohexyl)oxy)-2-oxoacetate ${ }^{2} \quad(191 \quad \mathrm{mg}, \quad 0.6 \quad \mathrm{mmol}, \quad 1.2 \quad$ equiv $)$, $\operatorname{Ir}\left[\mathrm{dF}\left(\mathrm{CF}_{3}\right) \text { ppy }\right]_{2}(\mathrm{dtbbpy}) \mathrm{PF}_{6}(2.8 \mathrm{mg}, 0.0025 \mathrm{mmol}, 0.5 \mathrm{~mol} \%)$ and $\left(\mathrm{NH}_{4}\right)_{2} \mathrm{~S}_{2} \mathrm{O}_{8}(171 \mathrm{mg}, 0.75$ mmol, 1.5 equiv) in $1 \mathrm{~mL}$ of dry DMSO. The reaction was irradiated with two $30 \mathrm{~W}$ Kessil blue LED lamps for $6 \mathrm{~h}$ at $30{ }^{\circ} \mathrm{C}$. EtOAc was used in place of $\mathrm{Et}_{2} \mathrm{O}$ during the work-up. Purified by flash column chromatography (Hex $\rightarrow$ 1:1 EtOAc:Hex) to afford 48 as a clear oil in 32\% yield (67 mg).

${ }^{1}$ H NMR: $\left(600 \mathrm{MHz}, \mathrm{CDCl}_{3}\right) \delta 7.77(\mathrm{~s}, 1 \mathrm{H}), 7.69(\mathrm{~d}, J=8.8 \mathrm{~Hz}, 1 \mathrm{H}), 6.91-6.85(\mathrm{~m}, 2 \mathrm{H}), 6.41(\mathrm{~s}$, 1H), 5.61-5.60 (m, 2H), 5.05-4.98 (m, 2H), 4.56-4.49 (m, 1H), $3.48(\mathrm{~s}, 3 \mathrm{H}), 3.40$ (app t, $J=12 \mathrm{~Hz}$ $1 \mathrm{H}), 3.29(\mathrm{t}, J=8.6 \mathrm{~Hz}, 1 \mathrm{H}), 3.13-3.06(\mathrm{~m}, 1 \mathrm{H}), 3.02-2.96(\mathrm{~m}, 1 \mathrm{H}), 2.71-2.64(\mathrm{~m}, 1 \mathrm{H}), 2.37-2.22$ (m, 3H), 2.07 (b.s., 1H), 1.88-1.80 (m, 1H), 1.74-1.56 (m, 4H), 1.52-1.39 (m, 4H), 1.33 (s, 3H). ${ }^{13}$ C NMR: (151 MHz, $\mathrm{CDCl}_{3}$, some peaks were doubled due to rotamers) $\delta 165.3,157.7,143.6$, $143.4,137.4,131.5,123.4,121.7,117.5,116.3,99.3,66.5,60.3,57.0,54.9,44.1,41.2,37.5,37.4$, 37.2 28.7, 27.3 26.5, 24.5, 23.0, 22.9, 18.1. Rf: 0.31 (9:1 DCM:MeOH) HRMS (m/z): Calculated for $\mathrm{C}_{27} \mathrm{H}_{36} \mathrm{~N}_{2} \mathrm{O}_{2}\left([\mathrm{M}+\mathrm{H}]^{+}\right)$: 421.2855, found: 421.2874. IR (neat, $\left.\mathbf{c m}^{-1}\right)$ : 3261, 2925, 2360, 2341, $1559,1506,1457,1233,1033,668$. 


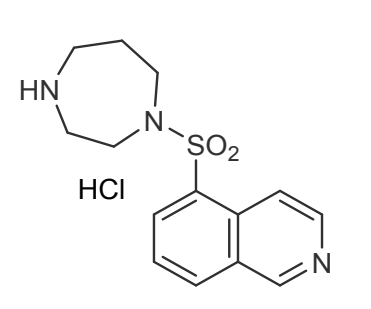

$0.5 \mathrm{mmol}$

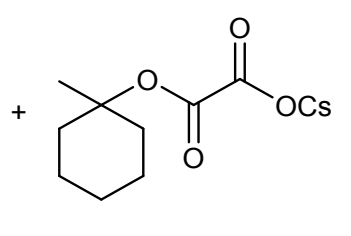

1.2 equiv

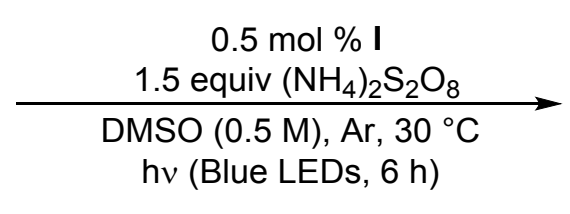

(n)

5-((1,4-Diazepan-1-yl)sulfonyl)-1-(1-methylcyclohexyl)isoquinoline (49): Prepared according

to GP1 from Fasudil·HCl (164 mg, 0.5 mmol, 1 equiv), cesium 2-((1-methylcyclohexyl)oxy)-2oxoacetate $^{2}$ (191 mg, $0.6 \mathrm{mmol}, 1.2$ equiv), $\operatorname{Ir}\left[\mathrm{dF}\left(\mathrm{CF}_{3}\right) \mathrm{ppy}\right]_{2}(\mathrm{dtbbpy}) \mathrm{PF}_{6}(2.8 \mathrm{mg}, 0.0025 \mathrm{mmol}$, $0.5 \mathrm{~mol} \%)$ and $\left(\mathrm{NH}_{4}\right)_{2} \mathrm{~S}_{2} \mathrm{O}_{8}(171 \mathrm{mg}, 0.75 \mathrm{mmol}, 1.5$ equiv) in $1 \mathrm{~mL}$ of dry DMSO. The reaction was irradiated with two $30 \mathrm{~W}$ Kessil blue LED lamps for $6 \mathrm{~h}$ at $30^{\circ} \mathrm{C}$. EtOAc was used in place of $\mathrm{Et}_{2} \mathrm{O}$ during the work-up. Purified by flash column chromatography (DCM $\rightarrow$ 95:5 DCM:MeOH) to afford 49 as a clear oil in $25 \%$ yield (49 $\mathrm{mg})$.

${ }^{1}$ H NMR: $\left(600 \mathrm{MHz}, \mathrm{CDCl}_{3}\right) \delta 8.85(\mathrm{~d}, J=8.8 \mathrm{~Hz}, 1 \mathrm{H}), 8.62(\mathrm{~d}, J=6.0 \mathrm{~Hz}, 1 \mathrm{H}), 8.25-8.21(\mathrm{~m}, 2 \mathrm{H})$, 7.60-7.56 (m, 1H), 3.73-3.69 (m, 2H), 3.59 (t, $J=6.5 \mathrm{~Hz}, 2 \mathrm{H}), 3.38-3.35(\mathrm{~m}, 2 \mathrm{H}), 3.34-3.30$ (m, 2H), $1.93-1.87(\mathrm{~m}, 2 \mathrm{H}), 1.67-1.60(\mathrm{~m}, 5 \mathrm{H}), 1.59-1.51(\mathrm{~m}, 3 \mathrm{H}), 1.46-1.38(\mathrm{~m}, 1 \mathrm{H}) .{ }^{13} \mathbf{C}$ NMR: (151 $\left.\mathrm{MHz}, \mathrm{CDCl}_{3}\right) \delta 168.5,142.6,135.6,133.6,132.6,131.6,127.2,123.8,115.7,50.1,50.0,47.3$, 47.2, 43.7, 39.4, 30.1, 27.4, 26.5, 23.0. Rf: 0.24 (9:1 DCM:MeOH) HRMS (m/z): Calculated for $\mathrm{C}_{21} \mathrm{H}_{29} \mathrm{~N}_{3} \mathrm{O}_{2} \mathrm{~S}\left([\mathrm{M}+\mathrm{H}]^{+}\right)$: 388.2059, found: 388.2068. IR (neat, $\mathbf{c m}^{-1}$ ): 2921, 2860, 2360, 2341, $1558,1540,1456,1327,1144,1033,913,743$. 


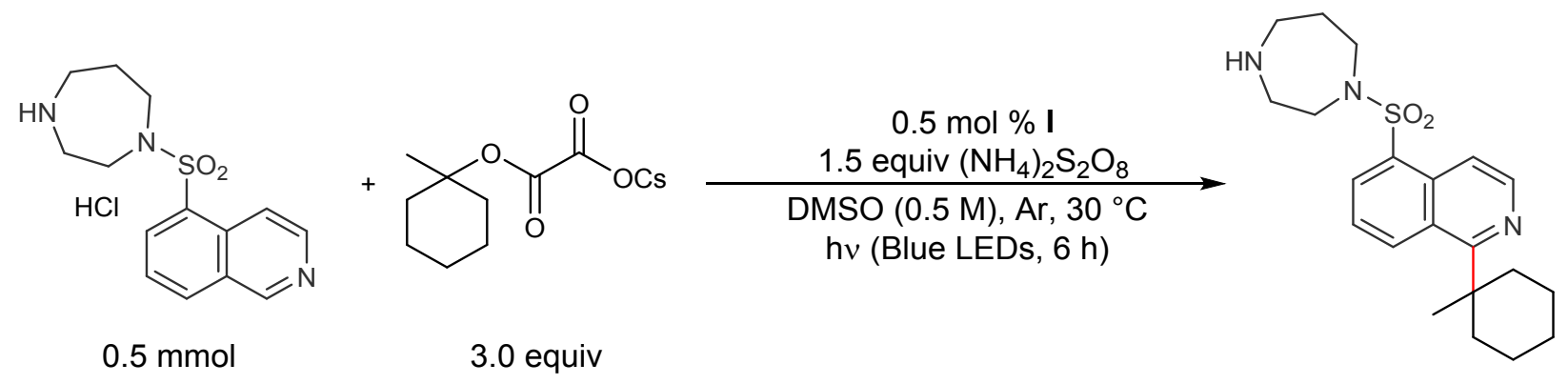

5-((1,4-Diazepan-1-yl)sulfonyl)-1-(1-methylcyclohexyl)isoquinoline (49): Prepared according

to GP1 from Fasudil $\cdot \mathrm{HCl}$ (66 mg, $0.2 \mathrm{mmol}, 1$ equiv), cesium 2-((1-methylcyclohexyl)oxy)-2oxoacetate $^{2}$ (191 mg, $0.6 \mathrm{mmol}, 3.0$ equiv), $\operatorname{Ir}\left[\mathrm{dF}\left(\mathrm{CF}_{3}\right) \text { ppy }\right]_{2}(\mathrm{dtbbpy}) \mathrm{PF}_{6}\left(2.8 \mathrm{mg}, 0.0025 \mathrm{mmol}_{\text {, }}\right.$ $1.25 \mathrm{~mol} \%)$ and $\left(\mathrm{NH}_{4}\right)_{2} \mathrm{~S}_{2} \mathrm{O}_{8}(171 \mathrm{mg}, 0.75 \mathrm{mmol}, 3.75$ equiv) in $1 \mathrm{~mL}$ of dry DMSO. The reaction was irradiated with two $30 \mathrm{~W}$ Kessil blue LED lamps for $6 \mathrm{~h}$ at $30^{\circ} \mathrm{C}$. EtOAc was used in place of $\mathrm{Et}_{2} \mathrm{O}$ during the work-up. Purified by flash column chromatography (DCM $\rightarrow$ 95:5 DCM:MeOH) to afford 49 as an off-white solid in 39\% yield (30 mg). Spectral data matched that previously reported. 


\section{Experimental Procedures for the Thermal Minisci Reaction}<smiles>Cc1c[cH+]cnc2ccccc12</smiles>

$$
0.5 \mathrm{mmol} \quad 1.2 \text { equiv }
$$

2-(tert-Butyl)-4-methylquinoline (3): Prepared according to GP2 from lepidine $(0.5 \mathrm{mmol}, 66 \mu \mathrm{L}, 1$ equiv), cesium 2-(tert-butoxy)-2-oxoacetate ${ }^{2}$ (167 mg, $0.6 \mathrm{mmol}, 1.2$ equiv) and $\left(\mathrm{NH}_{4}\right)_{2} \mathrm{~S}_{2} \mathrm{O}_{8}(171$ mg, $0.75 \mathrm{mmol}, 1.5$ equiv) in $1 \mathrm{~mL}$ of dry DMSO. The reaction was stirred for $10 \mathrm{~min}$ at $65{ }^{\circ} \mathrm{C}$. Purified by flash column chromatography (Hex $\rightarrow$ 9:1 Hex:EtOAc) to afford $\mathbf{3}$ as a clear oil in $72 \%$ yield (72 mg). Spectral data matched that previously reported.<smiles>CC1=C[C+]=CN=C1c1ccccc1</smiles>

$0.5 \mathrm{mmol}$<smiles>CC1(OC(=O)C(=O)O)CCCCC1</smiles>

1.2 equiv<smiles>Cc1cc(C2(C)CCCCC2)nc2ccccc12</smiles>

4-Methyl-2-(1-methylcyclohexyl)quinoline (4): Prepared according to GP2 from lepidine (0.5 mmol,

$66 \mu \mathrm{L}, 1$ equiv), lithium 2-((1-methylcyclohexyl)oxy)-2-oxoacetate ${ }^{2}$ (115 mg, $0.6 \mathrm{mmol}, 1.2$ equiv) and $\left(\mathrm{NH}_{4}\right)_{2} \mathrm{~S}_{2} \mathrm{O}_{8}(171 \mathrm{mg}, 0.75 \mathrm{mmol}, 1.5$ equiv) in $1 \mathrm{~mL}$ of dry DMSO. The reaction was stirred for $10 \mathrm{~min}$ at $65^{\circ} \mathrm{C}$. Purified by flash column chromatography (Hex $\rightarrow$ 9:1 Hex:EtOAc) to afford 4 as a clear oil in 50\% yield $(60 \mathrm{mg})$. Spectral data matched that previously reported. 
<smiles>CC1=C[C+]=CN=C1c1ccccc1</smiles>

$0.5 \mathrm{mmol}$<smiles>CC1(OC(=O)C(=O)OCc2ccccc2)CCCC1</smiles>

1.2 equiv

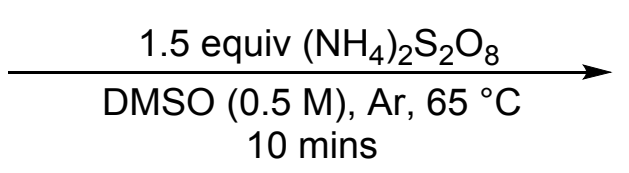

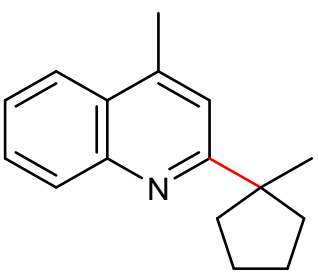

4-Methyl-2-(1-methylcyclopentyl)quinoline (6): Prepared according to GP2 from lepidine (0.5 mmol, $66 \mu \mathrm{L}, 1$ equiv), cesium 2-((1-methylcyclopentyl)oxy)-2-oxoacetate ${ }^{2}$ (182 mg, 0.6 mmol, 1.2 equiv) and $\left(\mathrm{NH}_{4}\right)_{2} \mathrm{~S}_{2} \mathrm{O}_{8}(171 \mathrm{mg}, 0.75 \mathrm{mmol}, 1.5$ equiv) in $1 \mathrm{~mL}$ of dry DMSO. The reaction was stirred for $10 \mathrm{~min}$ at $65^{\circ} \mathrm{C}$. Purified by flash column chromatography (Hex $\rightarrow$ 9:1 Hex:EtOAc) to afford 6 as a clear oil in 53\% yield $(60 \mathrm{mg})$. Spectral data matched that previously reported.<smiles>Cc1cc[nH+]c2ccccc12</smiles>

\section{$0.5 \mathrm{mmol}$ \\ 1.2 equiv}

2-((3r, 5r, 7r)-Adamantan-1-yl)-4-methylquinoline (7): Prepared according to GP2 from lepidine (0.5 mmol, $66 \mu \mathrm{L}, 1$ equiv), lithium 2-(((3s,5s,7s)-adamantan-1-yl)oxy)-2-oxoacetate (138 mg, 0.6 mmol, 1.2 equiv) and $\left(\mathrm{NH}_{4}\right)_{2} \mathrm{~S}_{2} \mathrm{O}_{8}(171 \mathrm{mg}, 0.75 \mathrm{mmol}, 1.5$ equiv $)$ in $1 \mathrm{~mL}$ of dry DMSO. The reaction was stirred for $10 \mathrm{~min}$ at $65^{\circ} \mathrm{C}$. Purified by flash column chromatography (Hex $\rightarrow$ 9:1 Hex:EtOAc) to afford 7 as a colorless solid in 69\% yield $(96 \mathrm{mg})$. Spectral data matched that previously reported. 
<smiles>Cc1ccnc2ccccc12</smiles>

$0.5 \mathrm{mmol}$<smiles>CC1(OC(=O)C(=O)O)CCN(C(=O)OCc2ccccc2)CC1</smiles>

1.2 equiv<smiles>Cc1cc(C2(C)CCN(C(=O)OCc3ccccc3)CC2)nc2ccccc12</smiles>

tert-Butyl 4-methyl-4-(4-methylquinolin-2-yl)piperidine-1-carboxylate (8): Prepared according to GP2 from lepidine (0.5 mmol, $66 \mu \mathrm{L}, 1$ equiv), lithium 2-((1- (tert-butoxycarbonyl)-4methylpiperidin-4-yl)oxy)-2-oxoacetate (176 mg, $0.6 \mathrm{mmol}, 1.2$ equiv) and $\left(\mathrm{NH}_{4}\right)_{2} \mathrm{~S}_{2} \mathrm{O}_{8}(171 \mathrm{mg}$, $0.75 \mathrm{mmol}, 1.5$ equiv) in $1 \mathrm{~mL}$ of dry DMSO. The reaction was stirred for $10 \mathrm{~min}$ at $65^{\circ} \mathrm{C}$. Purified by flash column chromatography (Hex $\rightarrow$ 9:1 Hex:EtOAc) to afford $\mathbf{8}$ as a clear oil in 65\% yield (110 mg). Spectral data matched that previously reported.<smiles>Cc1ccnc2ccccc12</smiles>

$0.5 \mathrm{mmol}$<smiles>COC(=O)C(=O)OC1(C)CCC(F)(F)CC1</smiles>

1.2 equiv

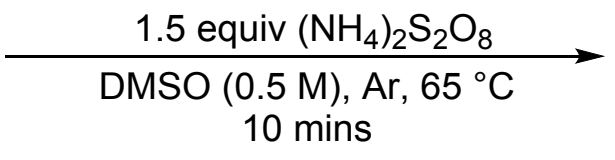

10 mins<smiles>Cc1cc(C2(C)CCC(F)(F)CC2)nc2ccccc12</smiles>

2-(4,4-Difluoro-1-methylcyclohexyl)-4-methylquinoline (10): Prepared according to GP2 from lepidine (0.5 mmol, $66 \mu \mathrm{L}, 1$ equiv), cesium 2-((4,4-difluoro-1-methylcyclohexyl)oxy)-2oxoacetate (212 mg, $0.6 \mathrm{mmol}, 1.2$ equiv) and $\left(\mathrm{NH}_{4}\right)_{2} \mathrm{~S}_{2} \mathrm{O}_{8}(171 \mathrm{mg}, 0.75 \mathrm{mmol}, 1.5$ equiv) in 1 $\mathrm{mL}$ of dry DMSO. The reaction was stirred for 10 min at $65^{\circ} \mathrm{C}$. Purified by flash column chromatography (Hex $\rightarrow$ 9:1 Hex:EtOAc) to afford the desired compound as a clear oil in $47 \%$ yield (65 mg). Spectral data matched that previously reported. 


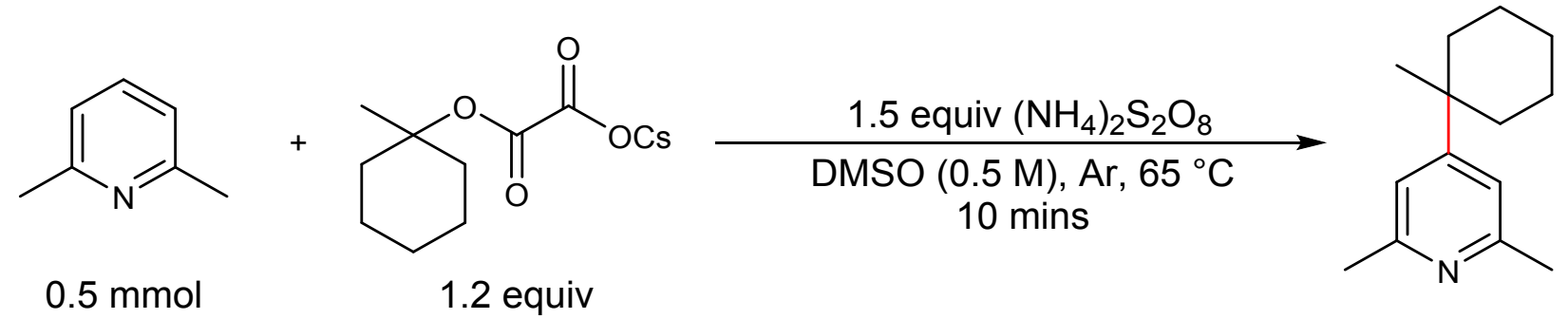

2,6-Dimethyl-4-(1-methylcyclohexyl)pyridine (27): Prepared according to GP2 from 2,6-lutidine (58 $\mu \mathrm{L}, 0.5 \mathrm{mmol}, 1$ equiv), cesium 2-((1-methylcyclohexyl)oxy)-2-oxoacetate ${ }^{2}(191 \mathrm{mg}, 0.6 \mathrm{mmol}$, 1.2 equiv) and $\left(\mathrm{NH}_{4}\right)_{2} \mathrm{~S}_{2} \mathrm{O}_{8}(171 \mathrm{mg}, 0.75 \mathrm{mmol}, 1.5$ equiv) in $1 \mathrm{~mL}$ of dry DMSO. The reaction was stirred for 10 mins at $65{ }^{\circ} \mathrm{C}$. Purified by flash column chromatography (Hex $\rightarrow$ 9:1 Hex:EtOAc) to afford 27 as a clear oil in $12 \%$ yield $(12 \mathrm{mg})$. Spectral data matched that previously reported.<smiles>COC(=O)C1=CN=C[C+]=C1</smiles>

$0.5 \mathrm{mmol}$<smiles>CCOC(=O)C(=O)OC1(C)CCCCC1</smiles>

1.2 equiv

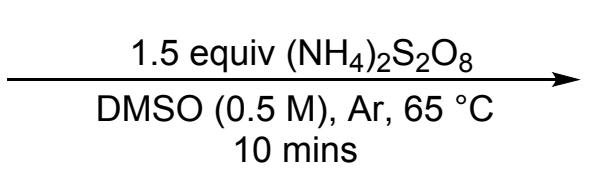

10 mins<smiles>COC(=O)c1ccc(C2(C)CCCCC2)nc1</smiles>

Methyl 6-(1-methylcyclohexyl)nicotinate (32): Prepared according to GP2 from methyl nicotinate (69 mg, 0.5 mmol, 1 equiv), cesium 2-((1-methylcyclohexyl)oxy)-2-oxoacetate ${ }^{2}$ (191 mg, 0.6 mmol, 1.2 equiv) and $\left(\mathrm{NH}_{4}\right)_{2} \mathrm{~S}_{2} \mathrm{O}_{8}(171 \mathrm{mg}, 0.75 \mathrm{mmol}, 1.5$ equiv) in $1 \mathrm{~mL}$ of dry DMSO. The reaction was stirred for 10 mins at $65^{\circ} \mathrm{C}$. Purified by flash column chromatography (Hex $\rightarrow$ 9:1 Hex:EtOAc) to afford 32 as a colorless solid in $45 \%$ yield $(53 \mathrm{mg}) .{ }^{20}$ Spectral data matched that previously reported. 


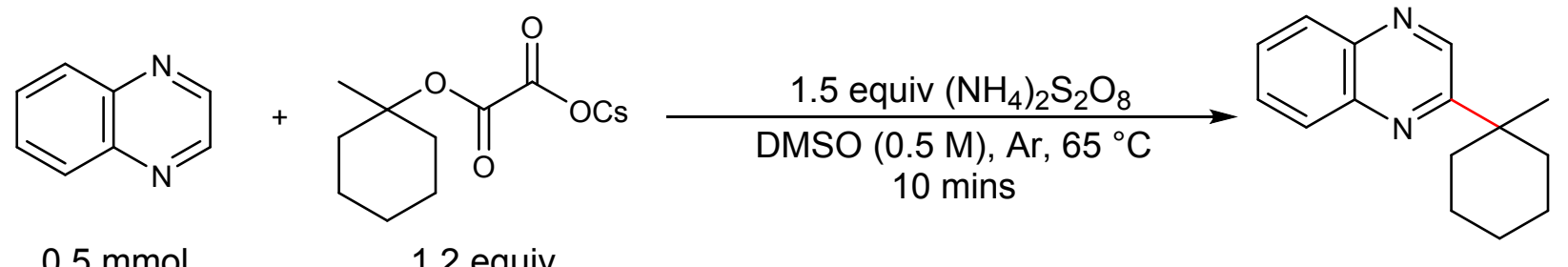

2-(1-Methylcyclohexyl)quinoxaline (35): Prepared according to GP2 from quinoxaline (65 mg, 0.5

mmol, 1 equiv), cesium 2-((1-methylcyclohexyl)oxy)-2-oxoacetate ${ }^{2}$ (191 mg, 0.6 mmol, 1.2 equiv) and $\left(\mathrm{NH}_{4}\right)_{2} \mathrm{~S}_{2} \mathrm{O}_{8}(171 \mathrm{mg}, 0.75 \mathrm{mmol}, 1.5$ equiv) in $1 \mathrm{~mL}$ of dry DMSO. The reaction was stirred for 10 mins at $65^{\circ} \mathrm{C}$. Purified by flash column chromatography (Hex $\rightarrow$ 9:1 Hex:EtOAc) to afford 35 as a clear oil in 61\% yield $(69 \mathrm{mg})$. Spectral data matched that previously reported.

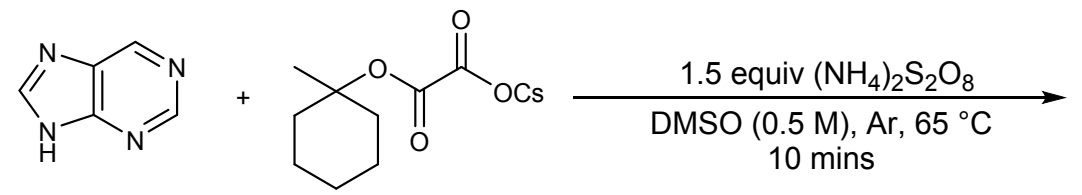

$0.5 \mathrm{mmol}$

1.2 equiv<smiles>CC1(c2ncnc3[nH]cnc23)CCCCC1</smiles>

38<smiles>CC1(c2nc(C3(C)CCCCC3)c3nc[nH]c3n2)CCCCC1</smiles>

6-(1-Methylcyclohexyl)-9H-purine (38): Prepared according to GP2 from purine (60 mg, 0.5 mmol, 1 equiv), cesium 2-((1-methylcyclohexyl)oxy)-2-oxoacetate ${ }^{2}$ (191 mg, 0.6 mmol, 1.2 equiv) and $\left(\mathrm{NH}_{4}\right)_{2} \mathrm{~S}_{2} \mathrm{O}_{8}(171 \mathrm{mg}, 0.75 \mathrm{mmol}, 1.5$ equiv) in $1 \mathrm{~mL}$ of dry DMSO. The reaction was stirred for 10 mins at $65^{\circ} \mathrm{C}$. EtOAc was used in place of $\mathrm{Et}_{2} \mathrm{O}$ during the work-up. Purified by flash column chromatography (DCM $\rightarrow$ 99:1 DCM:MeOH) to afford:

6-(1-methylcyclohexyl)-9H-purine (38) as a colorless solid in $82 \%$ yield (89 $\mathrm{mg})$. Spectral data matched that previously reported.

2,6-di-(1-methylcyclohexyl)-9H-purine was isolated also as a colorless solid in $11 \%$ yield from purine $(17 \mathrm{mg})$. Spectral data matched that previously reported. 


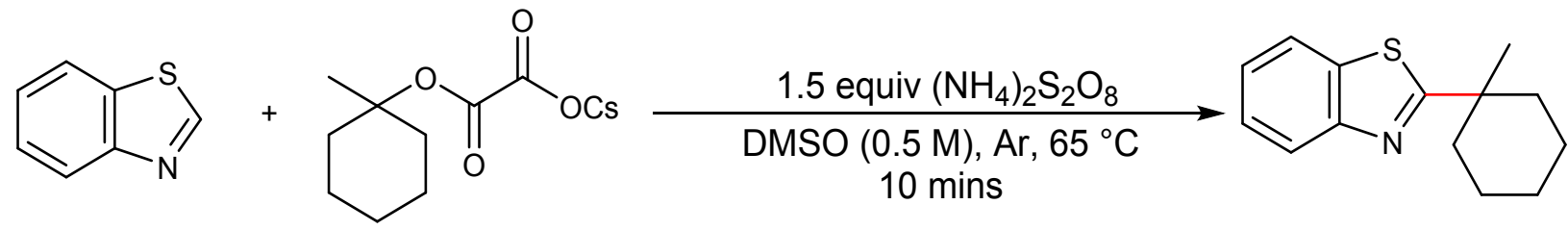

$0.5 \mathrm{mmol}$

1.2 equiv

2-(1-Methylcyclohexyl)benzo[d] thiazole (40): Prepared according to GP2 from benzothiazole (55 $\mu \mathrm{L}, 0.5 \mathrm{mmol}, 1$ equiv), cesium 2-((1-methylcyclohexyl)oxy)-2-oxoacetate ${ }^{2}$ (191 mg, 0.6 mmol, 1.2 equiv) and $\left(\mathrm{NH}_{4}\right)_{2} \mathrm{~S}_{2} \mathrm{O}_{8}(171 \mathrm{mg}, 0.75 \mathrm{mmol}, 1.5$ equiv) in $1 \mathrm{~mL}$ of dry DMSO. The reaction was stirred for 10 mins at $65^{\circ} \mathrm{C}$. Purified by flash column chromatography $(\mathrm{Hex} \rightarrow 9: 1$ Hex:EtOAc) to afford 40 as a clear oil in $47 \%$ yield $(54 \mathrm{mg})$. Spectral data matched that previously reported.

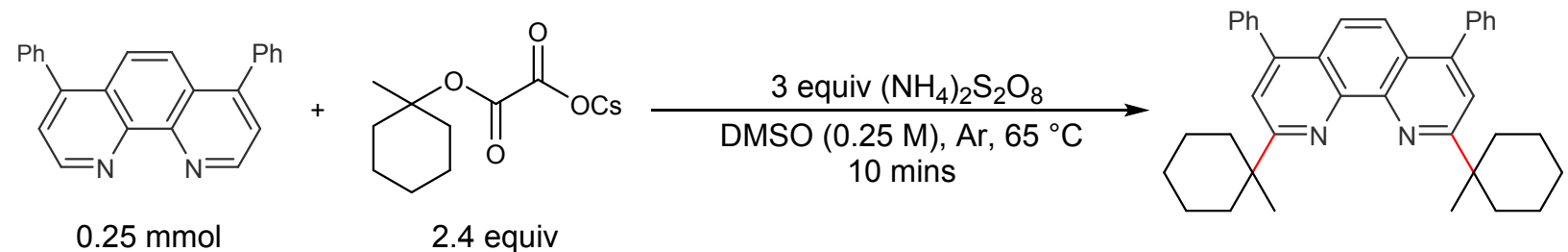

2,9-Di-(1-methylcyclohexyl)-4,7-diphenyl-1,10-phenanthroline (45): Prepared according to GP2 from bathophenanthroline (83 mg, $0.25 \mathrm{mmol}, 1$ equiv), cesium 2-((1-methylcyclohexyl)oxy)-2oxoacetate ${ }^{2}$ (191 mg, 0.6 mmol, 2.4 equiv) and $\left(\mathrm{NH}_{4}\right)_{2} \mathrm{~S}_{2} \mathrm{O}_{8}(171 \mathrm{mg}, 0.75 \mathrm{mmol}, 3$ equiv) in $1 \mathrm{~mL}$ of dry DMSO. The reaction was stirred for 10 mins at $65{ }^{\circ} \mathrm{C}$. DCM was used in place of $\mathrm{Et}_{2} \mathrm{O}$ during the work-up. Purified by flash column chromatography (Hex $\rightarrow$ 9:1 Hex:EtOAc) to afford 45 as a colorless solid in 55\% yield $(72 \mathrm{mg})$. Spectral data matched that previously reported. 


\section{N. Cyclic Voltammetry}

Conditions for cyclic voltammetry measurements: scan rate $=100 \mathrm{mV} \mathrm{s}^{-1} ; 10 \mathrm{mM}$ oxalate in $\mathrm{N}_{2}$ degassed $\mathrm{MeCN}$ containing $100 \mathrm{mM} \mathrm{Bu}_{4} \mathrm{NPF}_{6}$ as the supporting electrolyte; $1 \mathrm{~mm}$ diameter glassy carbon disk working electrode; glassy carbon rod counter electrode; $\mathrm{Ag} / \mathrm{Ag}^{+} \mathrm{pseudo-}^{-}$ reference electrode separated from the bulk solution by a vycor frit in a solution of $100 \mathrm{mM}$ $\mathrm{Bu}_{4} \mathrm{NPF}_{6} ; \mathrm{Fc} / \mathrm{Fc}^{+}$redox couple as internal reference $(0.41 \mathrm{~V}$ vs. SCE).

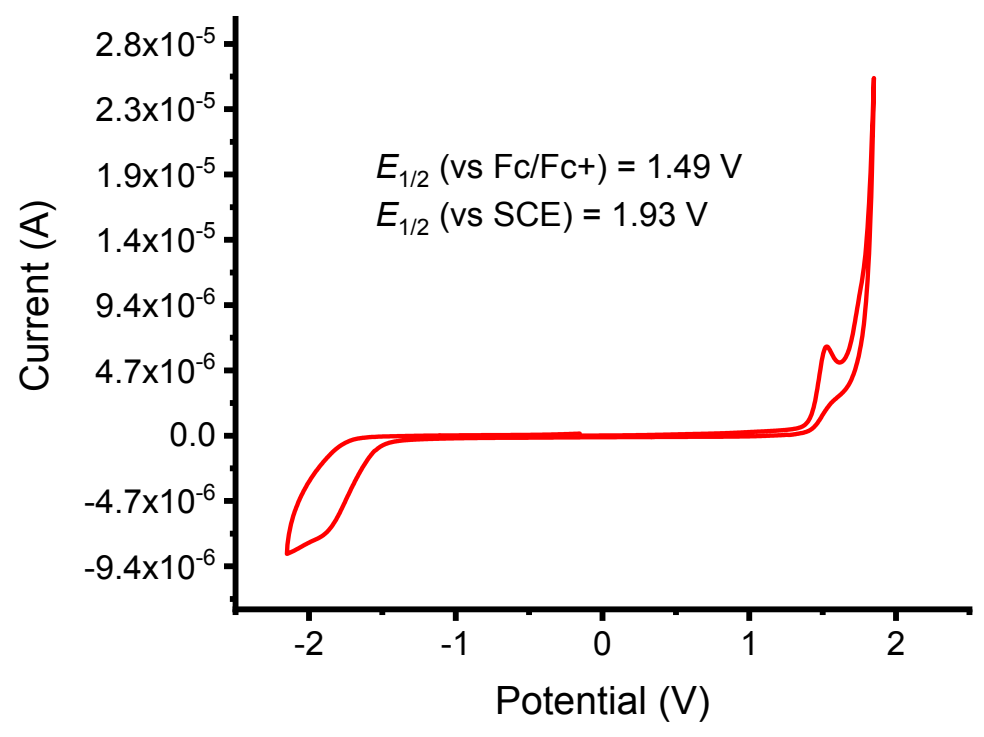

Figure S11. Cyclic voltammogram of 2-((2-methyl-4-phenylbutan-2-yl)oxy)-2-oxoacetic acid referenced to $\mathrm{Fc} / \mathrm{Fc}^{+}$. 


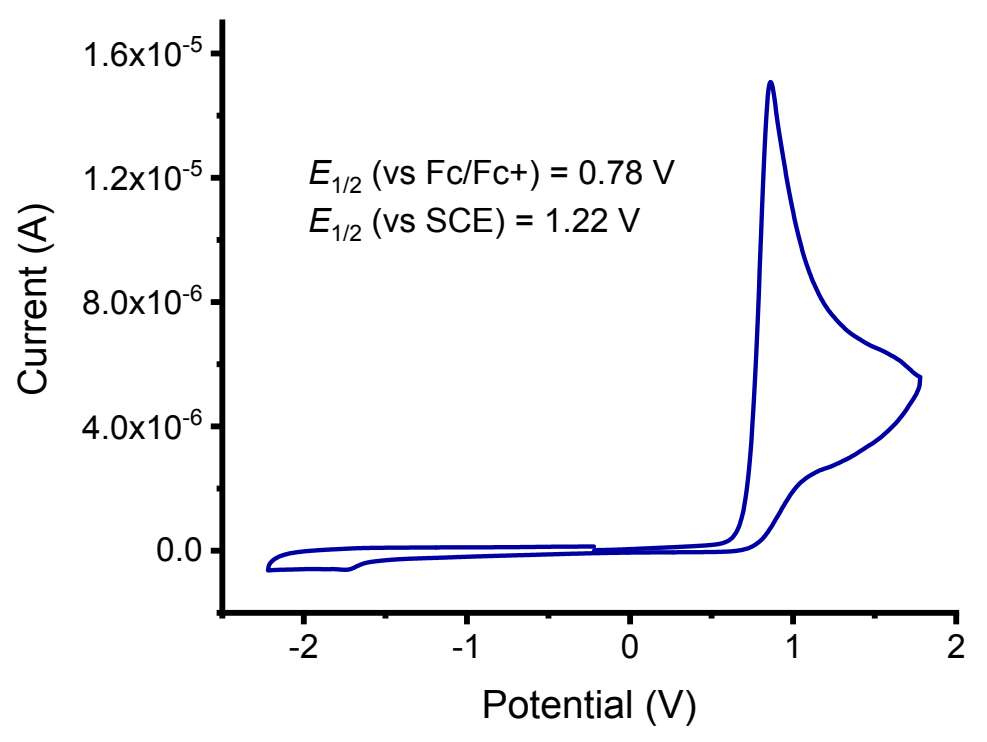

Figure S12. Cyclic voltammogram of cesium 2-((2-methyl-4-phenylbutan-2-yl)oxy)-2-oxoacetate referenced to $\mathrm{Fc} / \mathrm{Fc}^{+}$. 


\section{O. References}

1) Lowry, M. S.; Hudson, W. R.; Pascal, R. A. Jr.; Bernhard, S. Accelerated Luminophore Discovery through Combinatorial Synthesis. J. Am. Chem. Soc. 2004, 126, 14129-14135.

2) Nawrat, C. C.; Jamison, C. R.; Slutskyy, Y.; MacMillan, D. W. C.; Overman, L. E. Oxalates as Activating Groups for Alcohols in Visible Light Photoredox Catalysis: Formation of Quaternary Centers by Redox-Neutral Fragment Coupling. J. Am. Chem. Soc. 2015, 137, 11270-11273.

3) Furche F.; Ahlrichs, R.; Hättig, C.; Klopper, W.; Sierka, M.; Weigend, F. Turbomole. WIREs Comput. Mol. Sci. 2014, 4, 91-100.

4) Staroverov, V. N.; Scuseria, G. E.; Tao, J.; Perdew, J. P. Comparative Assessment of a New Nonempirical Density Functional: Molecules and Hydrogen-Bonded Complexes. J. Chem. Phys. 2003, 119, 12129-12137.

5) (a) Grimme, S.; Antony, J.; Ehrlich, S.; Krieg, H. A Consistent and Accurate Ab Initio Parametrization of Density Functional Dispersion Correction (DFT-D) for the 94 Elements HPu. J. Chem. Phys. 2010, 132, 154104; (b) Grimme, S.; Ehrlich S.; Goerigk L. Effect of the Damping Function in Dispersion Corrected Density Functional Theory J. Comput. Chem. 2011, 32, 1456-1465.

6) Weigend, F.; Ahlrichs, R. Phys. Chem. Chem. Phys. 2005, 7, 3297-3305.

7) Schäfer, A.; Klamt, A.; Sattel, D.; Lohrenz, J. C. W.; Eckert, F. Phys. Chem. Chem. Phys. 2000, 2, 2187-2193.

8) Sierka, M.; Hogekamp, A.; Ahlrichs, R. COSMO Implementation in TURBOMOLE: Extension of an Efficient Quantum Chemical Code Towards Liquid Systems J. Chem. Phys. 2003, 118, 9136-9148.

9) Weigend, F. Accurate Coulomb-Fitting Basis Sets for H to Rn. Phys. Chem. Chem. Phys. 2006, $8,1057-1065$.

10) CYLview, 1.0b, C.Y., Legault. Université de Sherbrooke, 2009 (http://www.cylview.org).

11) Tao, D.; Muuronen, M.; Slutskyy, Y.; Le, A.; Furche, F.; Overman, L. E. Diastereoselective Coupling of Chiral Acetonide Trisubstituted Radicals with Alkenes. Chem. Eur. J. 2016, 22, 8786-8790.

12) Nuhant, P.; Oderinde, M. S.; Genovino, J.; Juneau, A.; Gagné, Y.; Allais, C.; Chinigo, G. M.; Choi, C.; Sach, N. W.; Bernier, L.; Fobian, Y. M.; Bundesmann, M. W.; Khunte, B.; Frenette, M.; Fadeyi, O. O. Visible-Light-Initiated Manganese Catalysis for $\mathrm{C}-\mathrm{H}$ Alkylation of Heteroarenes: Applications and Mechanistic Studies. Angew. Chem. Int. Ed. 2017, 56, 1530915313.

13) Pitre, S. P.; McTiernan, C. D.; Vine, W.; DiPucchio, R.; Grenier, M.; Scaiano, J. C. VisibleLight Actinometry and Intermittent Illumination as Convenient Tools to Study $\mathrm{Ru}(\mathrm{bpy})_{3} \mathrm{Cl}_{2}$ Mediated Photoredox Transformations. Sci. Rep. 2015, 5, 16397.

14) Waser, J.; Gaspar, B.; Nambu, H.; Carreira, E. M. Hydrazines and Azides via the MetalCatalyzed Hydrohydrazination and Hydroazidation of Olefins. J. Am. Chem. Soc. 2006, 128, 11693-11712.

15) Lackner, G. L.; Quasdorf, K. W.; Overman, L. E. Direct Construction of Quaternary Carbons from Tertiary Alcohols via Photoredox-Catalyzed Fragmentation of tert-Alkyl $\mathrm{N}$ Phthalimidoyl Oxalates J. Am. Chem. Soc. 2013, 135, 15342-15345. 
16) Bristol-Myers Squibb Company; Hiebert, S.; Rajamani, R.; Sun, L.; Mull, E.; Gillis, E. P.; Bowsher, M. S.; Zhao, Q.; Meanwell, N. A.; Renduchintala, K. V.; Sarkunam, K.; Nagalakshmi, P.; Babu, P. V. K. S.; Scola, P. M. Preparation of Macrocyclic Peptides, Especially Proline-Containing Peptides, as Inhibitors of Hepatitis C Virus Replication. US Patent US 2013115190, 2013.

17) Bristol-Myers Squibb Company; Balog, J. A.; Cherney, E. C.; Guo, W.; Huang, A.; Markwalder, J. A.; Seitz, S. P.; Shan, W.; Williams, D. K.; Murugesan, N.; Nara, S. J.; Roy, S.; Thangavel, S.; Sistla, R. K.; Cheruku, S.; Thangathirupathy, S.; Kanyaboina, Y.; Pulicharla, N. Preparation of Benzenediamine Derivatives as Inhibitors of Indoleamine 2,3-dioxygenase for the Treatment of Cancer. US Patent WO 2016161269, 2016.

18) Fan, C.; Zhu, L.; Jiang, B.; Zhong, C.; Ma, D.; Qin, J.; Yang, C. Side Chain Effect on Photovoltaic Properties of D-A Copolymers Based on Benzodithiophene and ThiopheneSubstituted Bithiazole. Org. Electron. 2013, 14, 3162-3171.

19) Buffel, D. K.; McGuigan, C.; Robins, M. J. Nucleic acid related compounds. 48. Photoaddition of methanol to $9-(\beta-D$-ribofuranosyl) purine (nebularine) to give inhibitors of adenosine deaminase J. Org. Chem. 1985, 50, 2664-2667.

20) Less than $5 \%$ of regioisomeric product was detected by NMR analysis of the crude reaction mixture, or by UV analysis during purification of the crude product (Biotage Isolera One). 


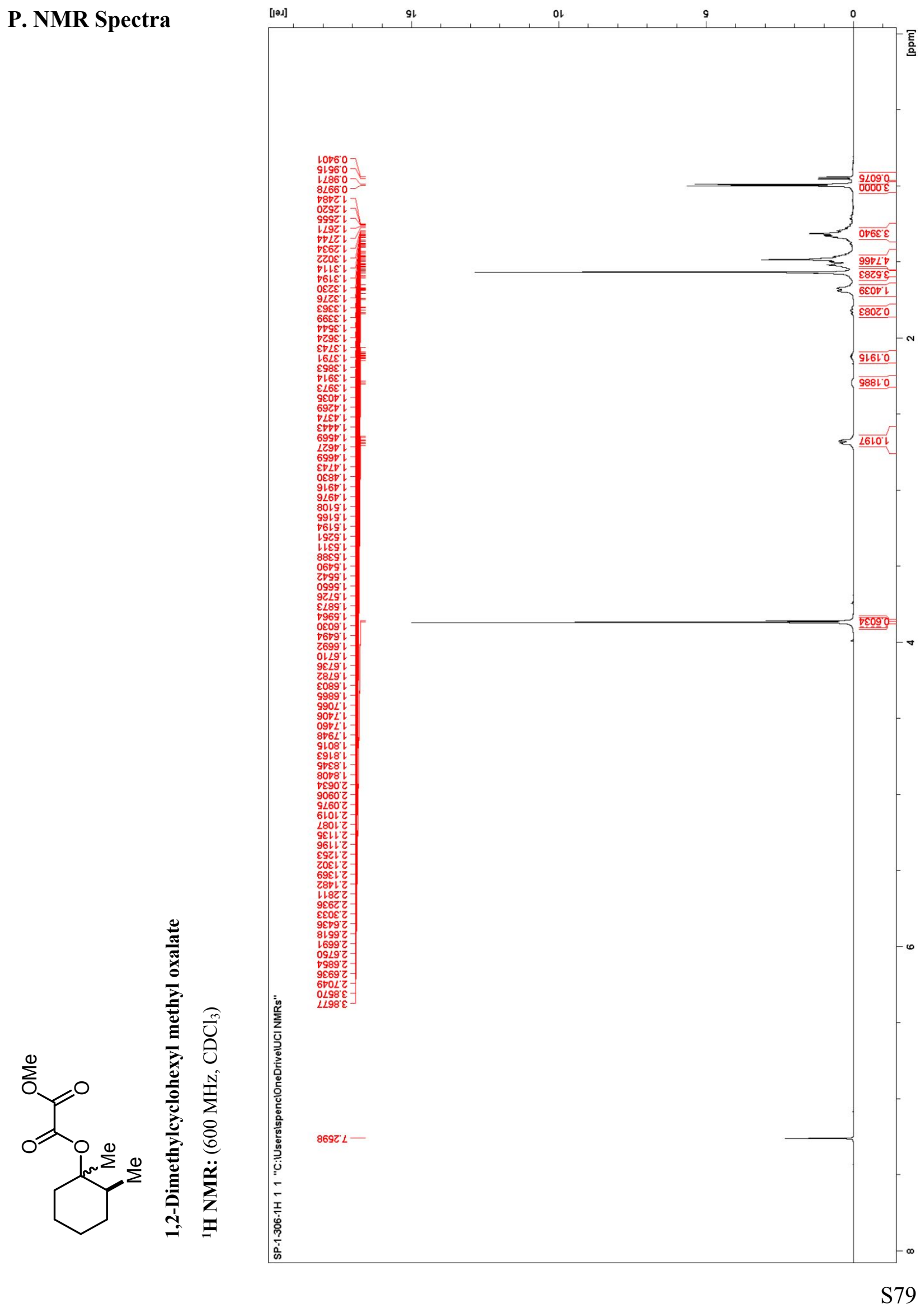




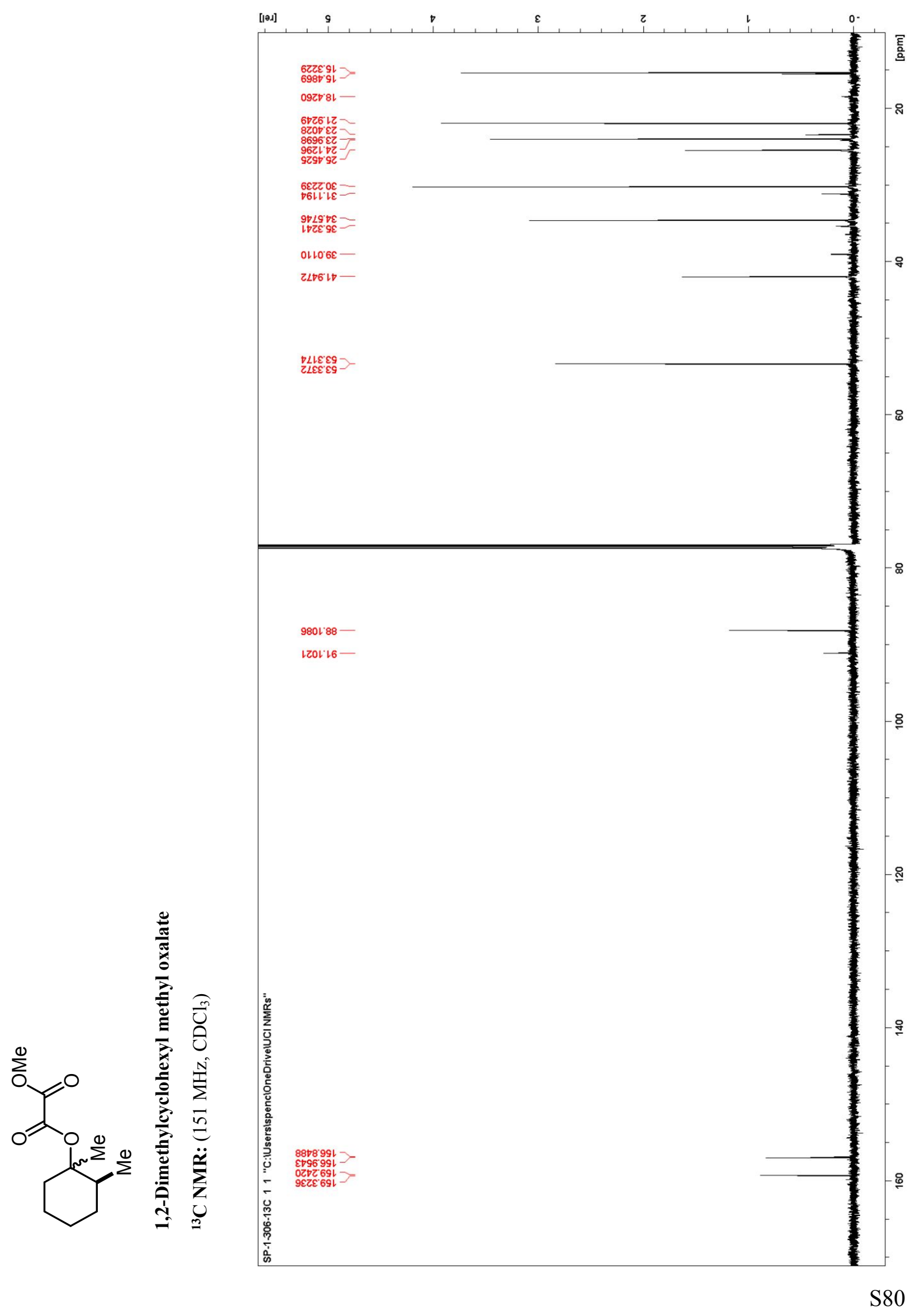




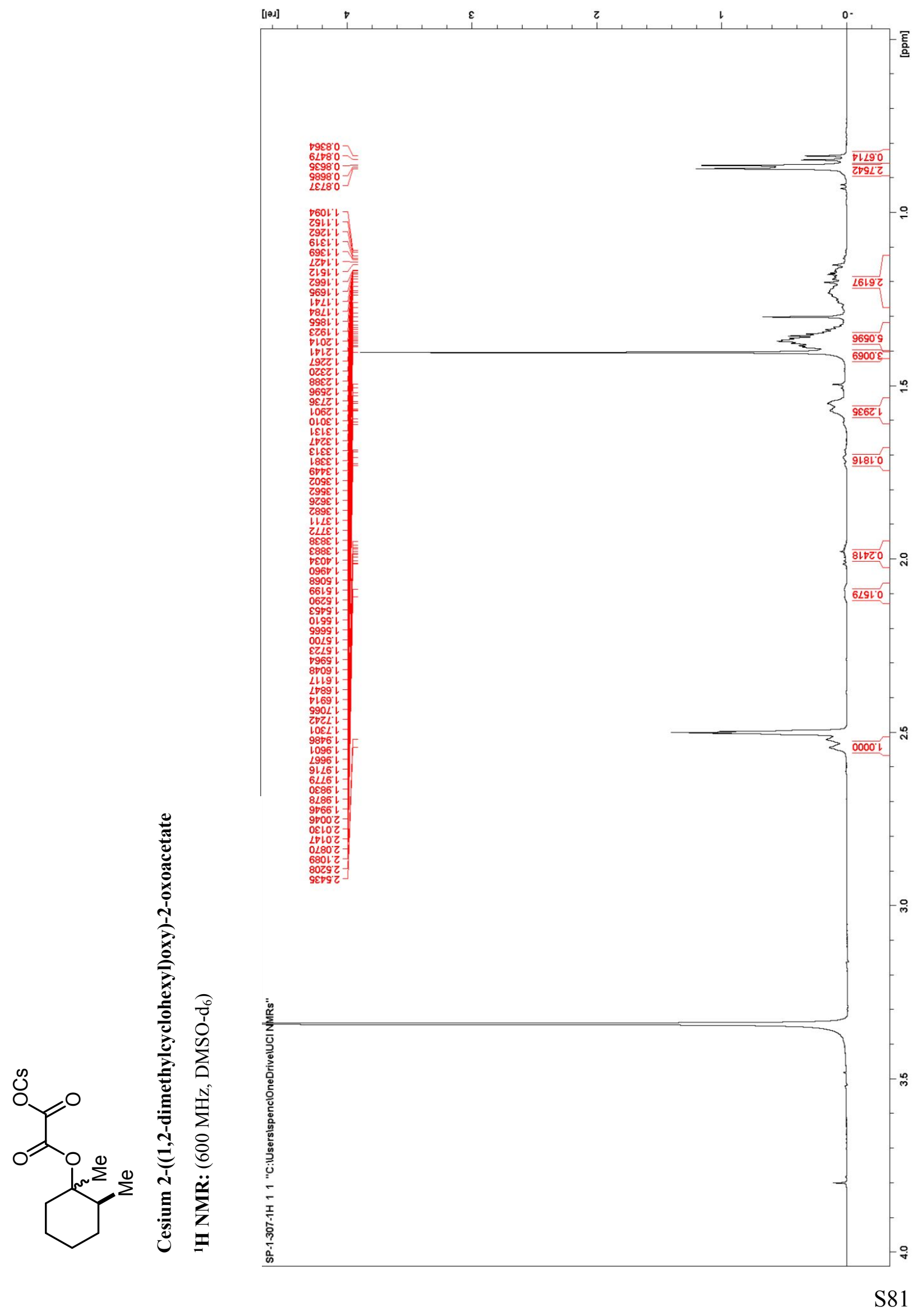




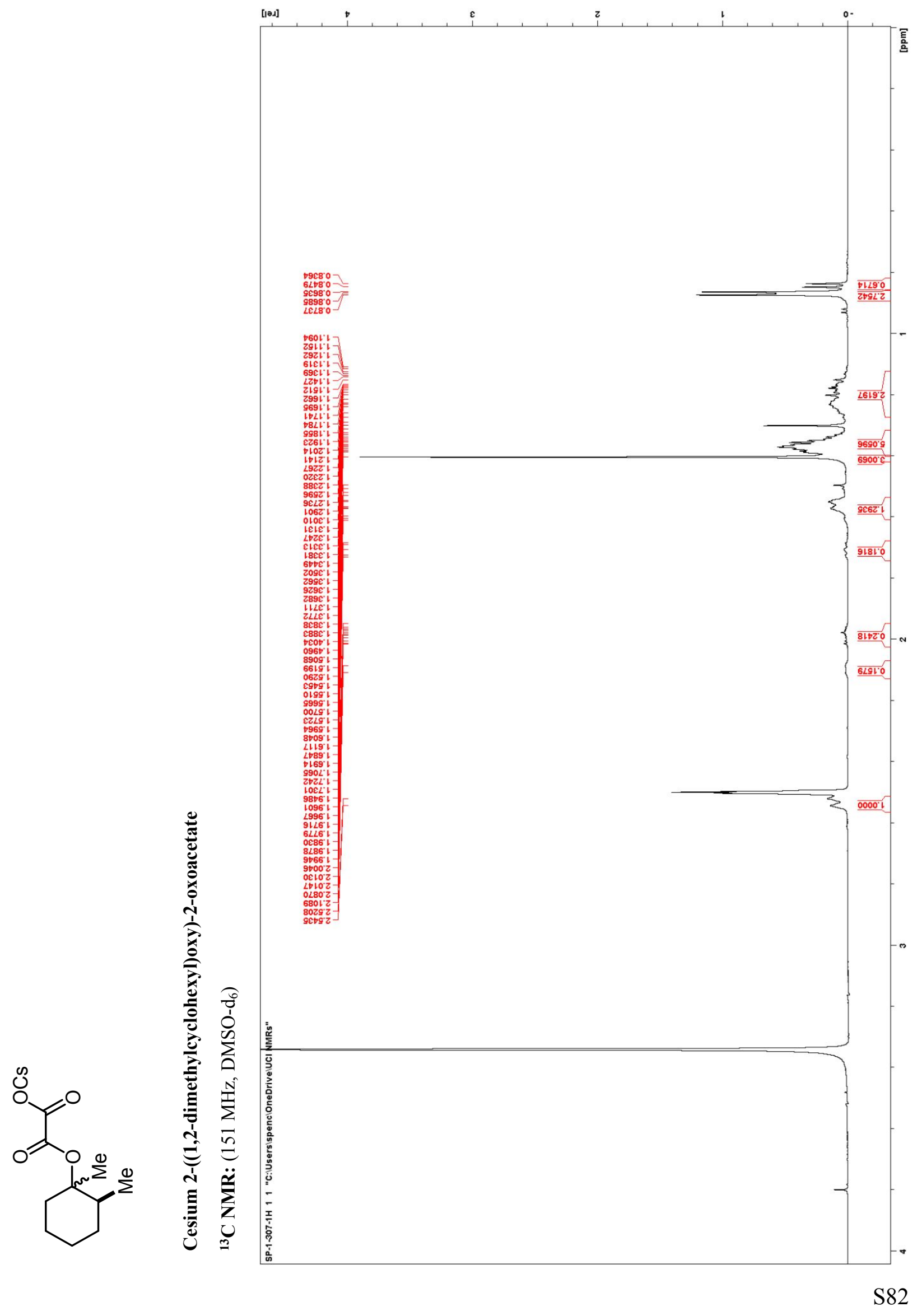




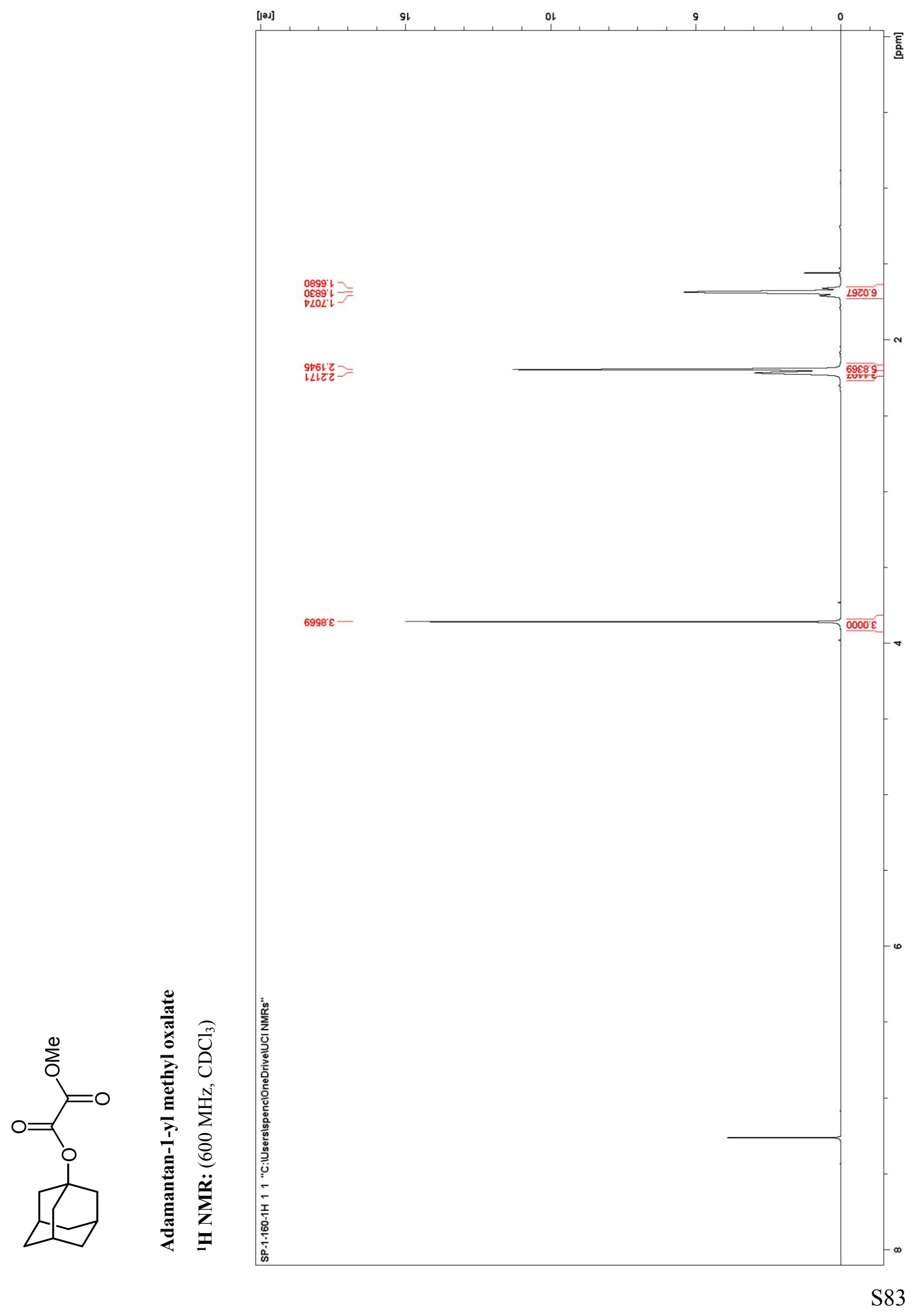




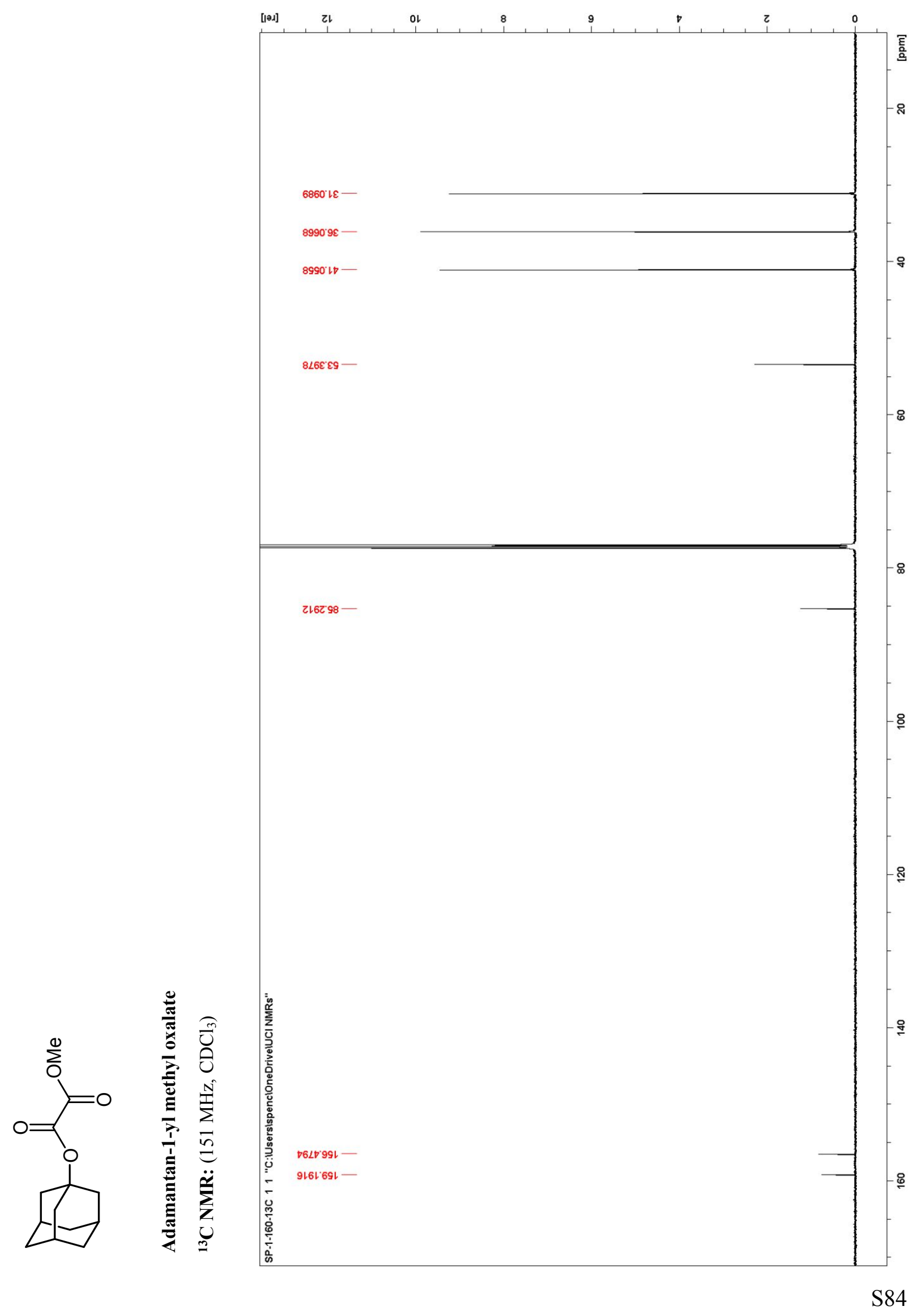




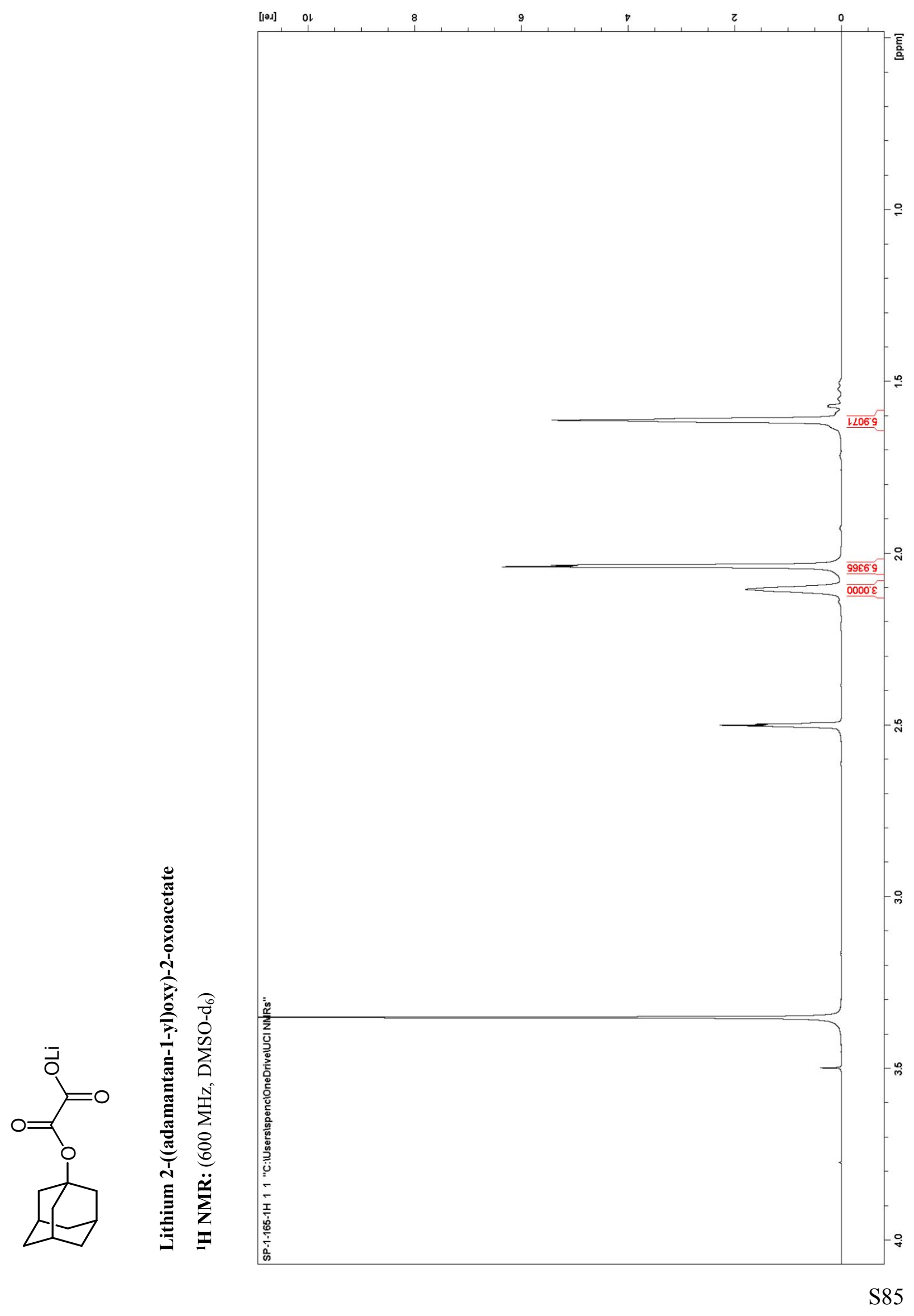




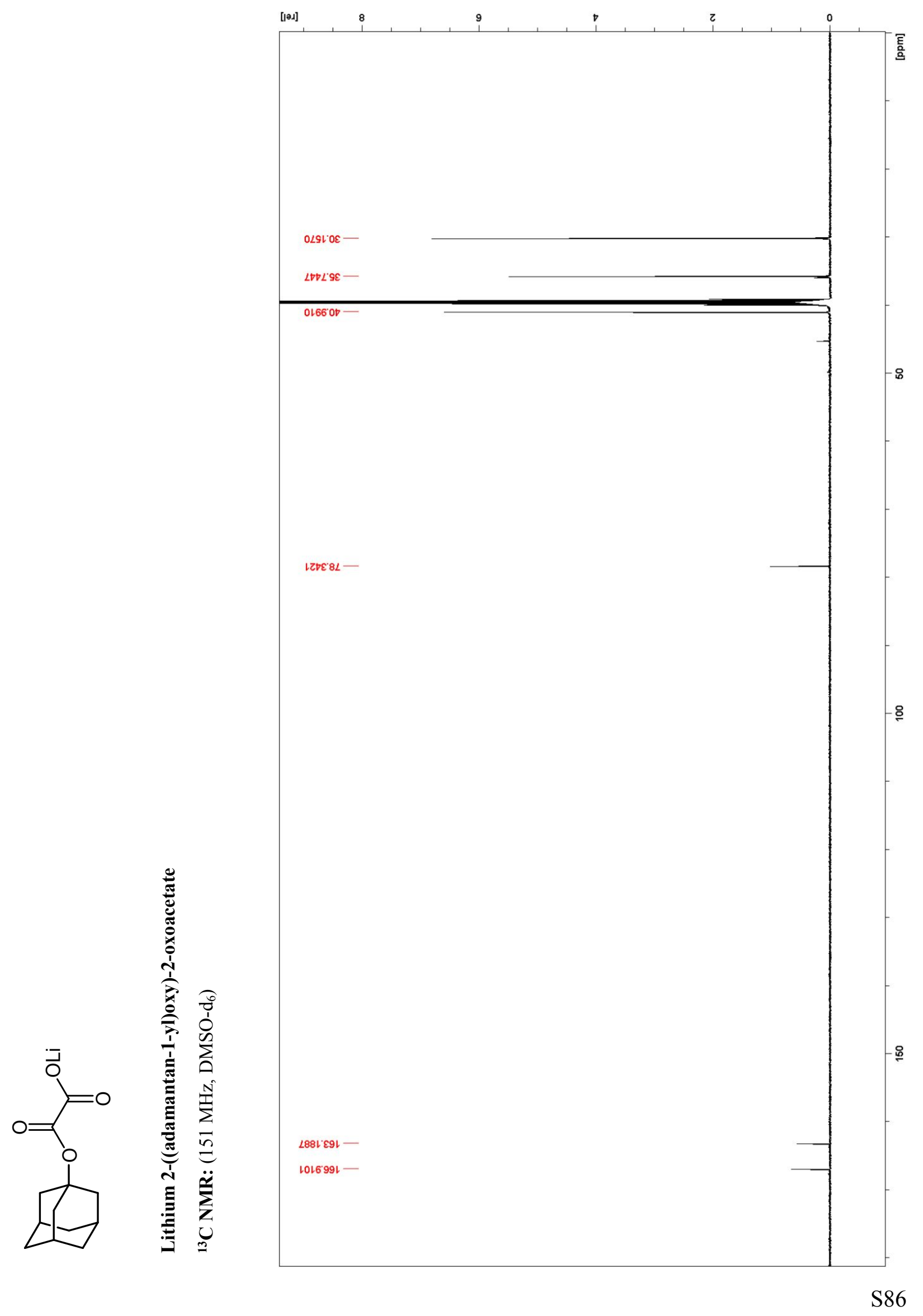




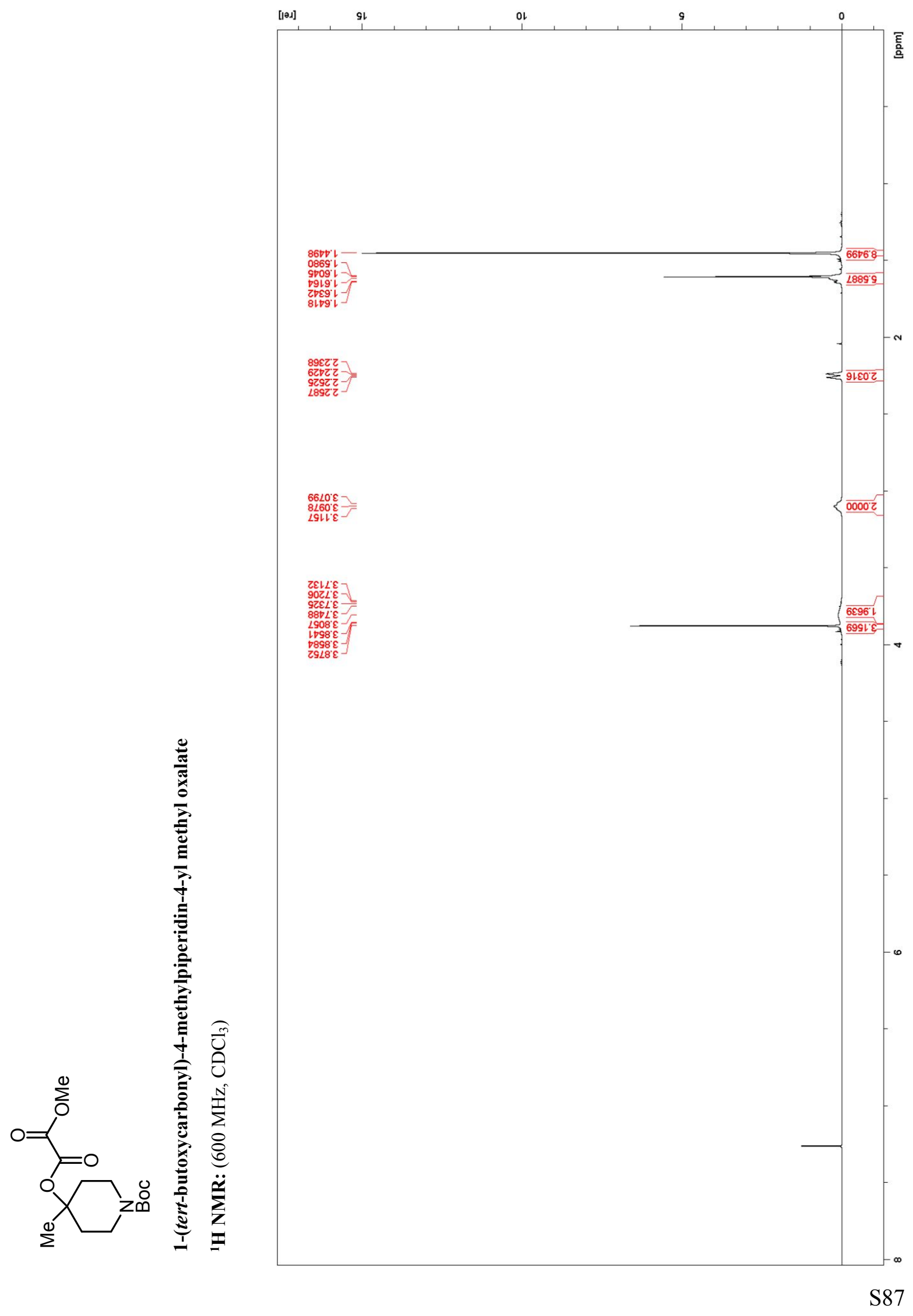




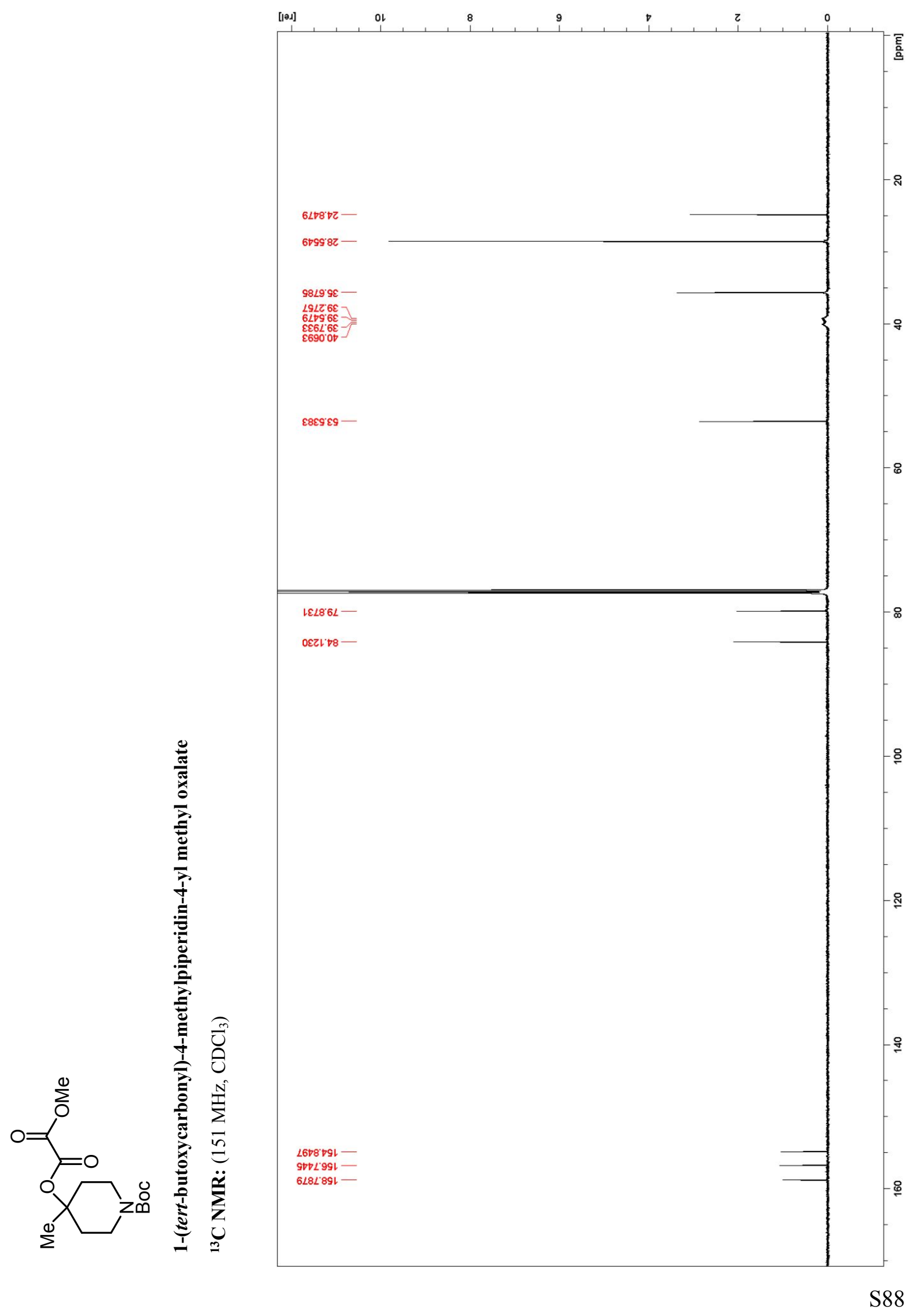




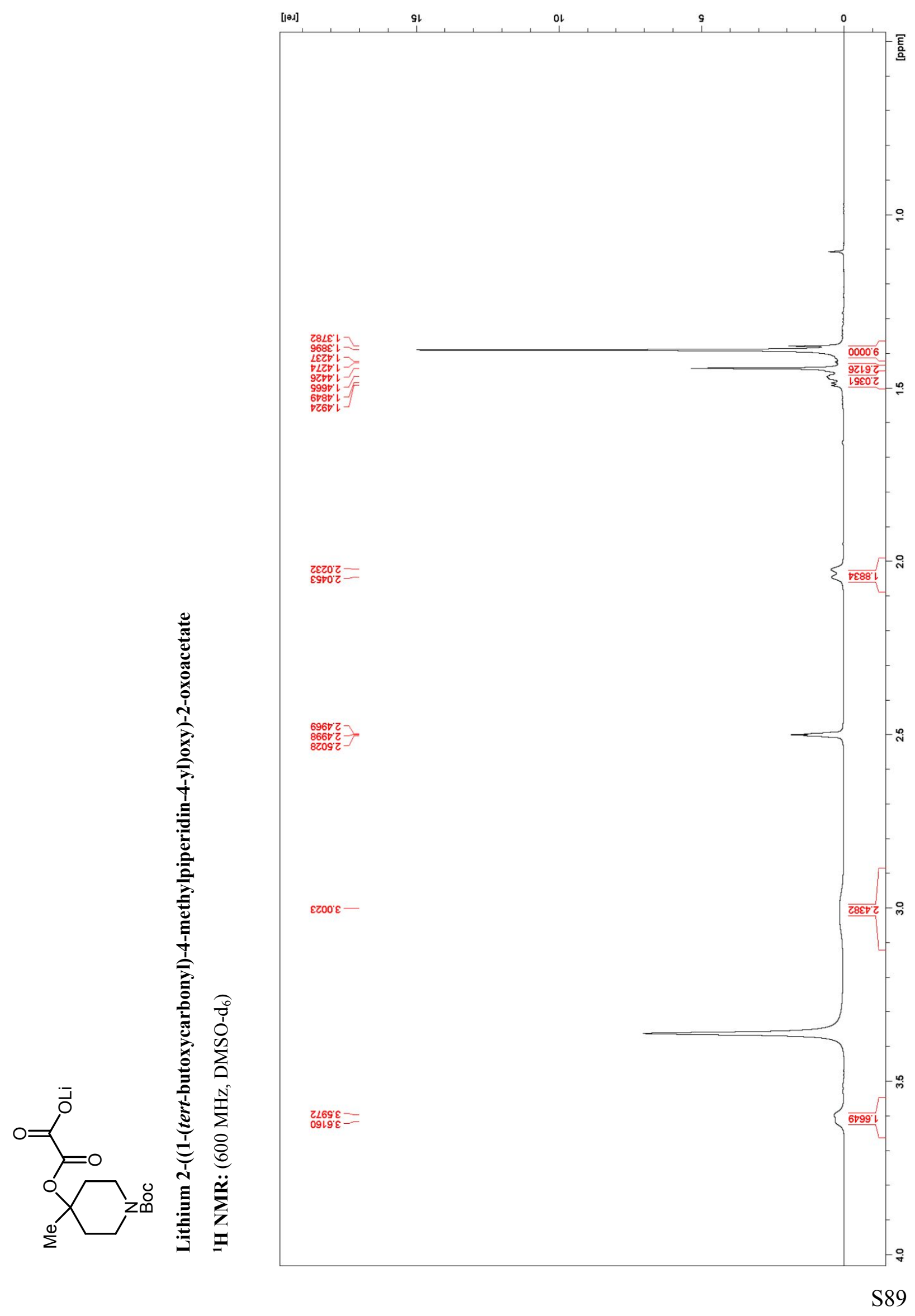




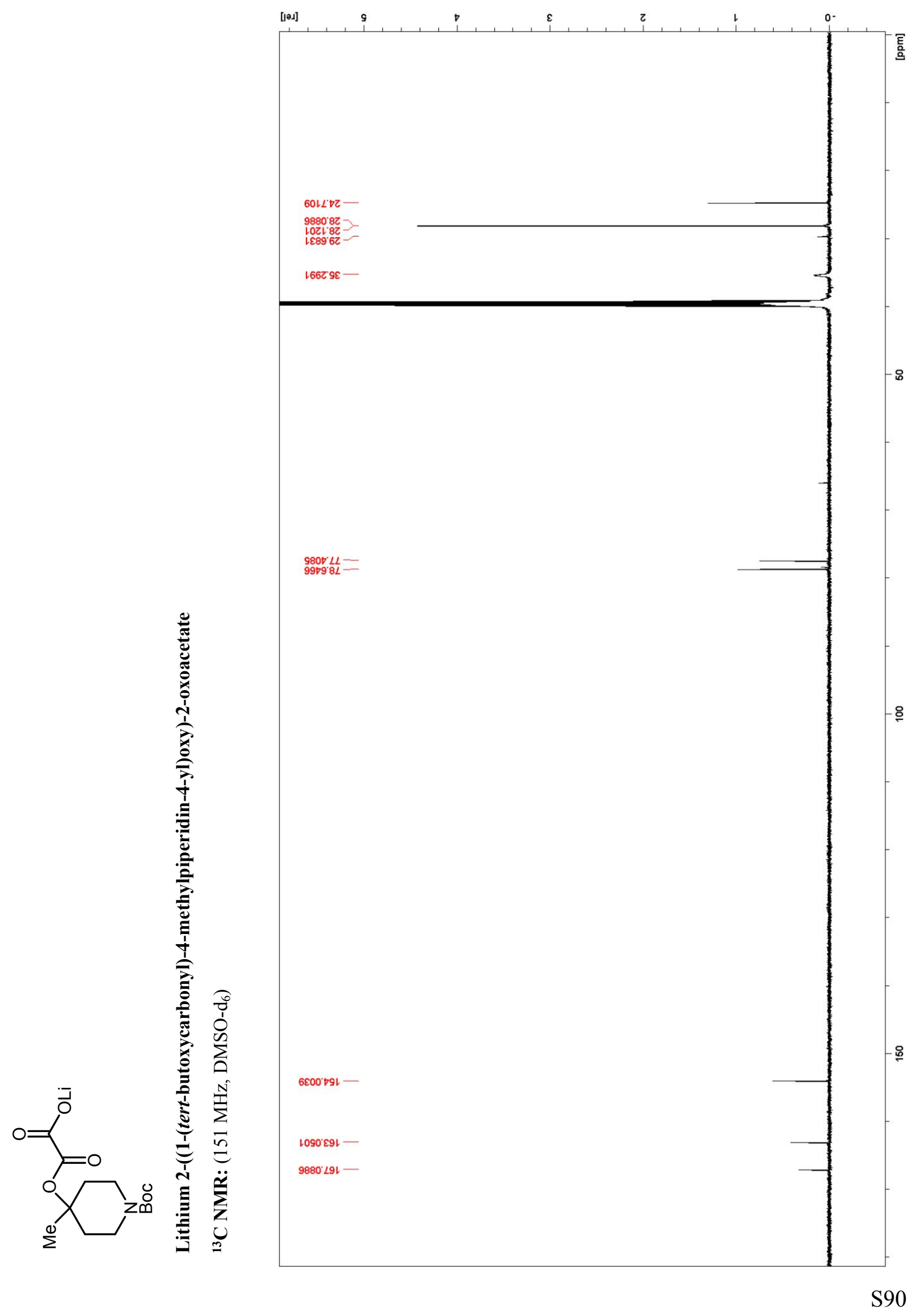




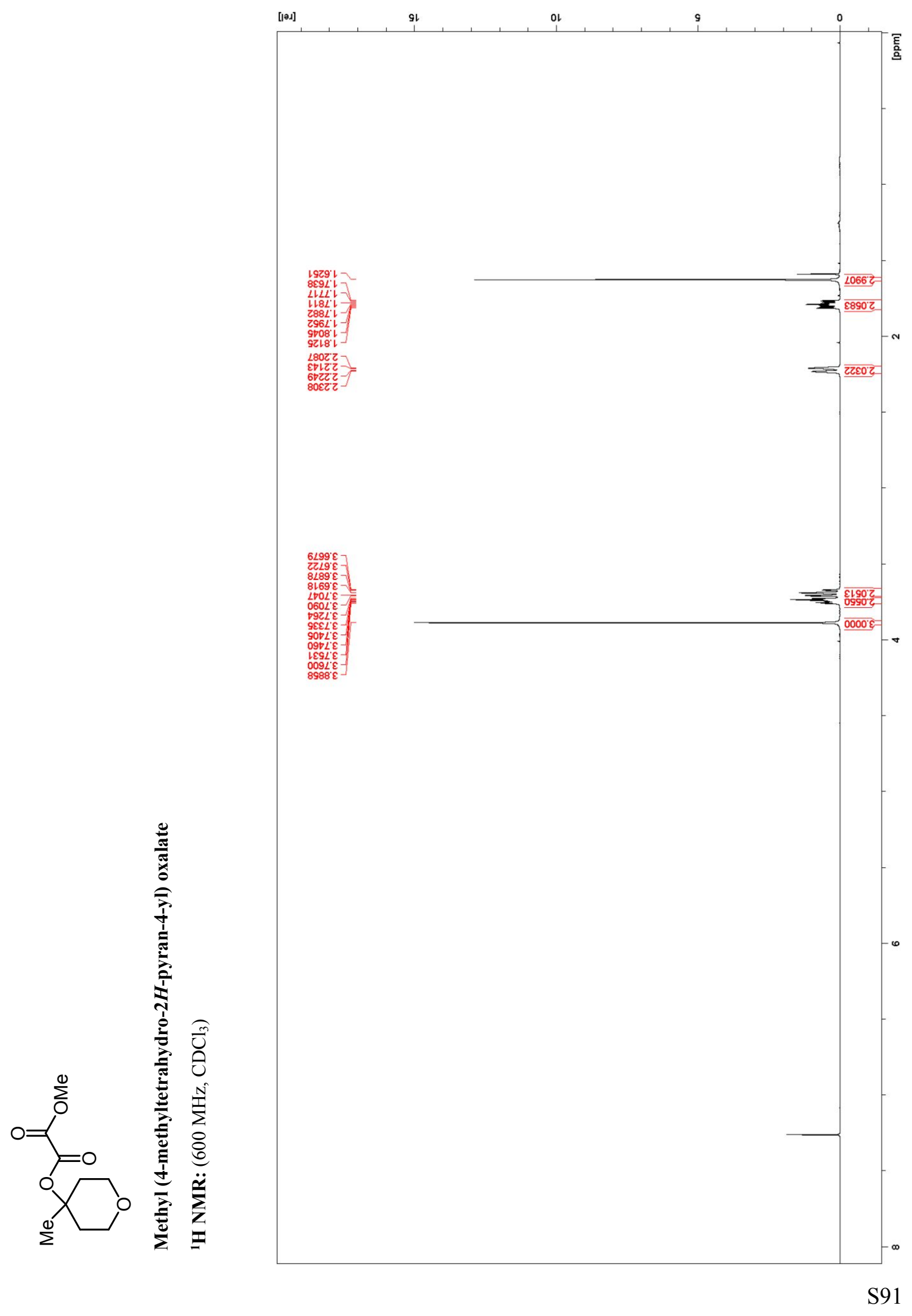




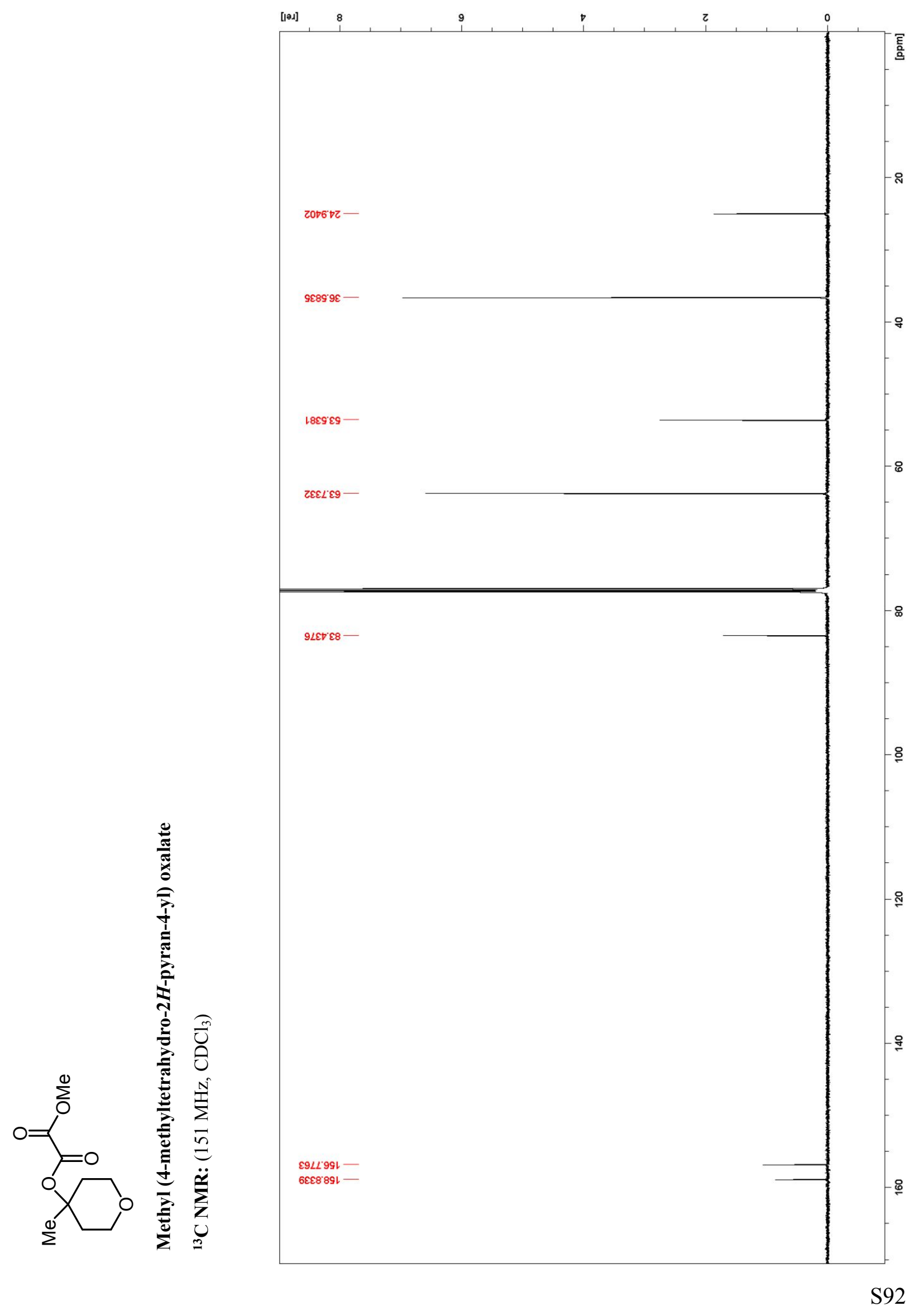




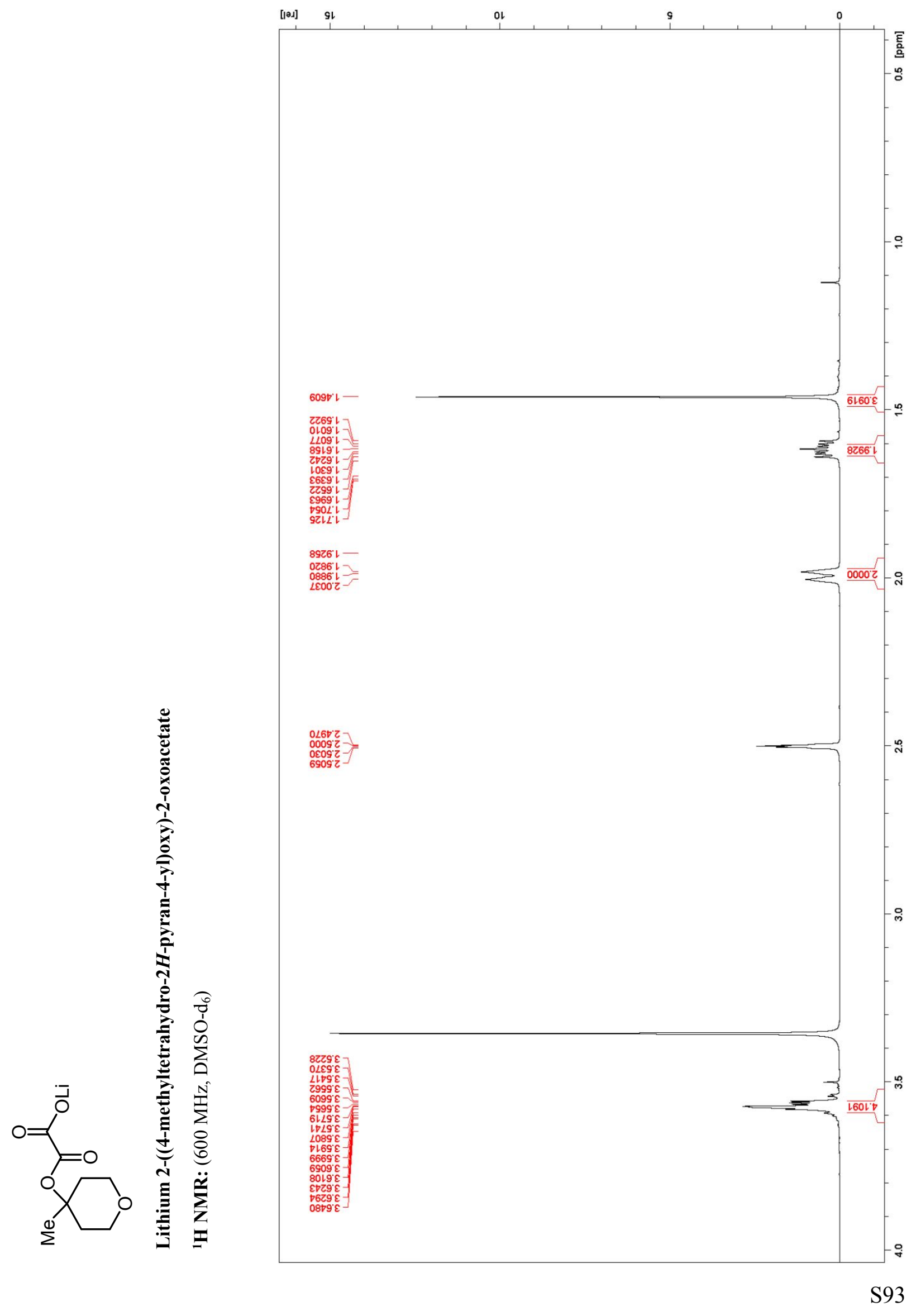




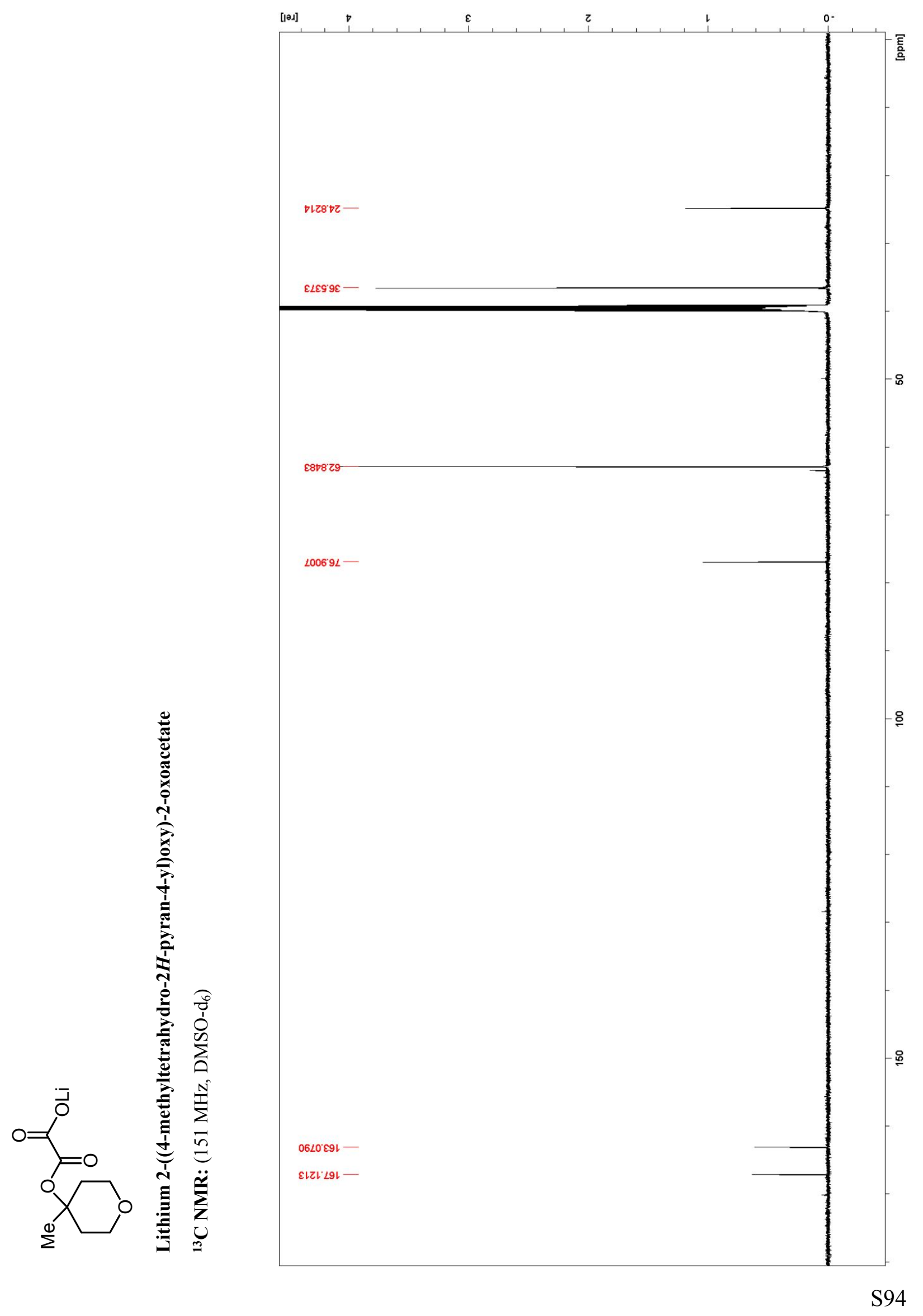




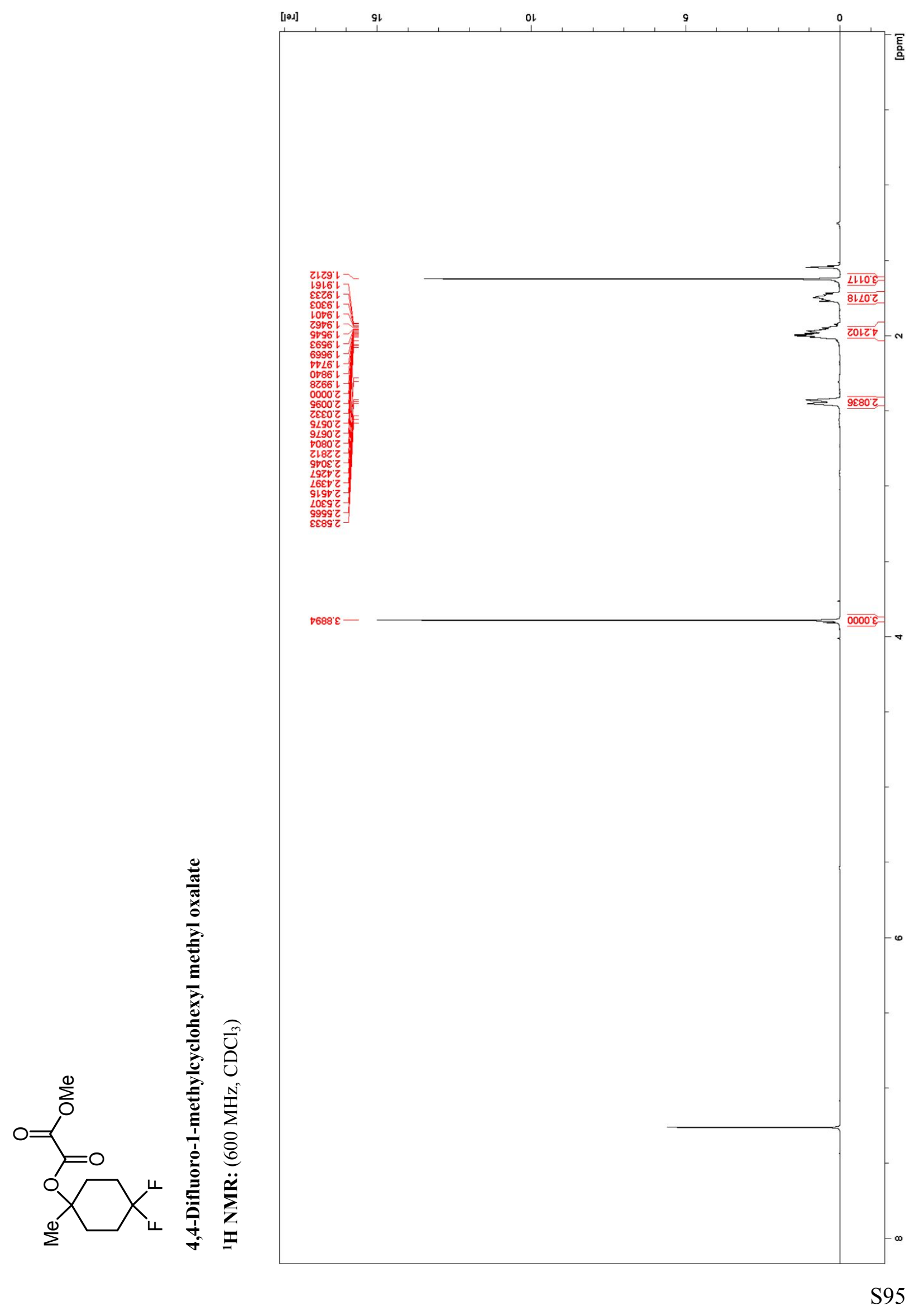




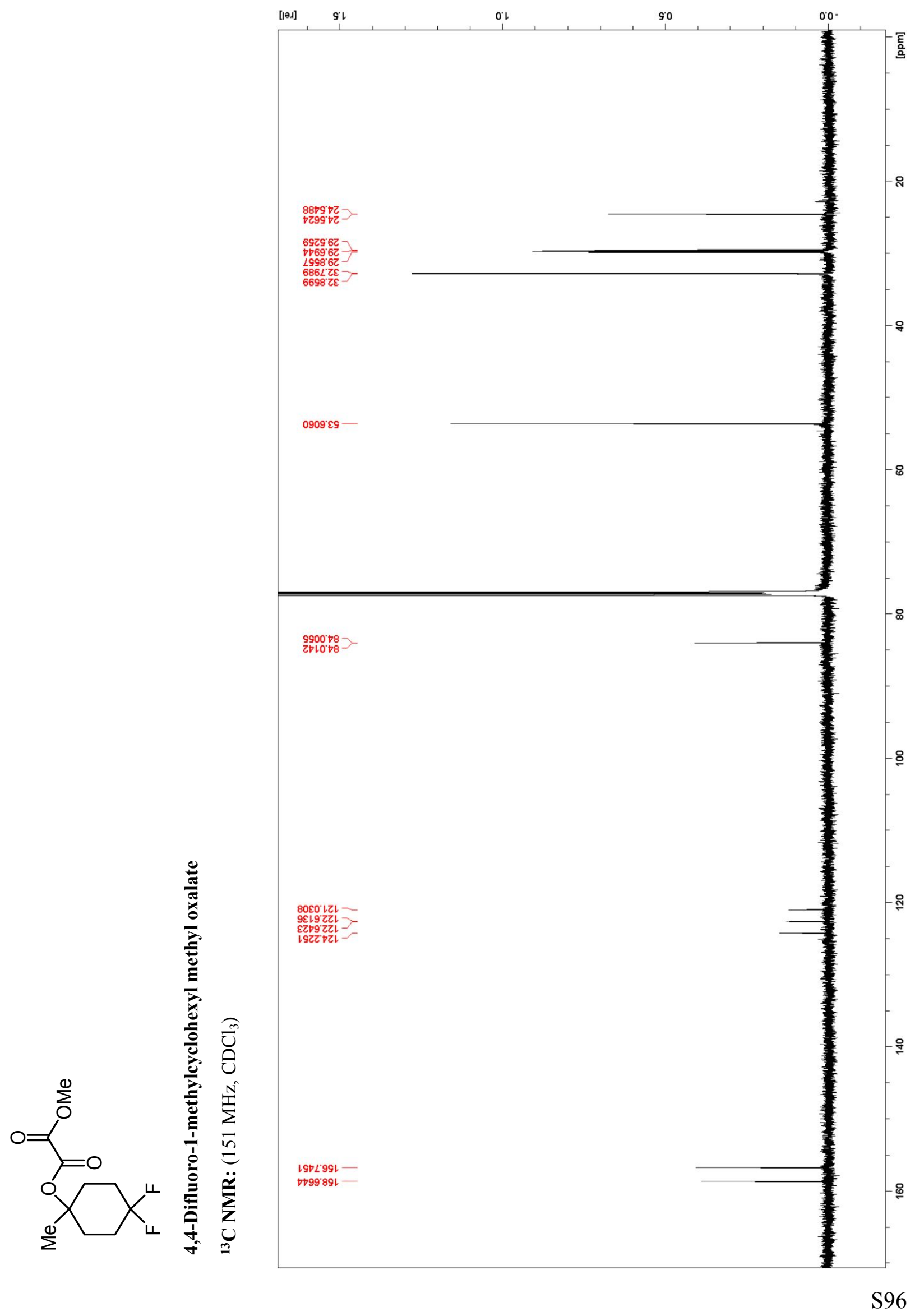




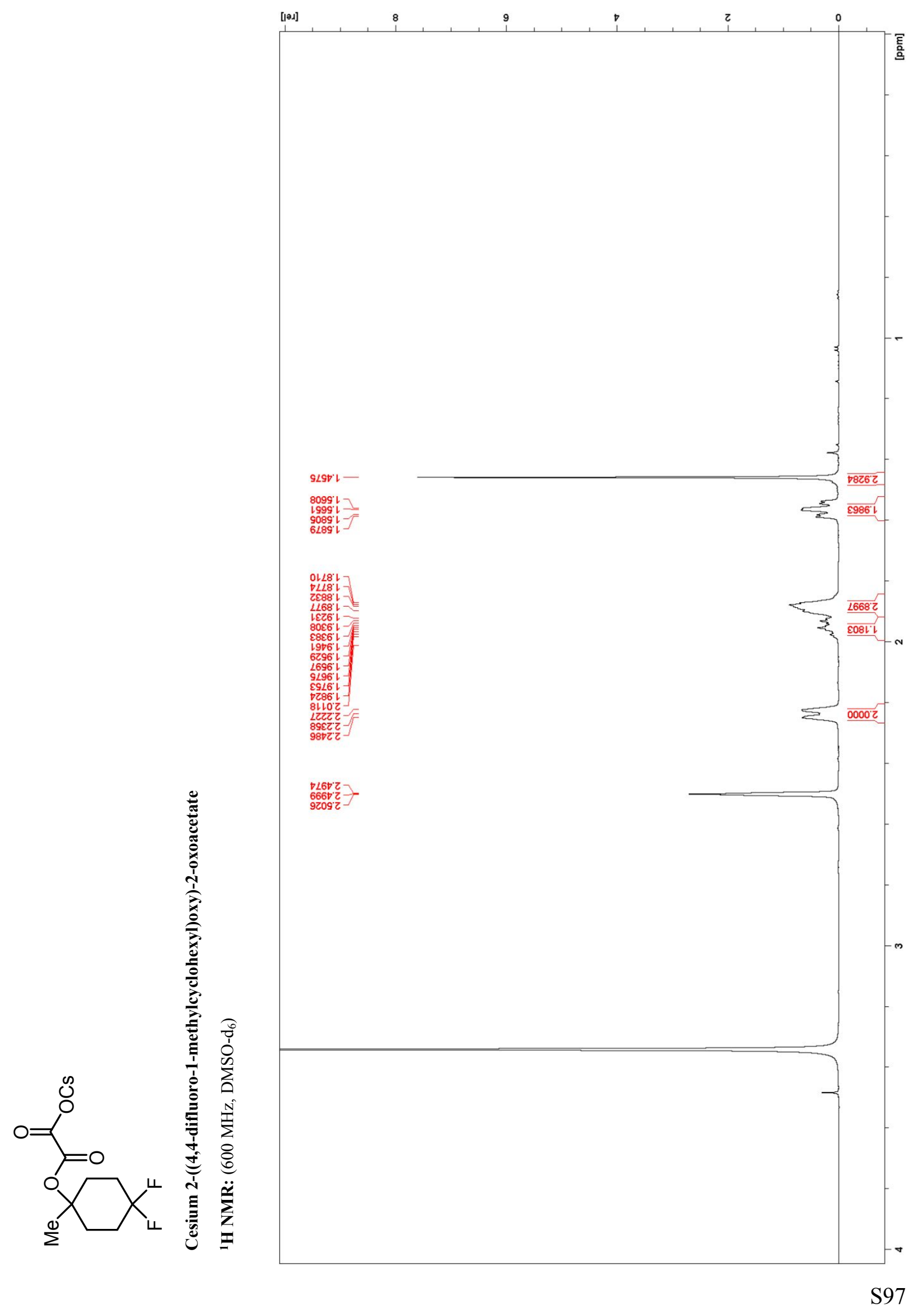




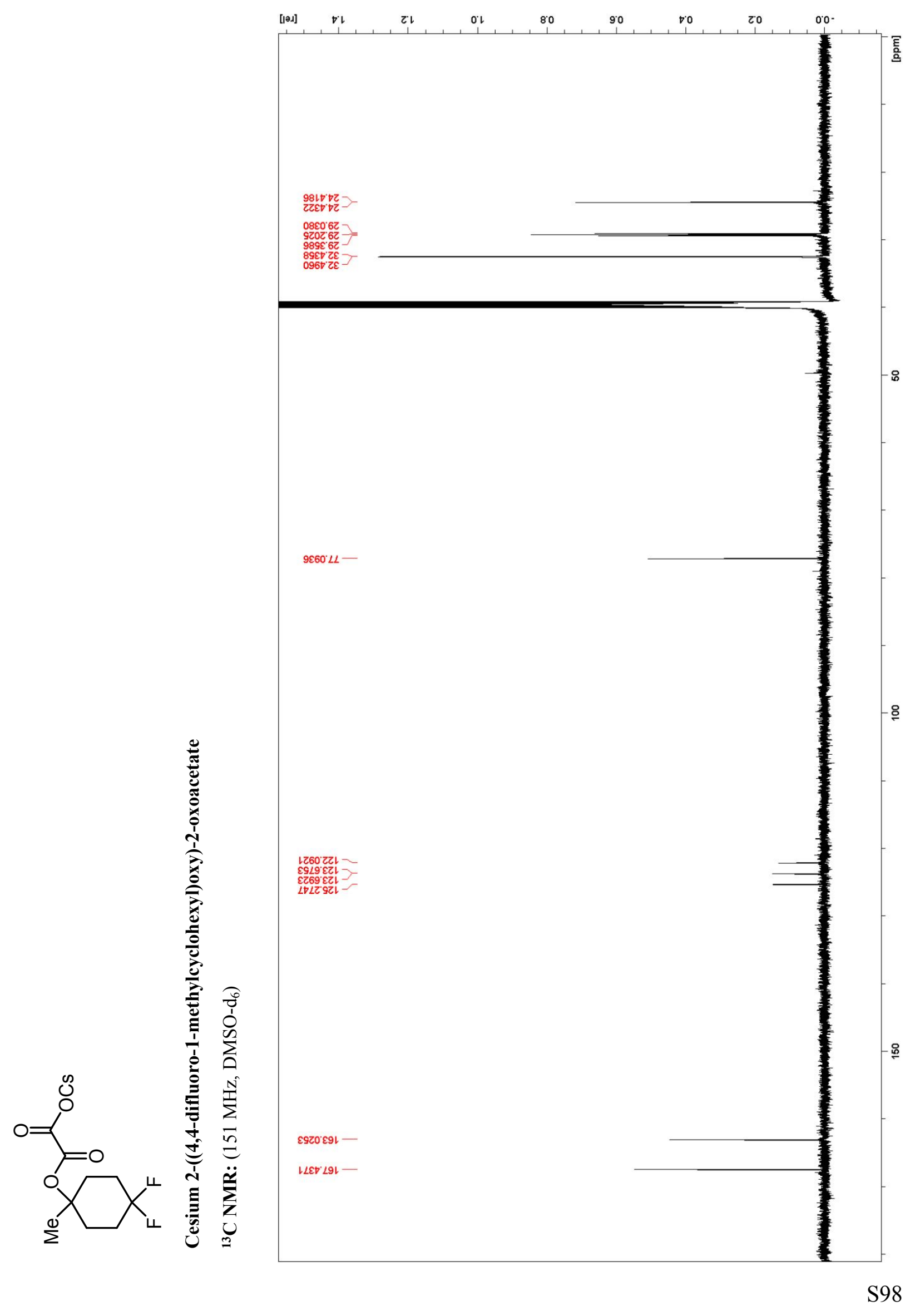




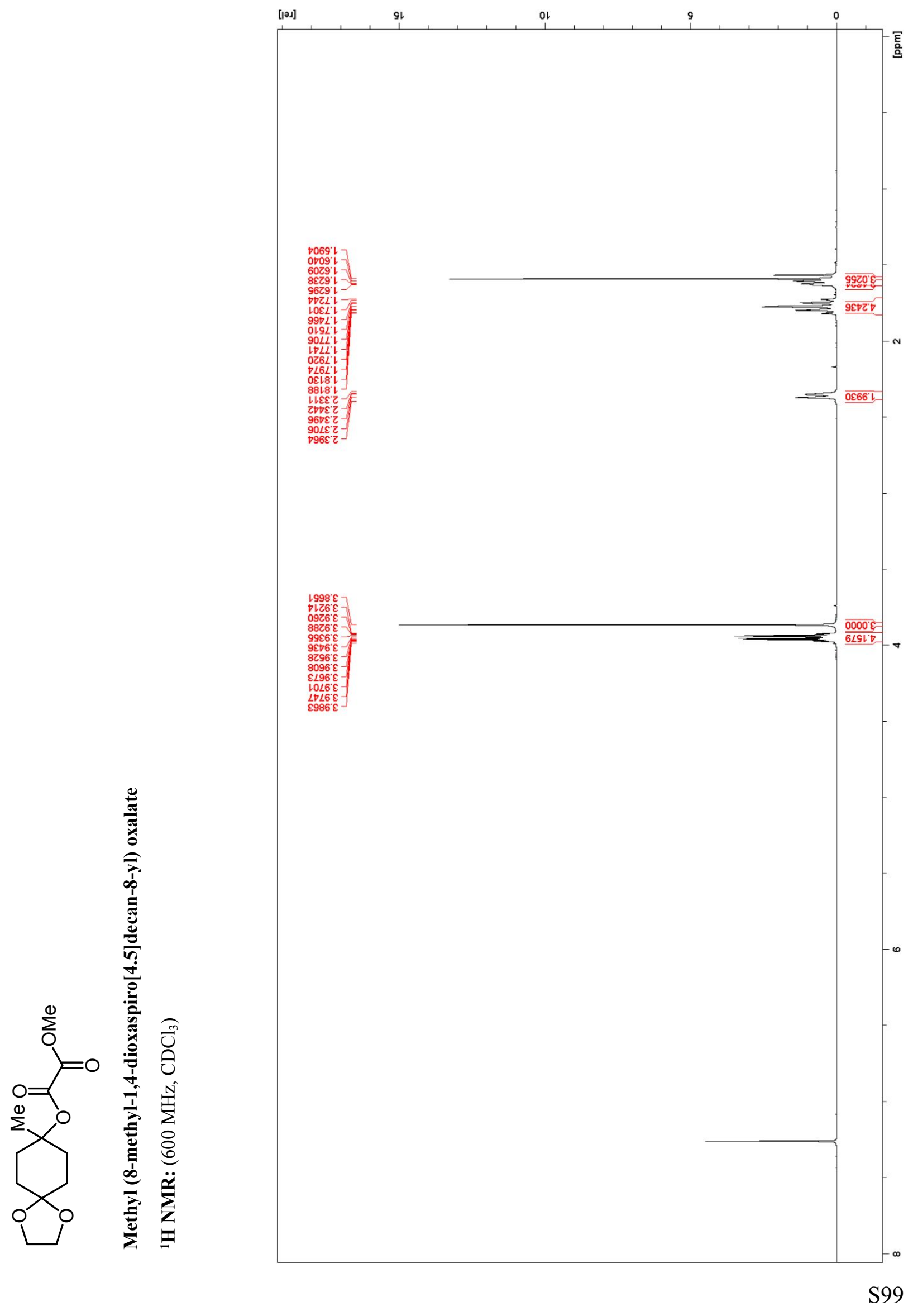




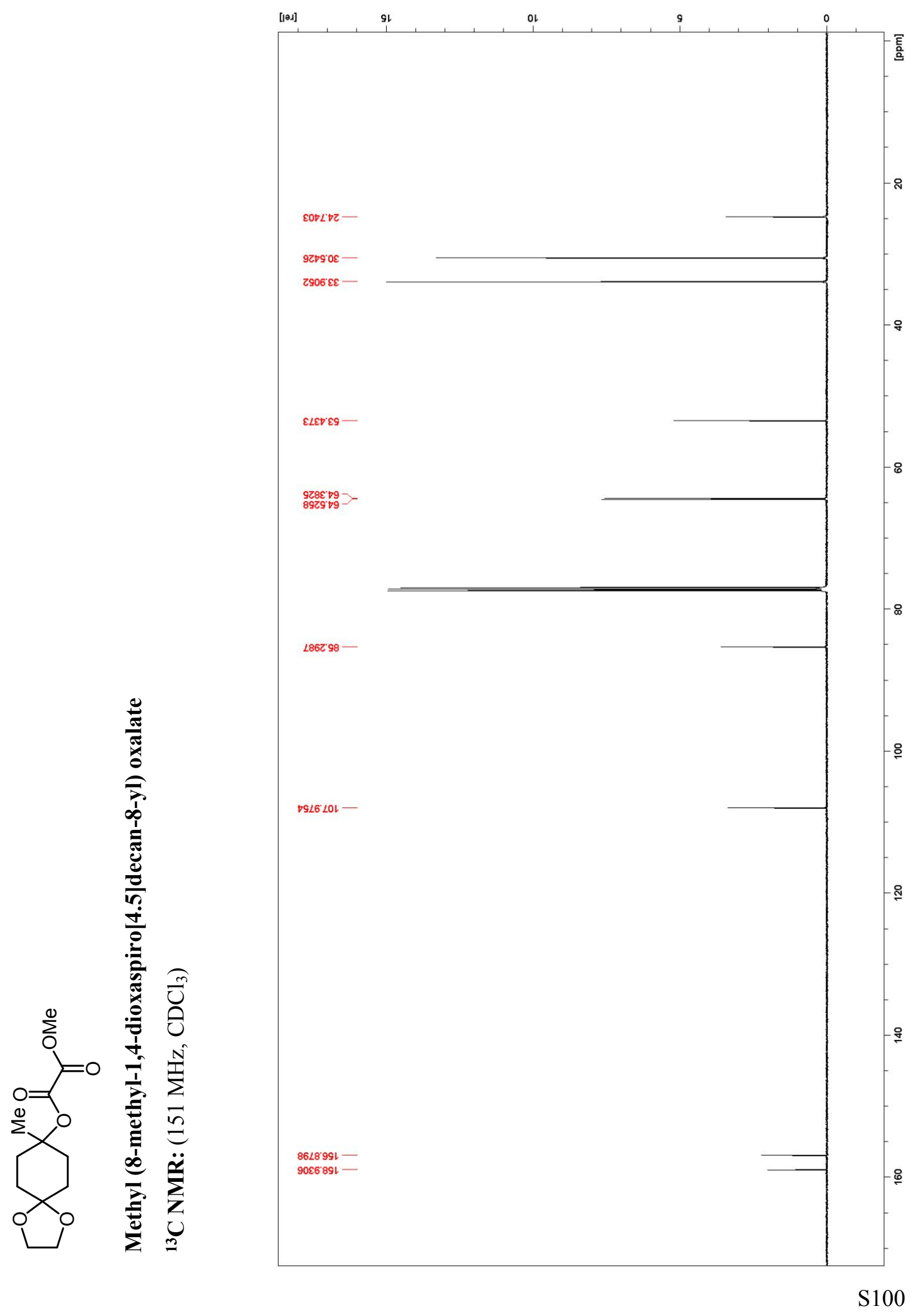




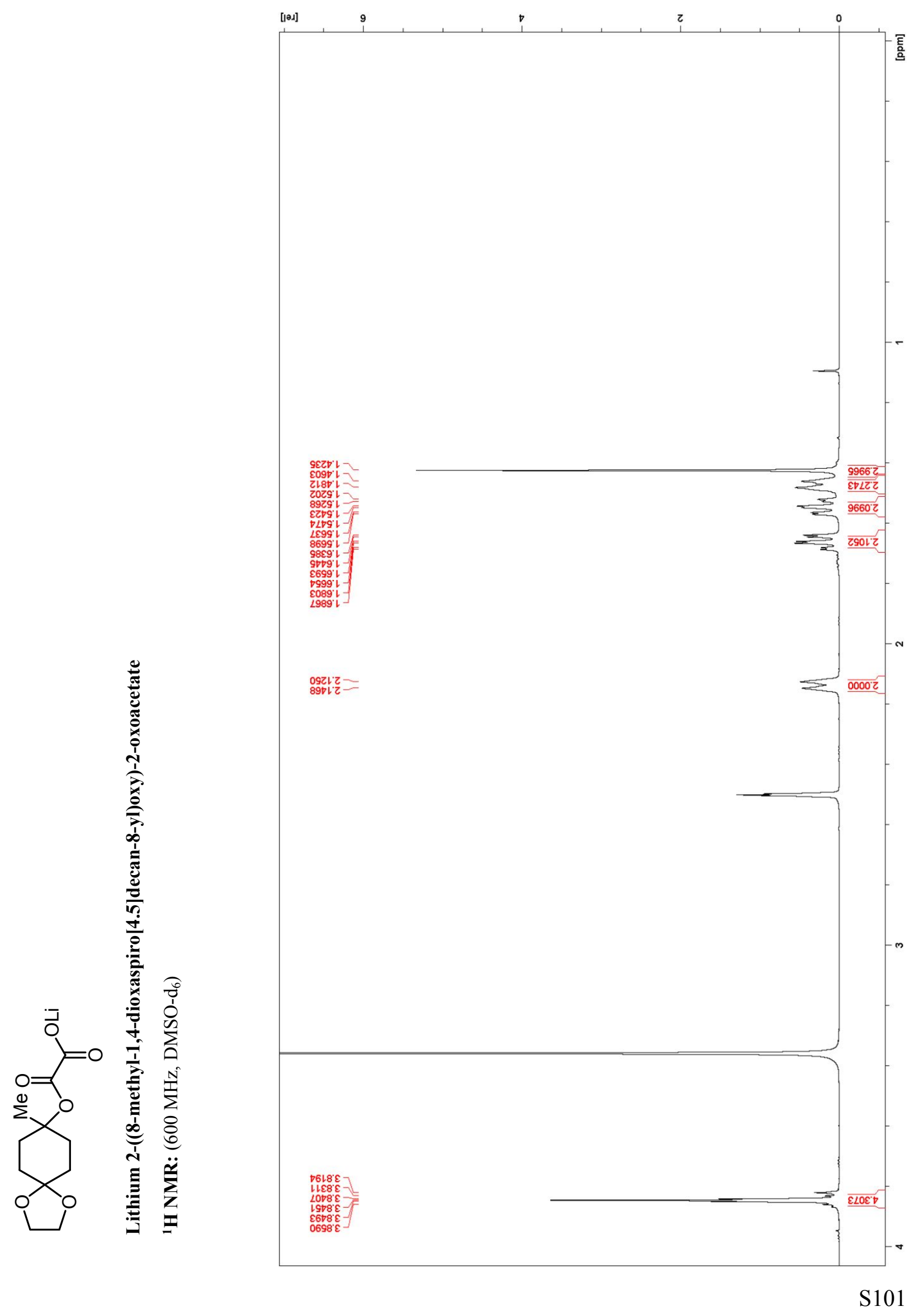




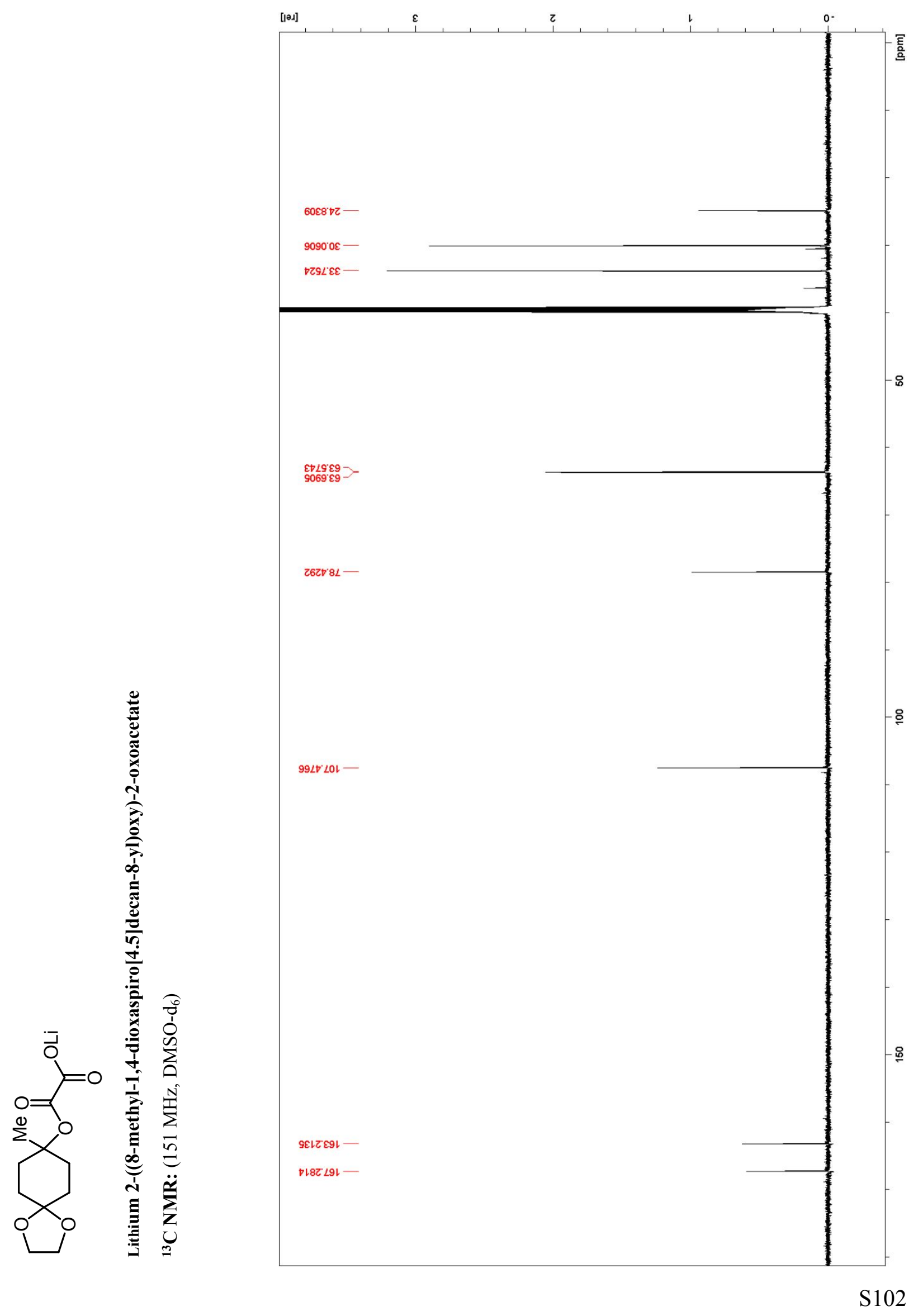




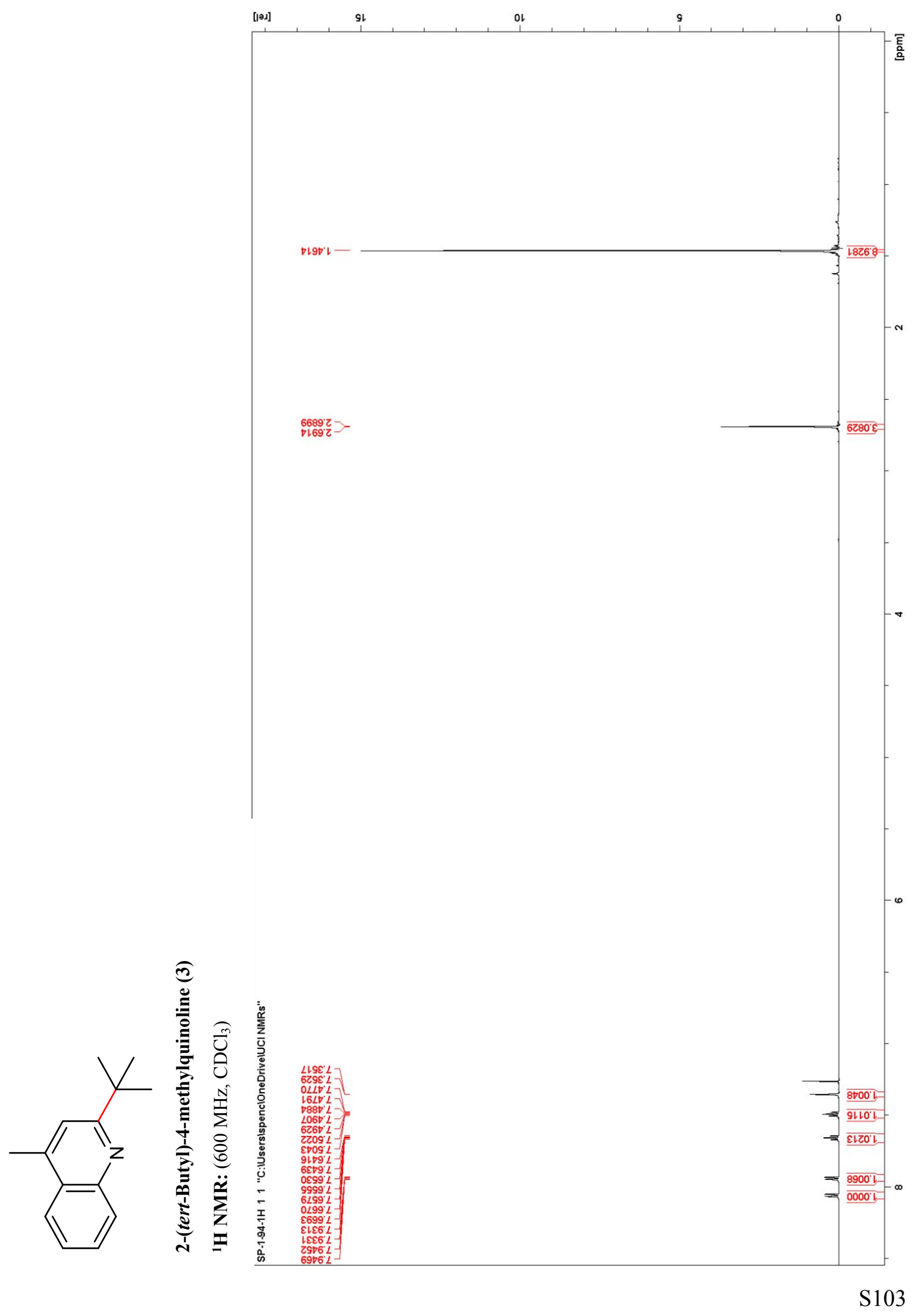




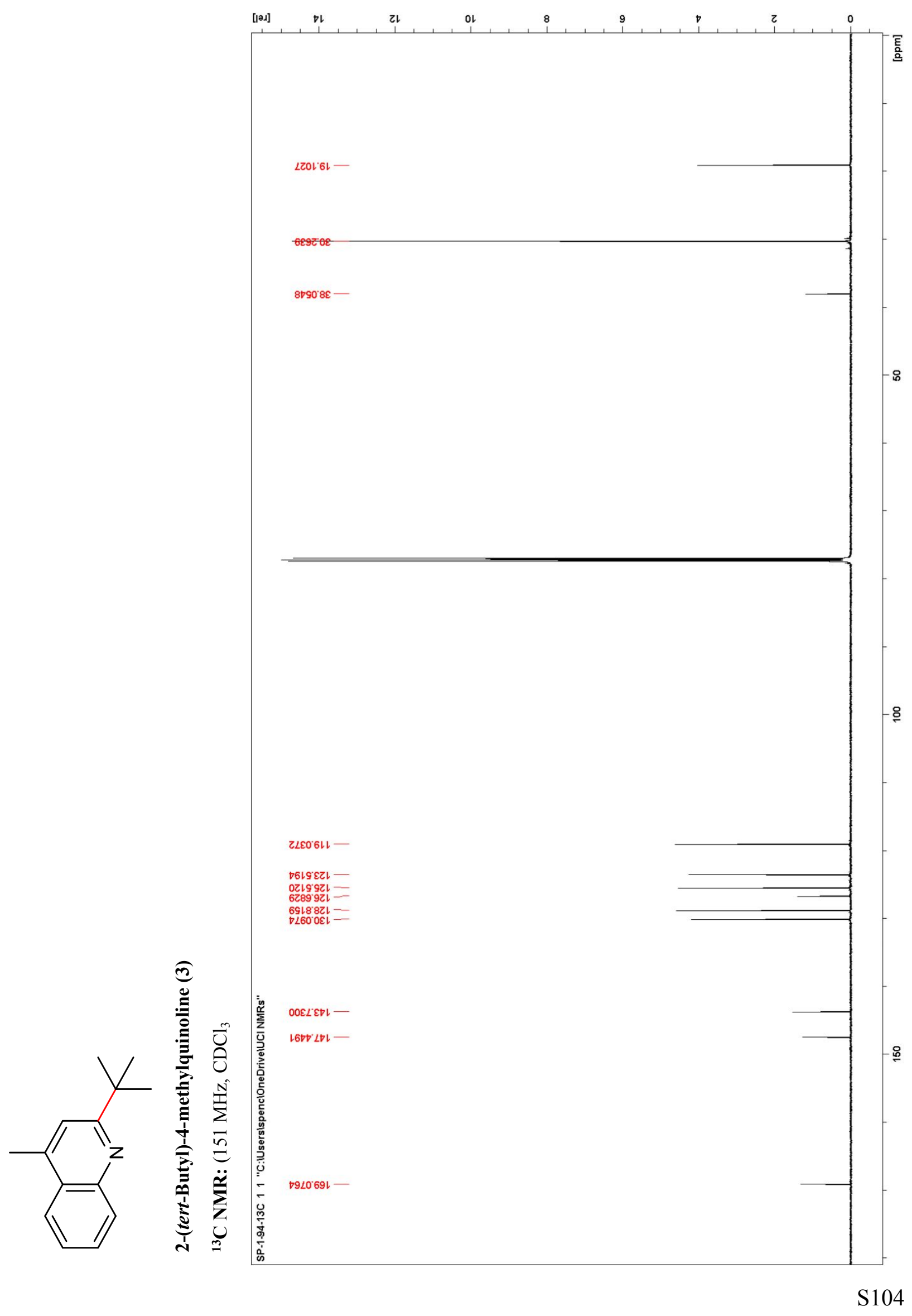




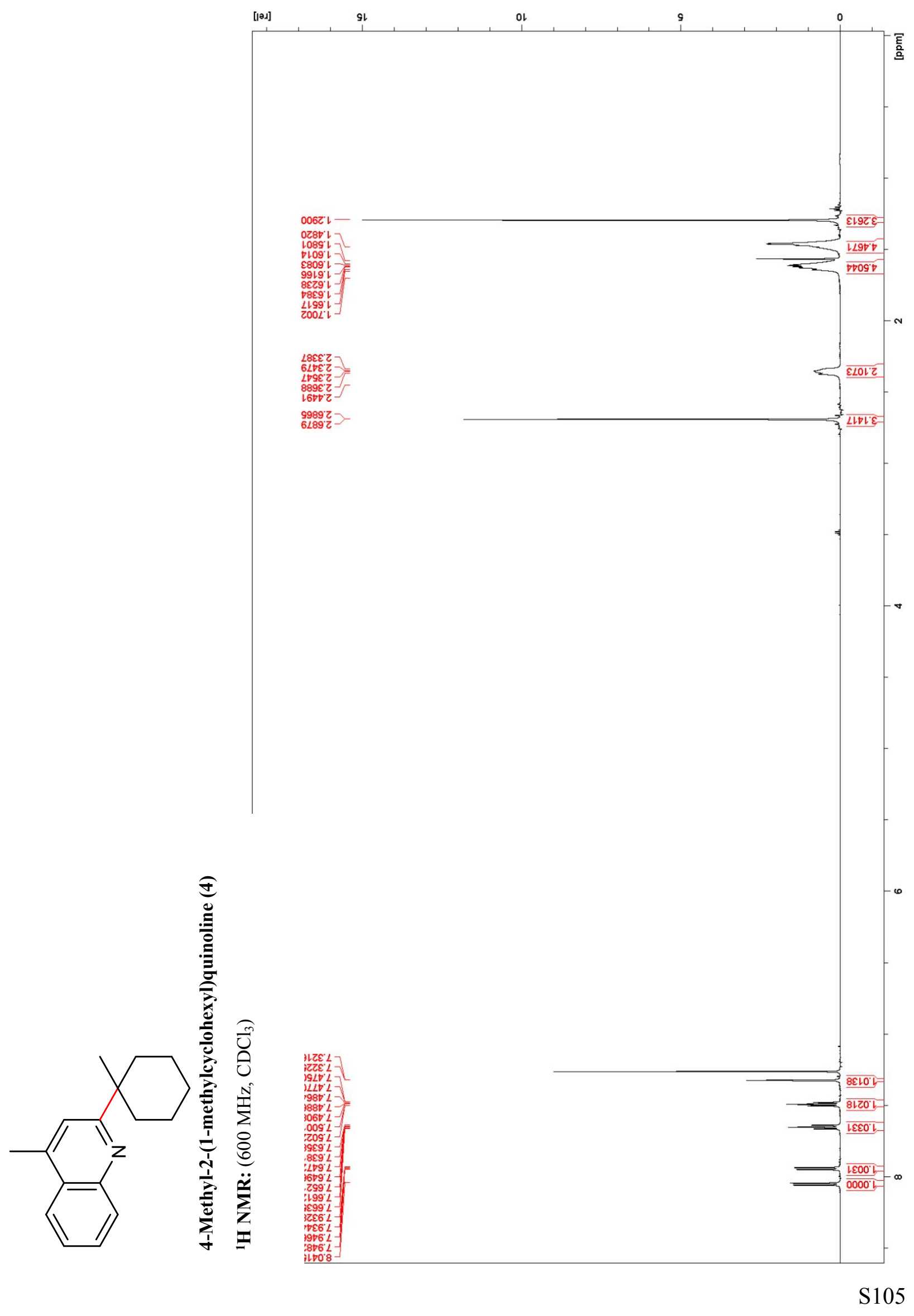




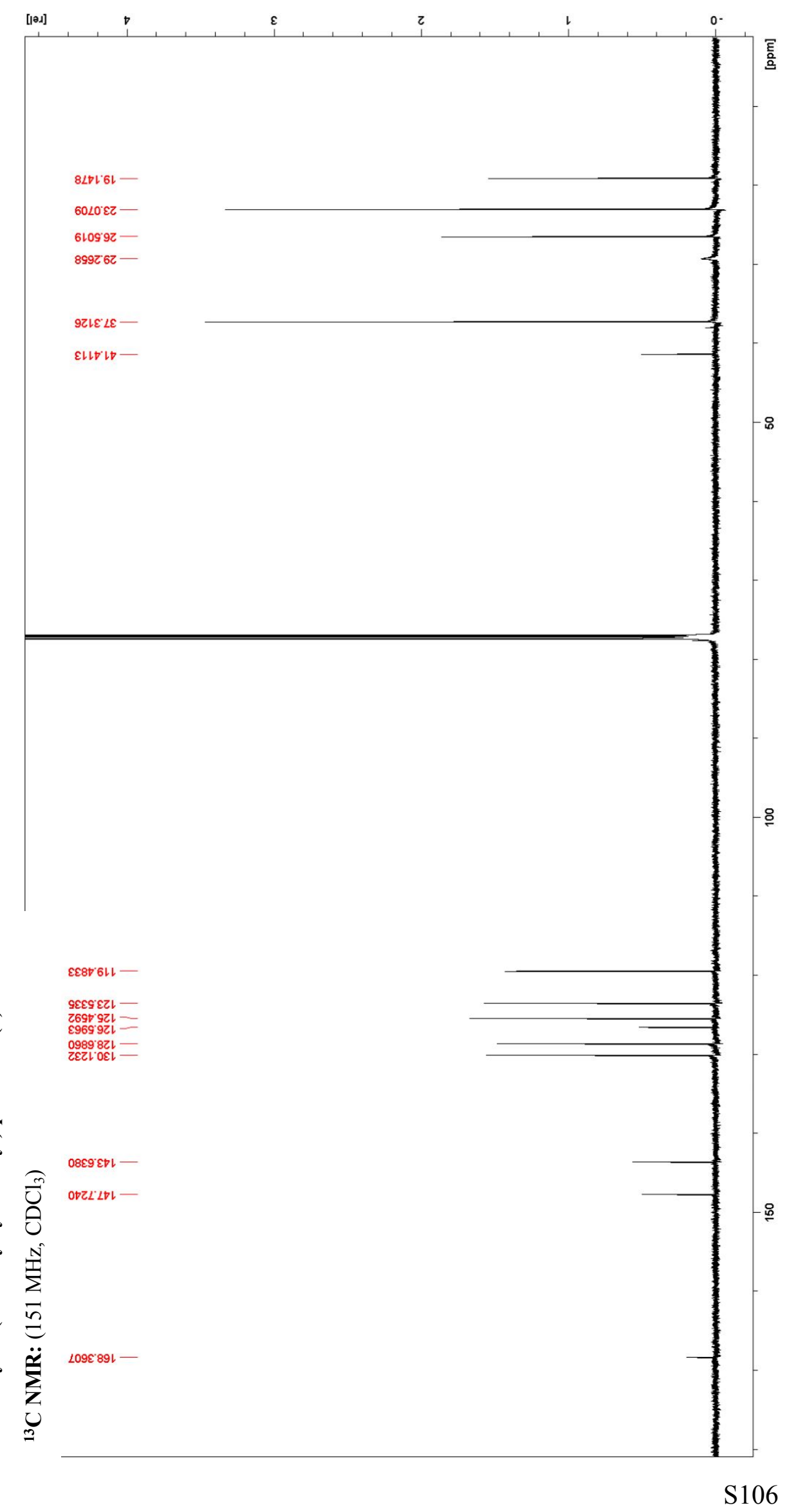




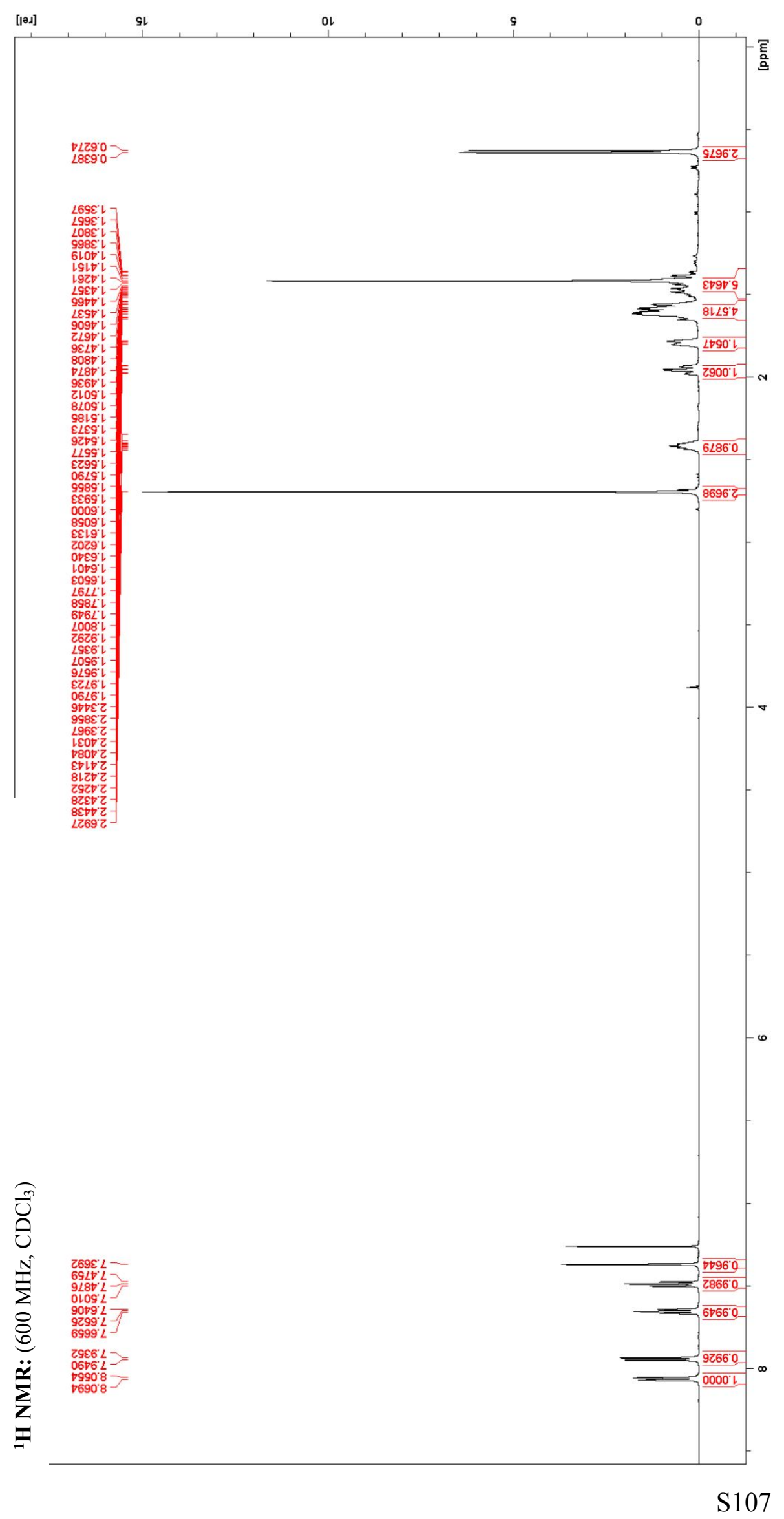




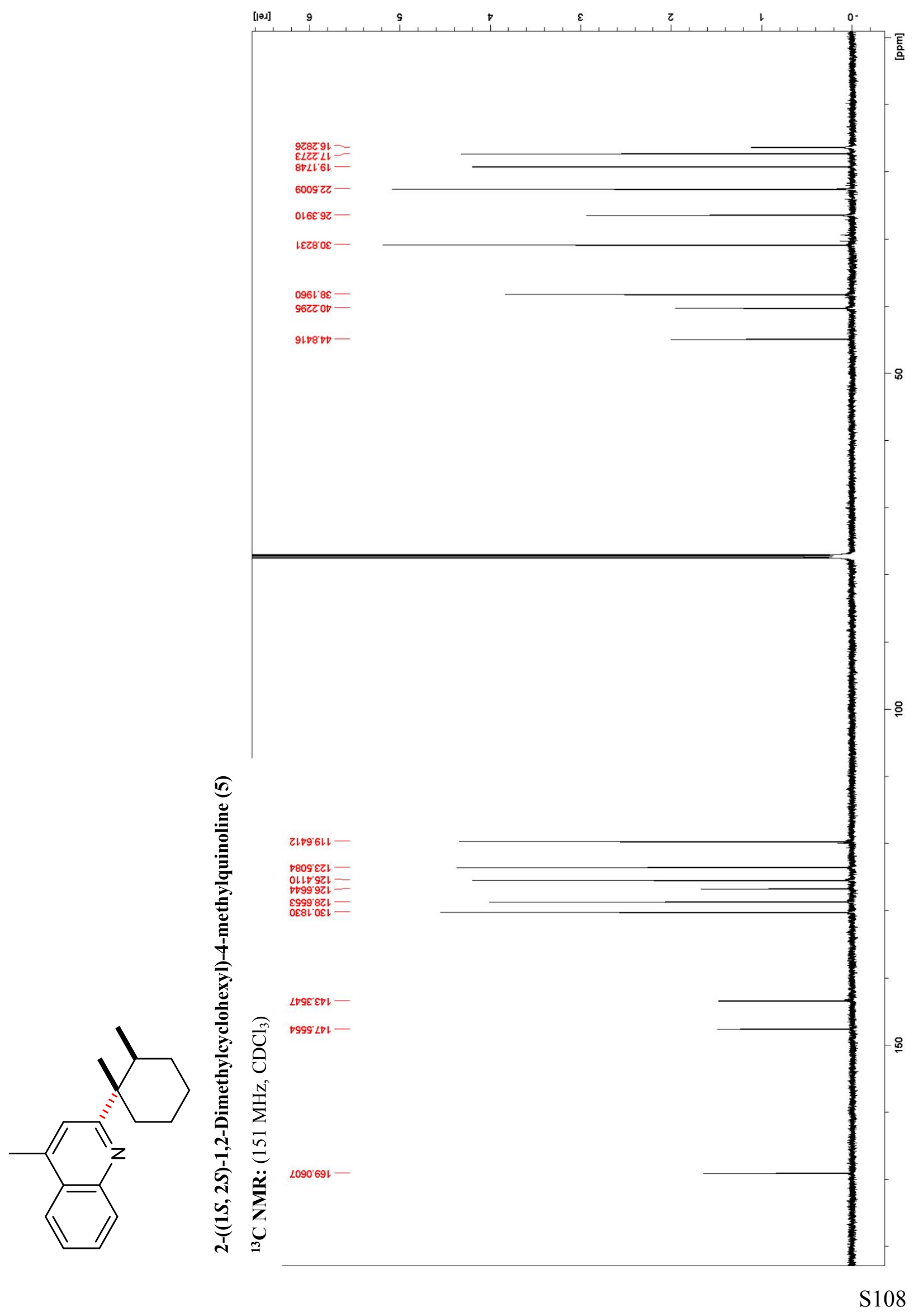




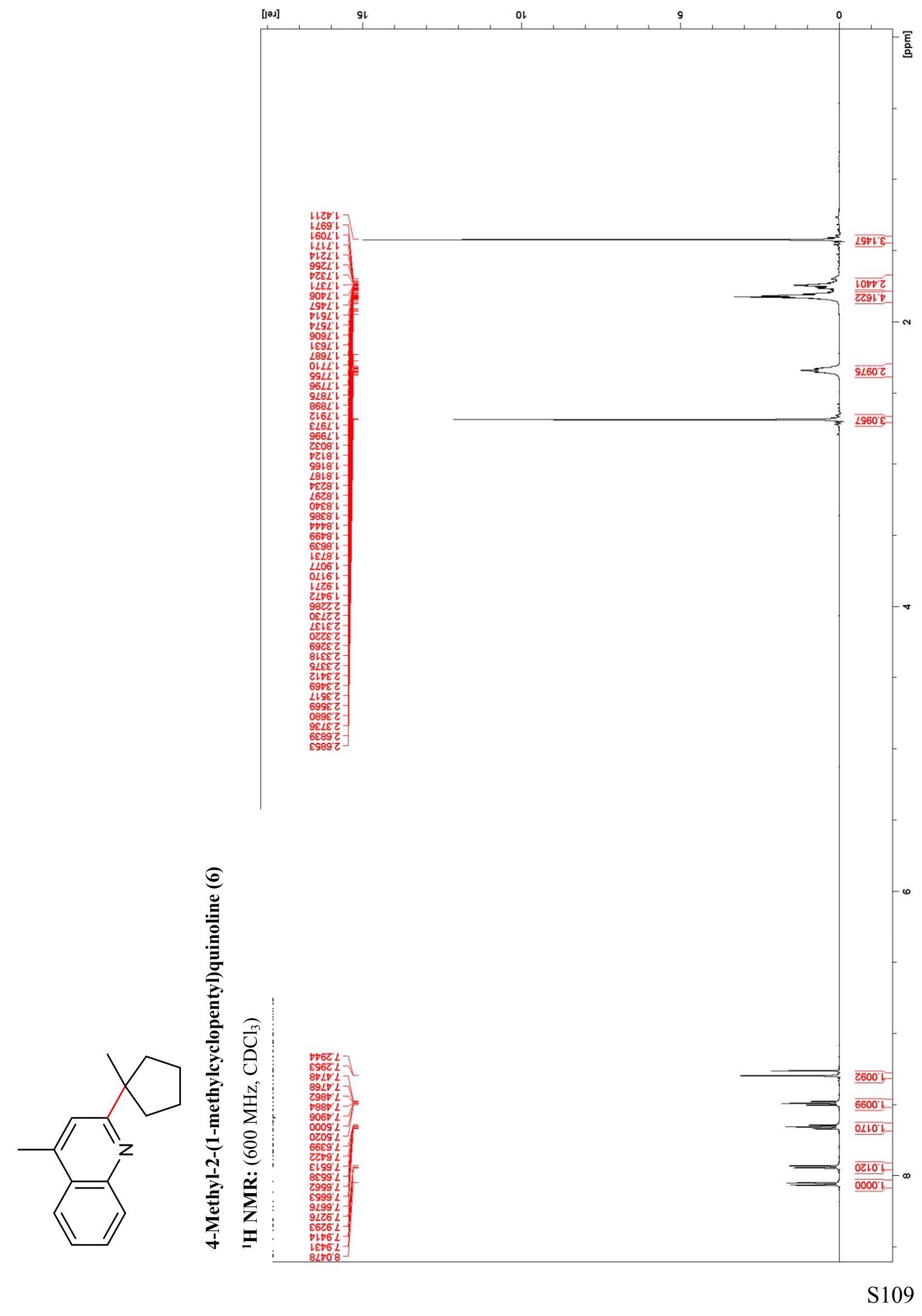




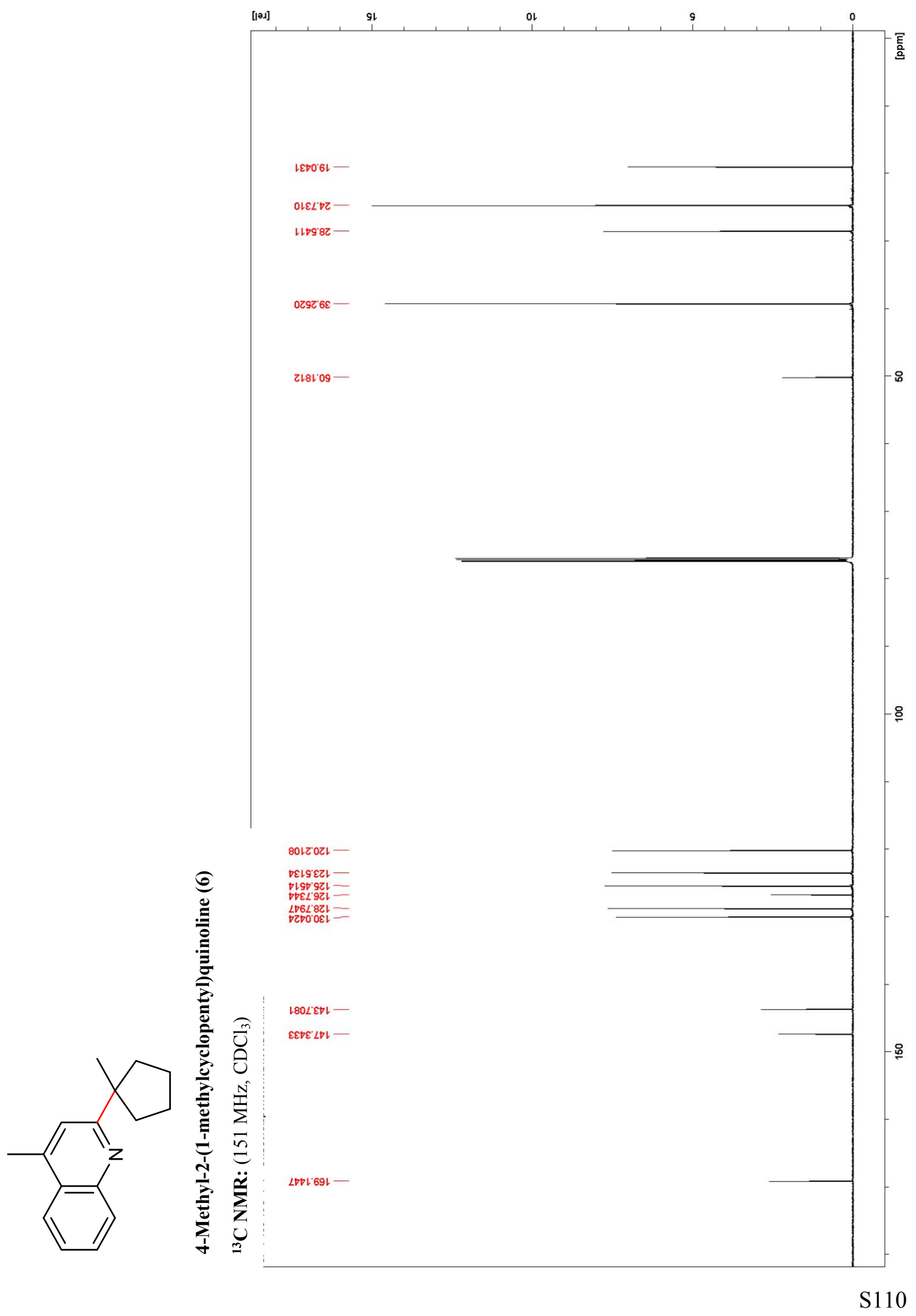




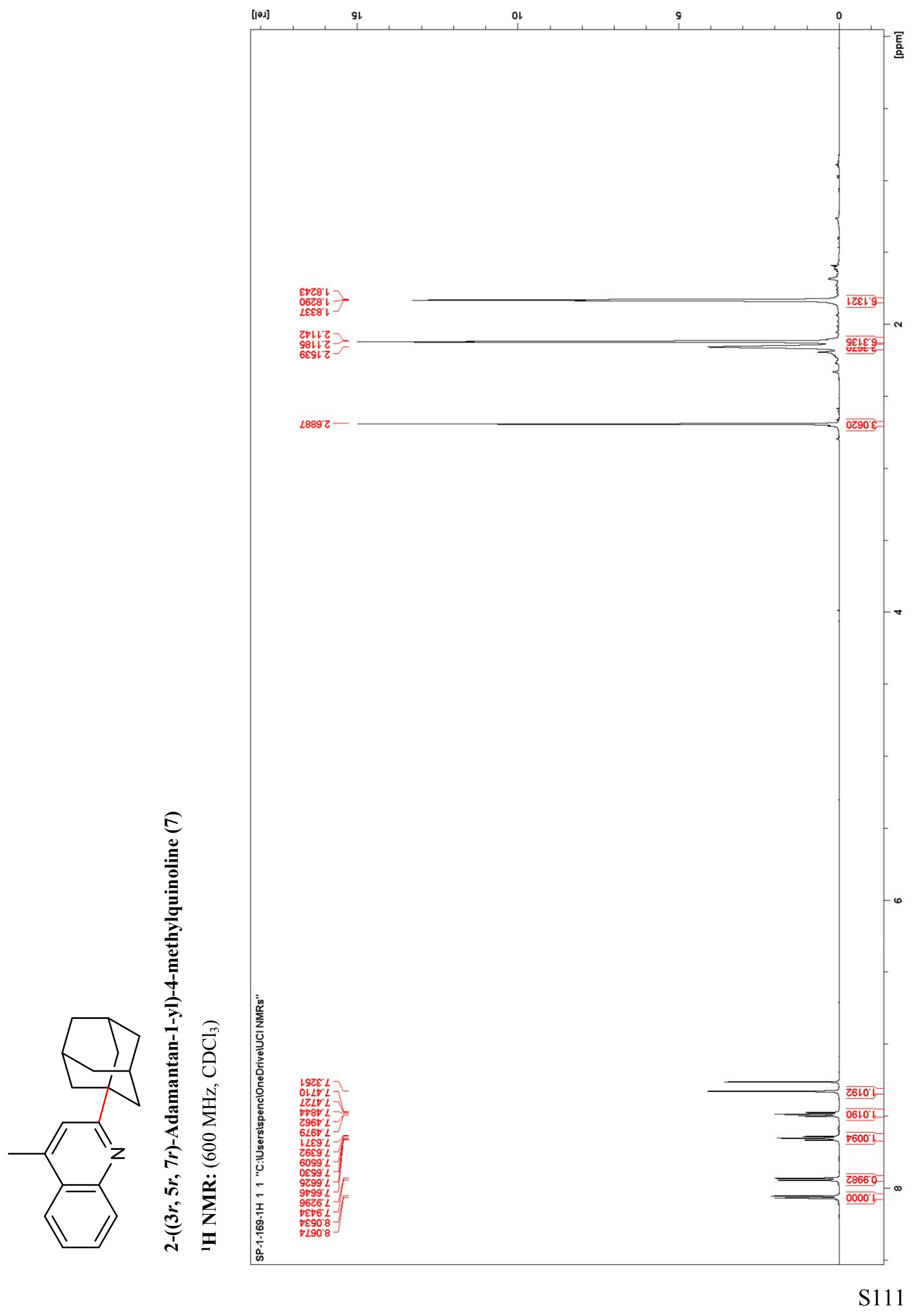




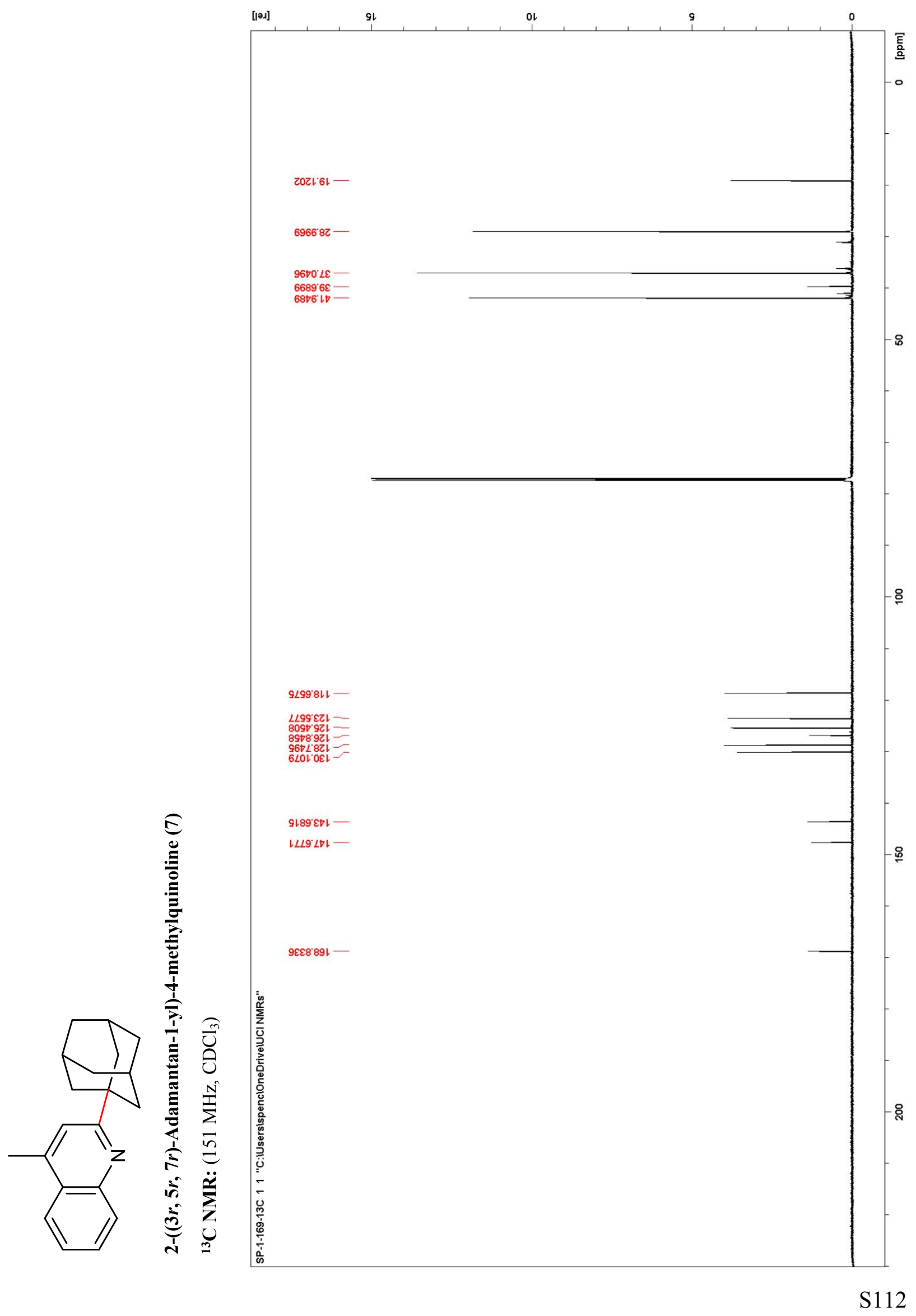




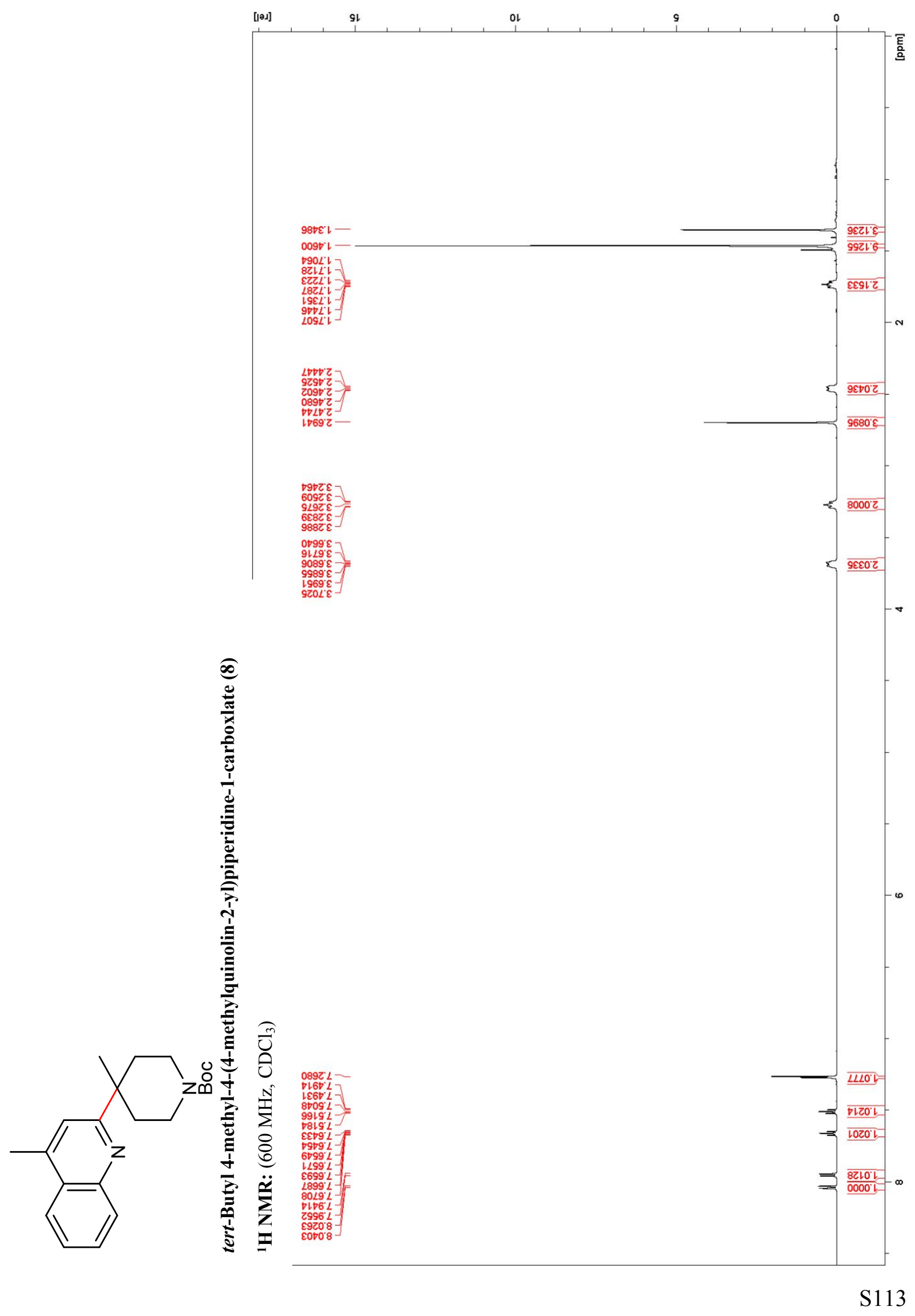




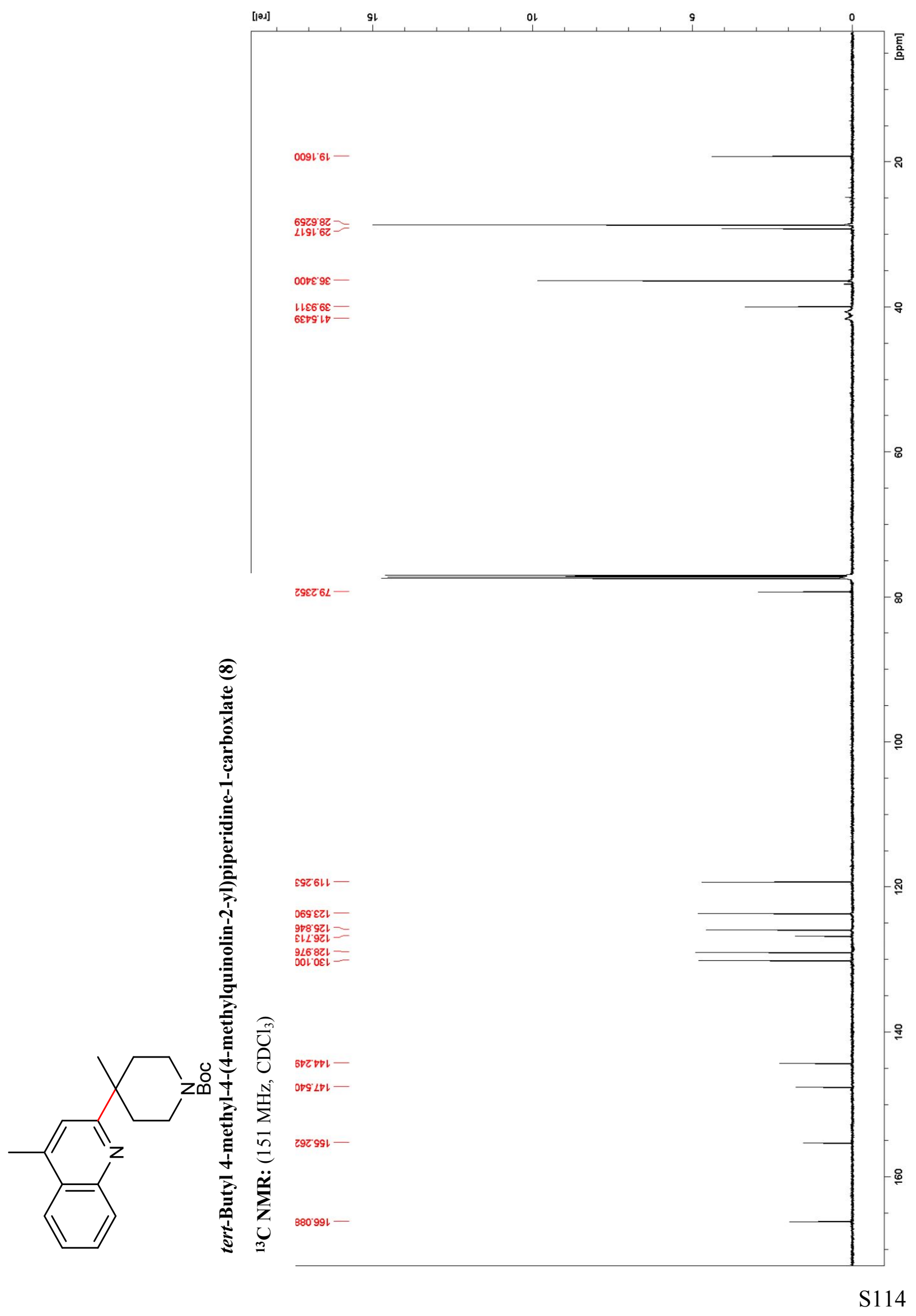




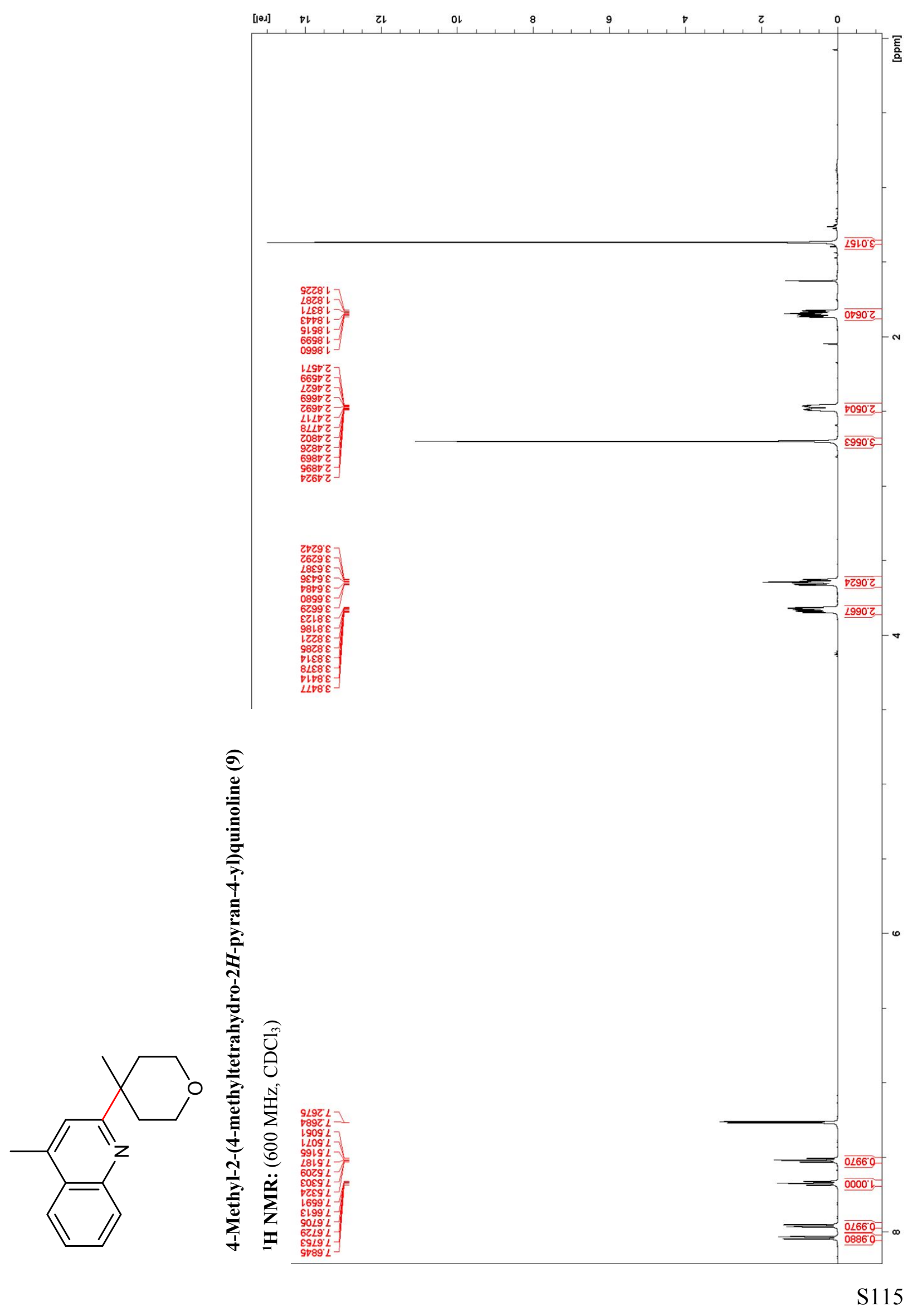




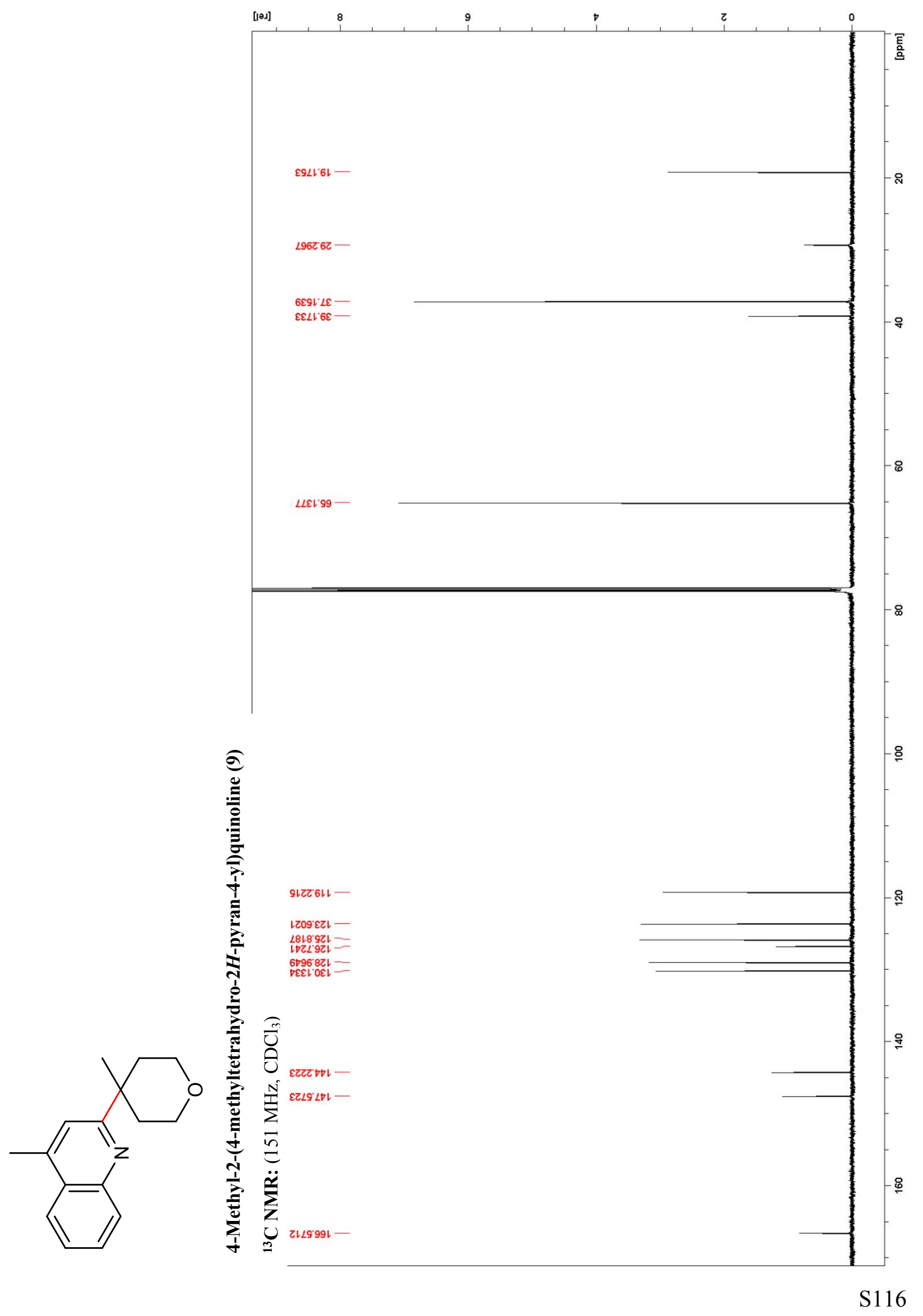




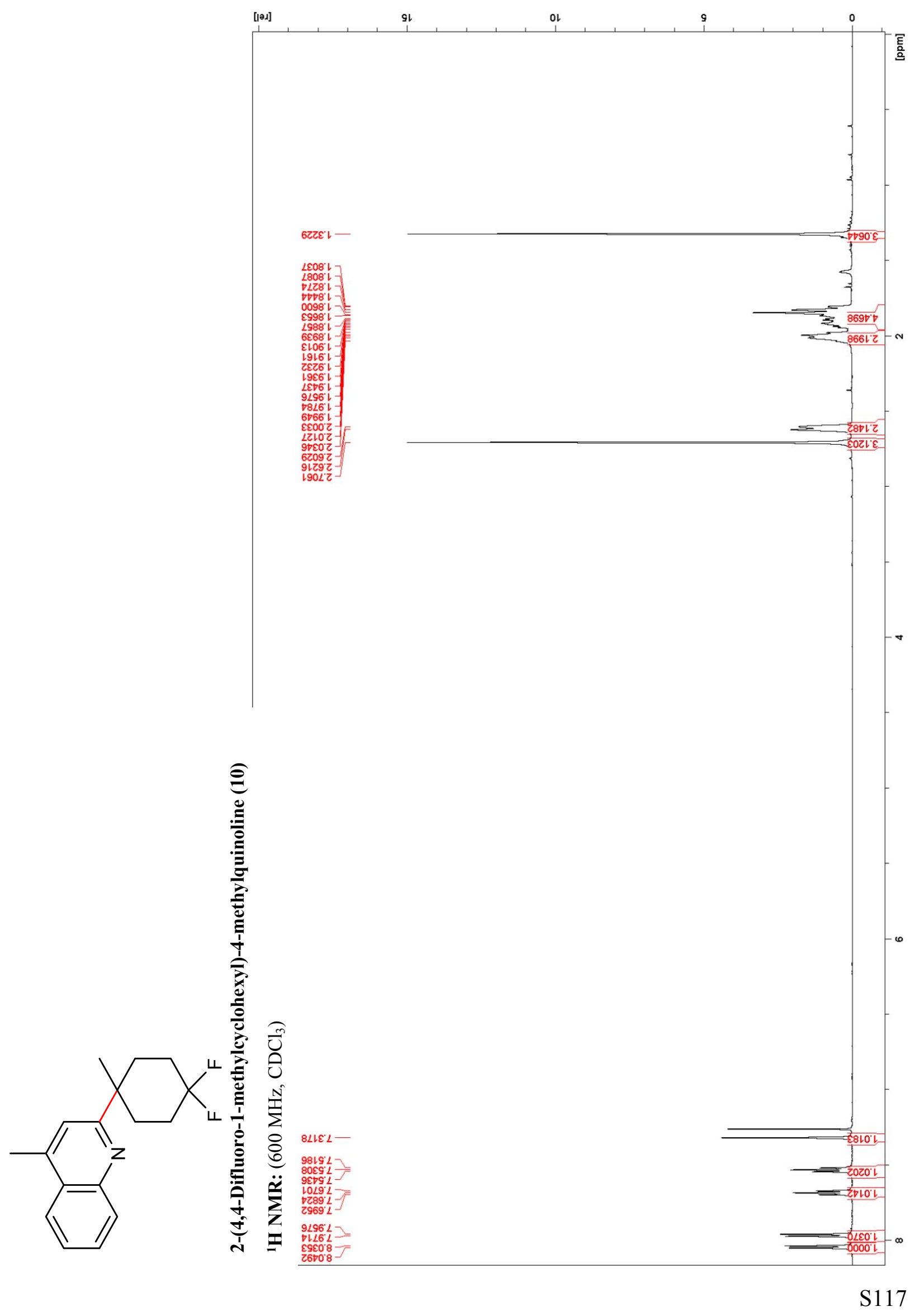




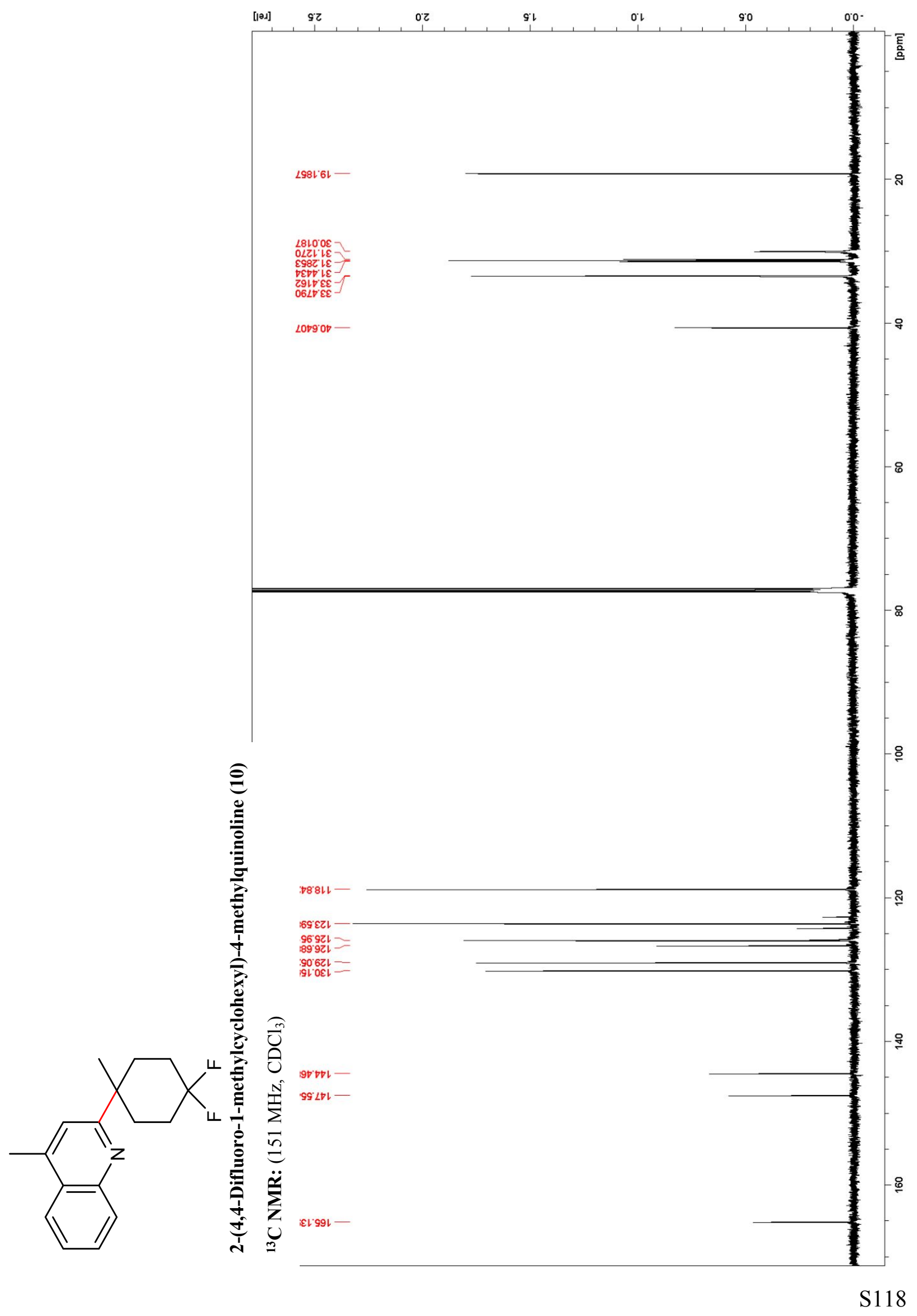




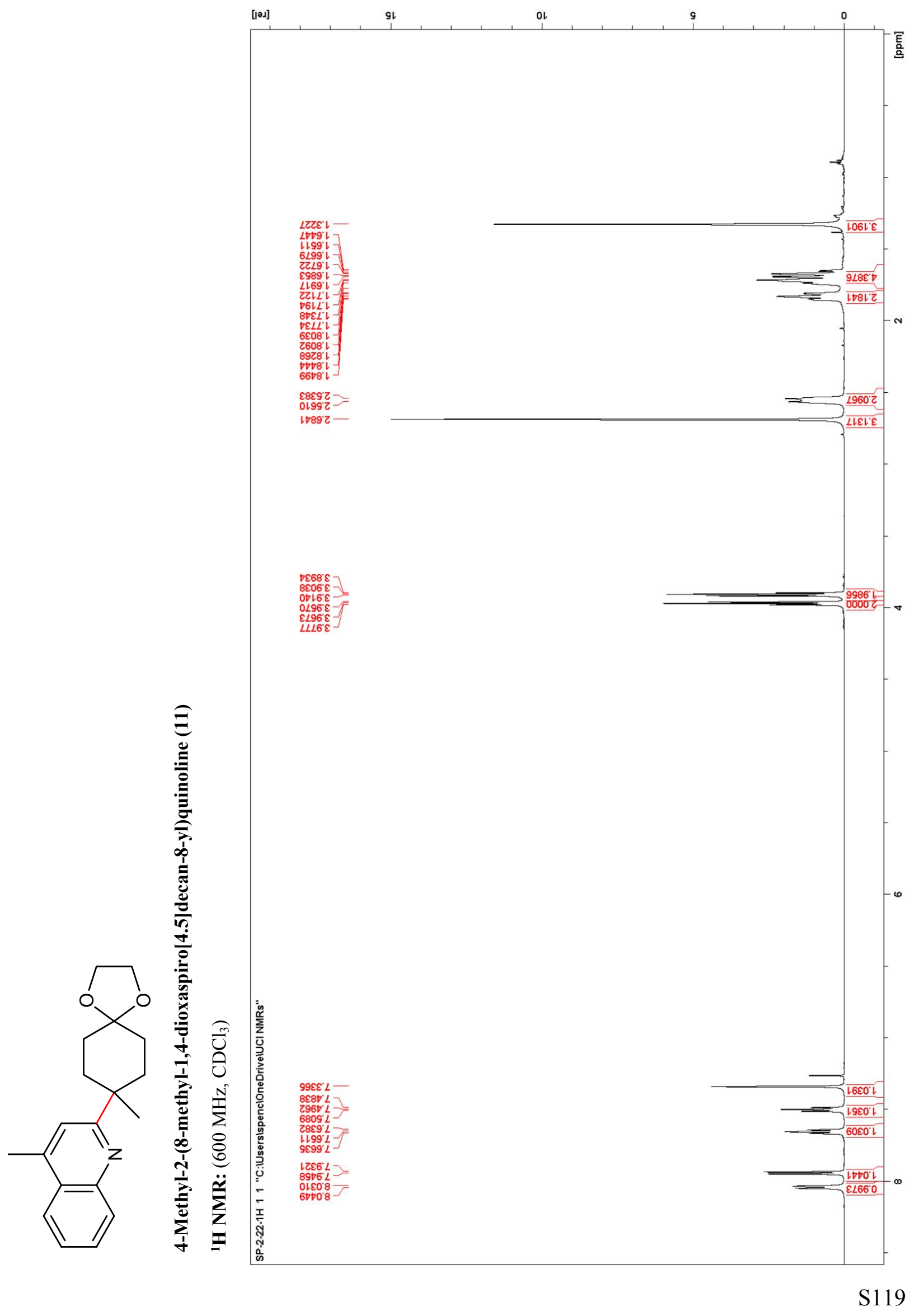




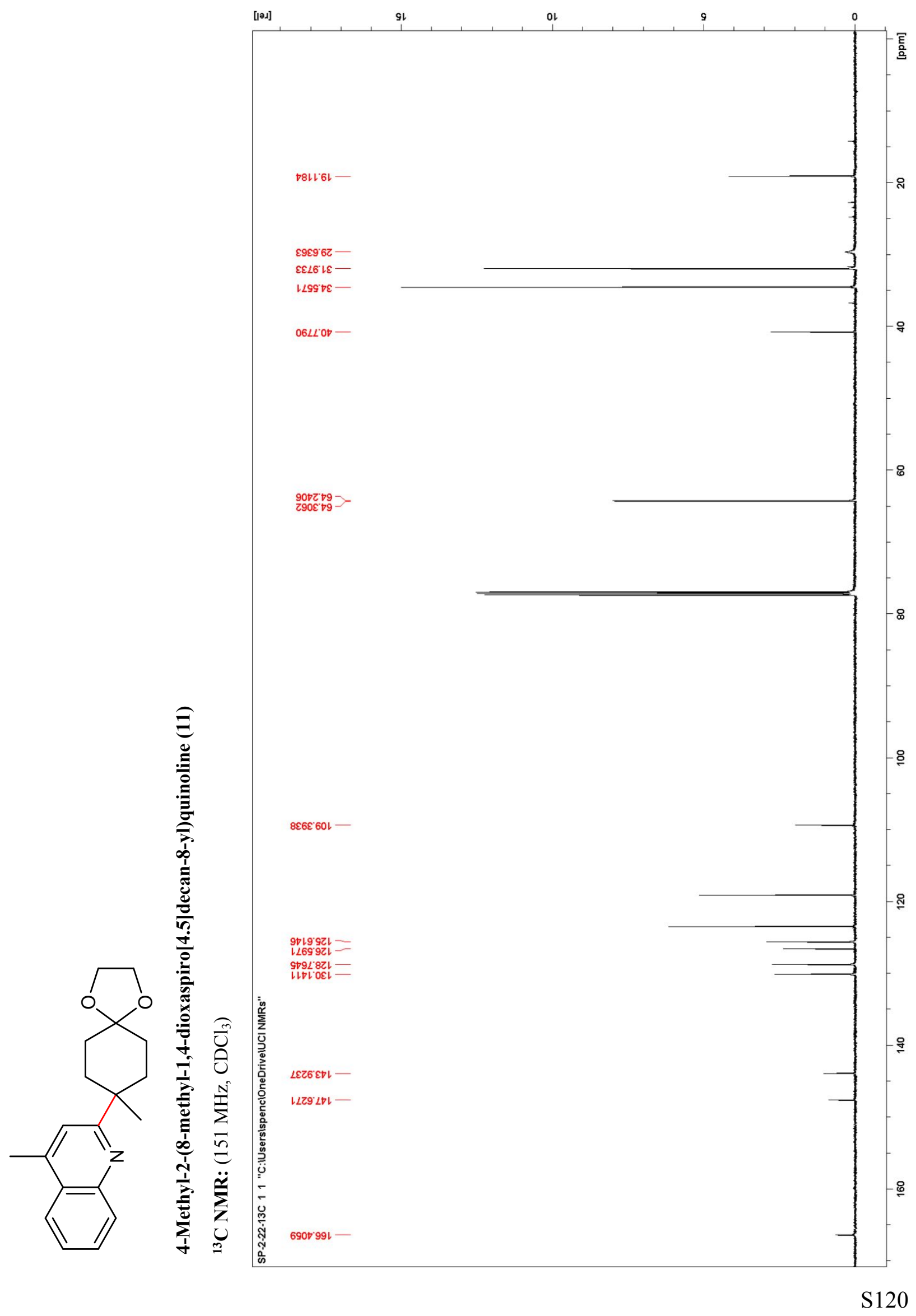




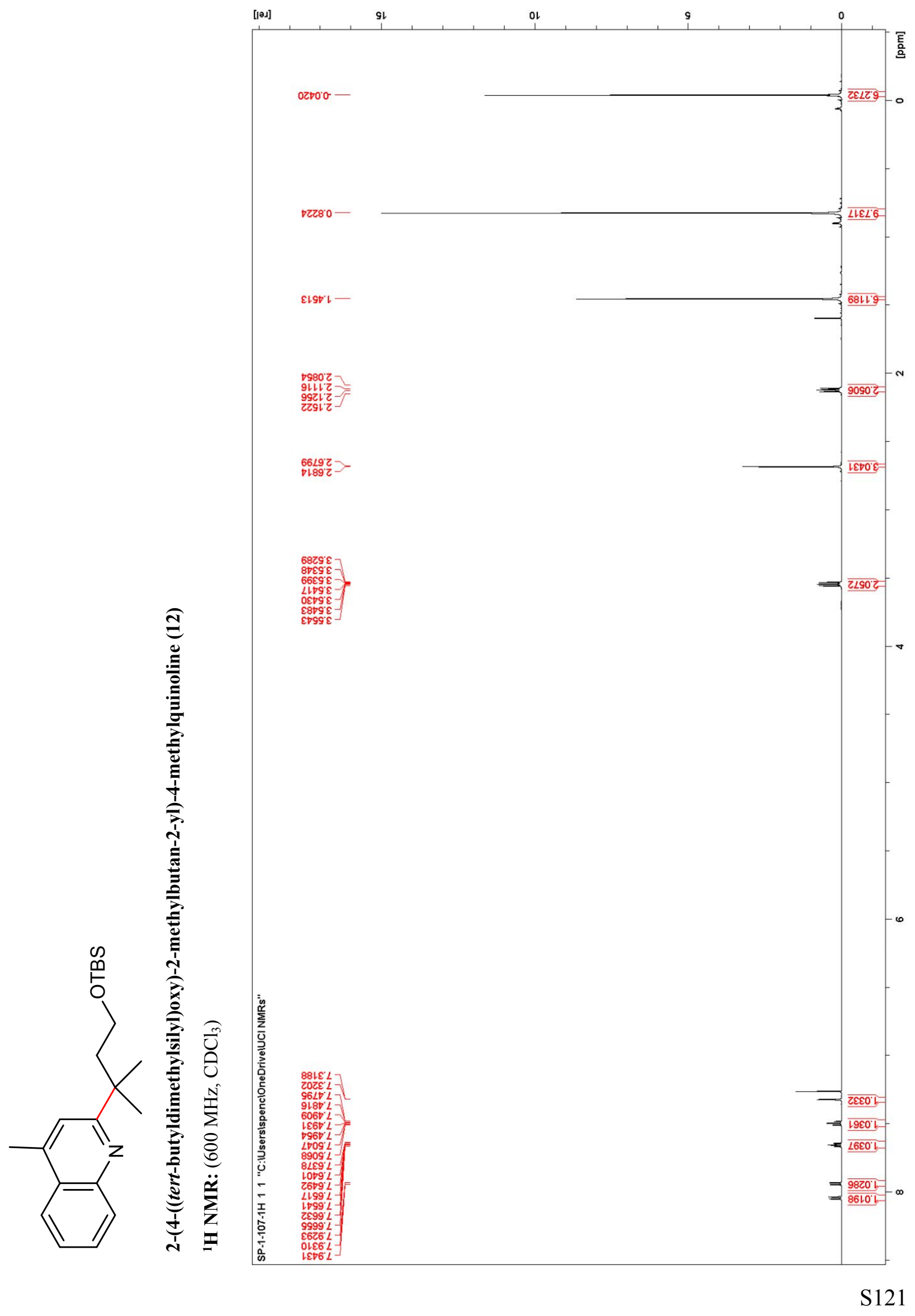




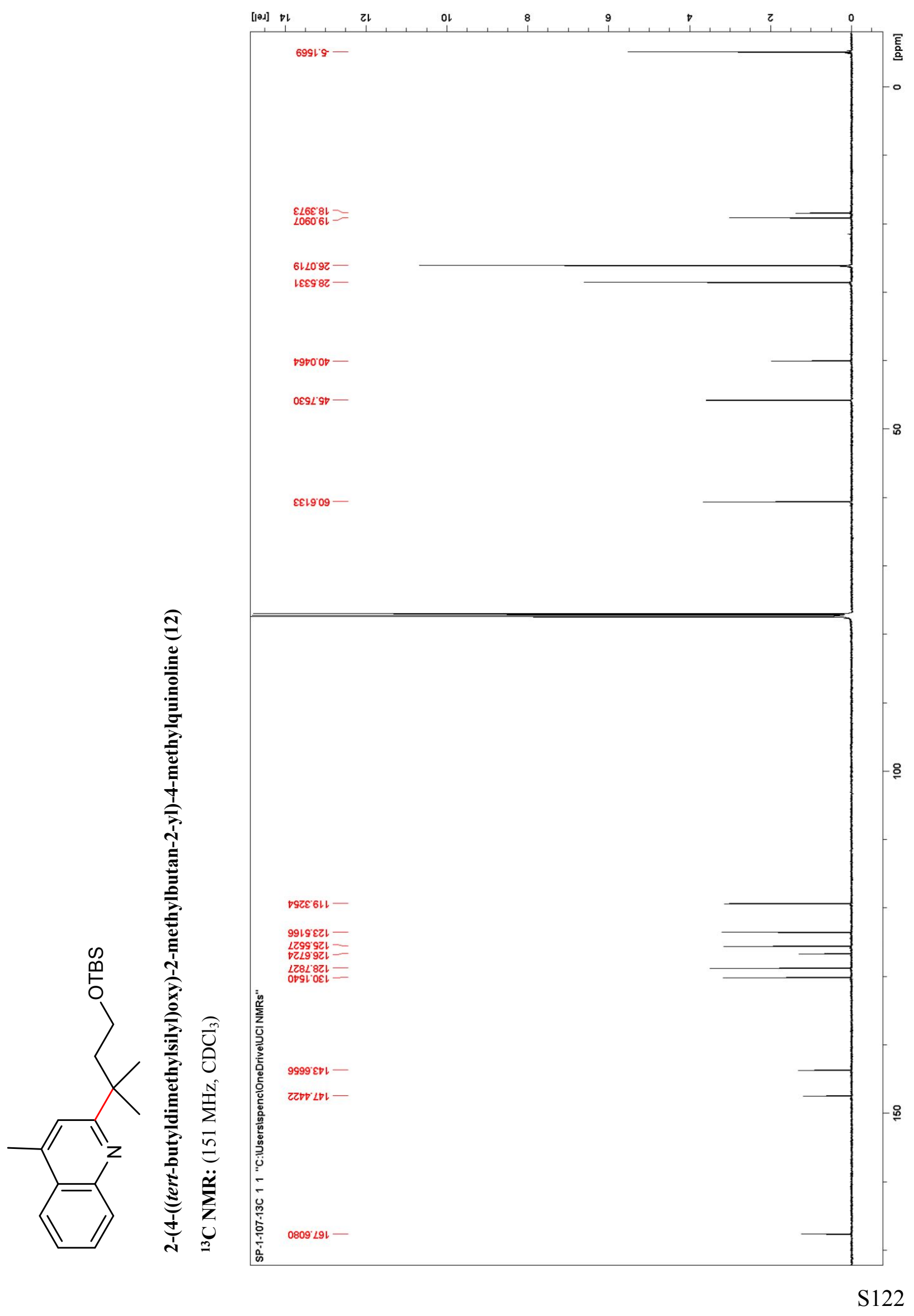




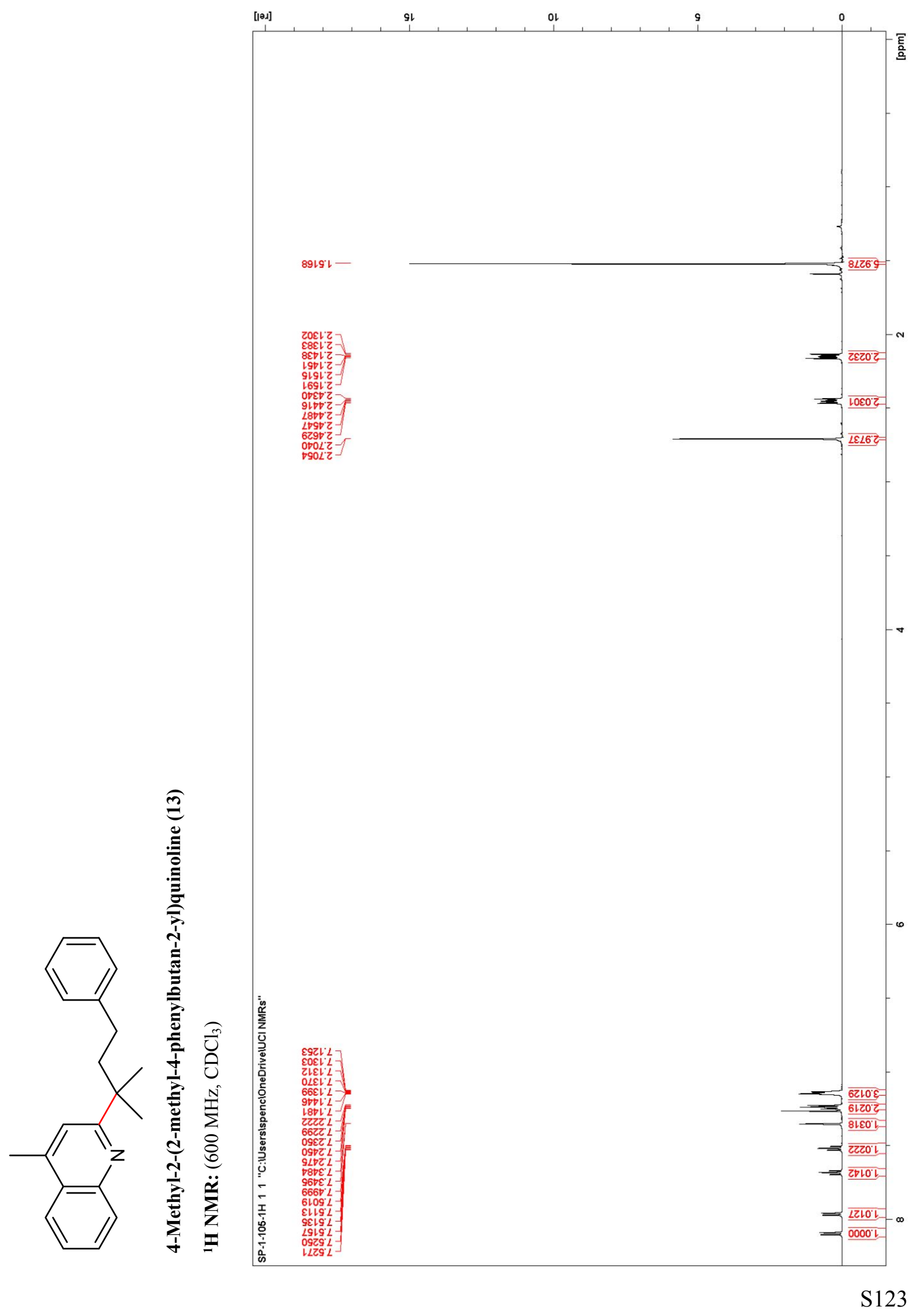




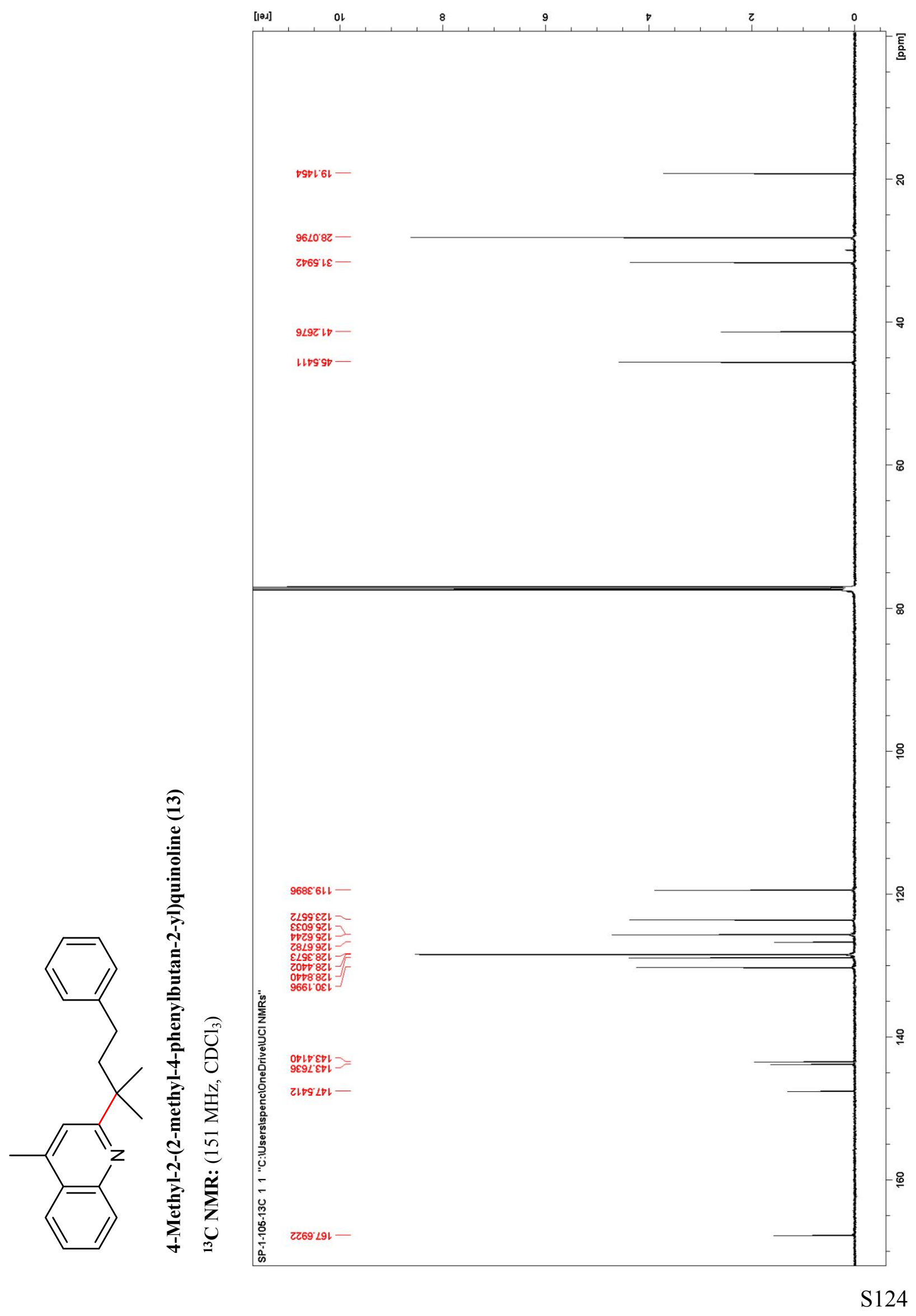




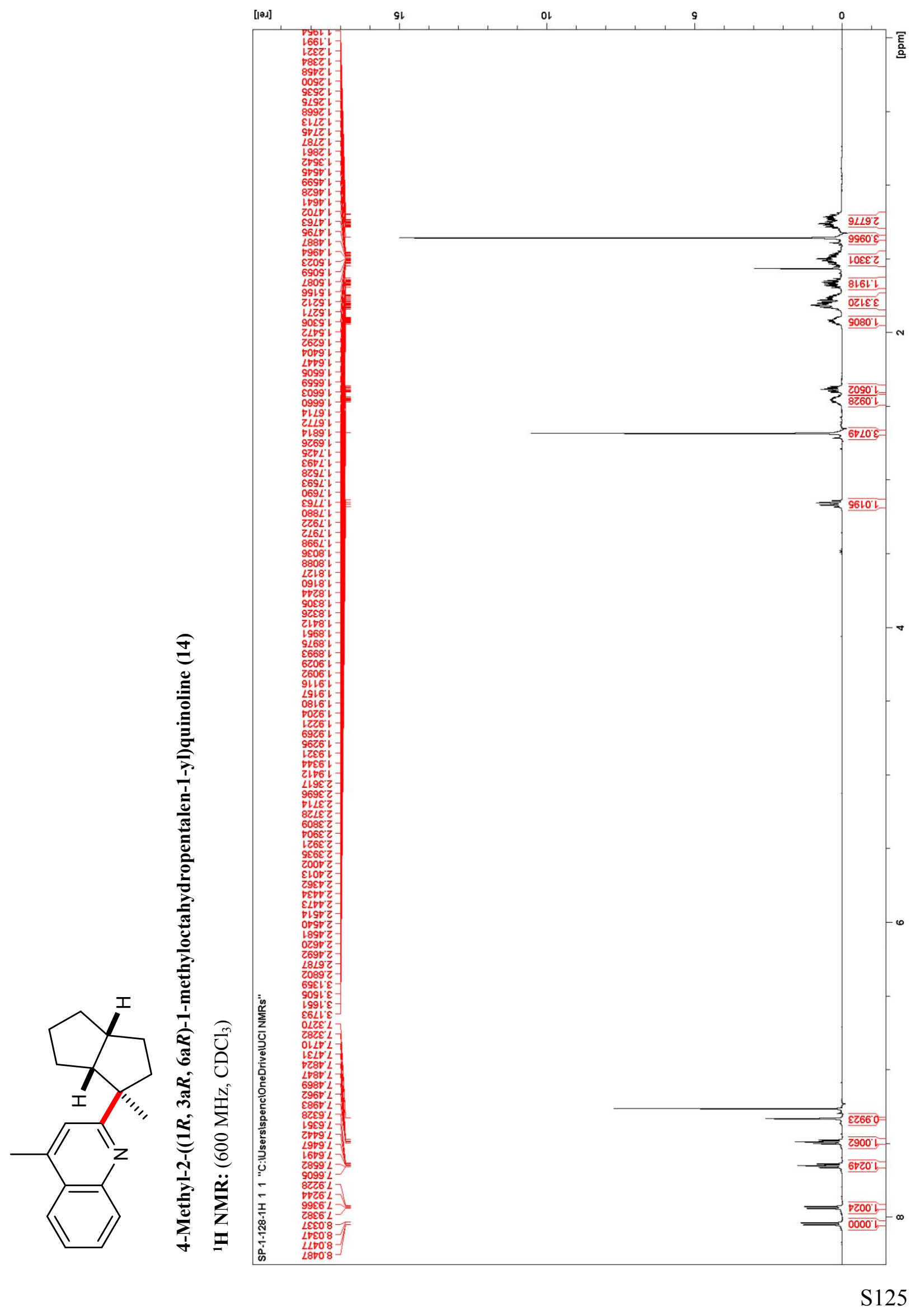




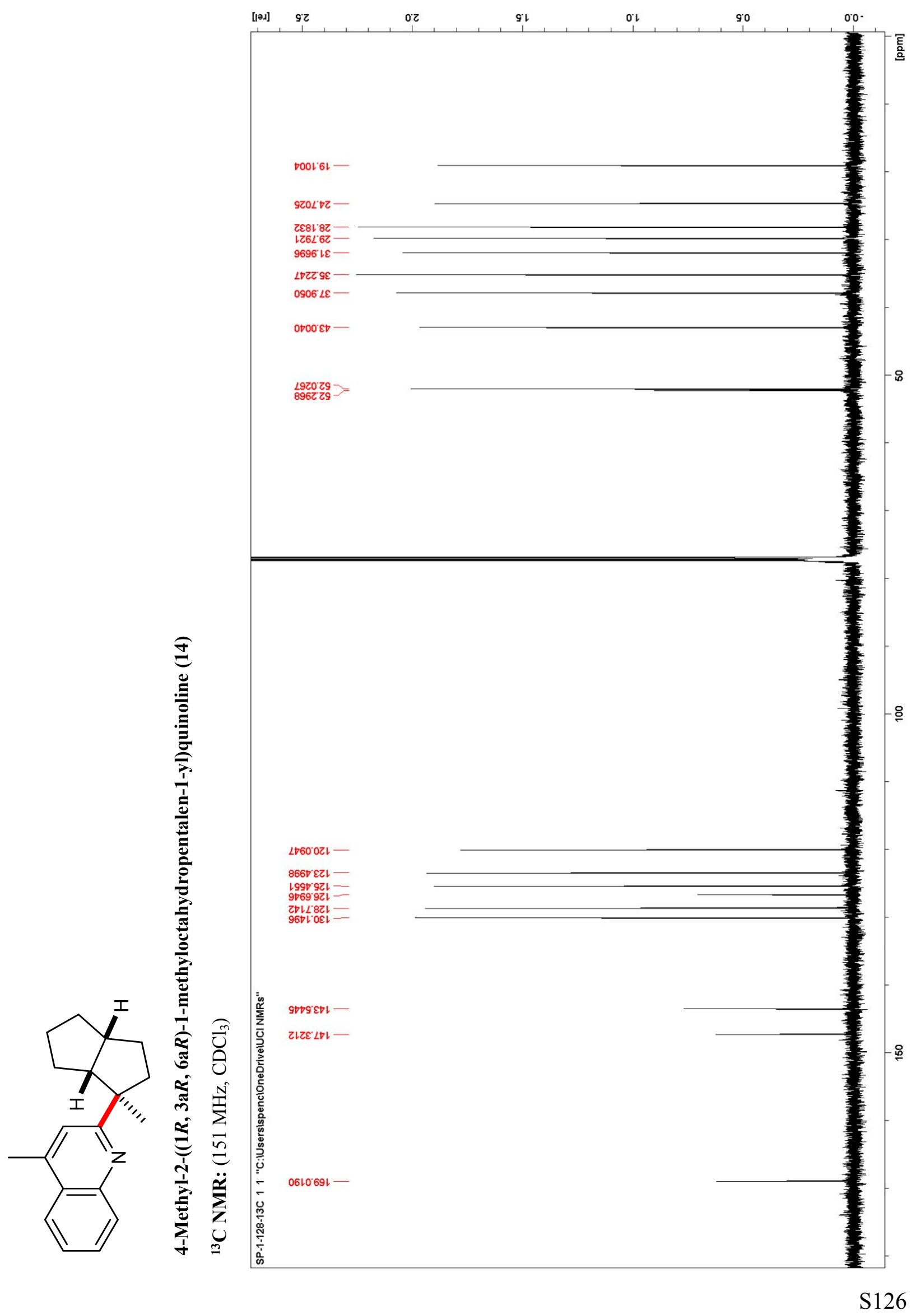




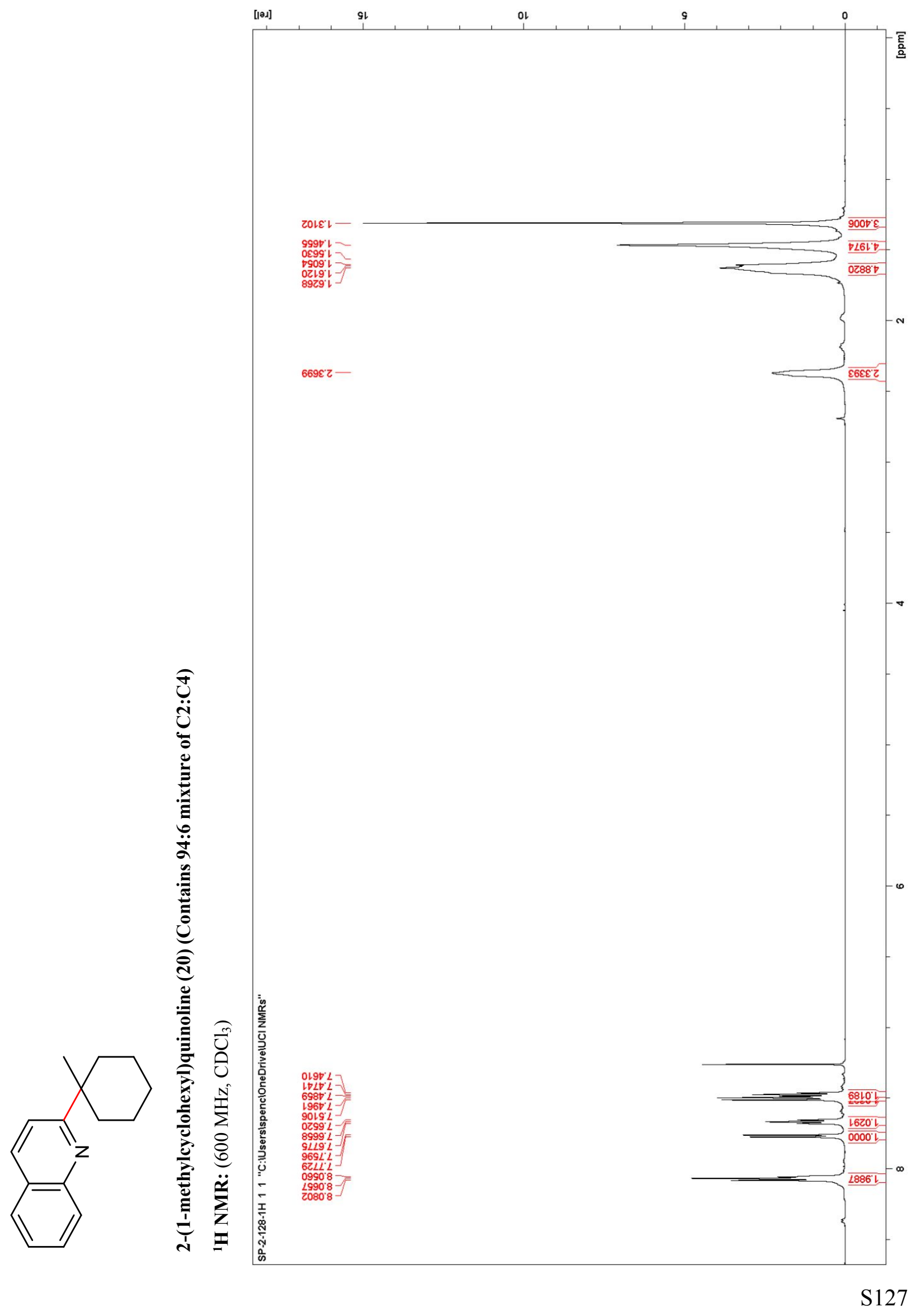




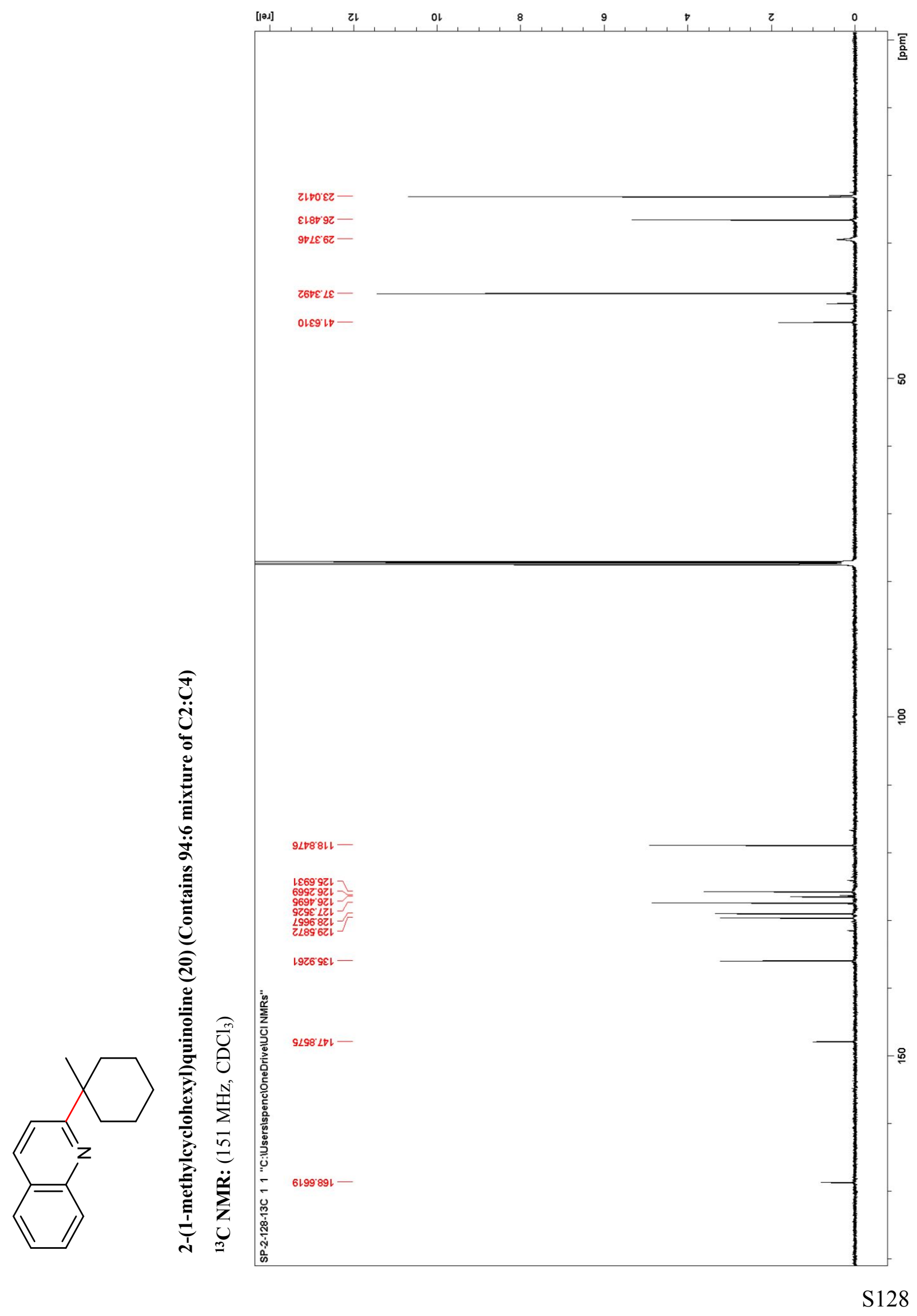




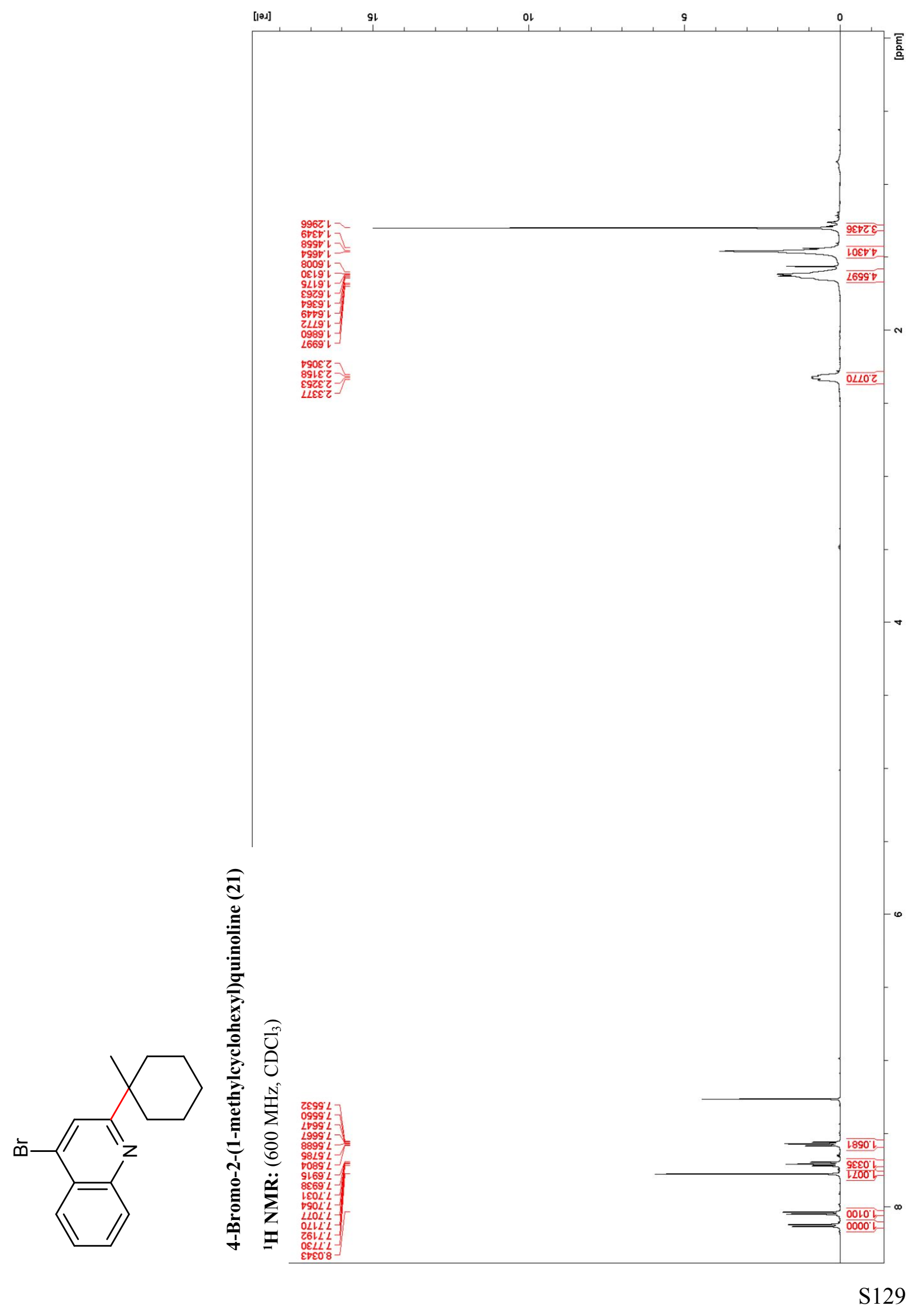




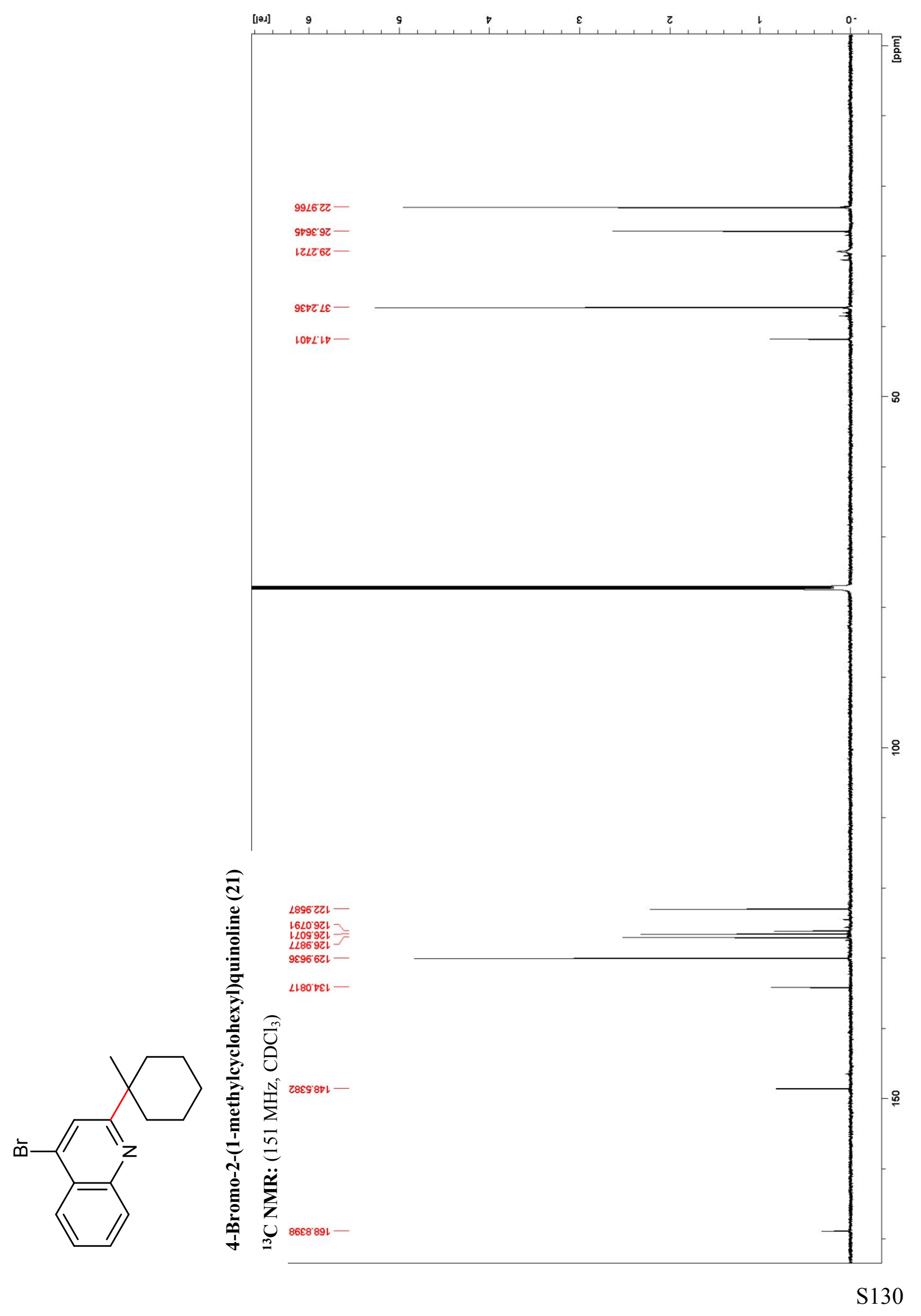




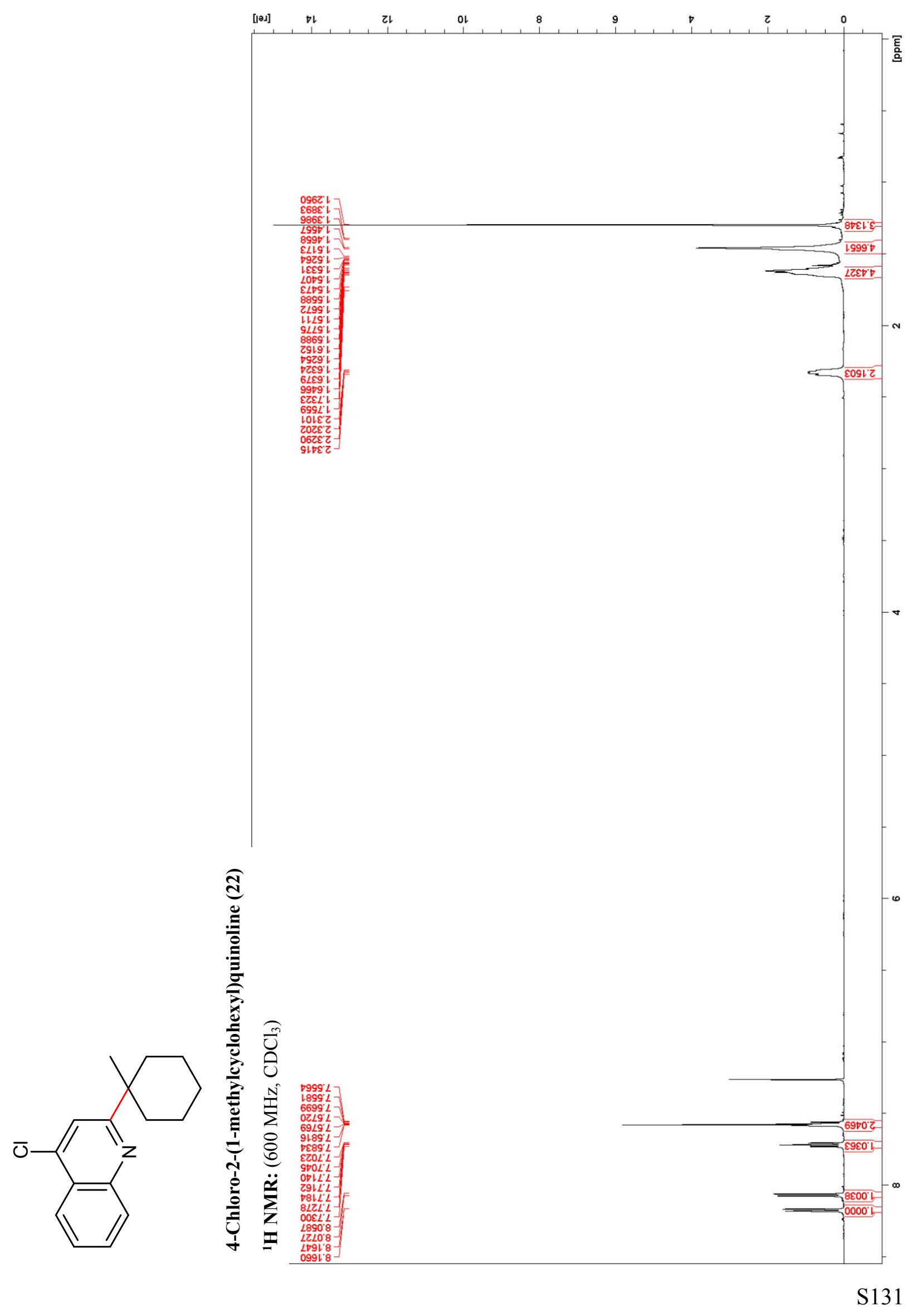




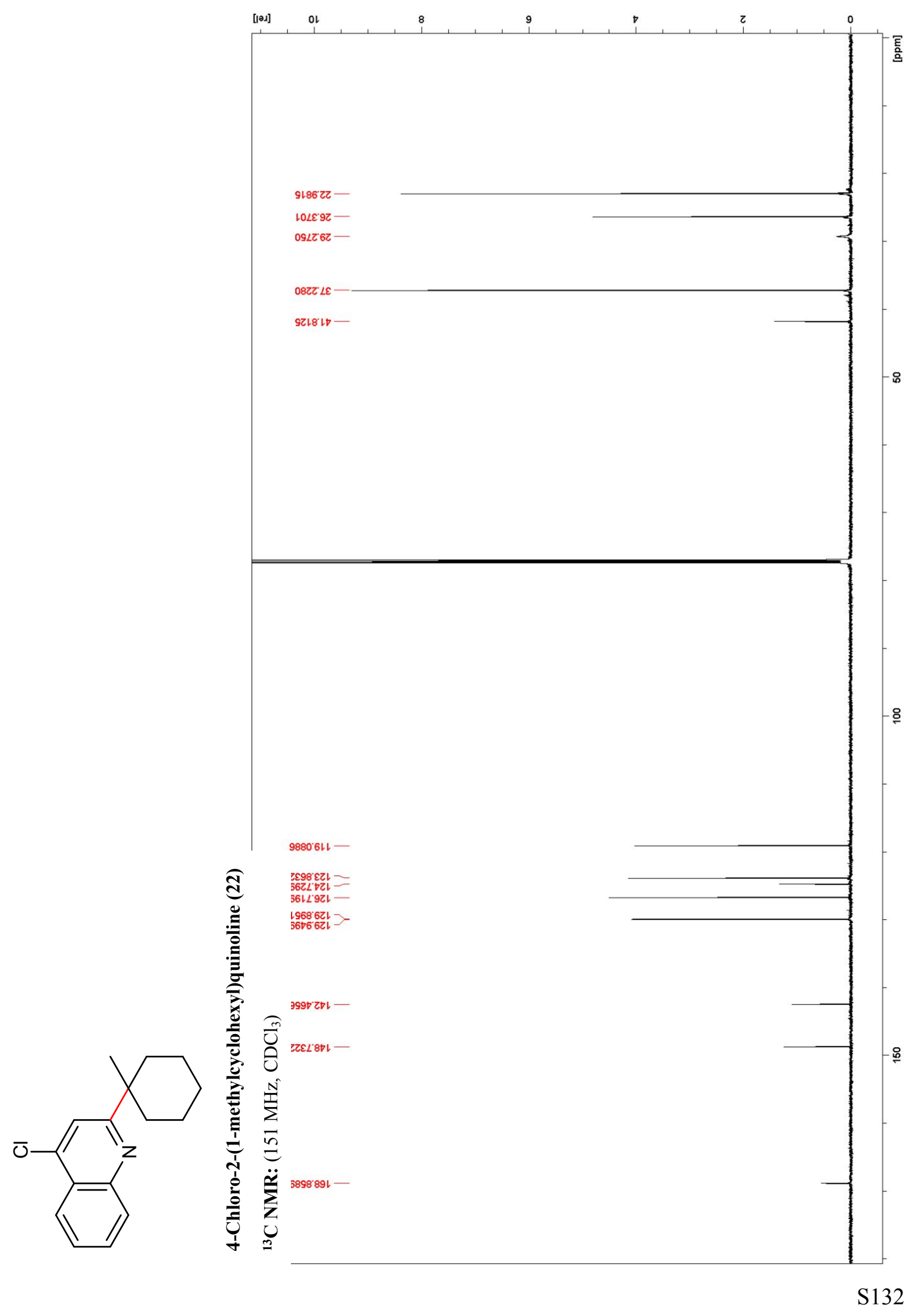




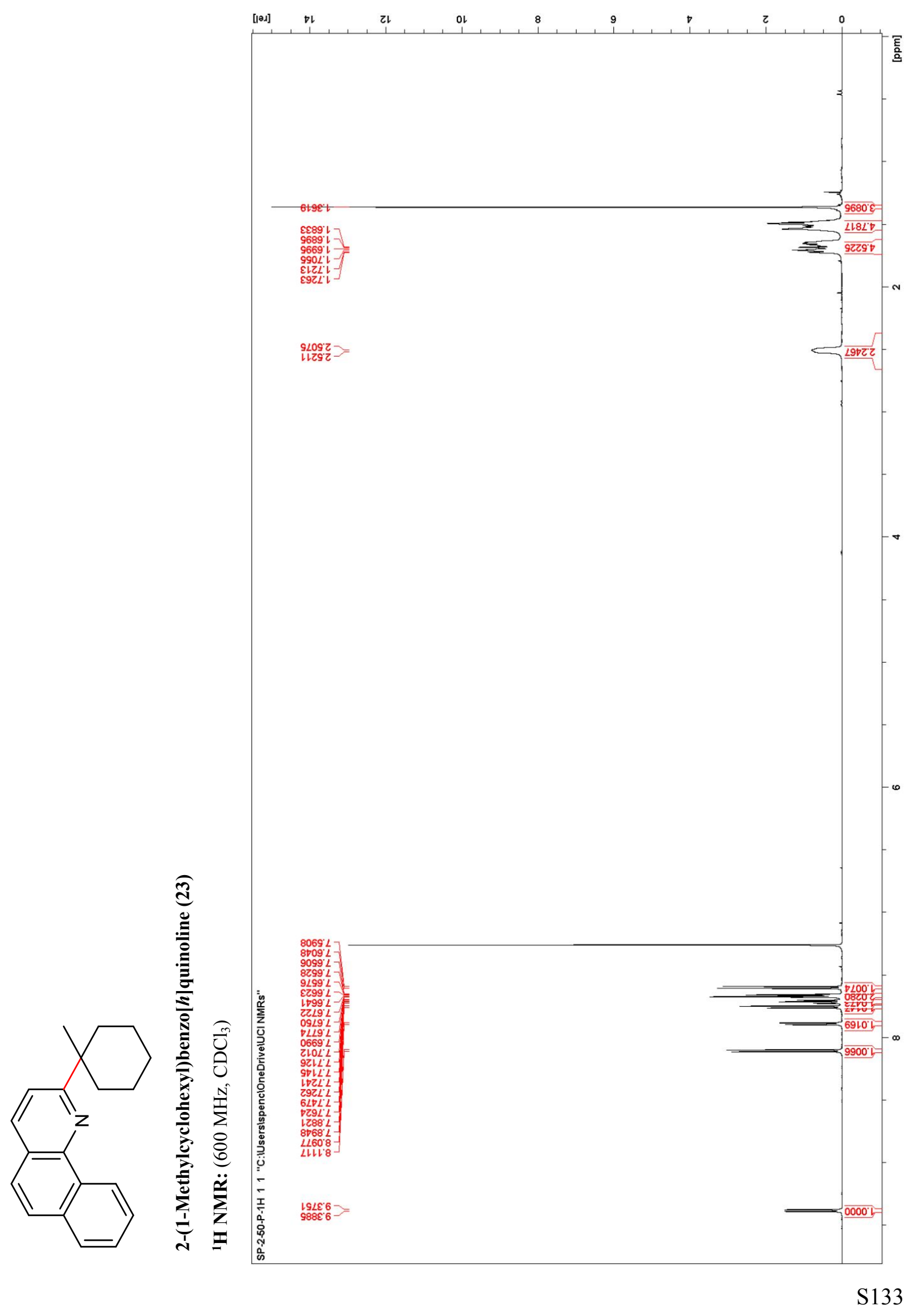




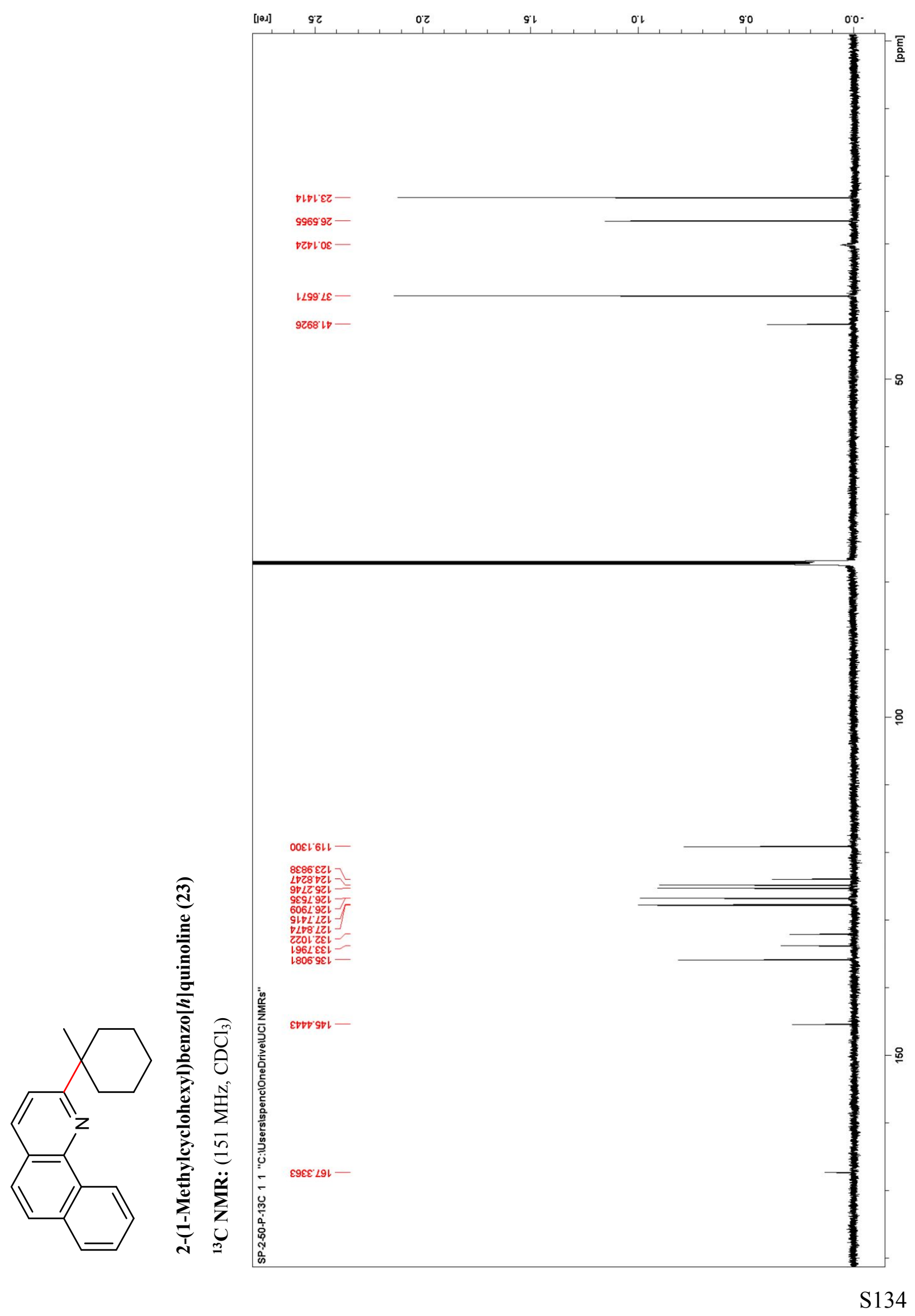




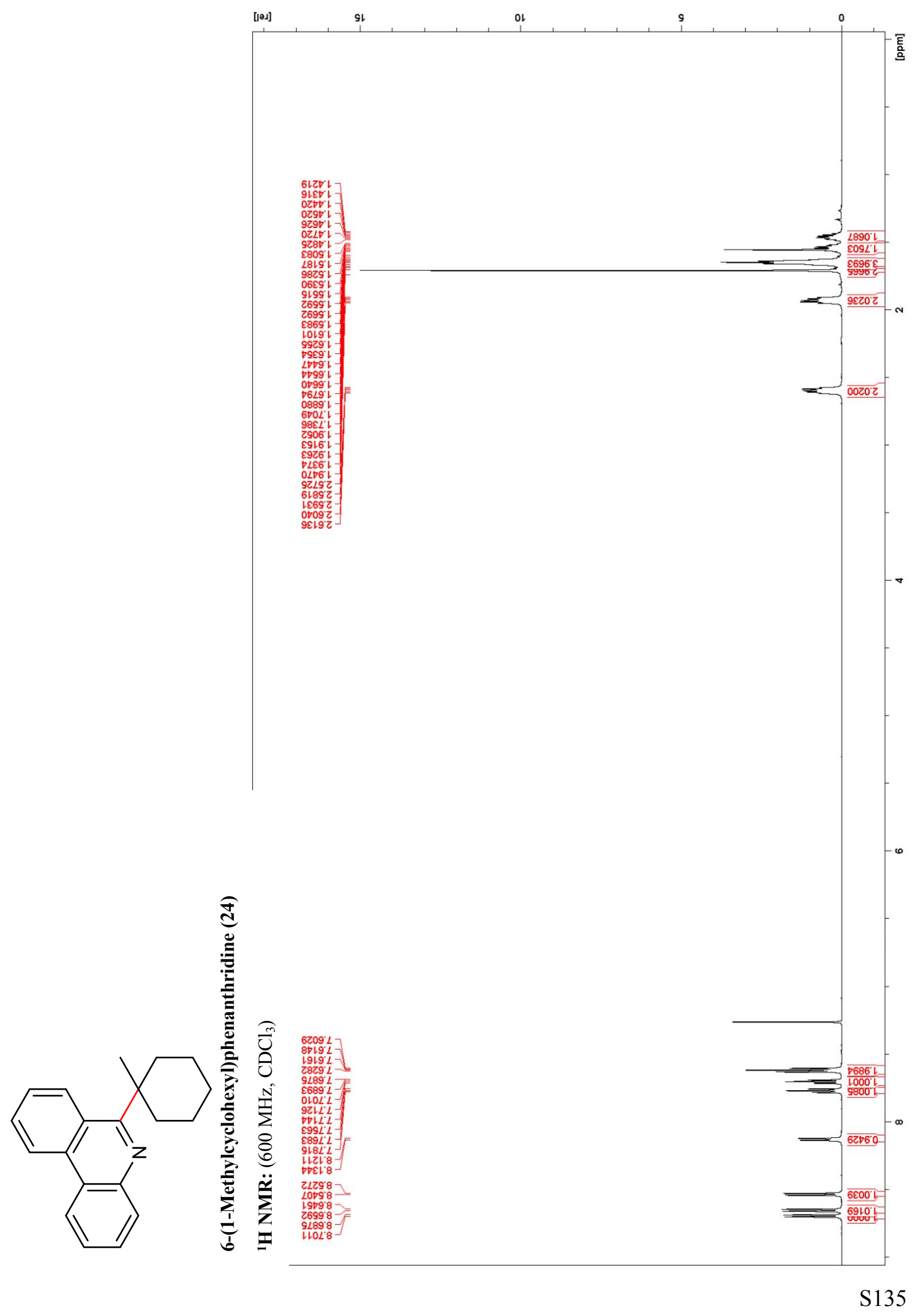




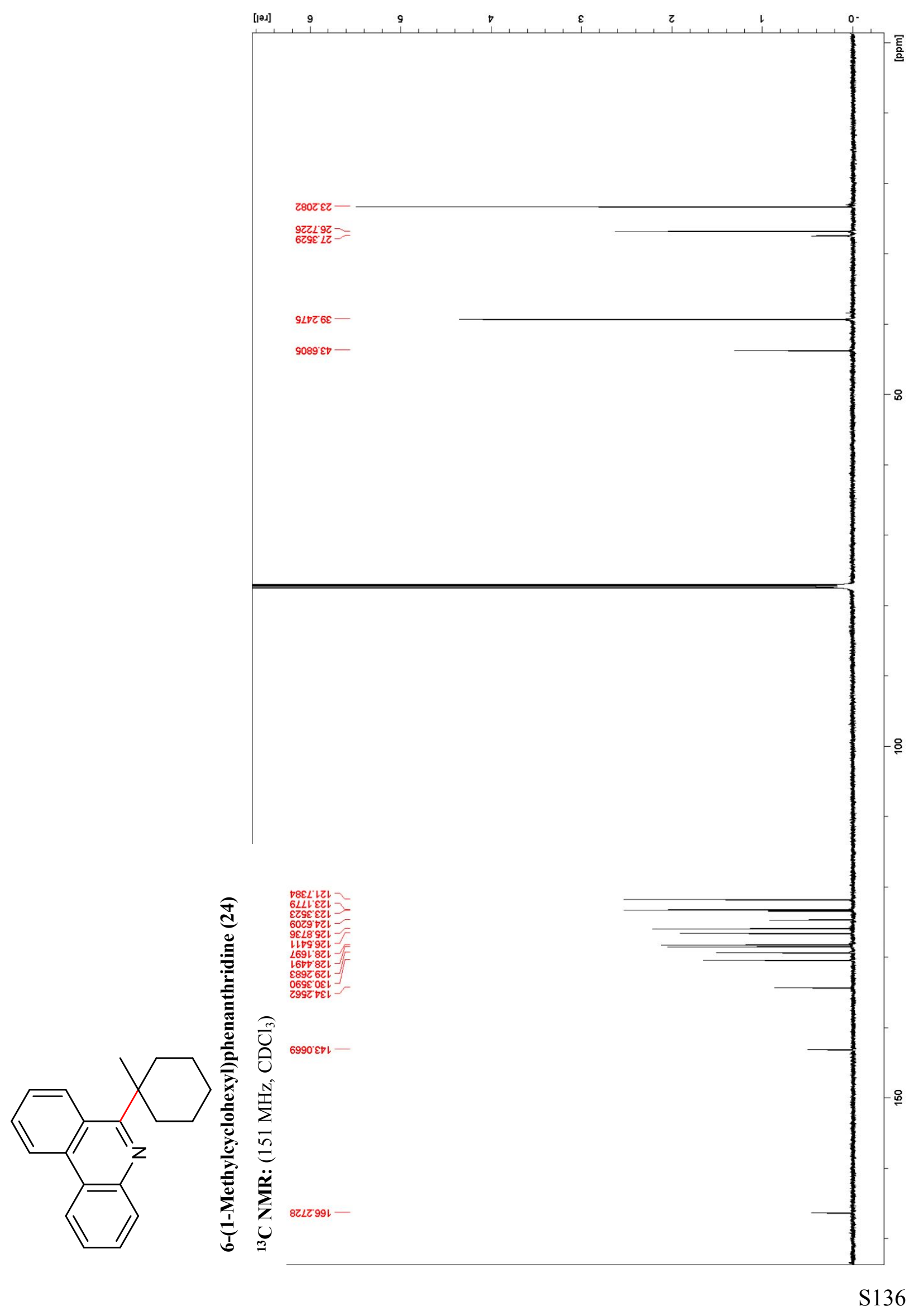




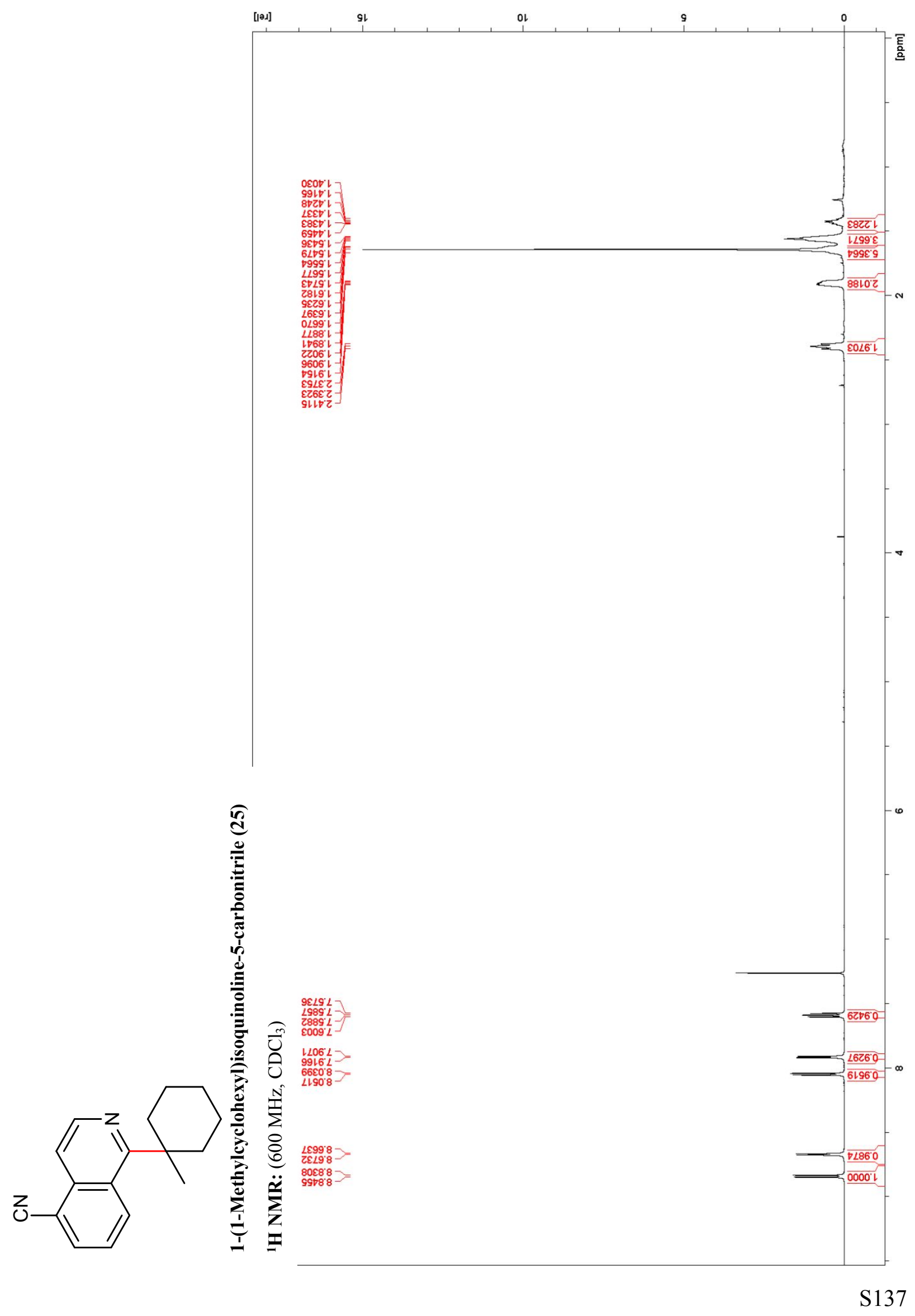




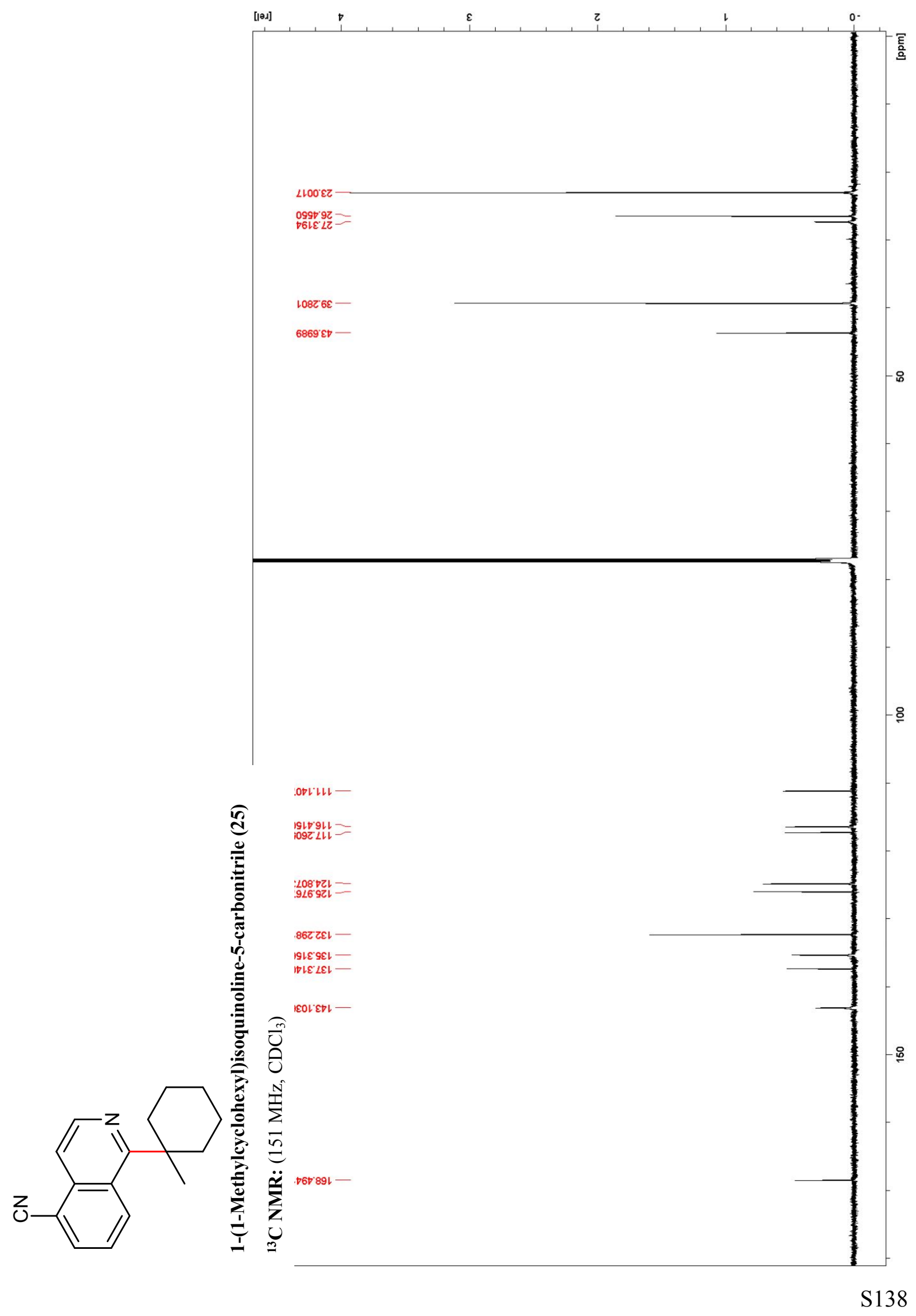




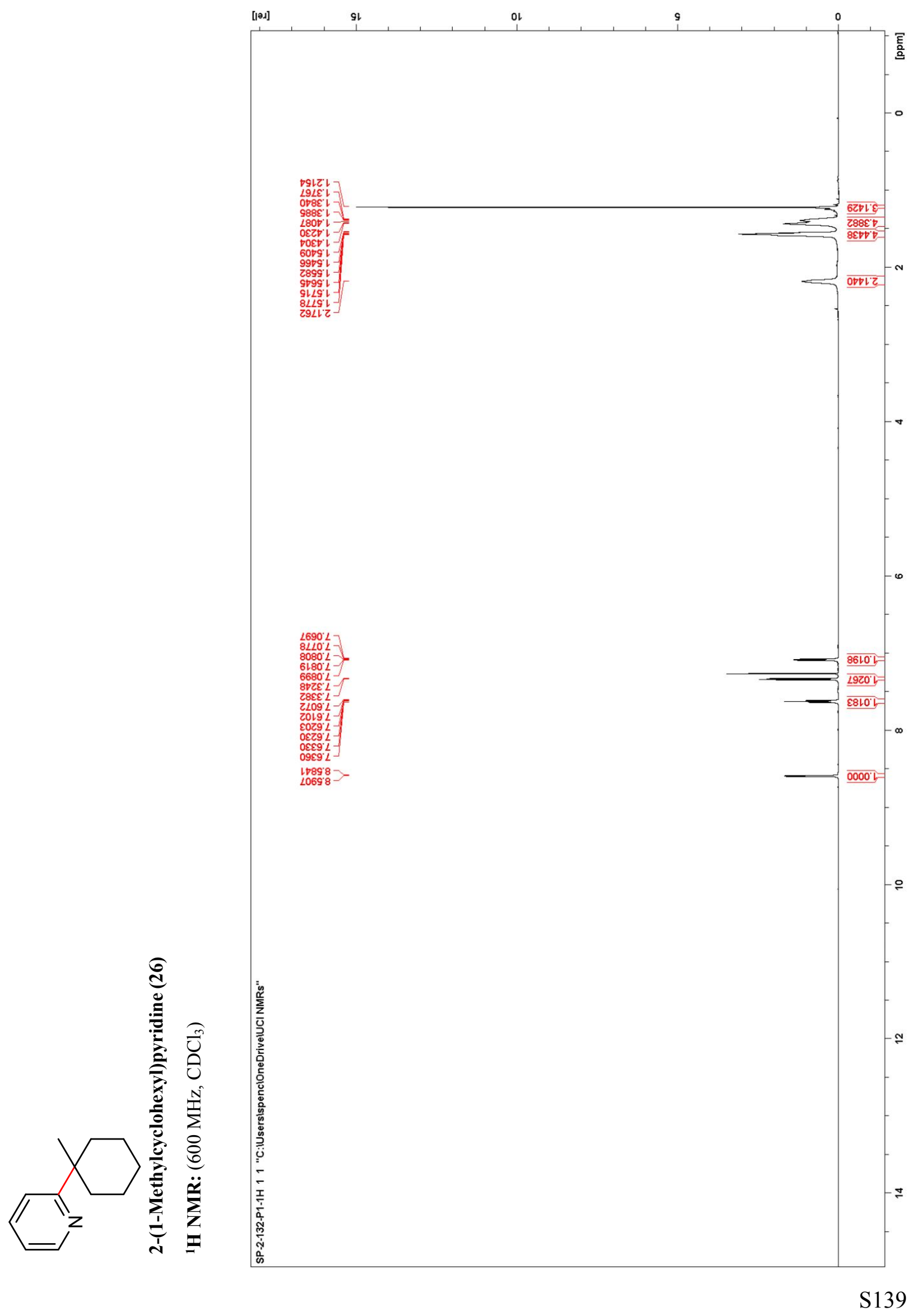




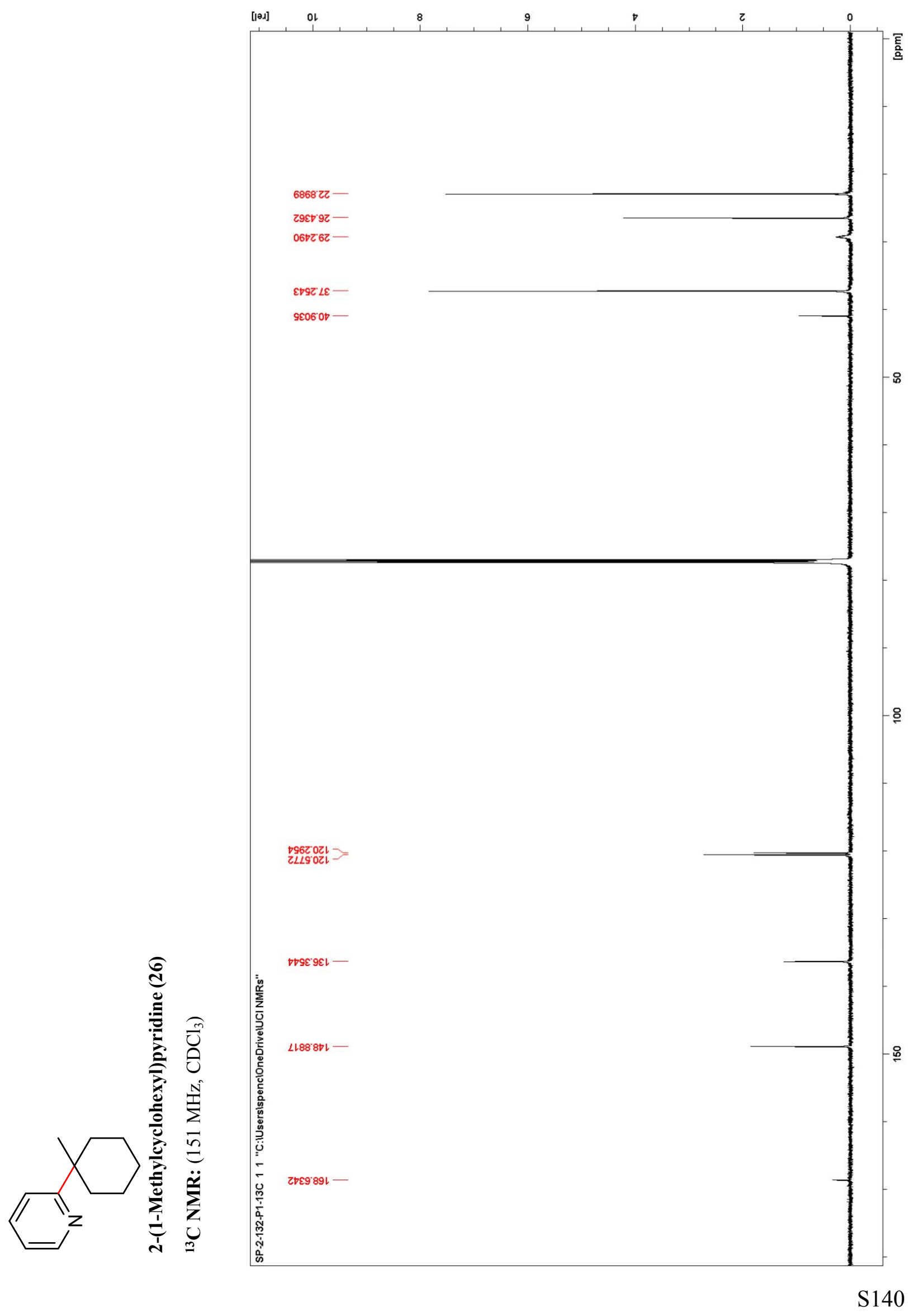




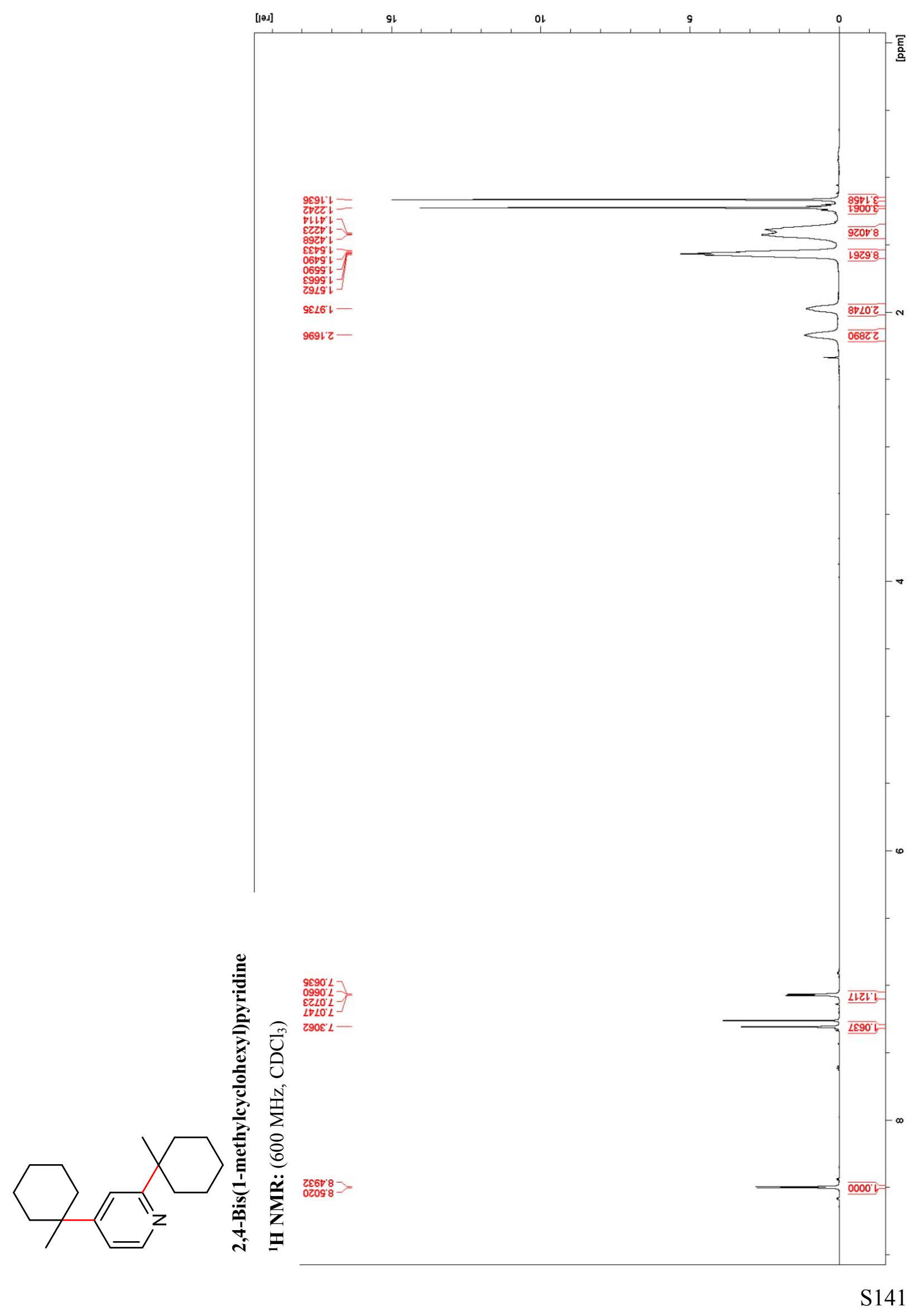




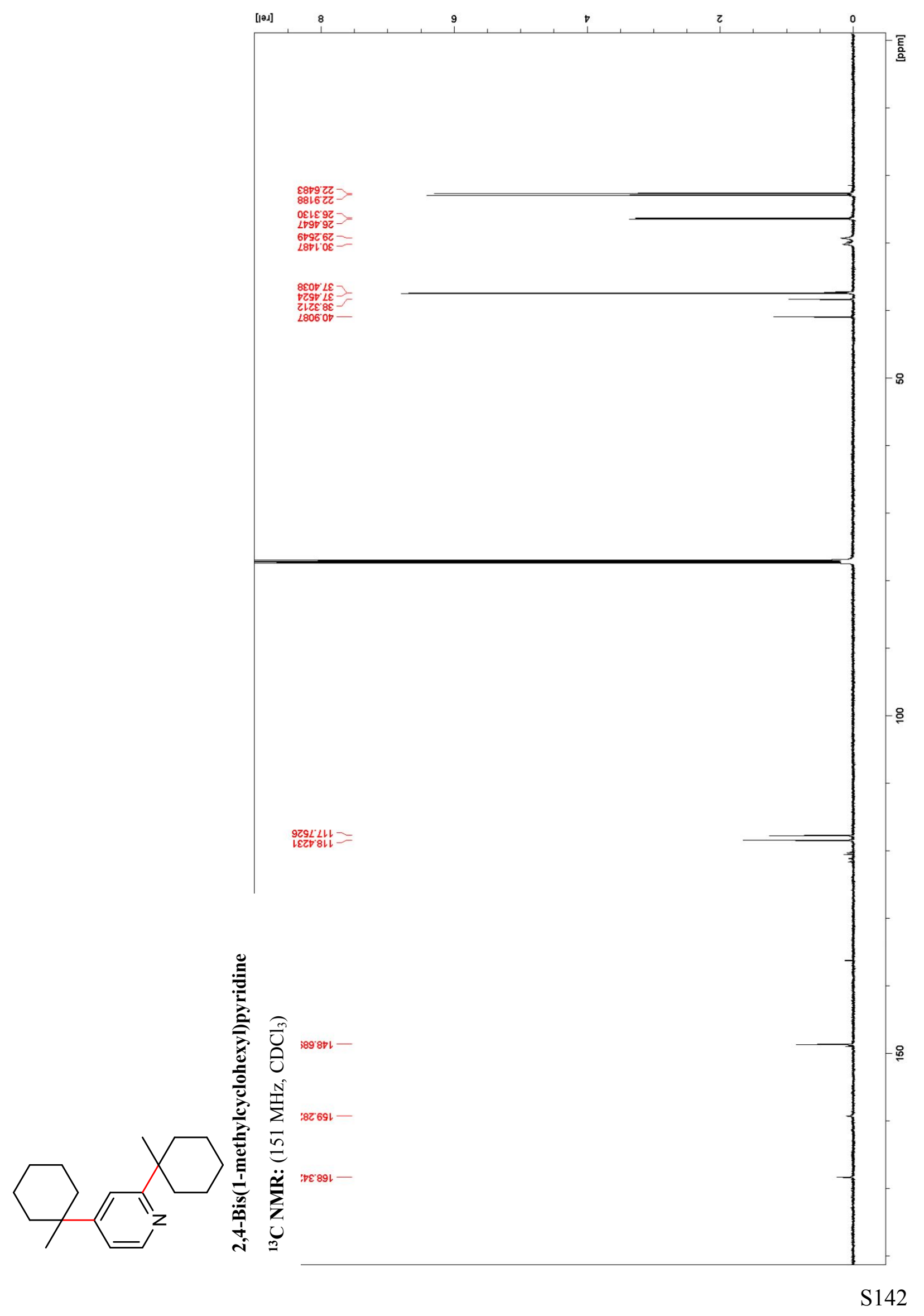




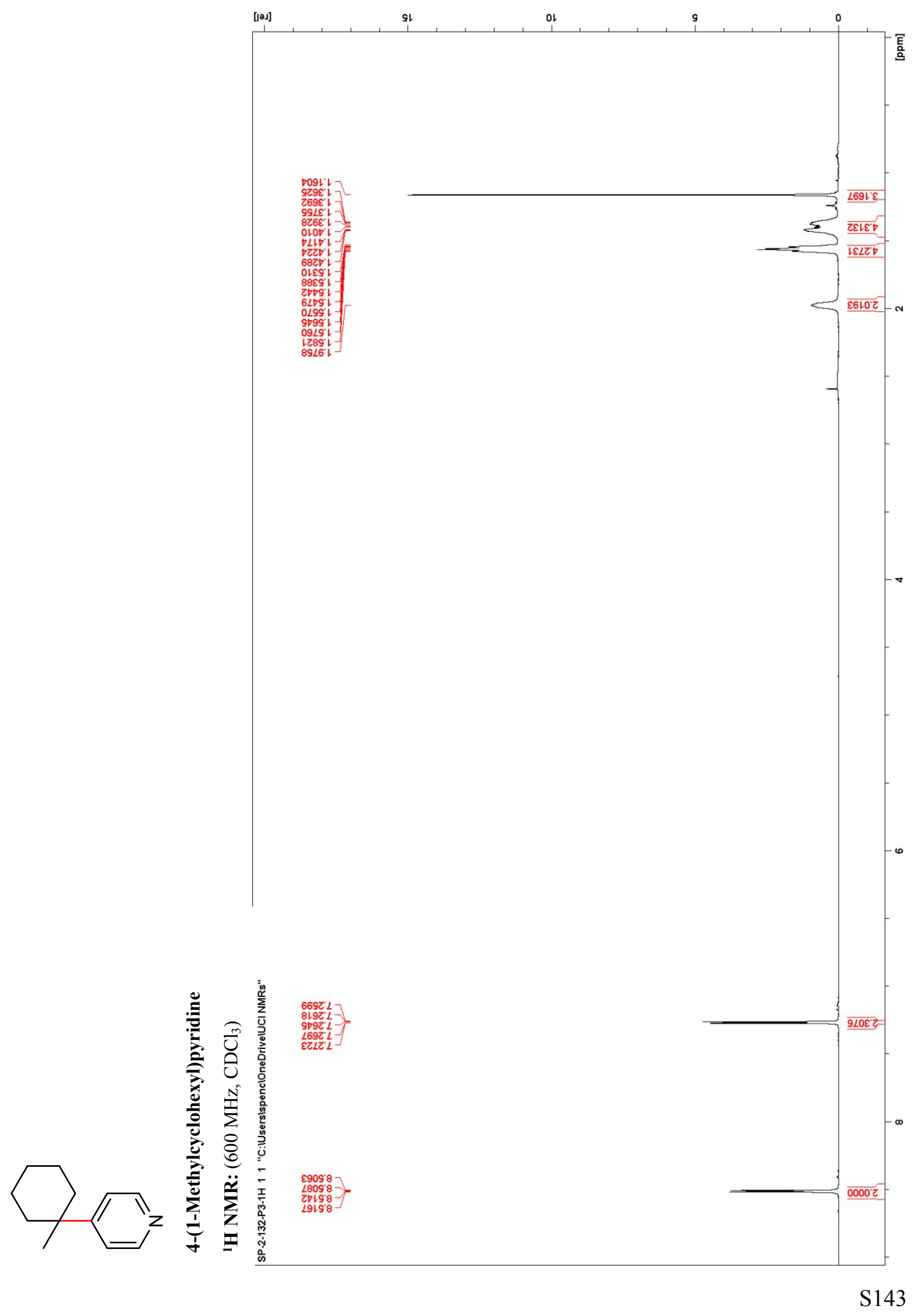




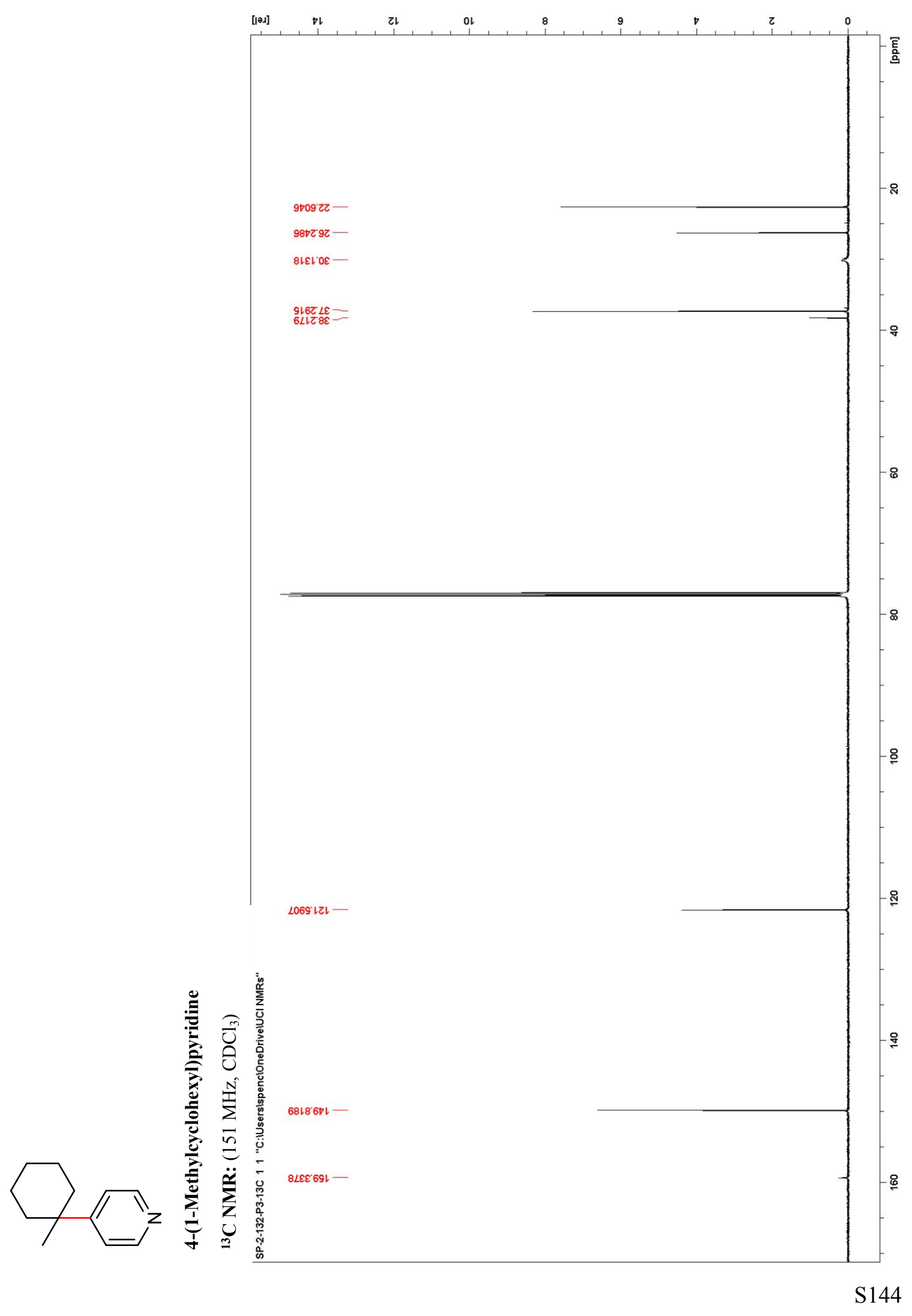




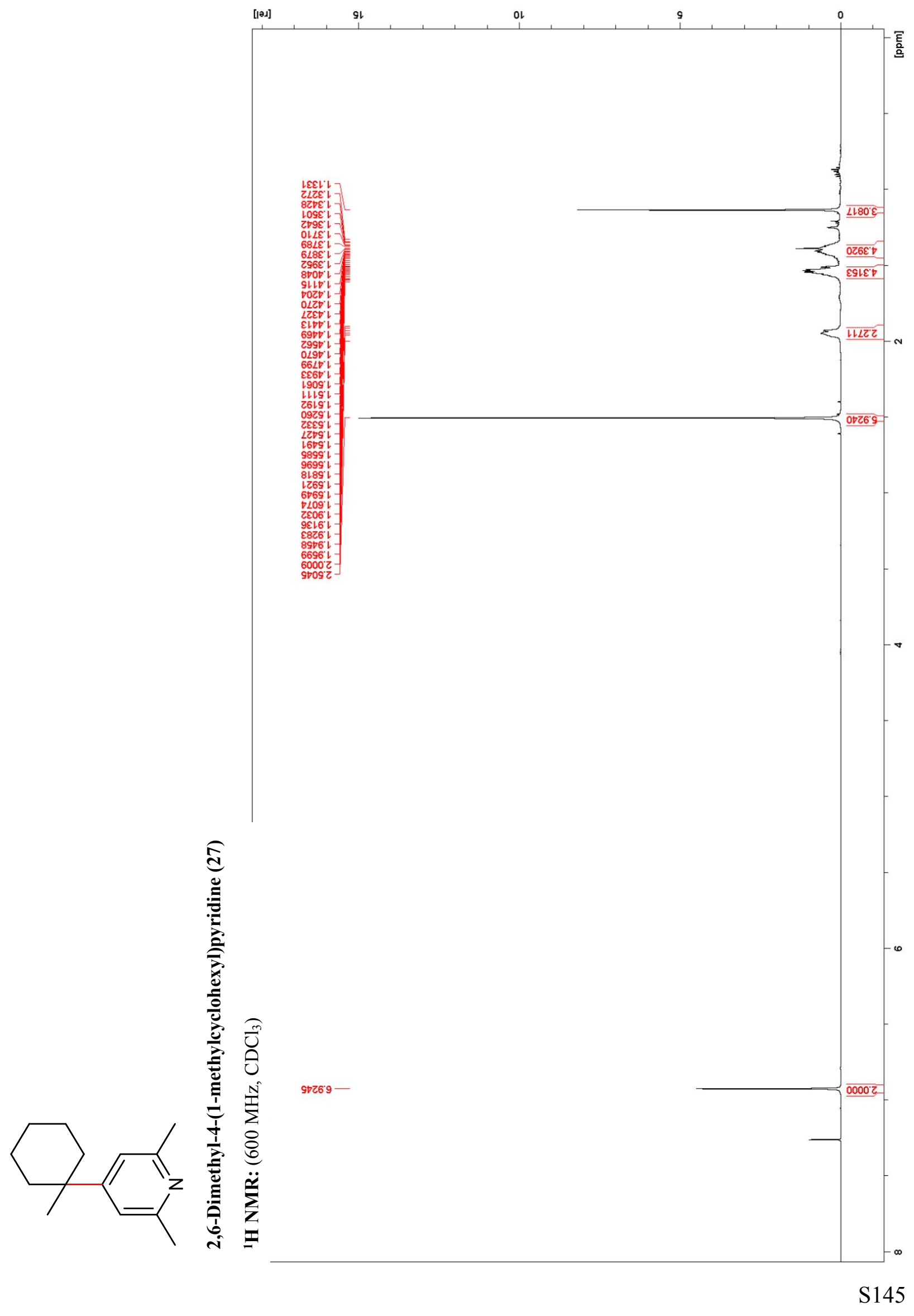




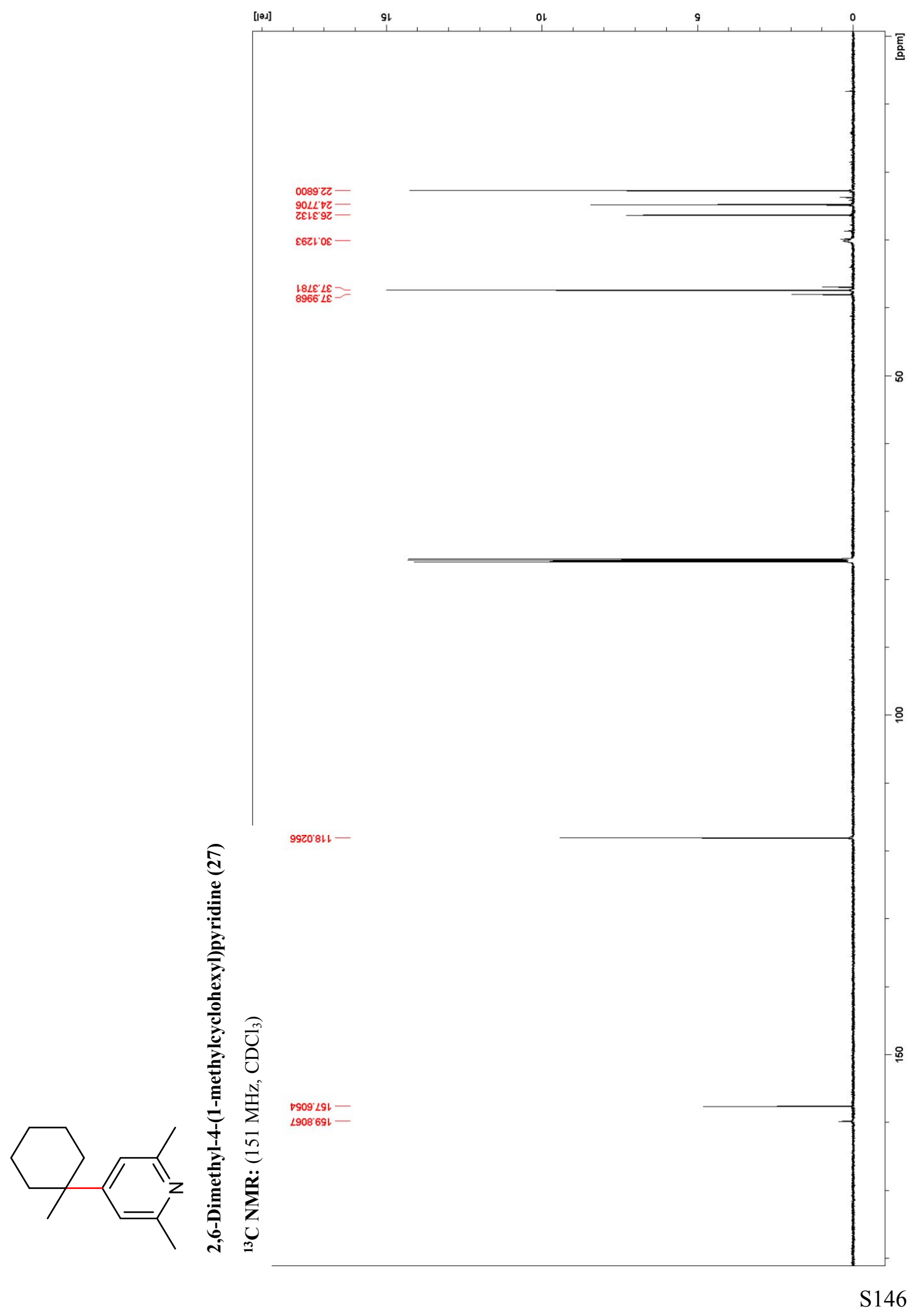




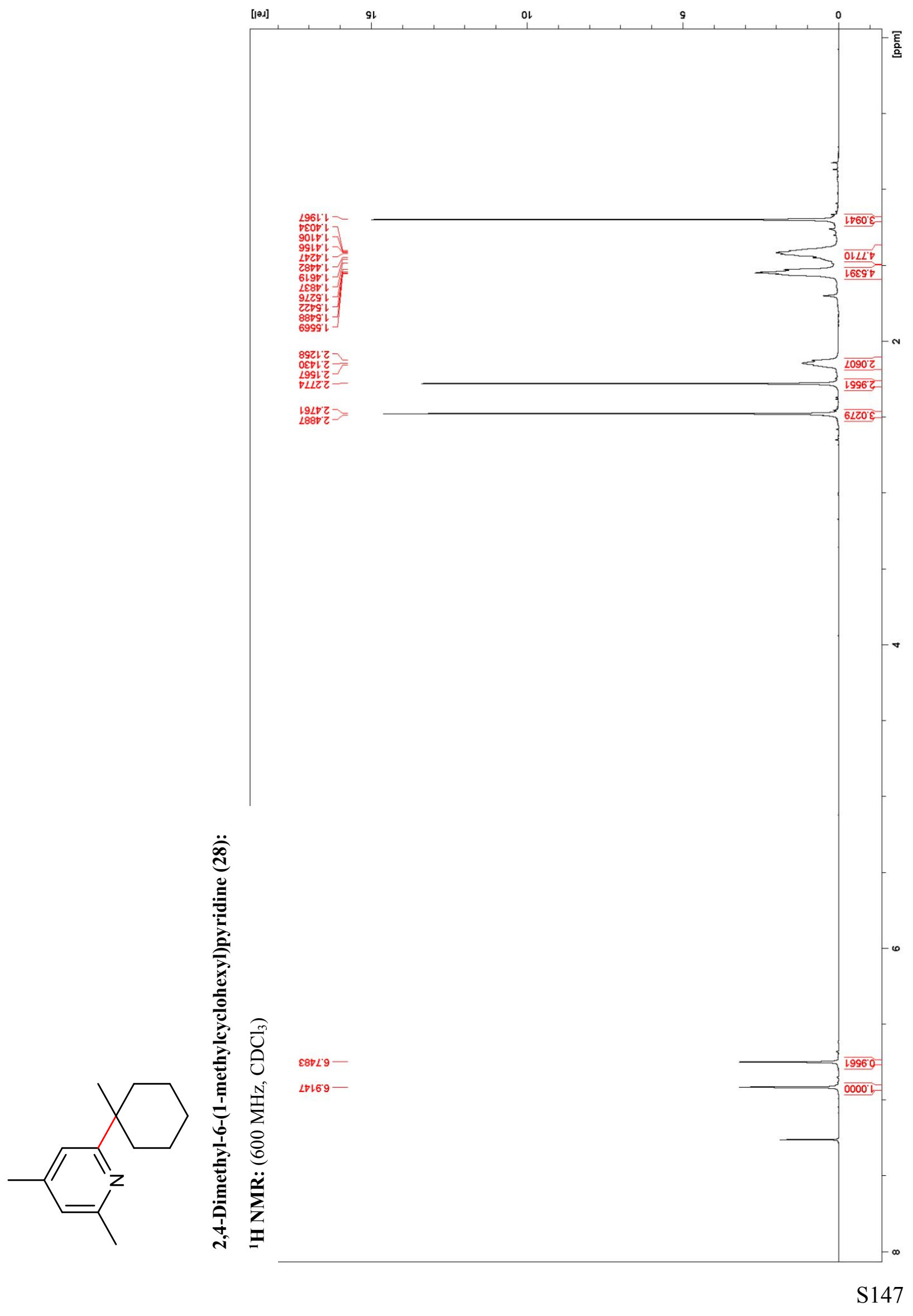




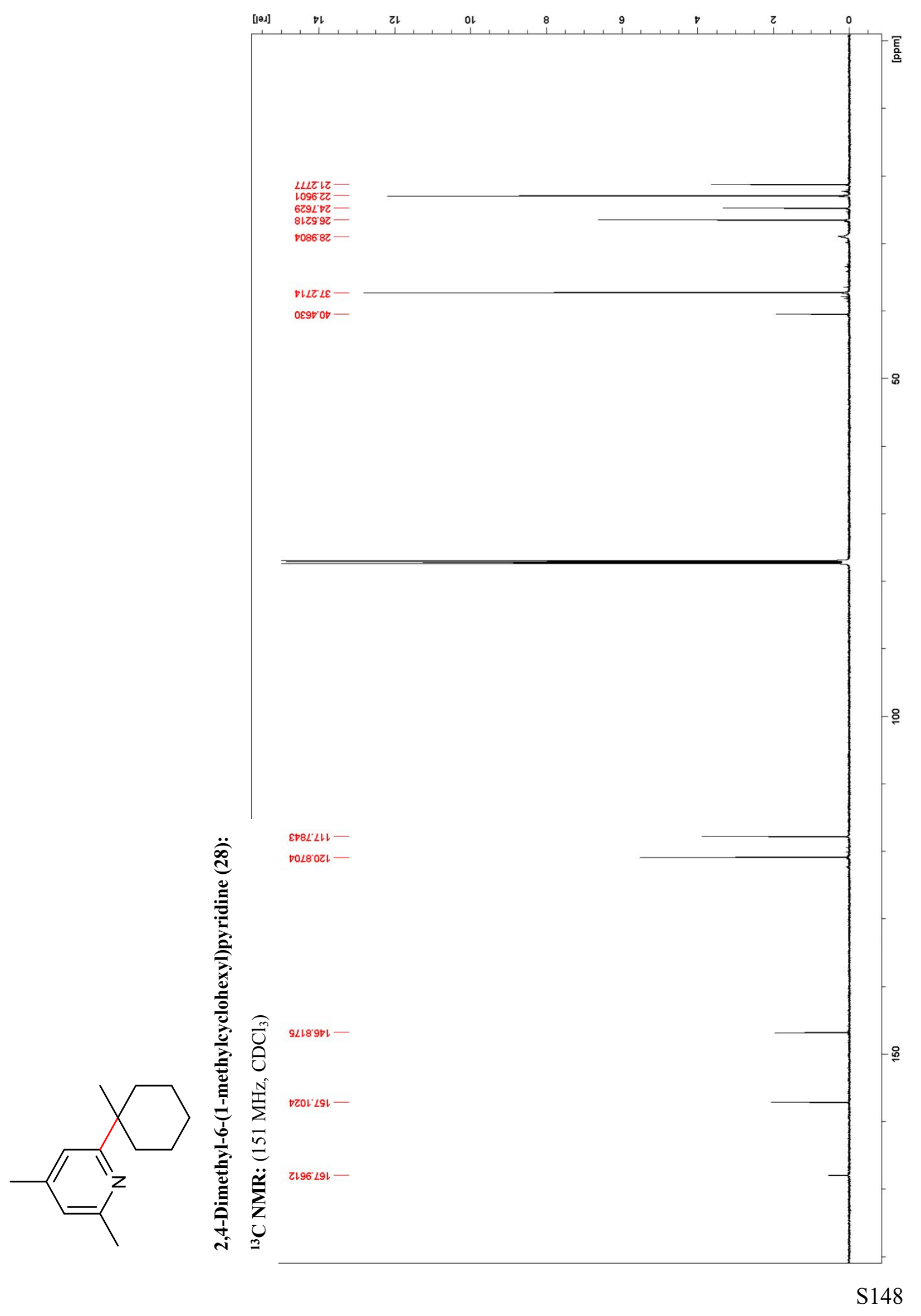




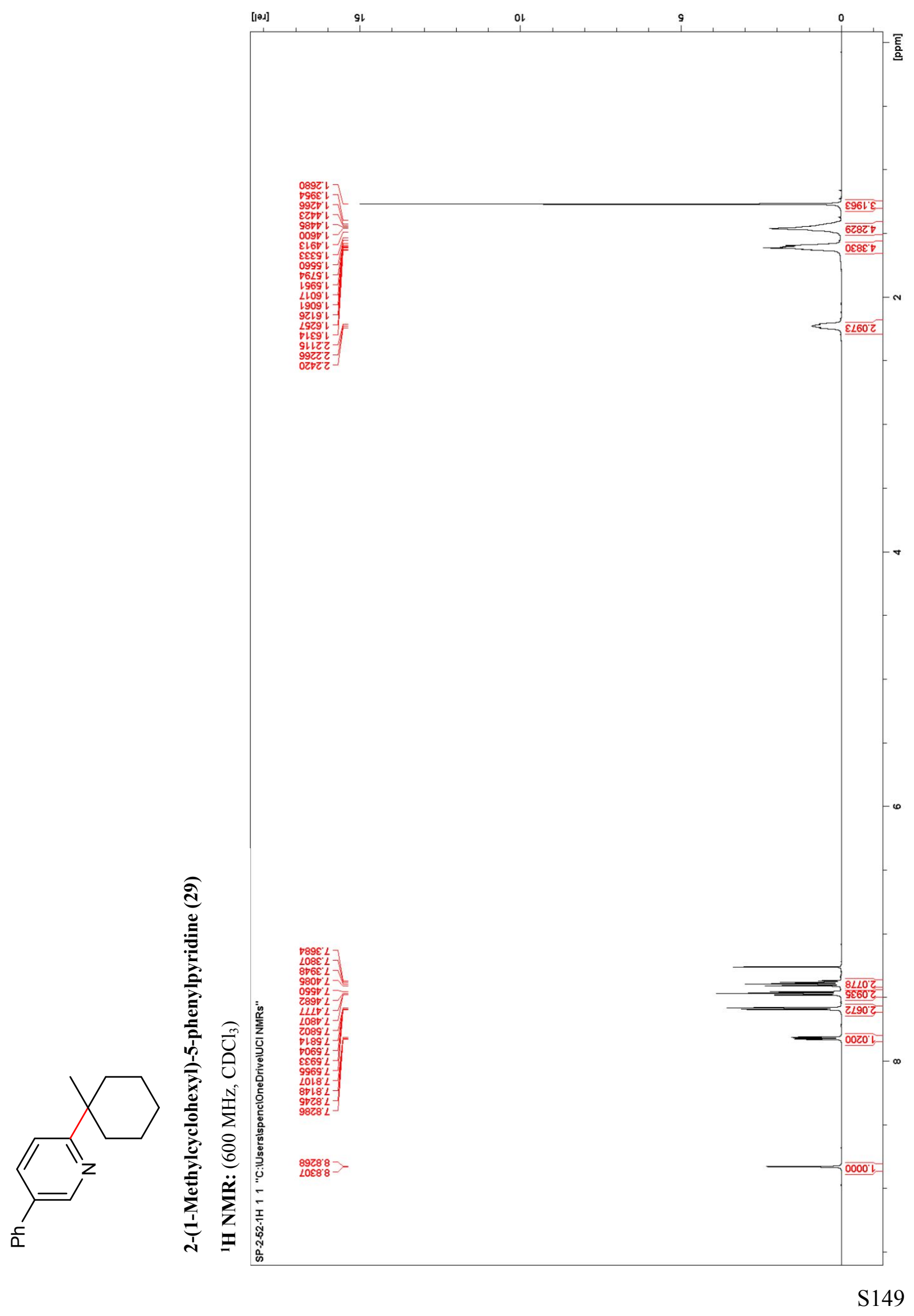




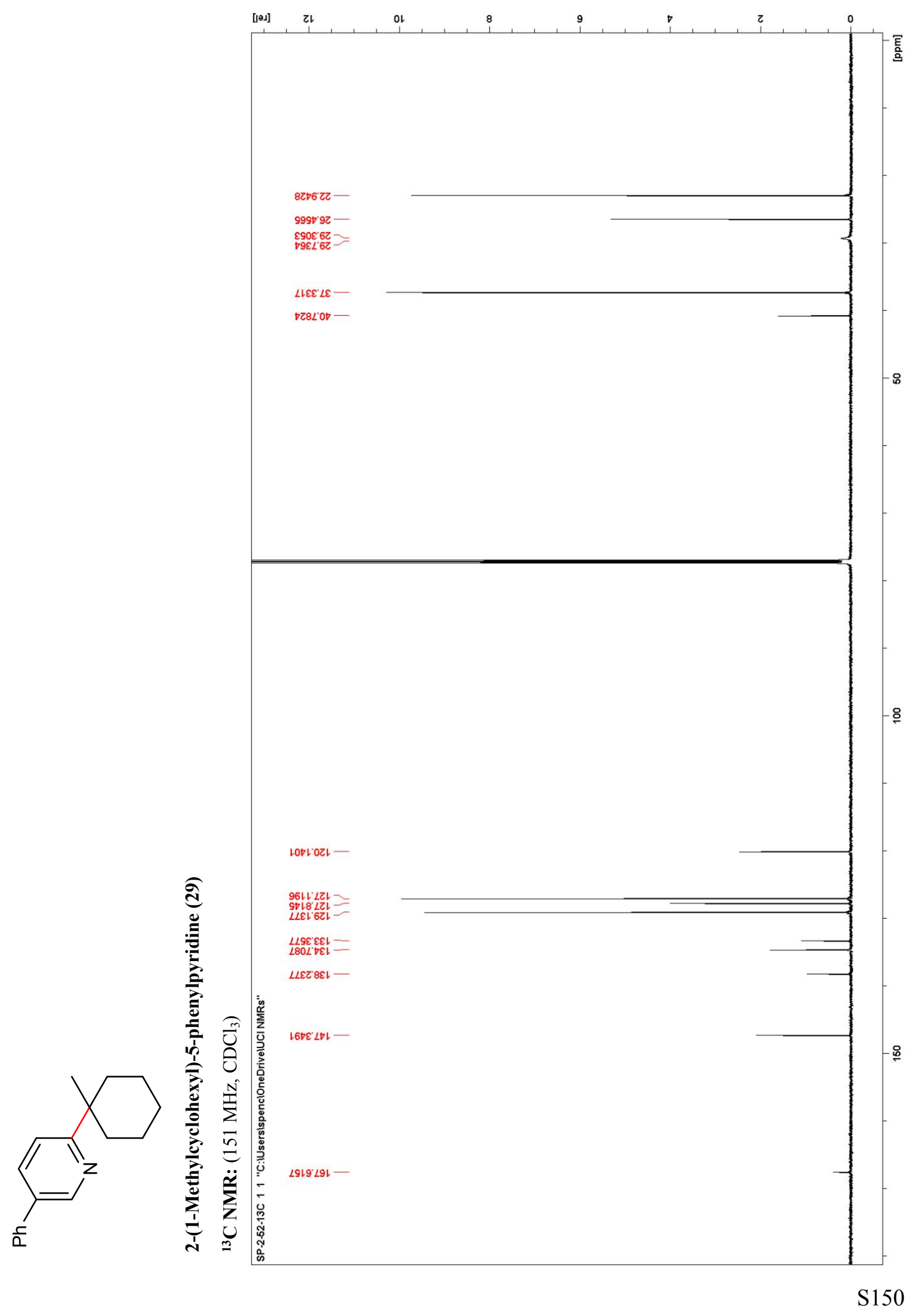




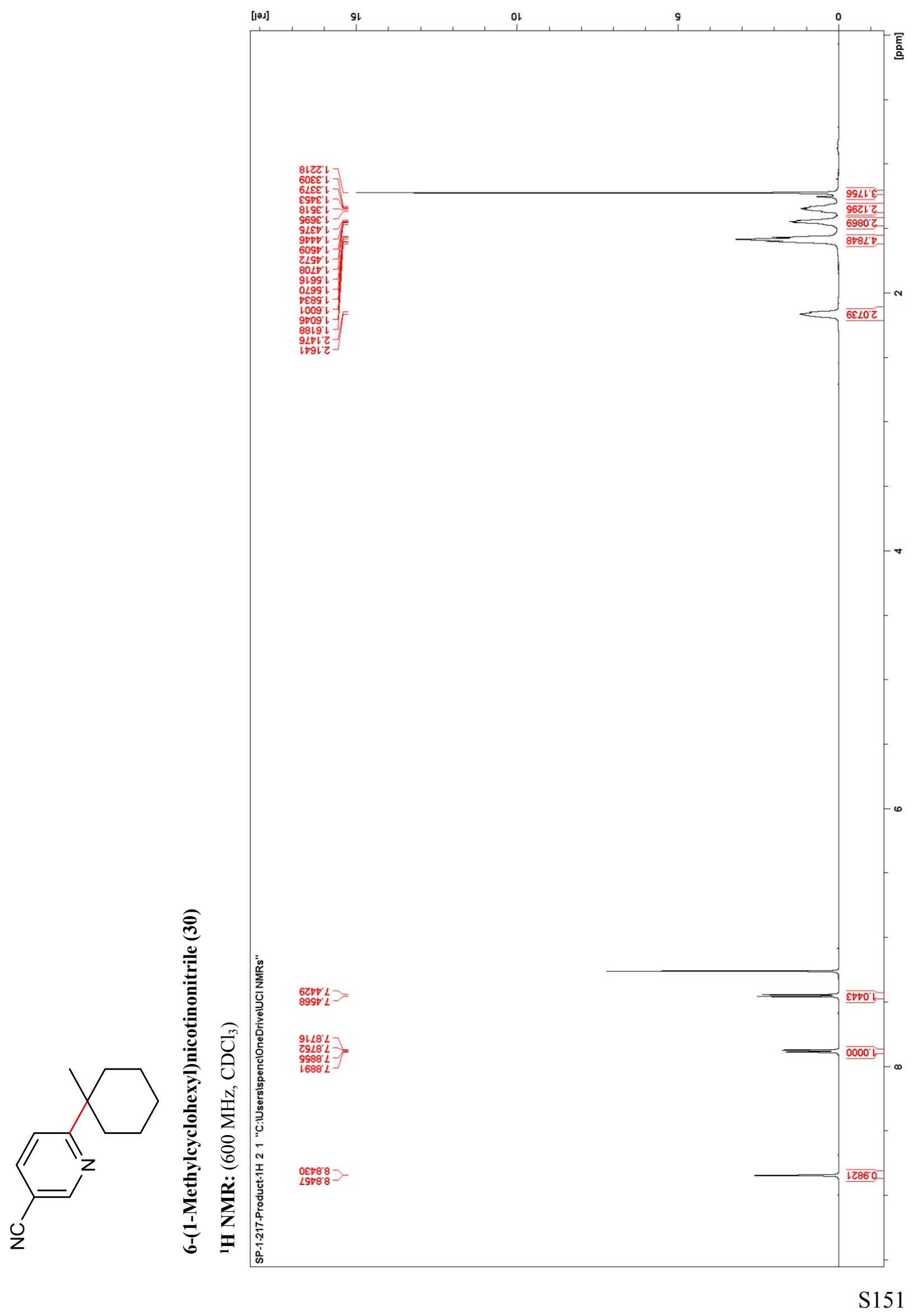




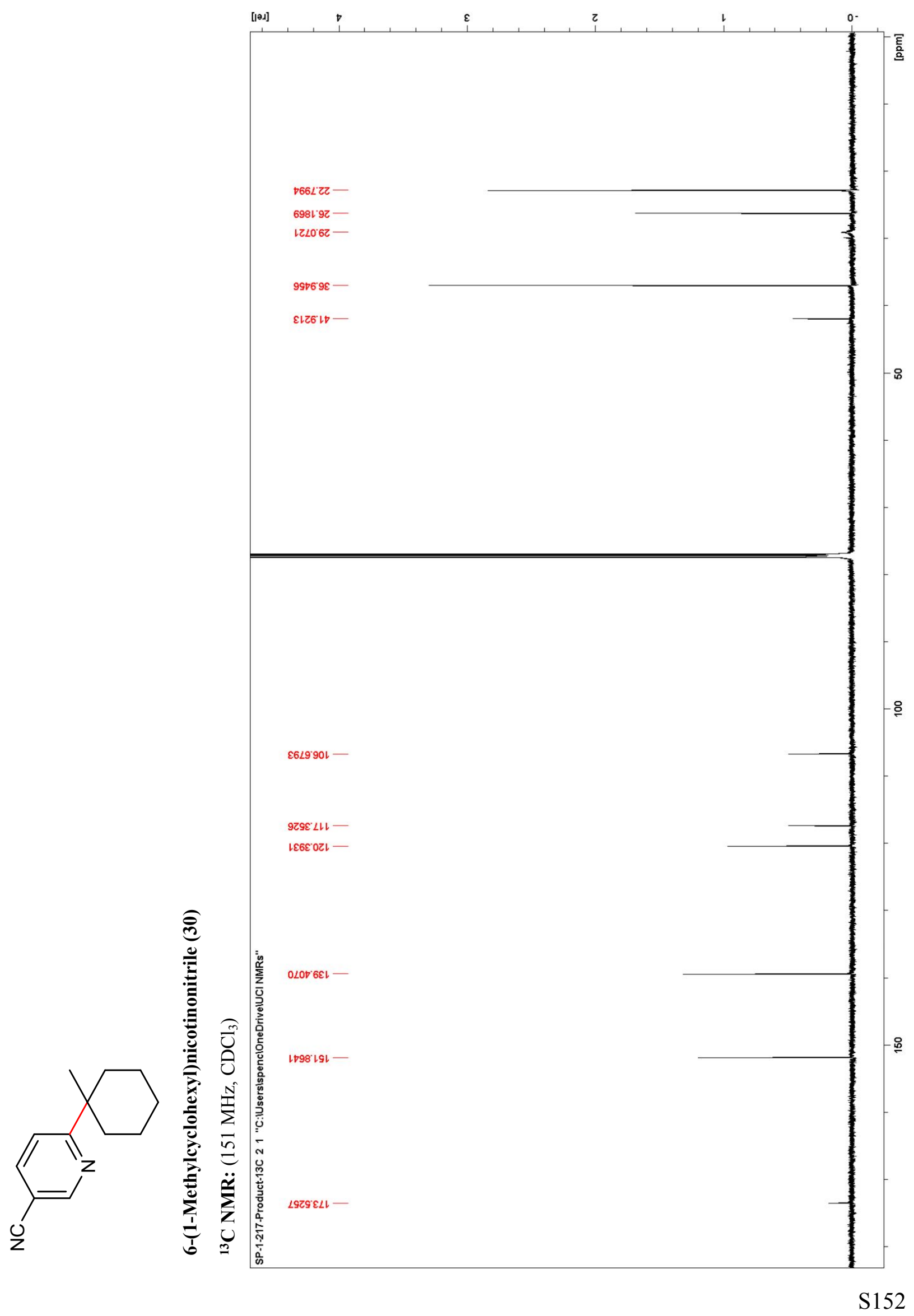




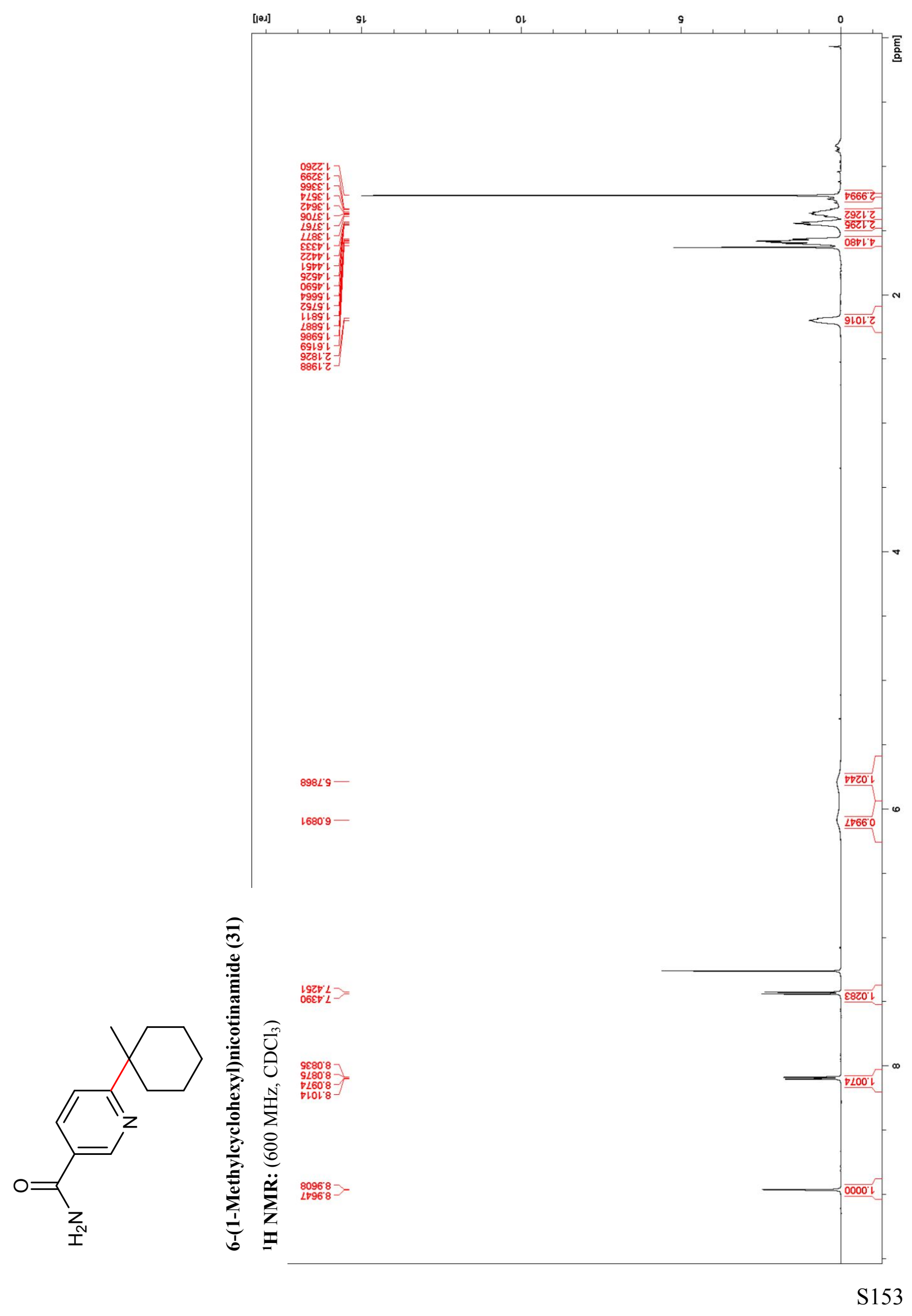




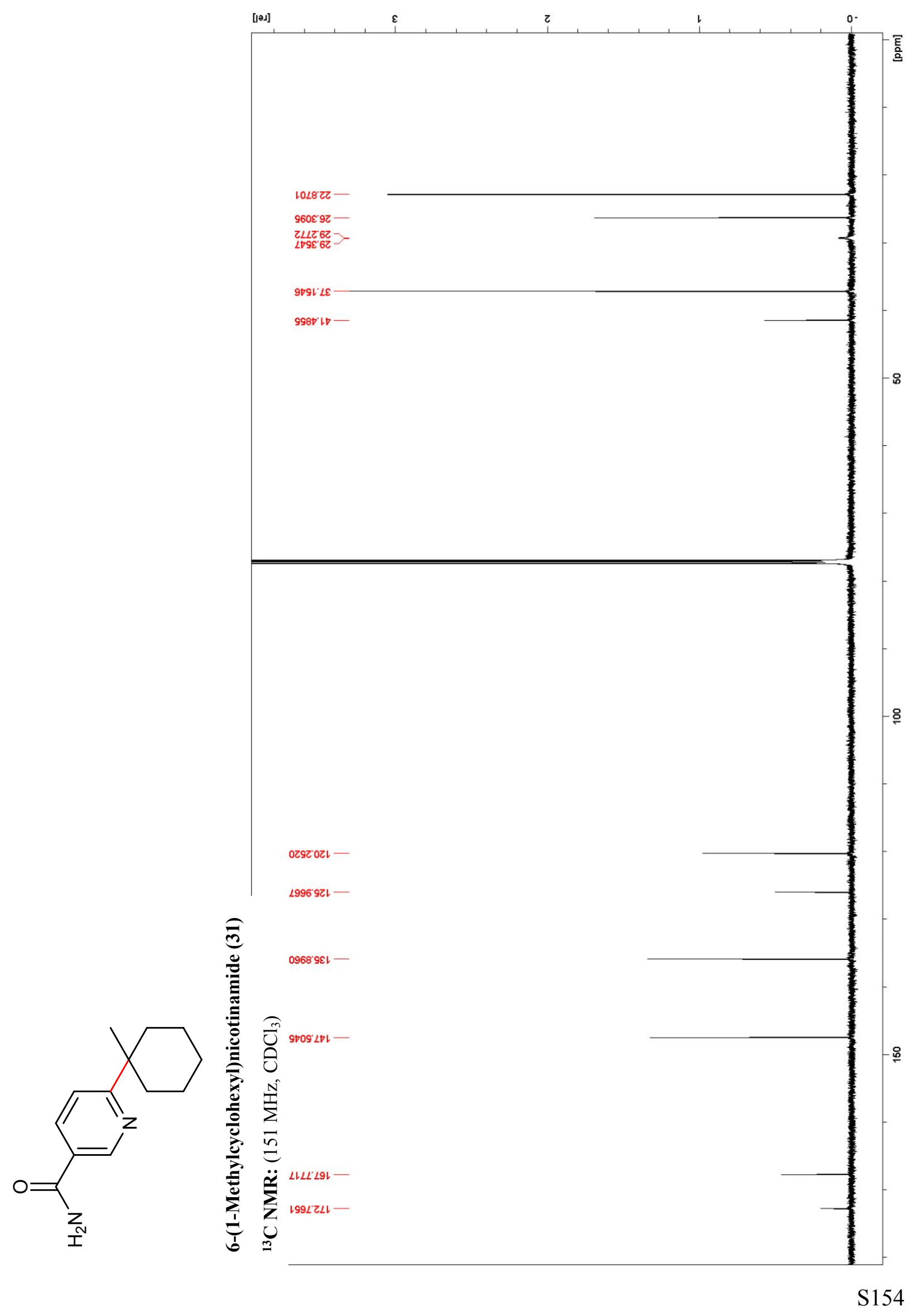




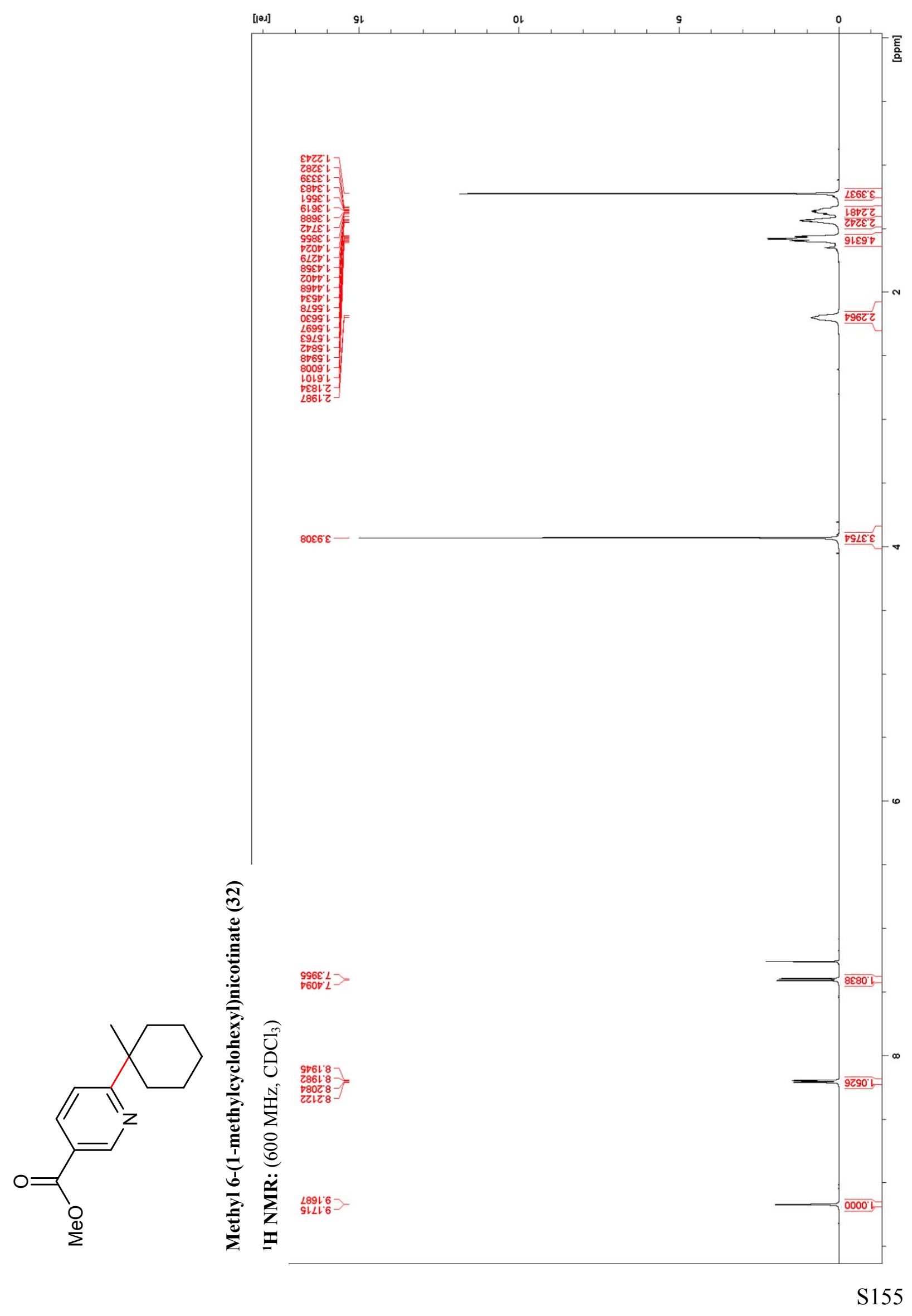




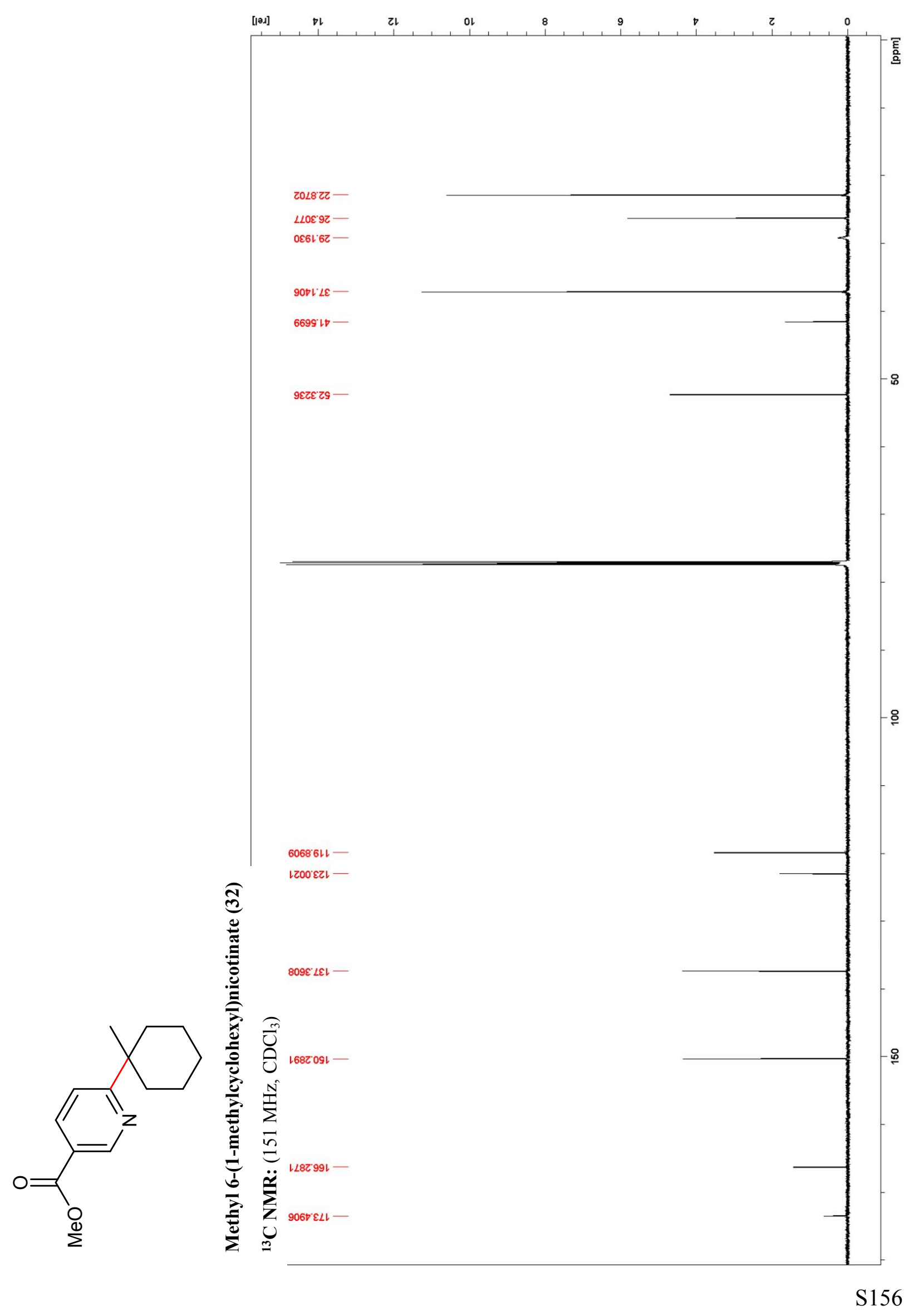




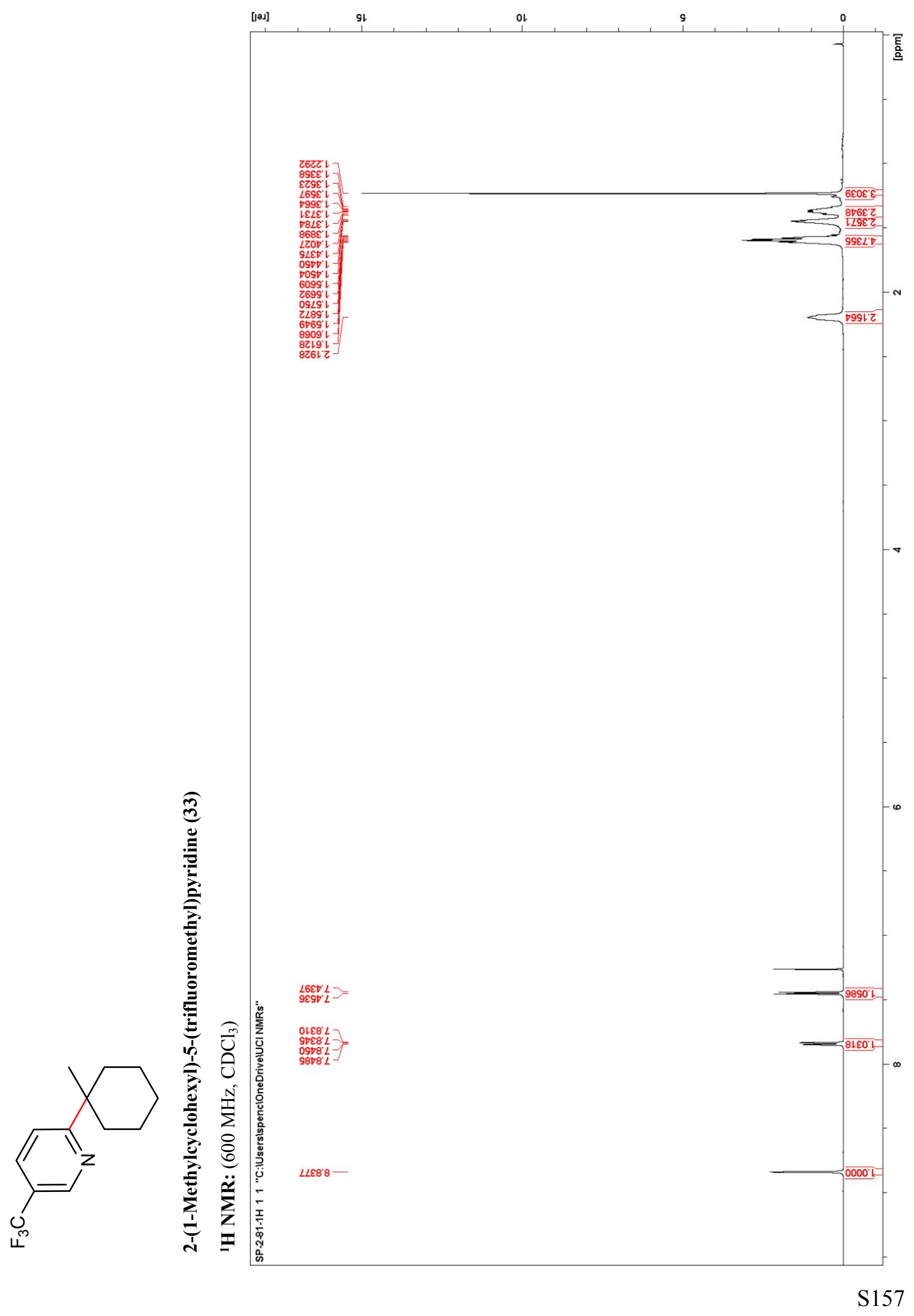




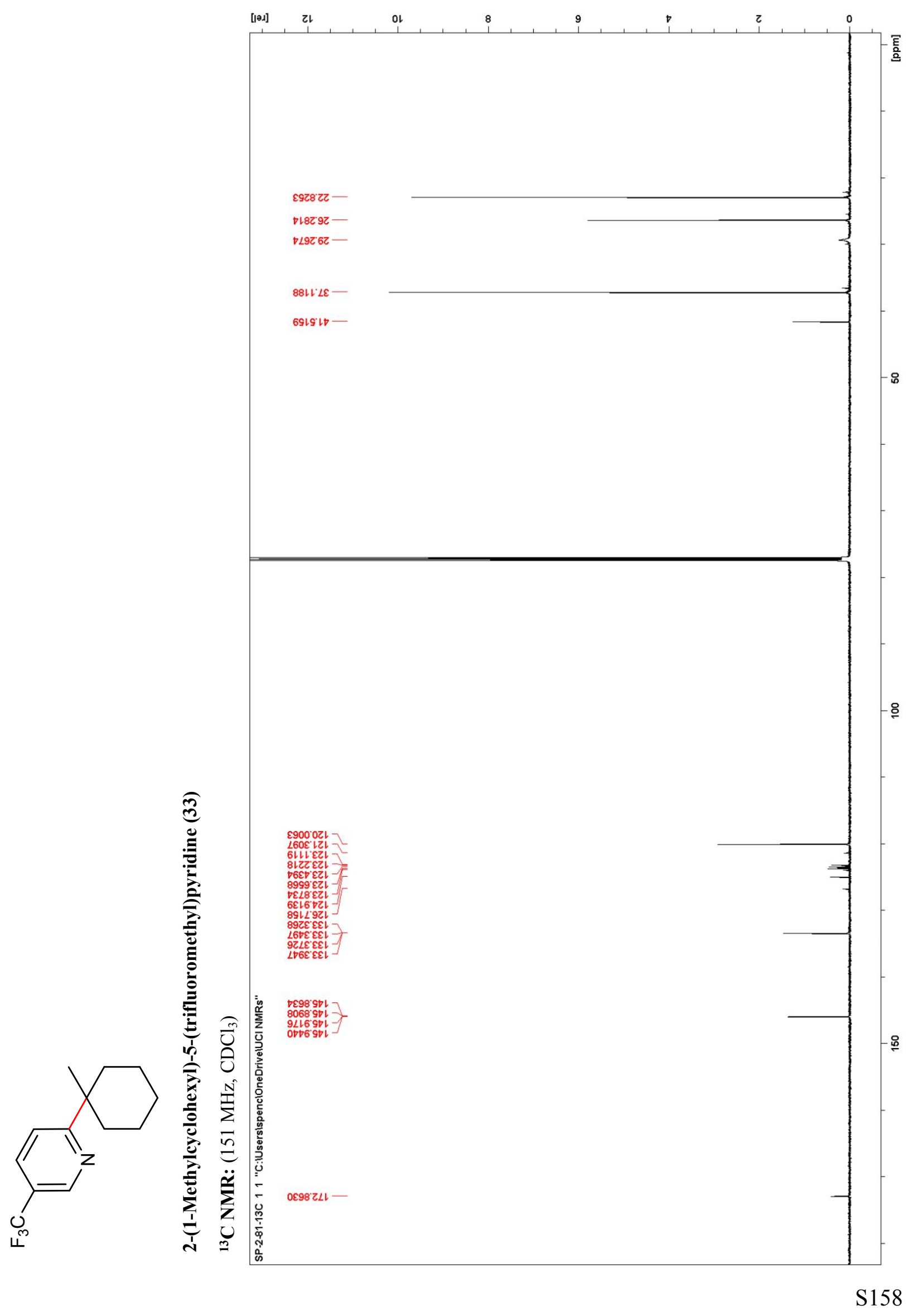




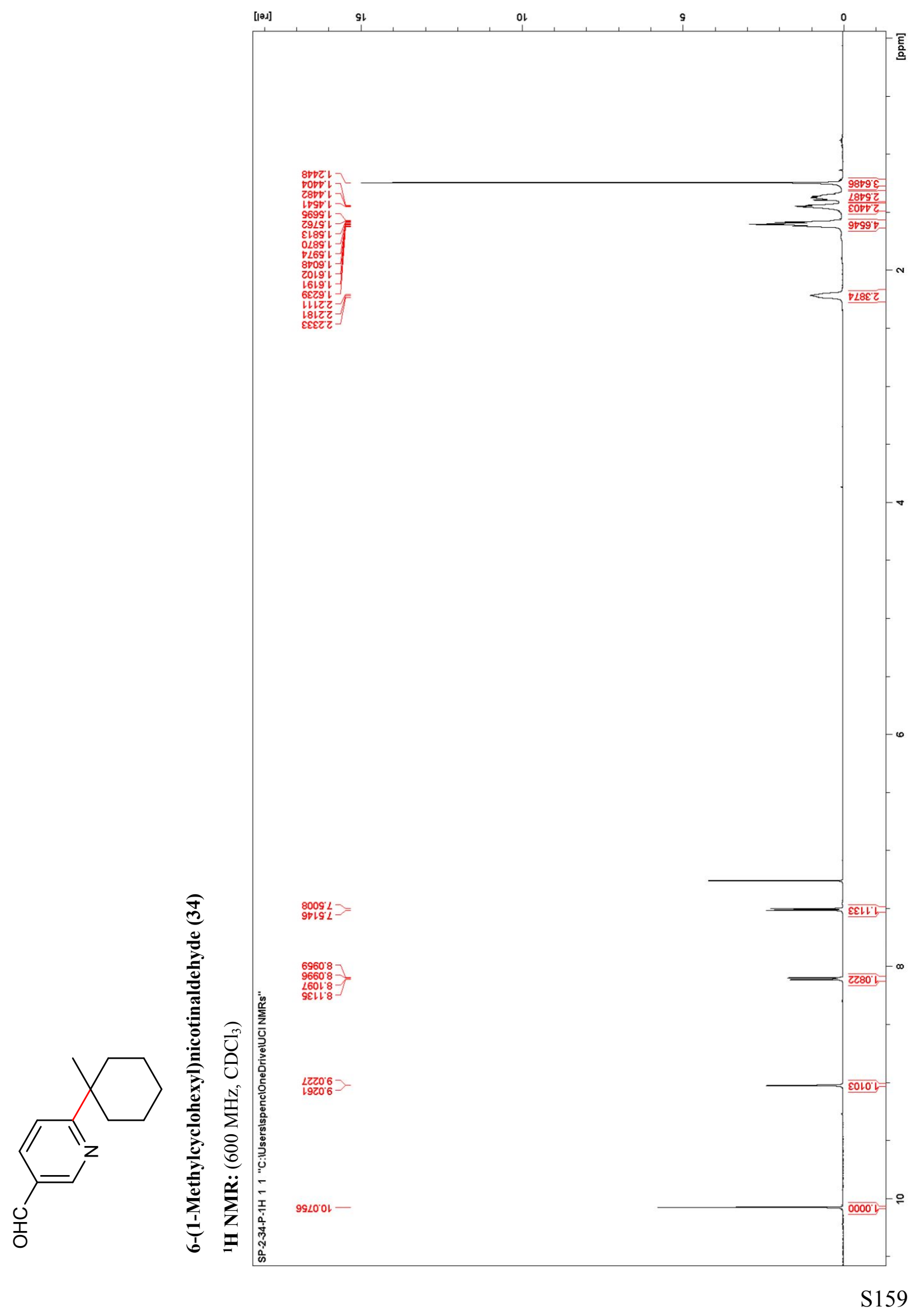




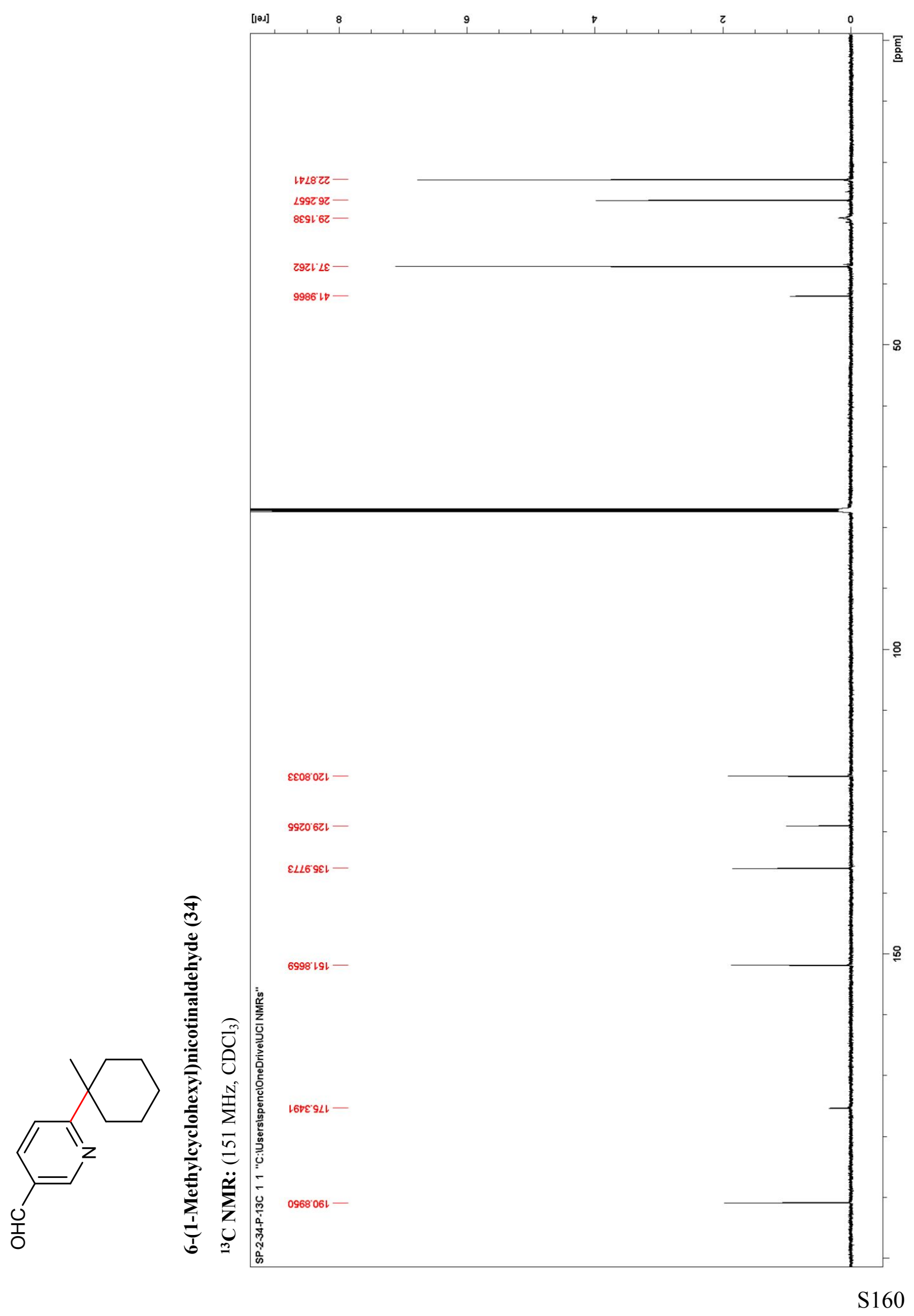




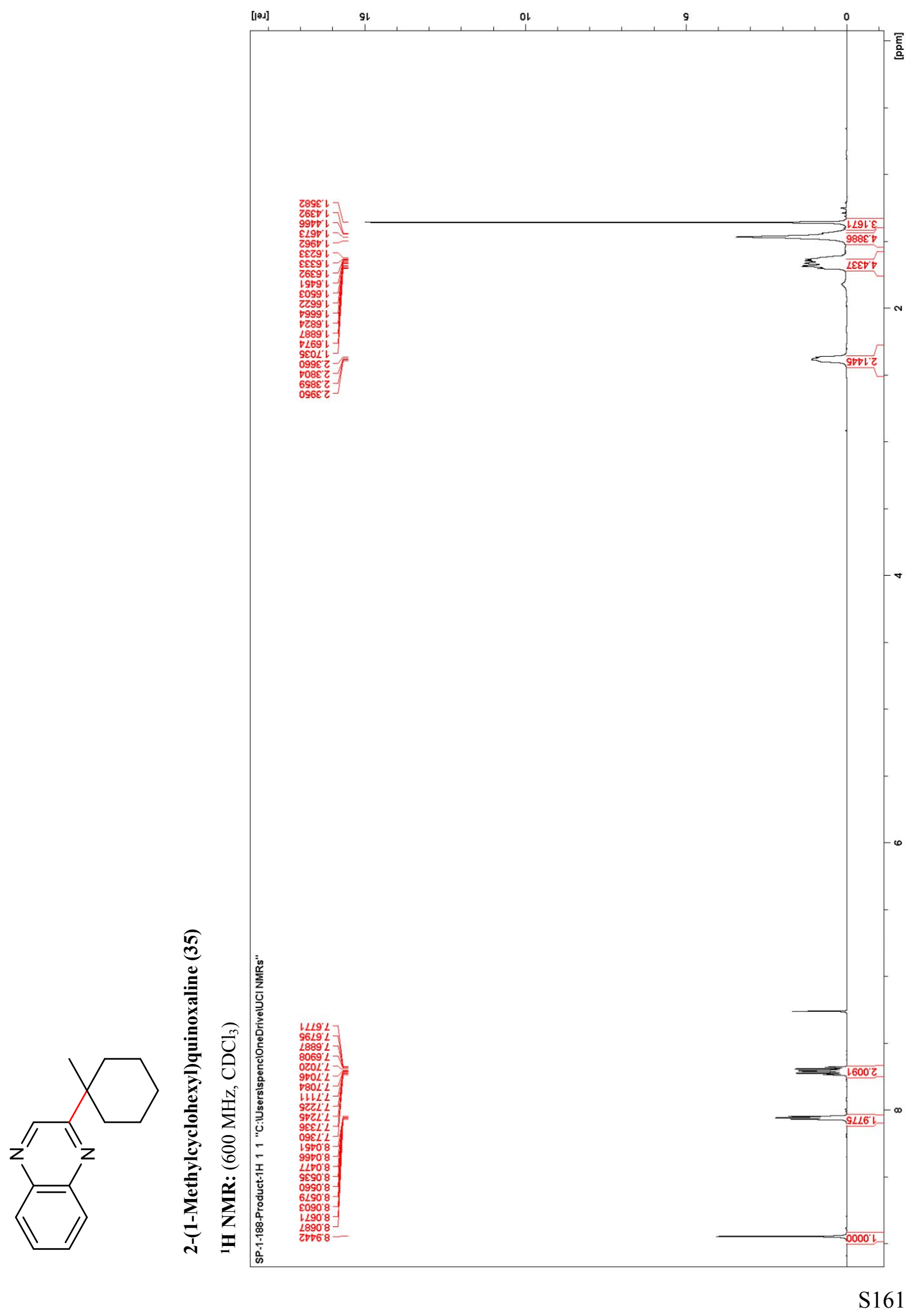




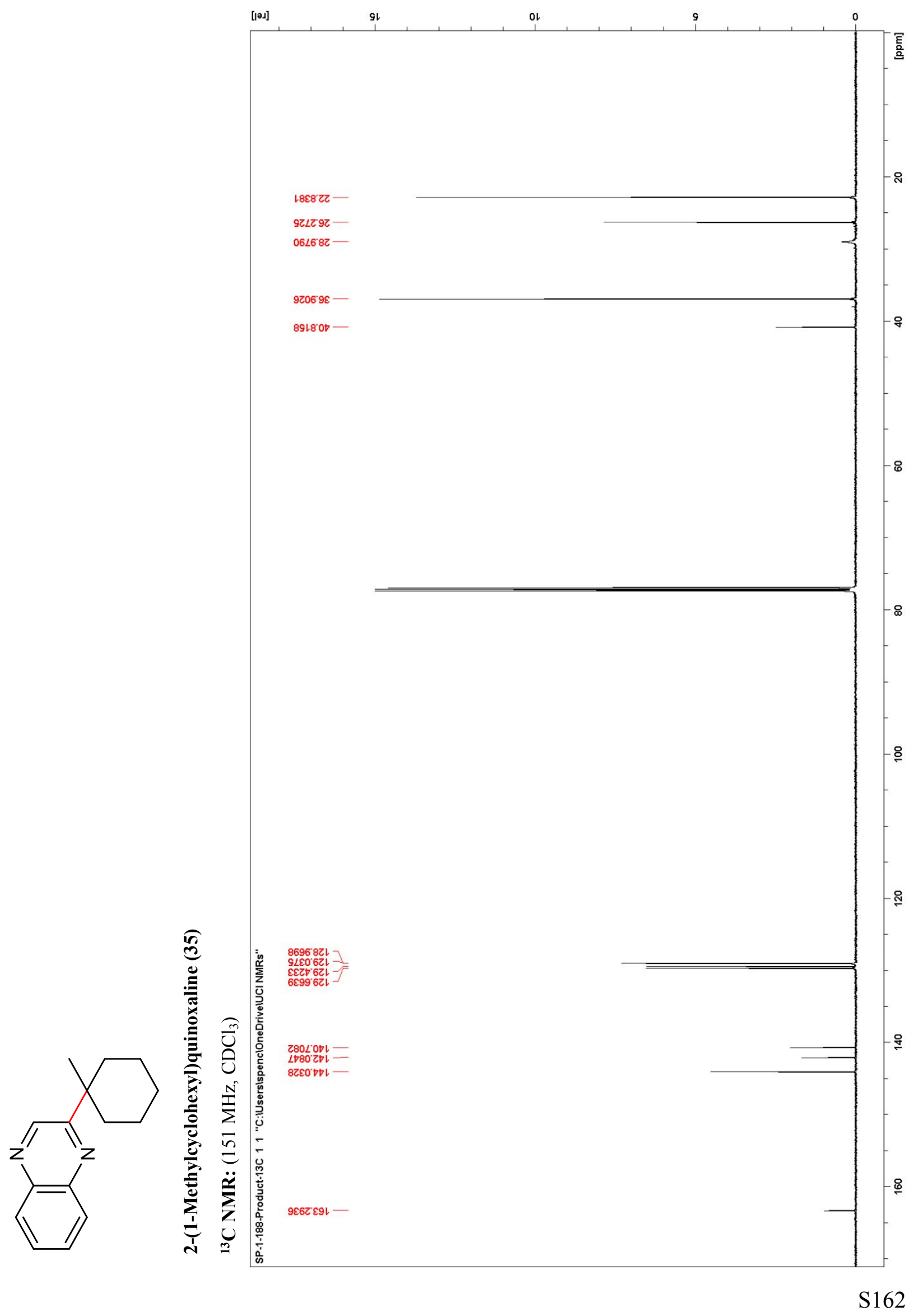




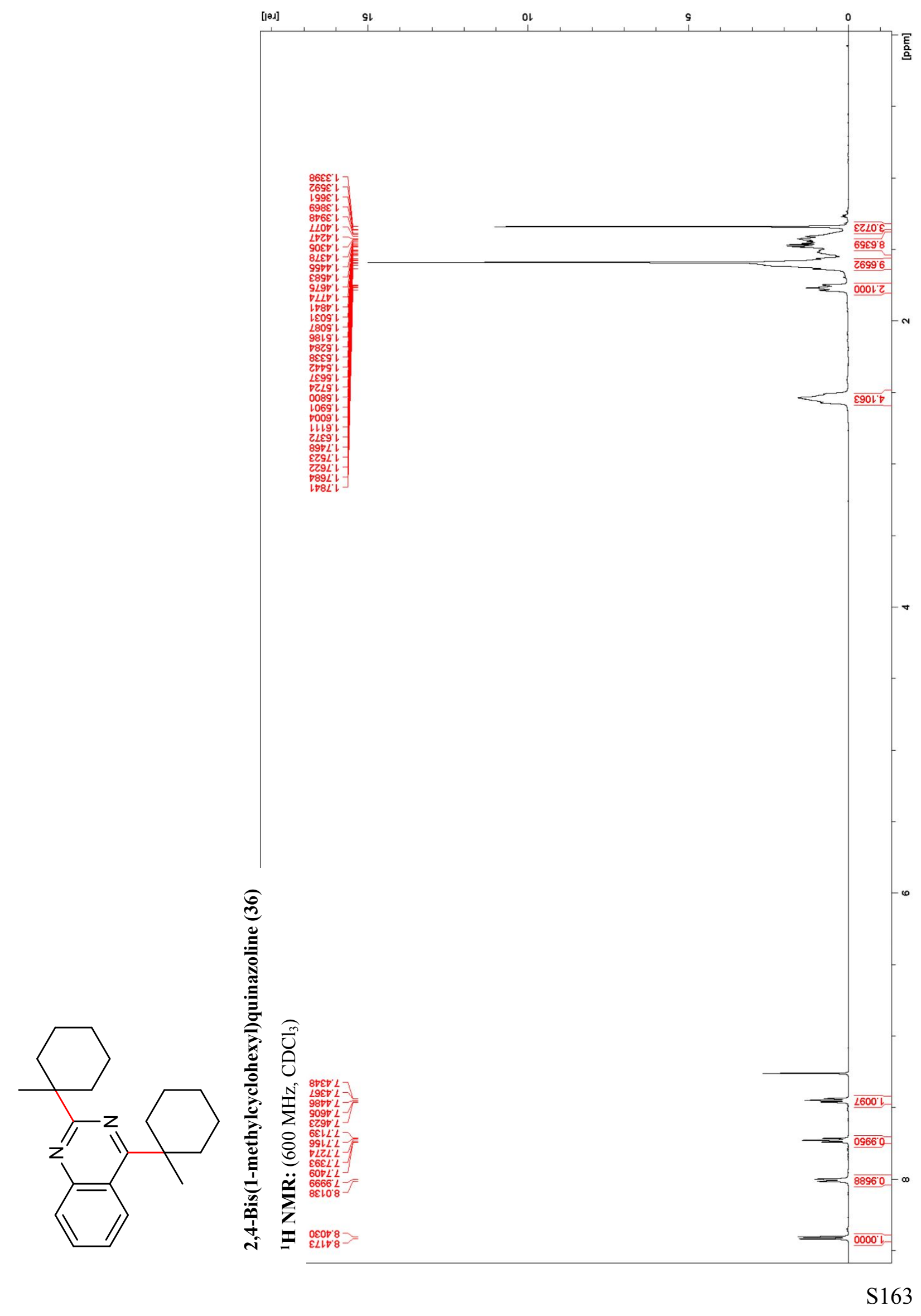




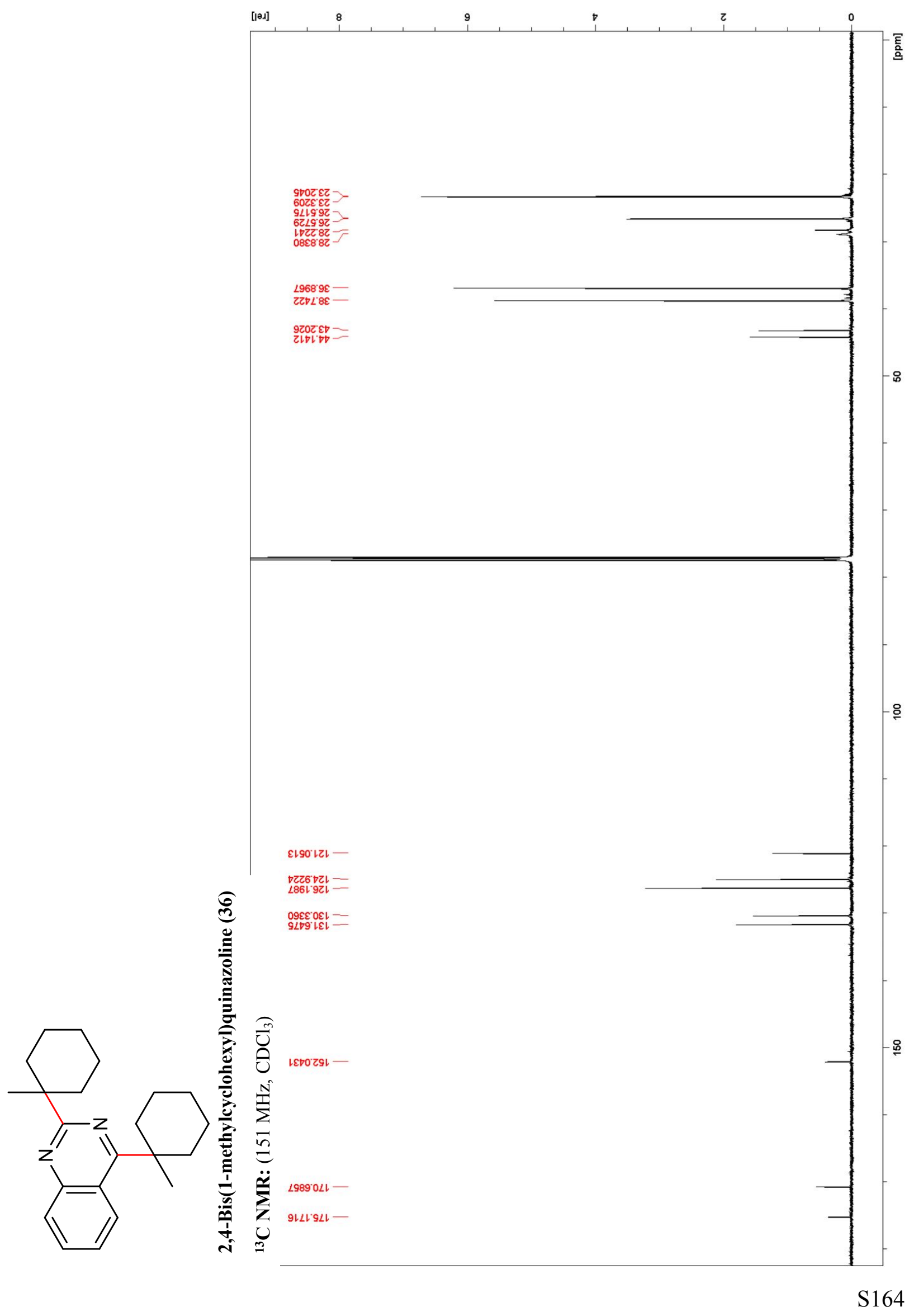




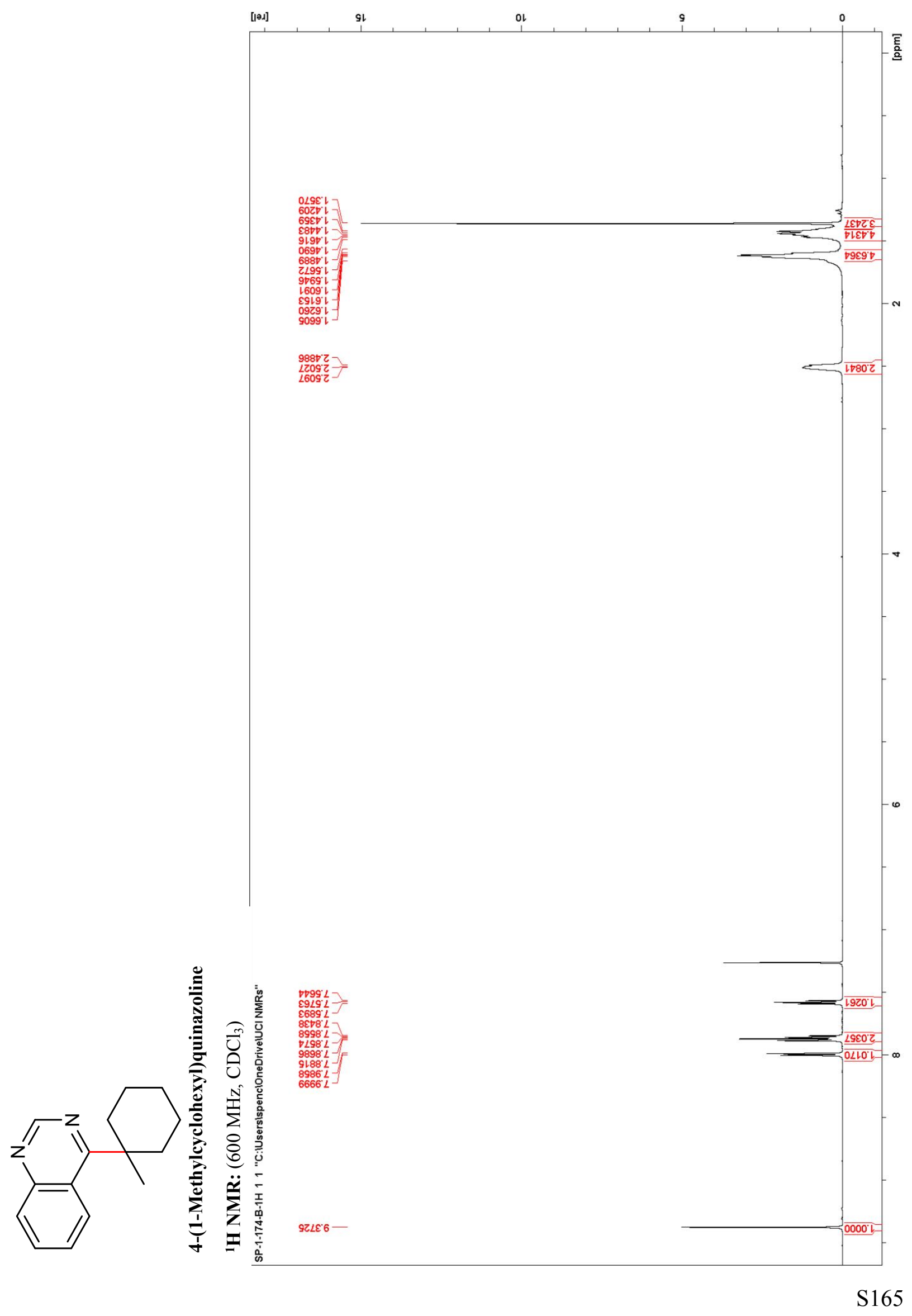




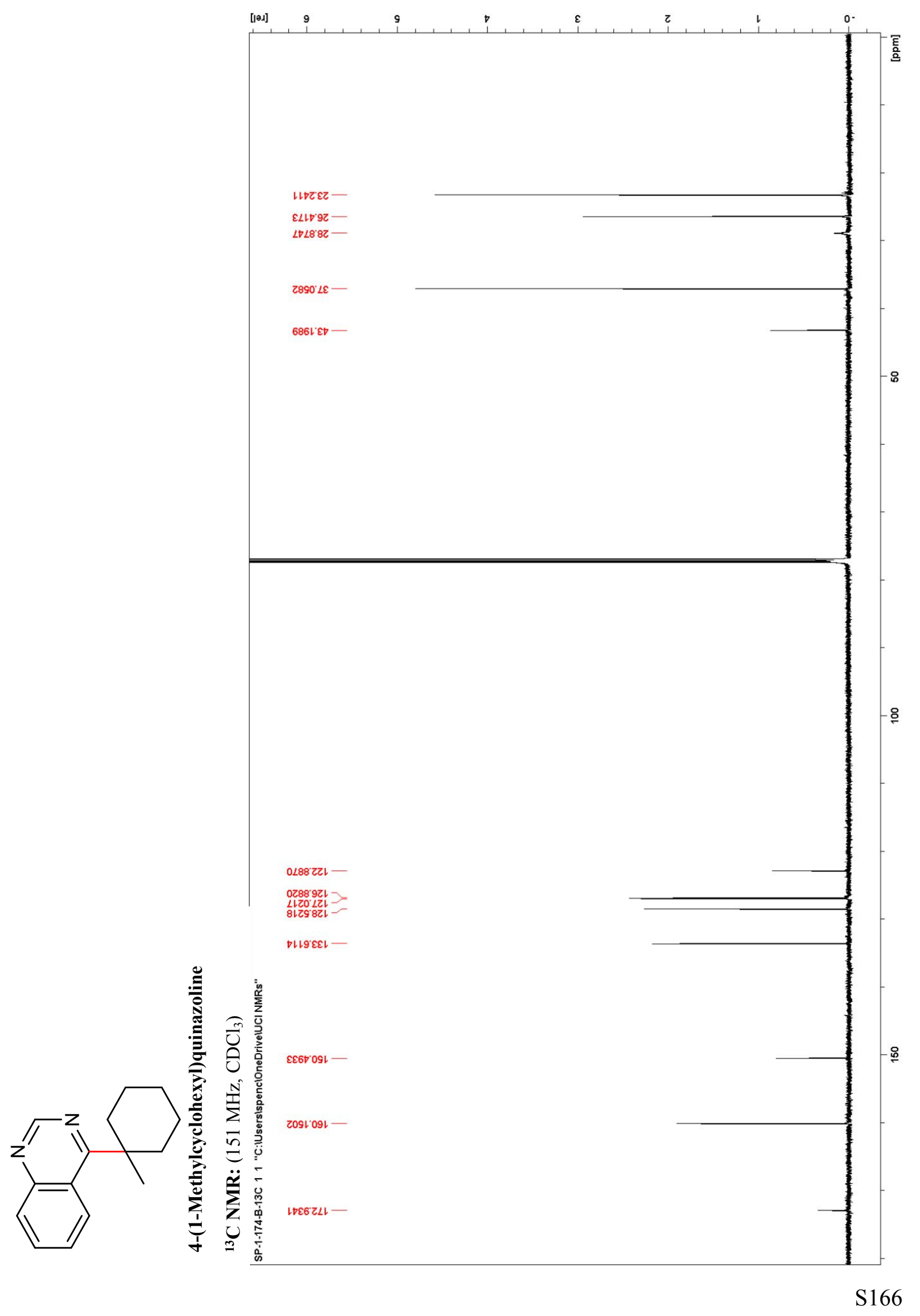




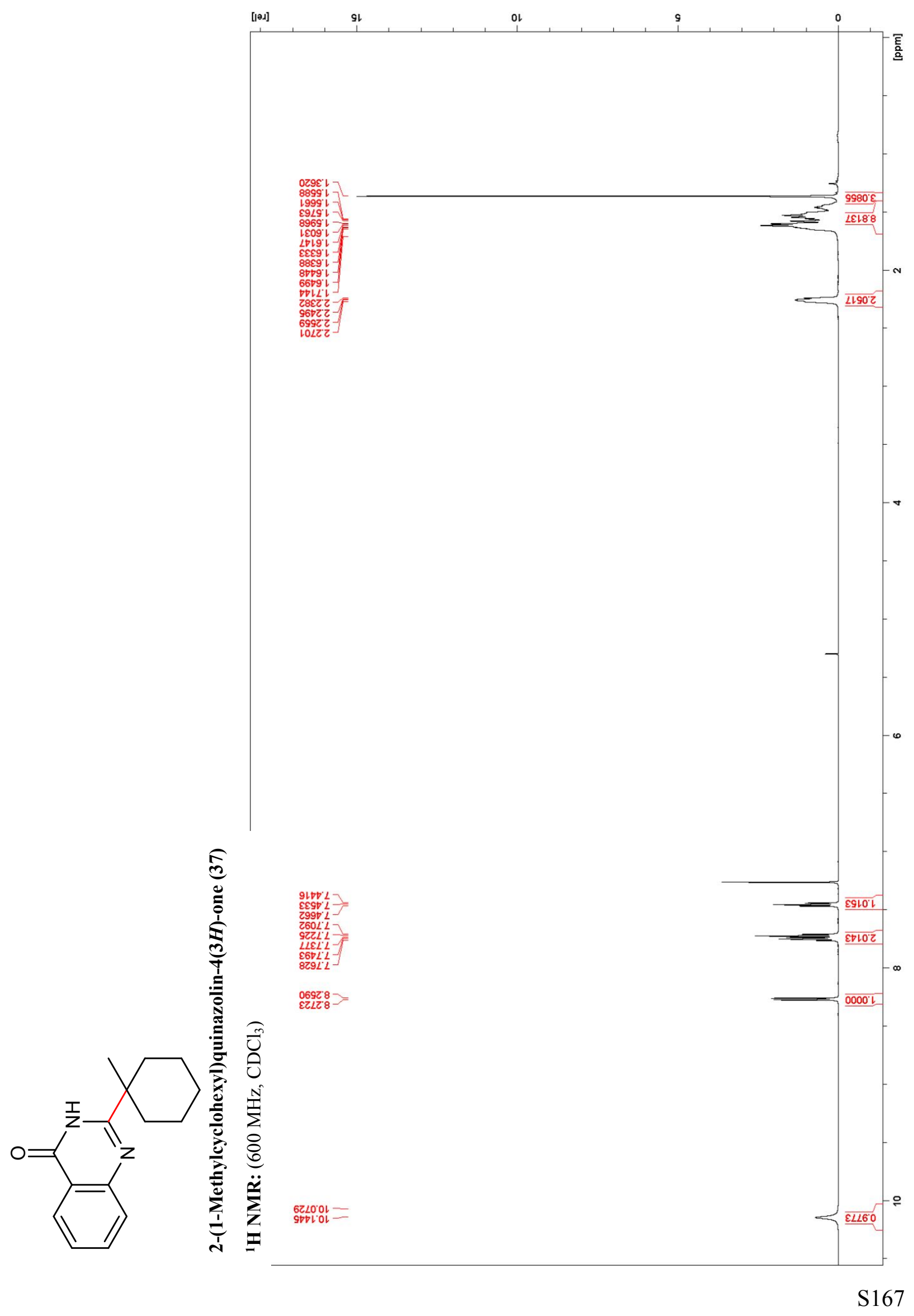




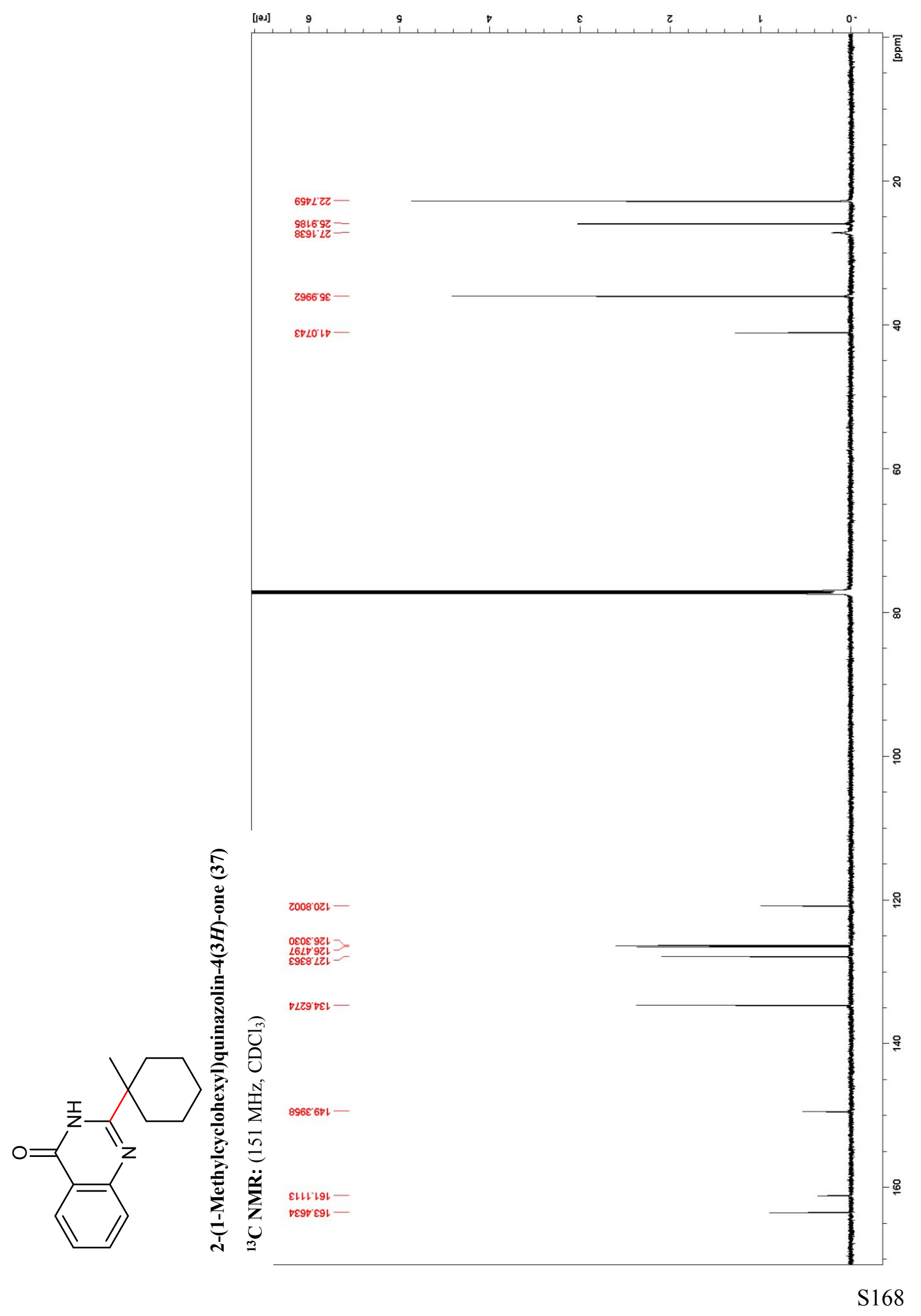




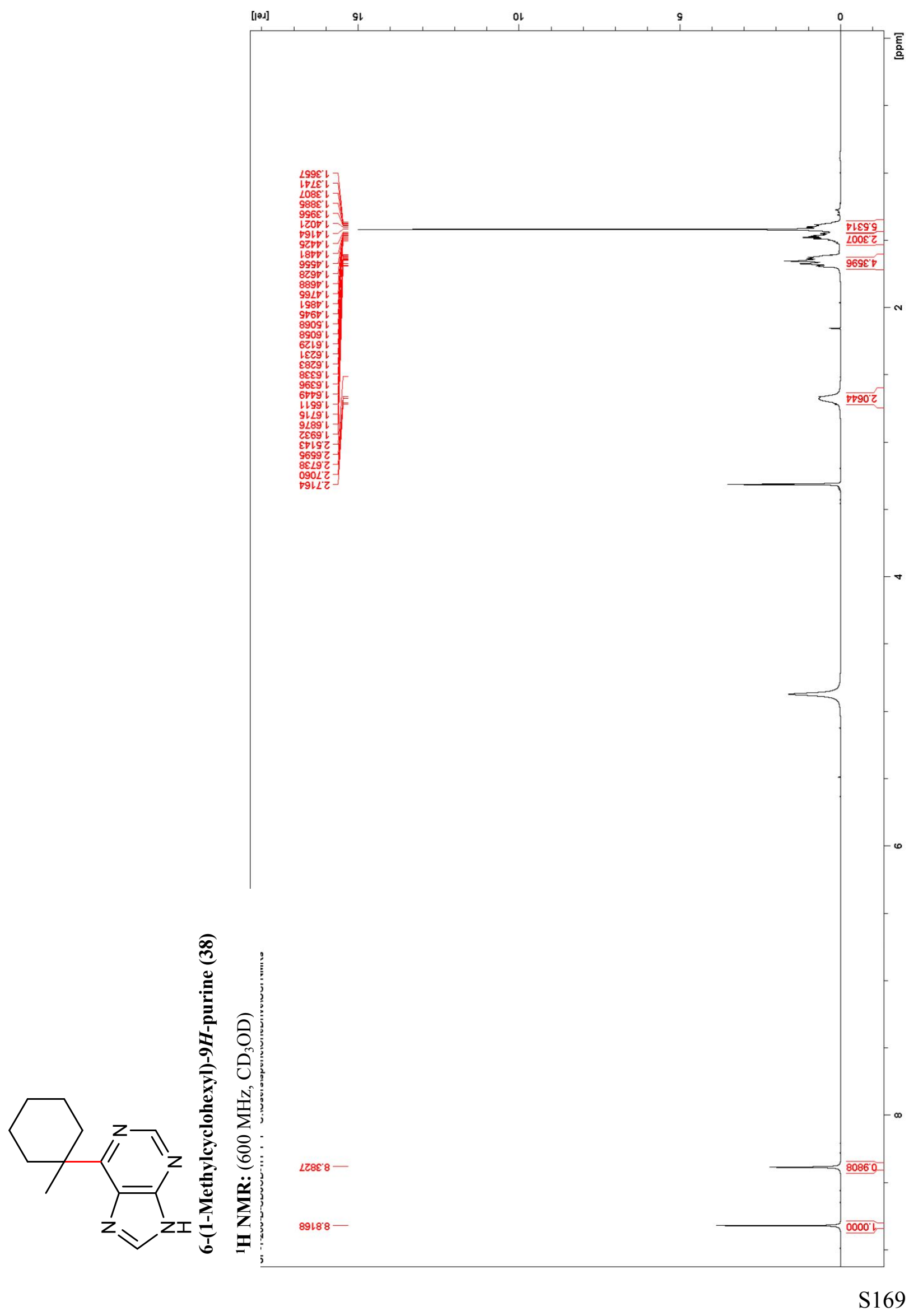




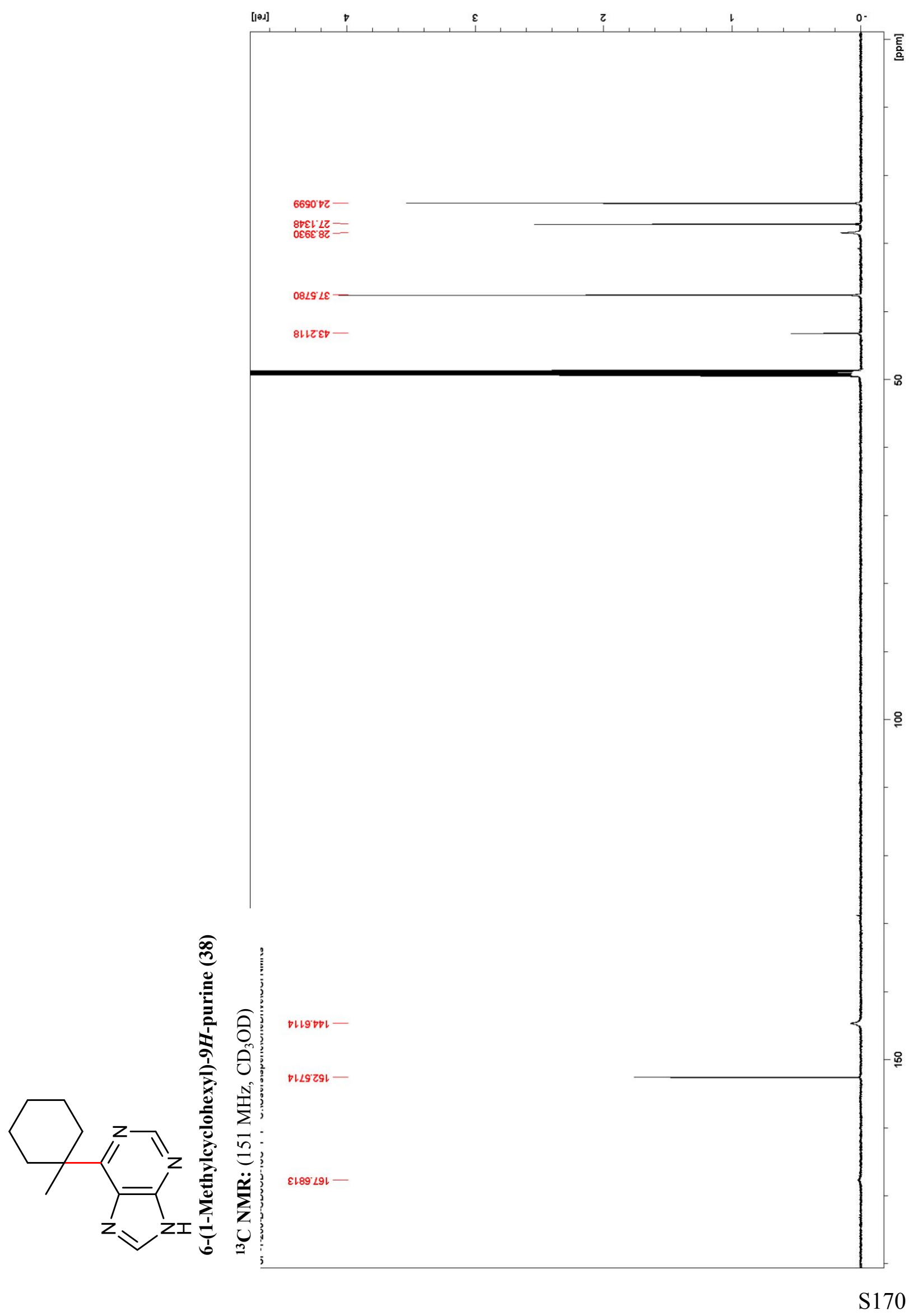




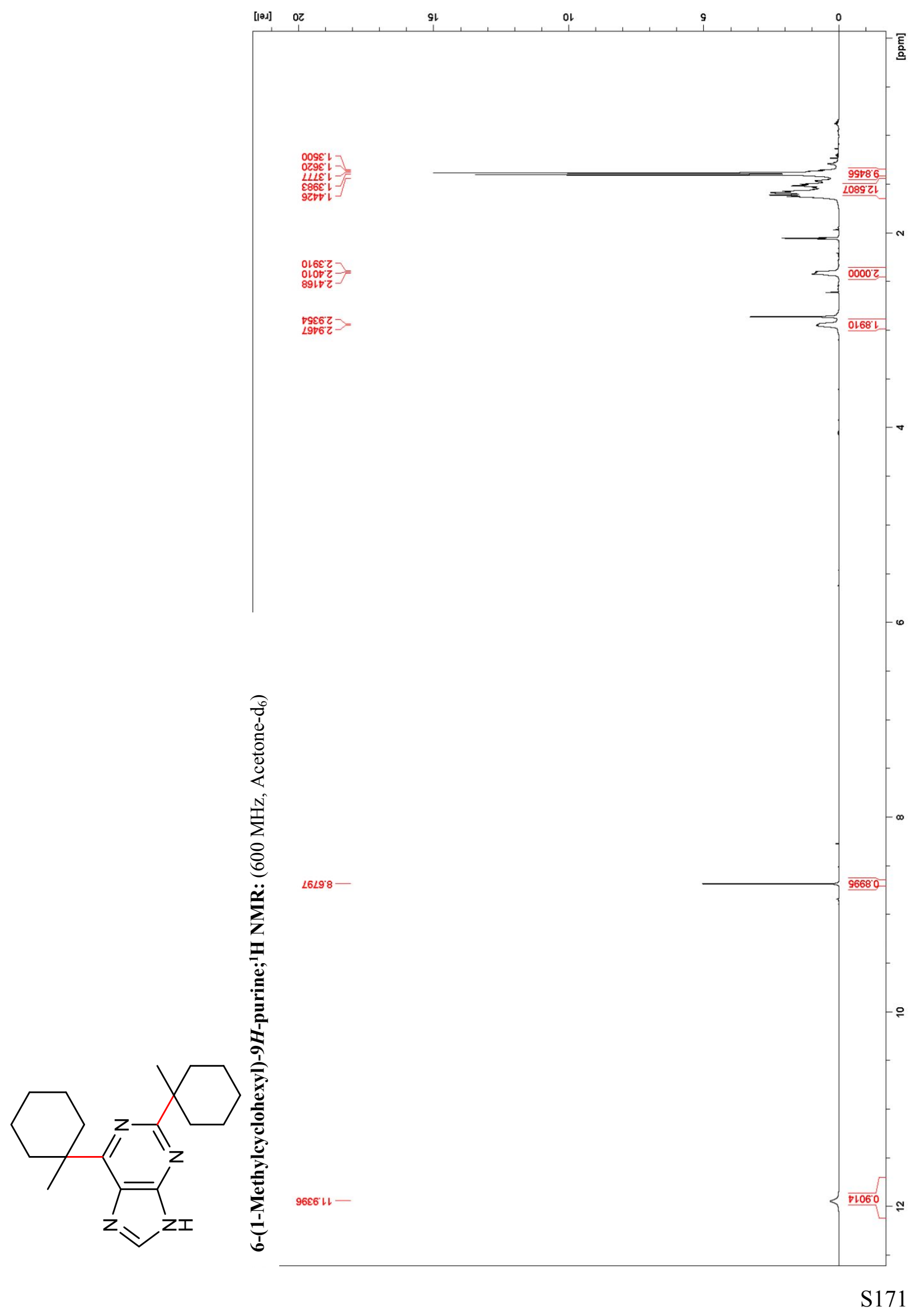




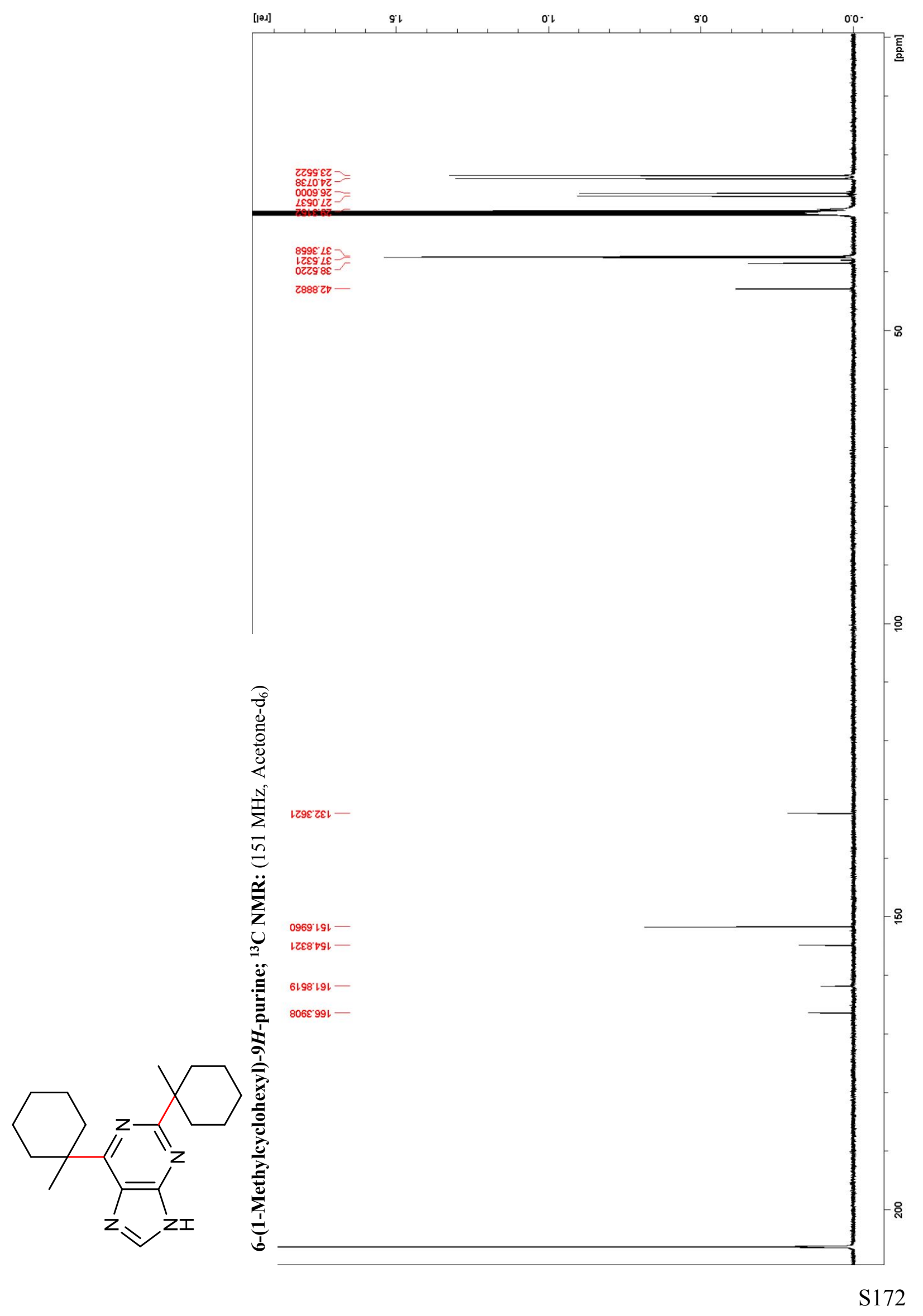




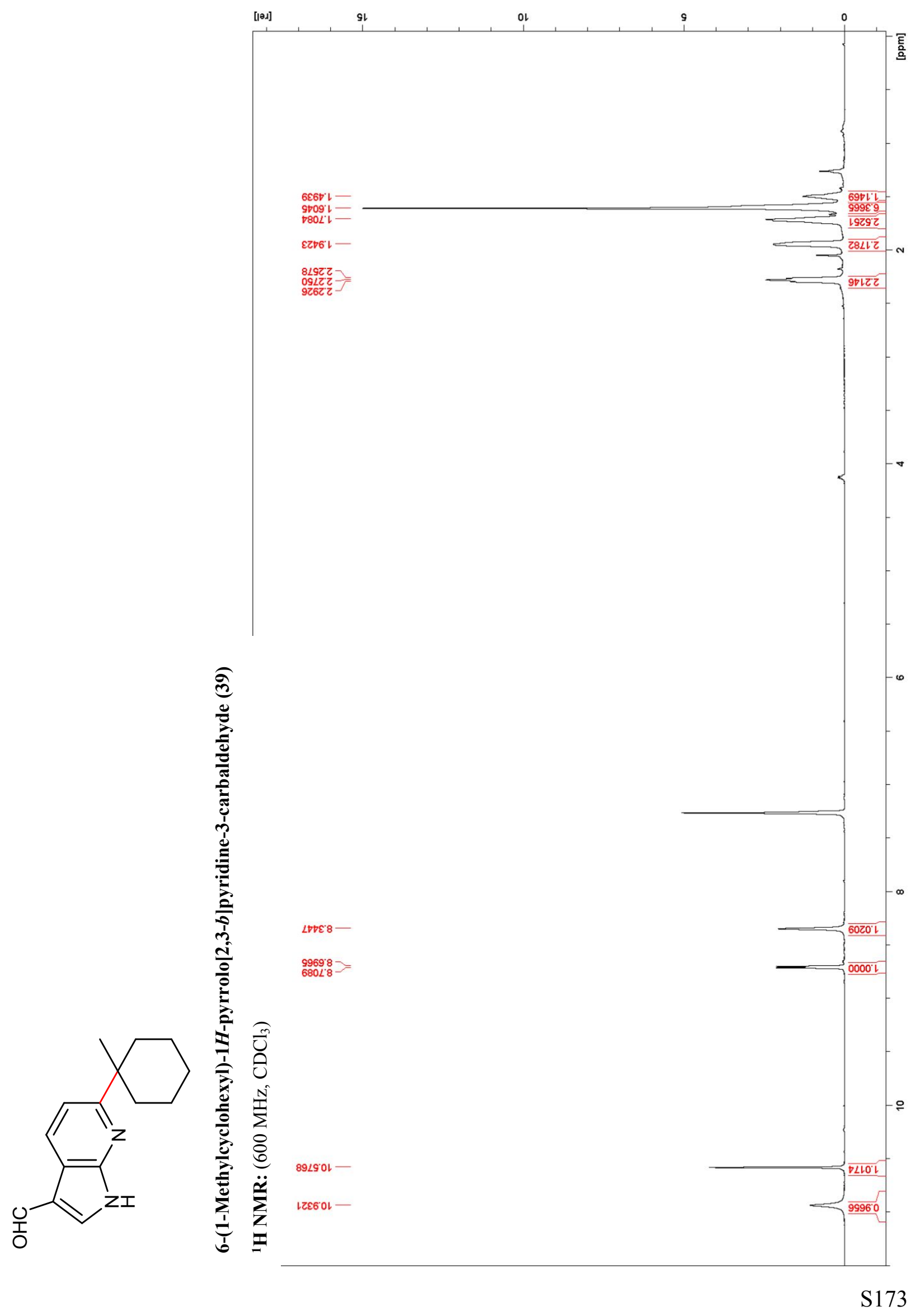




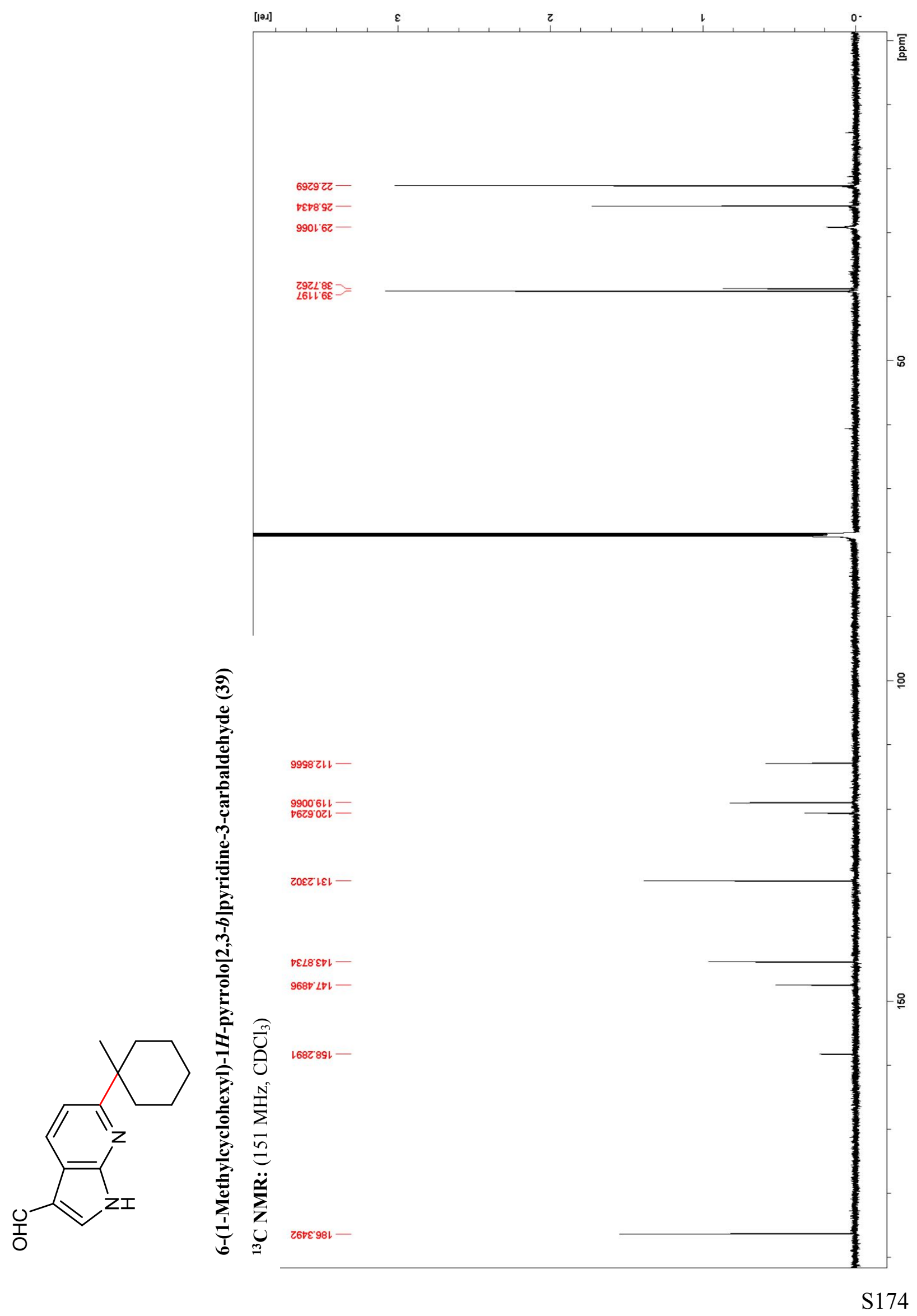




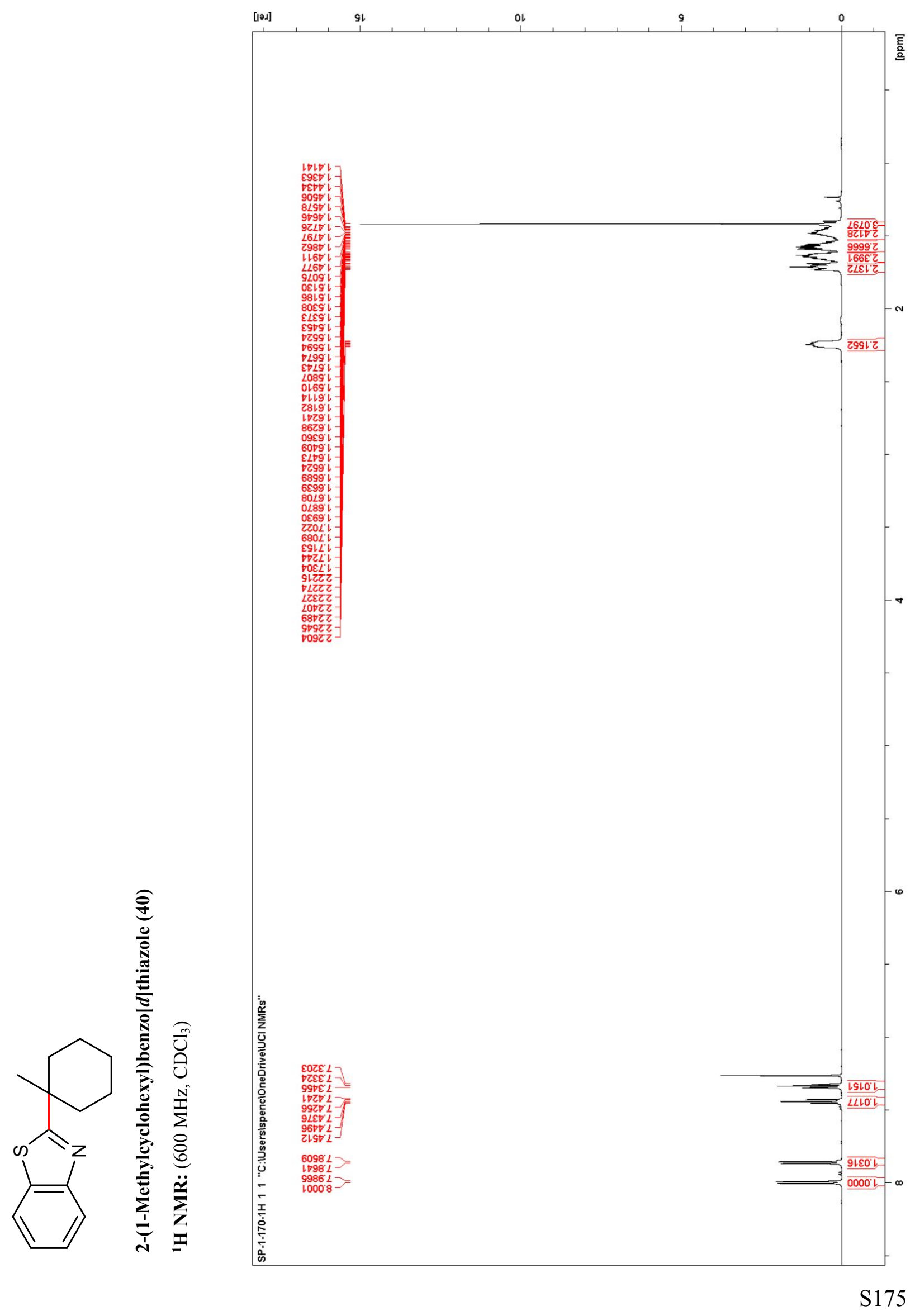




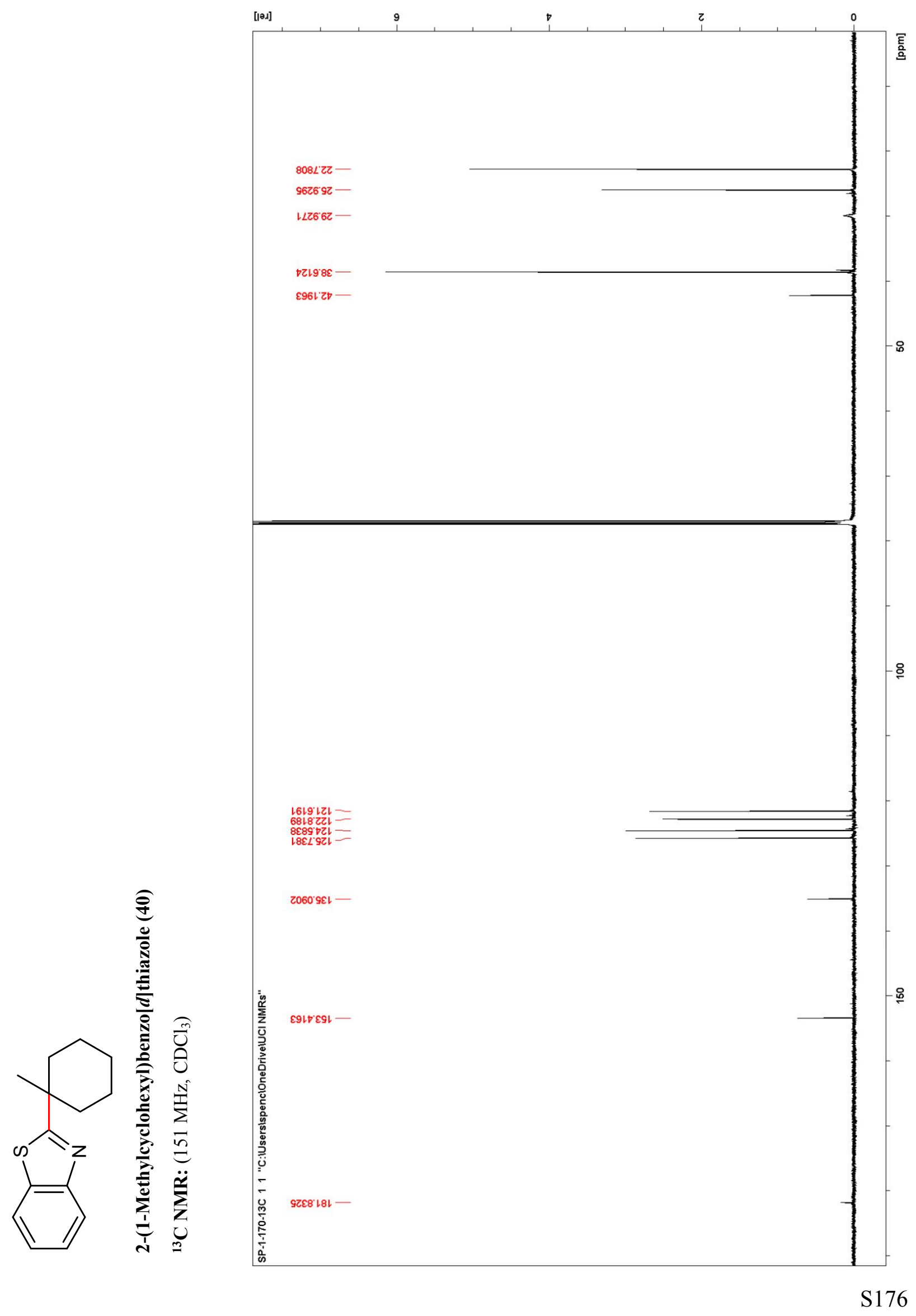




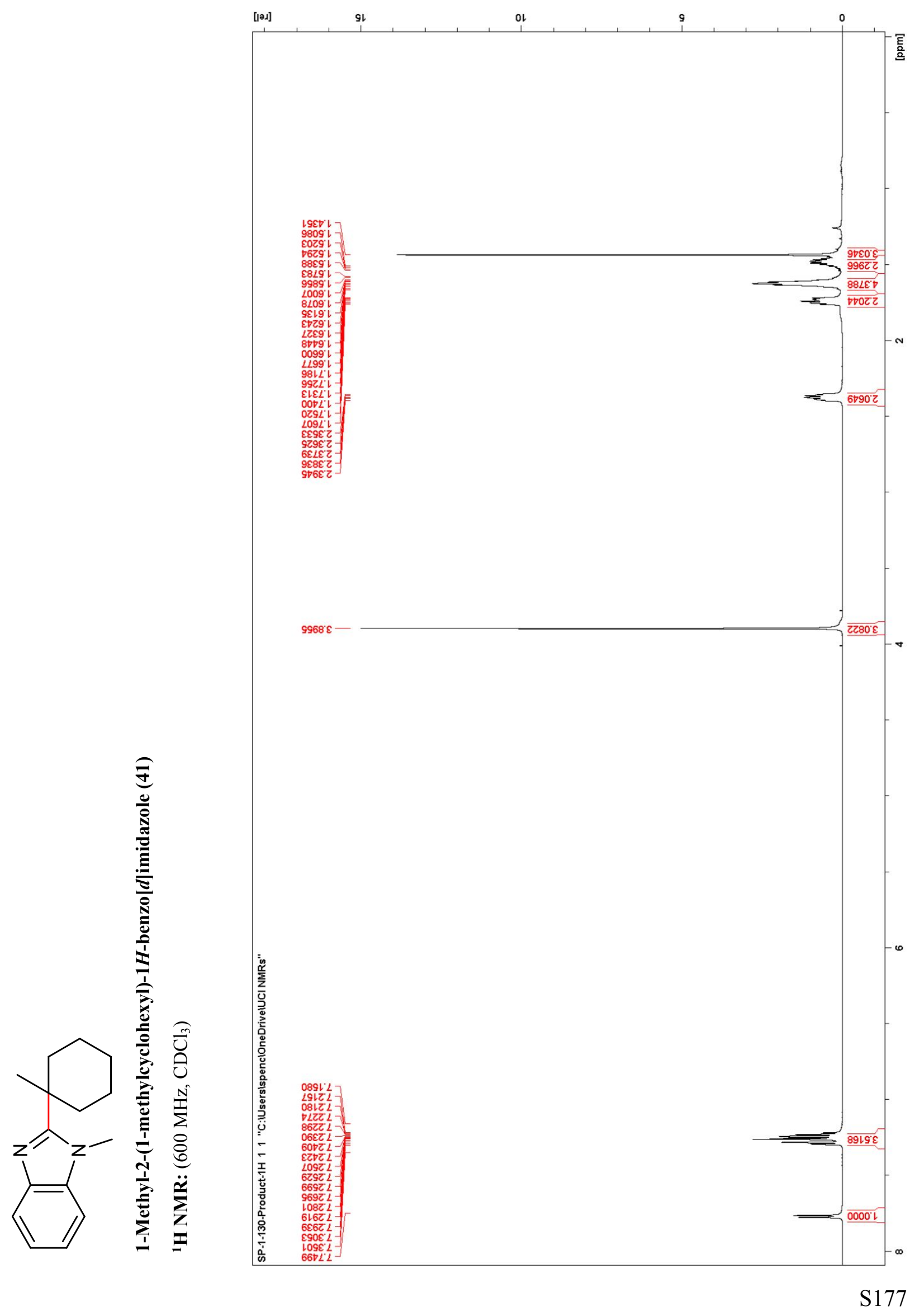




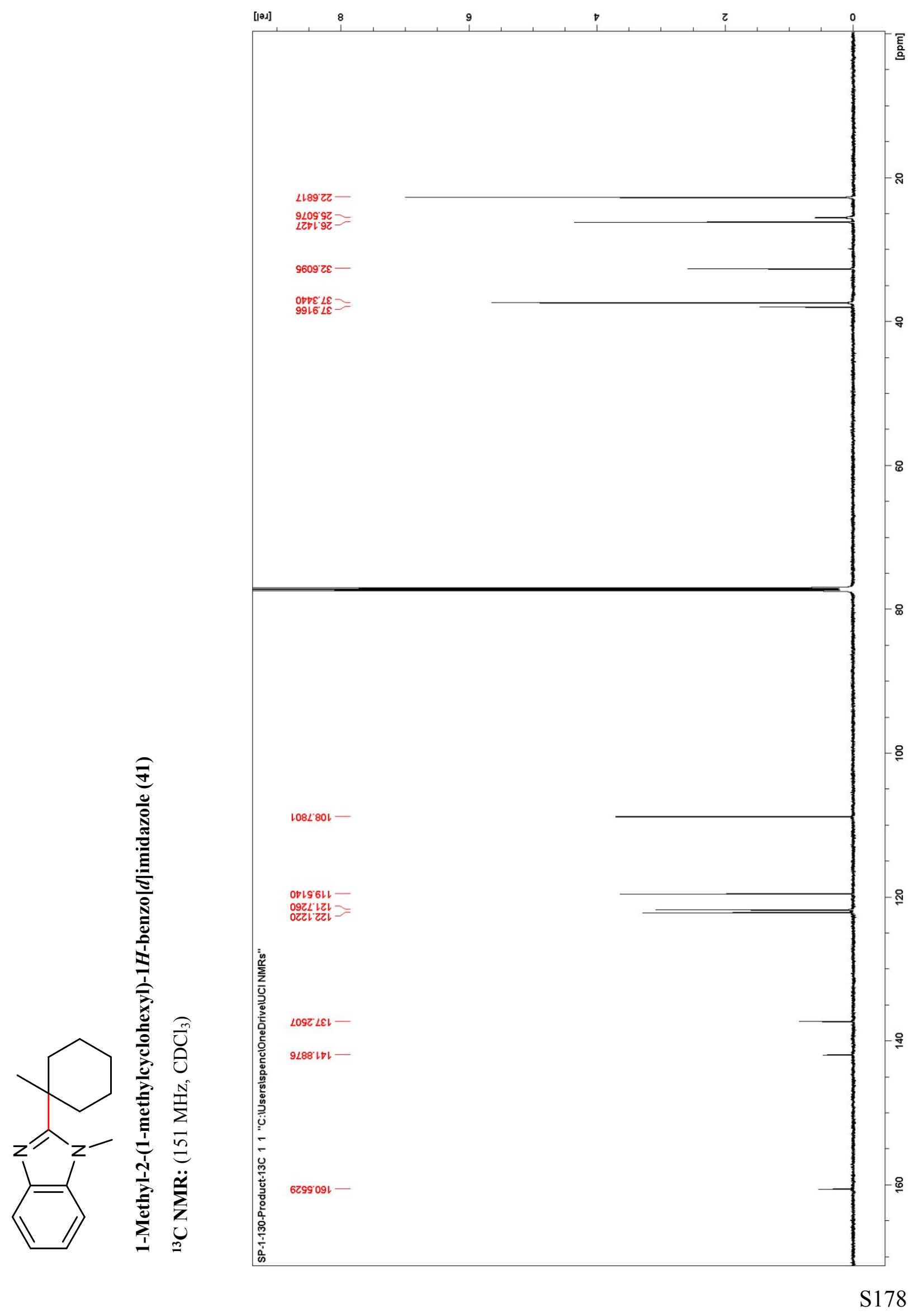




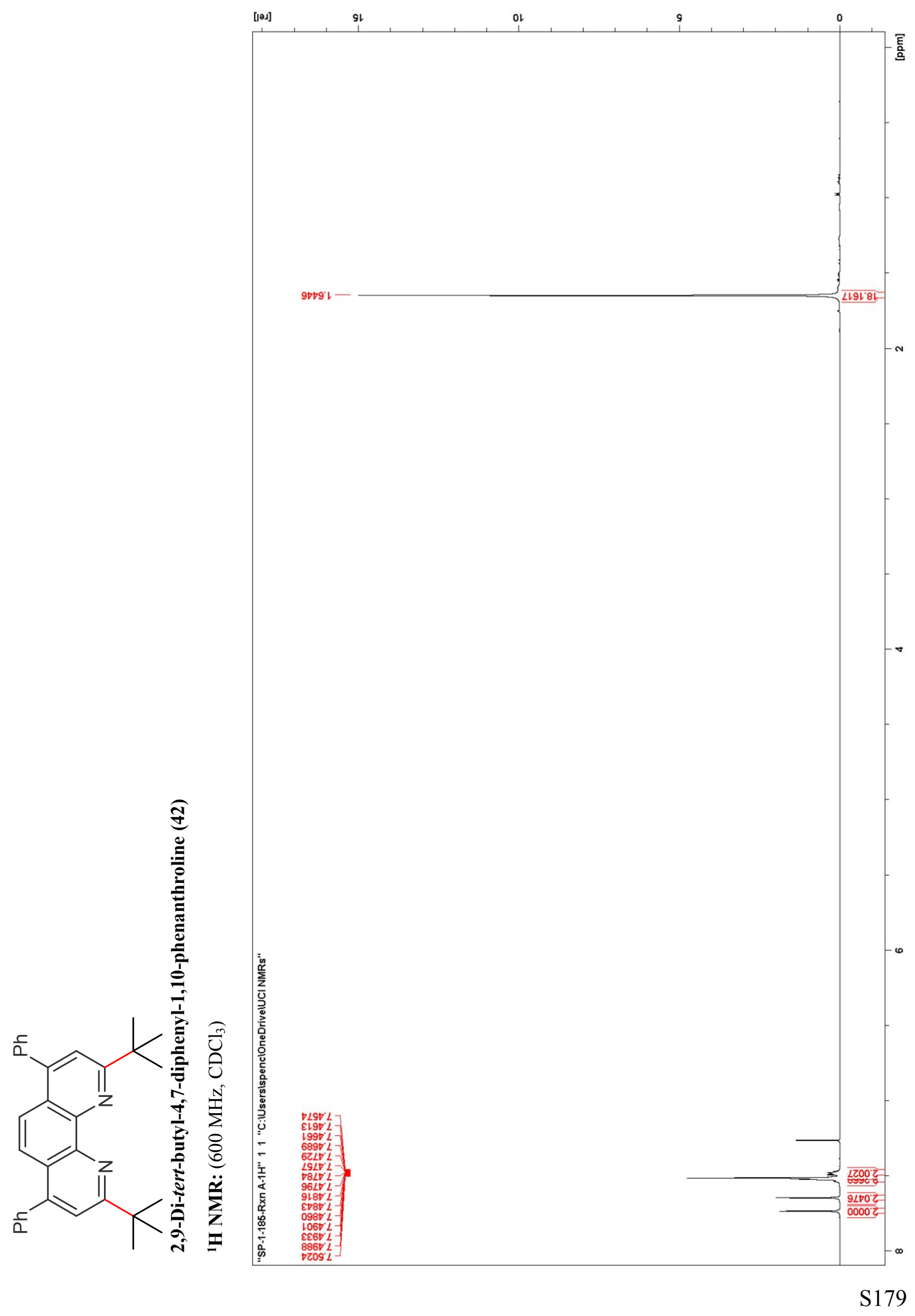




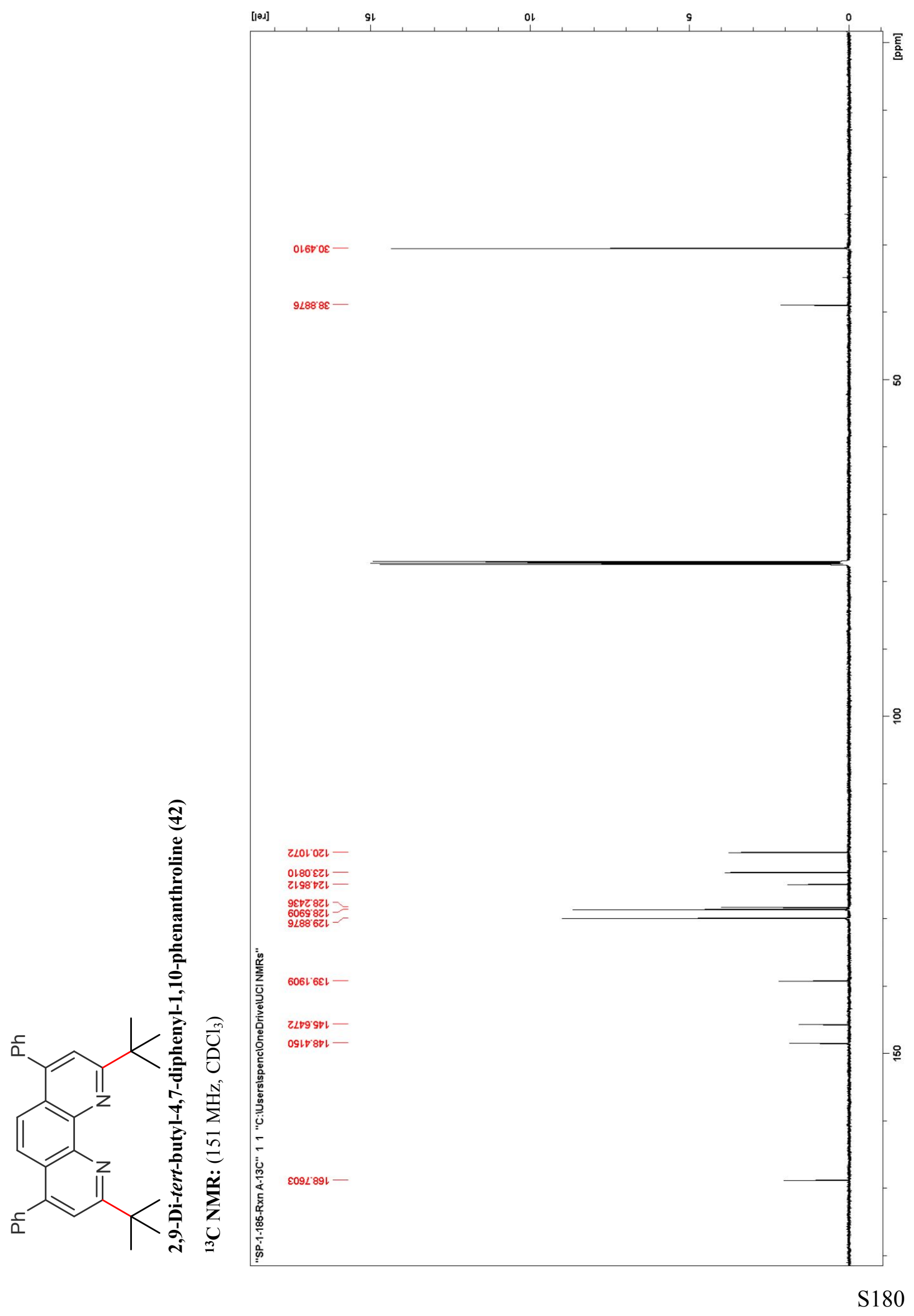




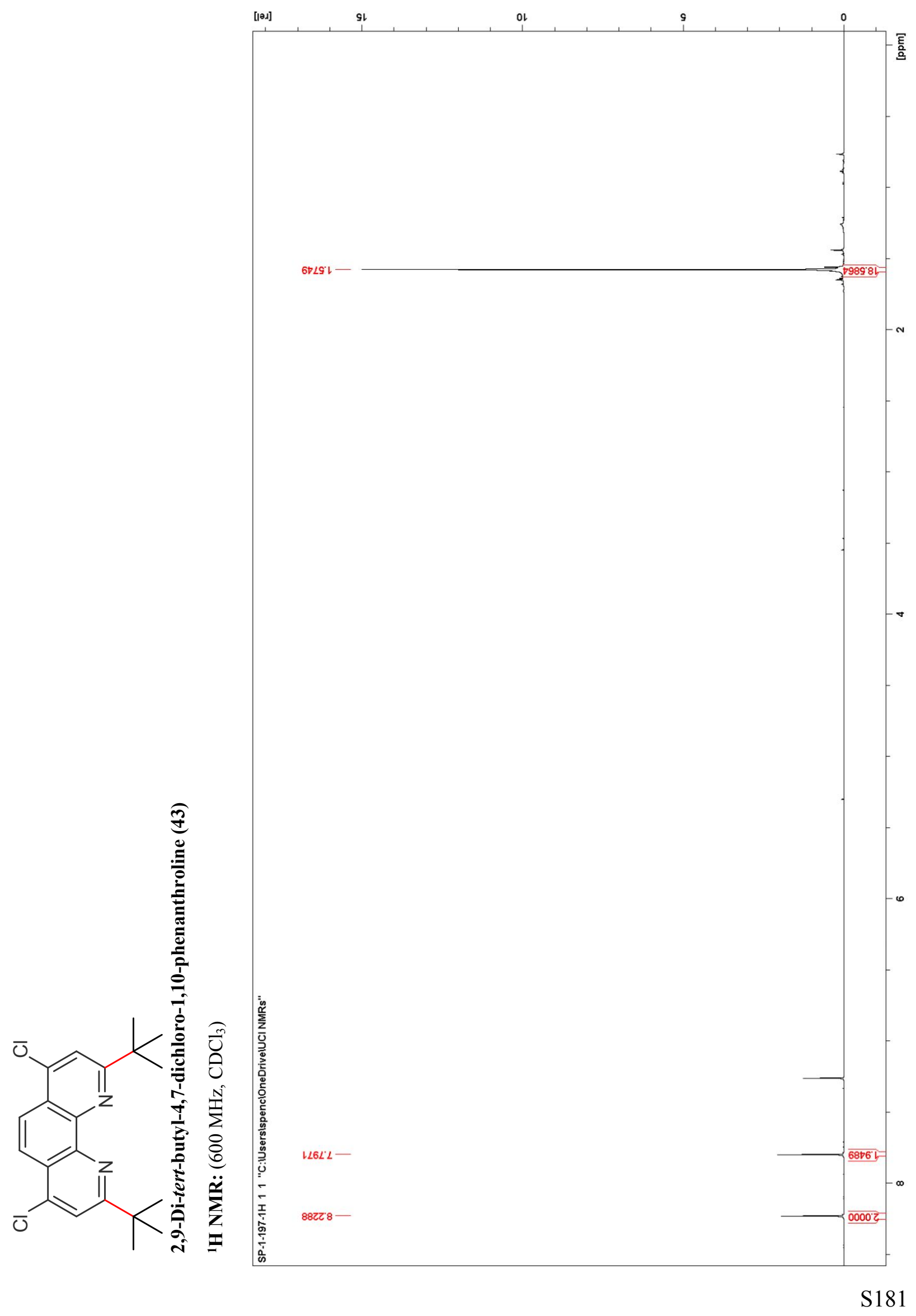




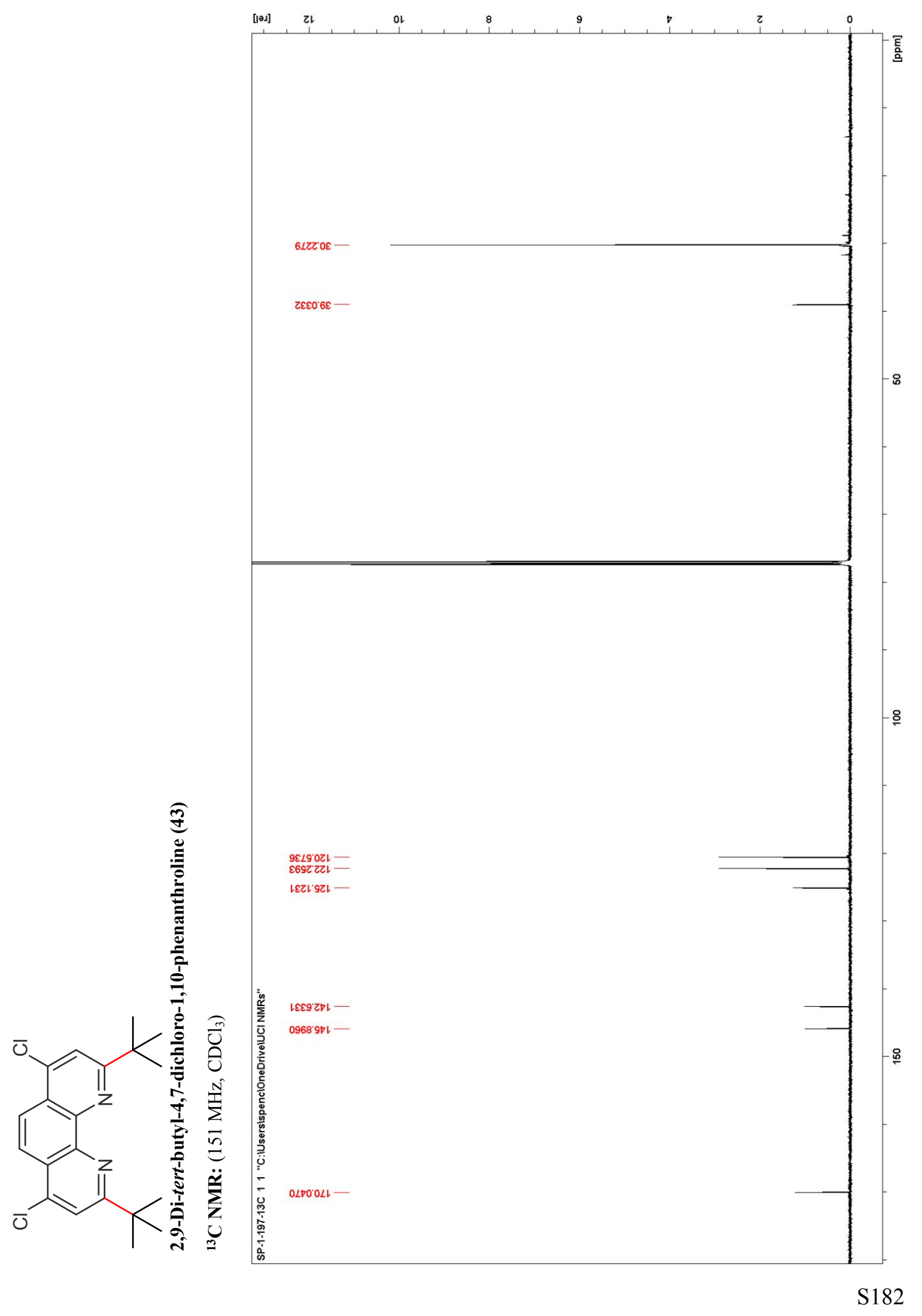




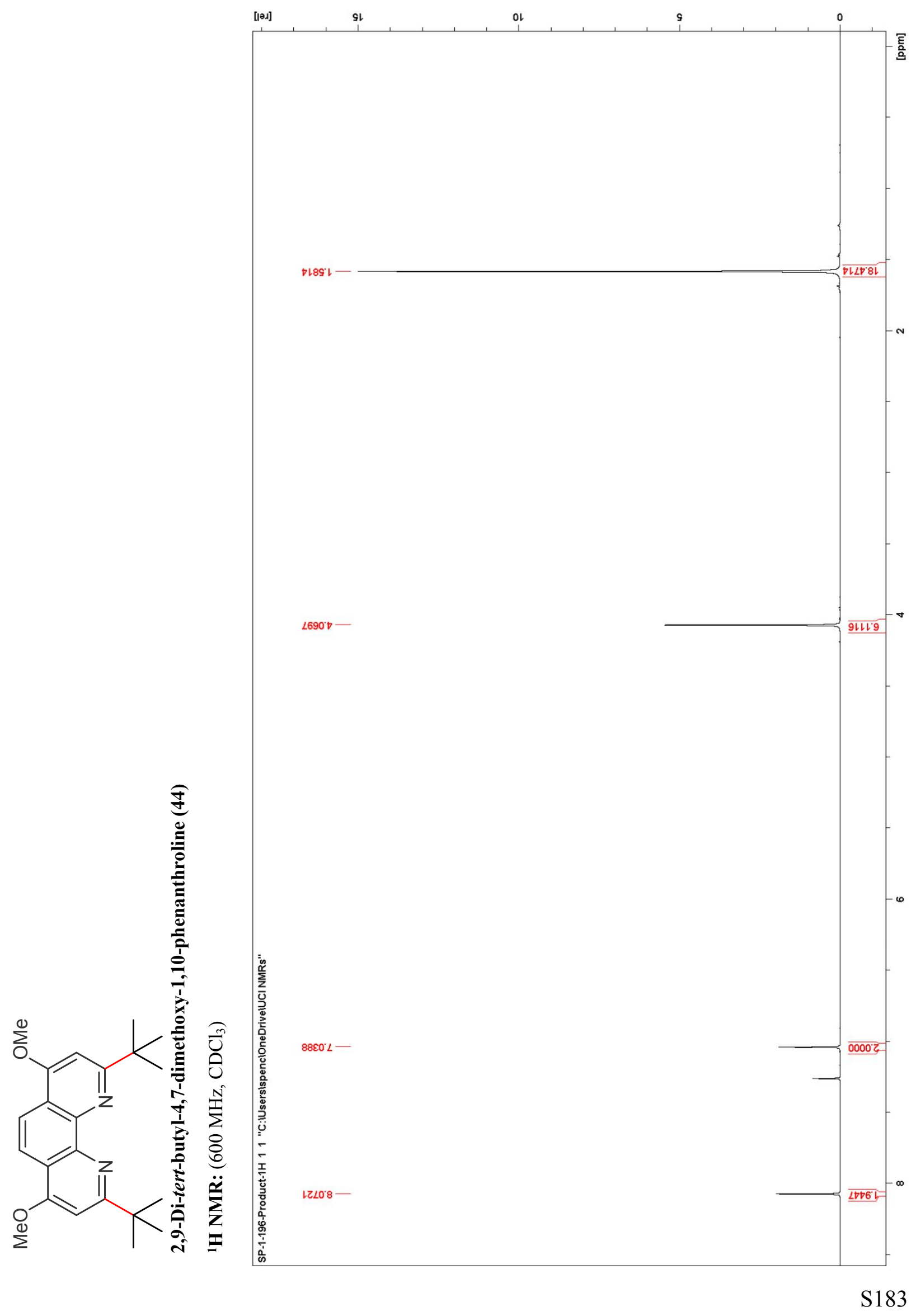




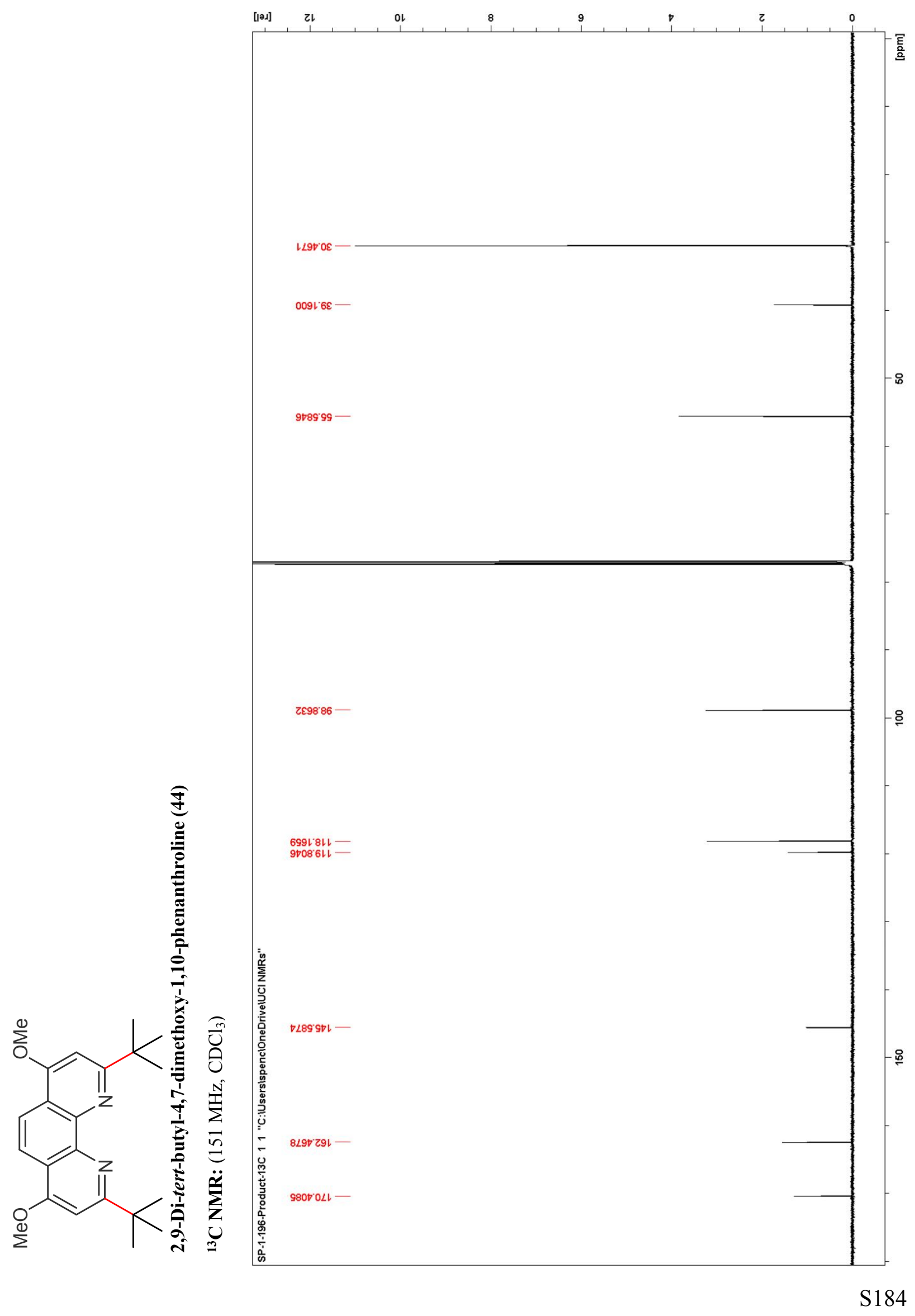




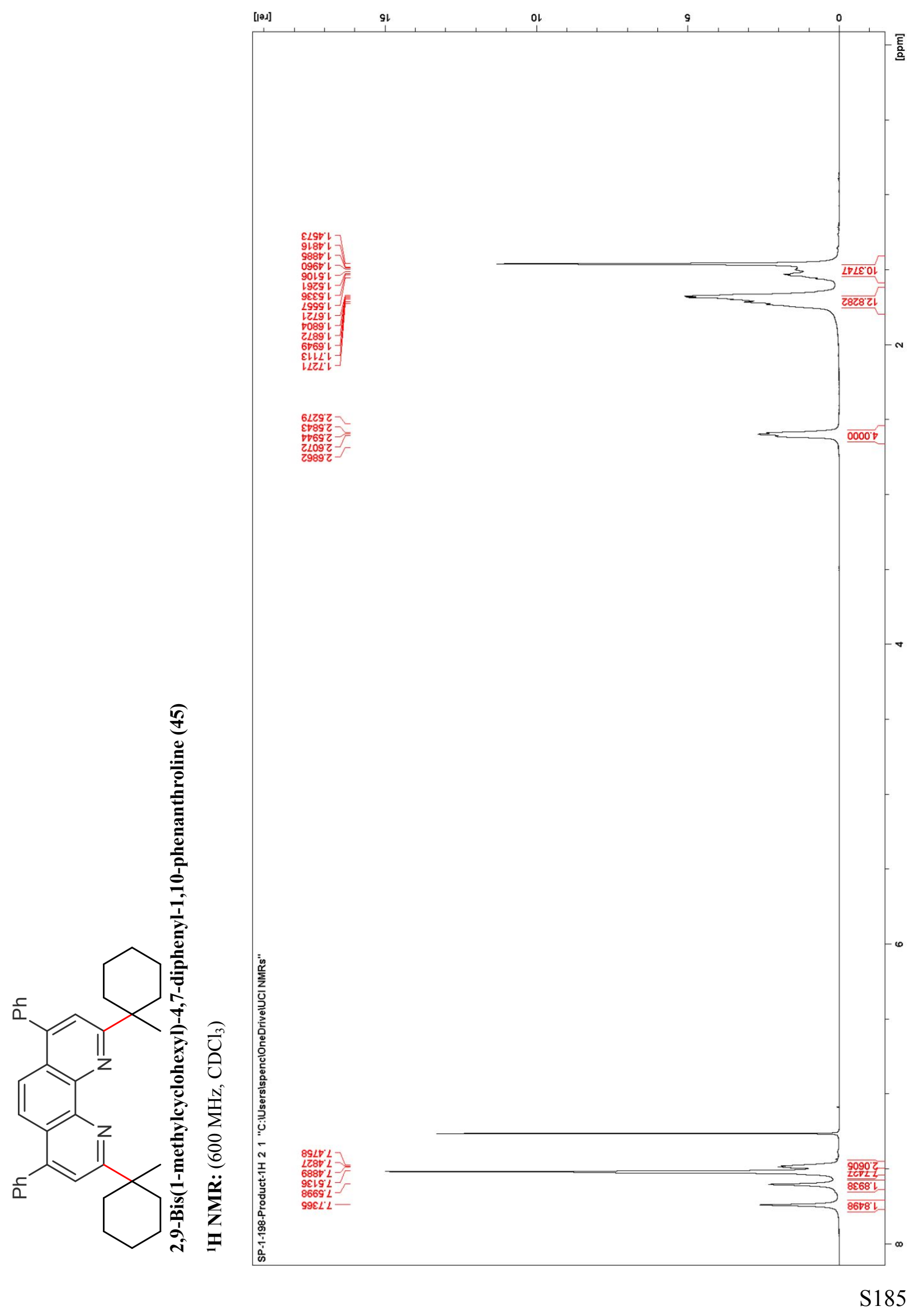




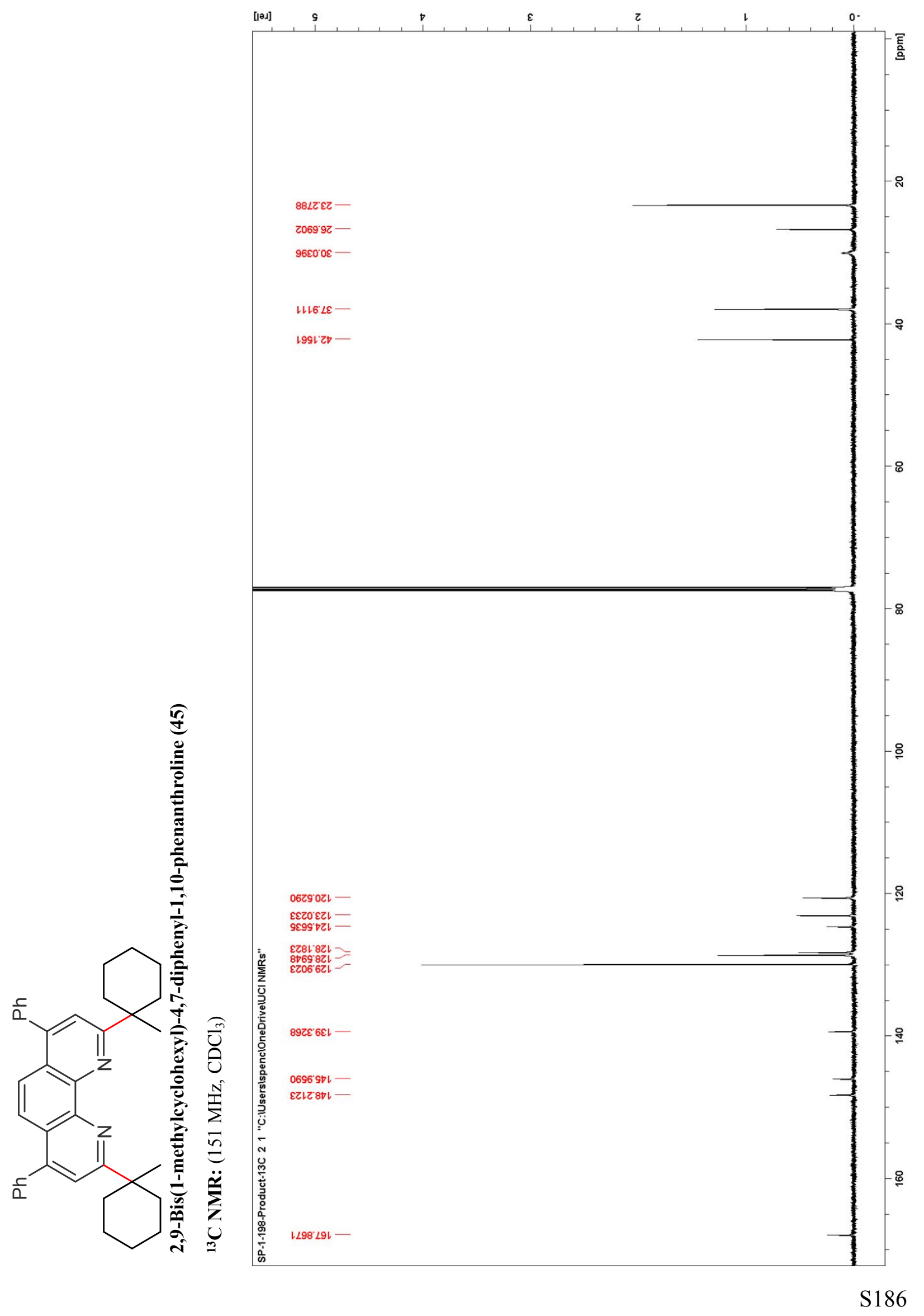




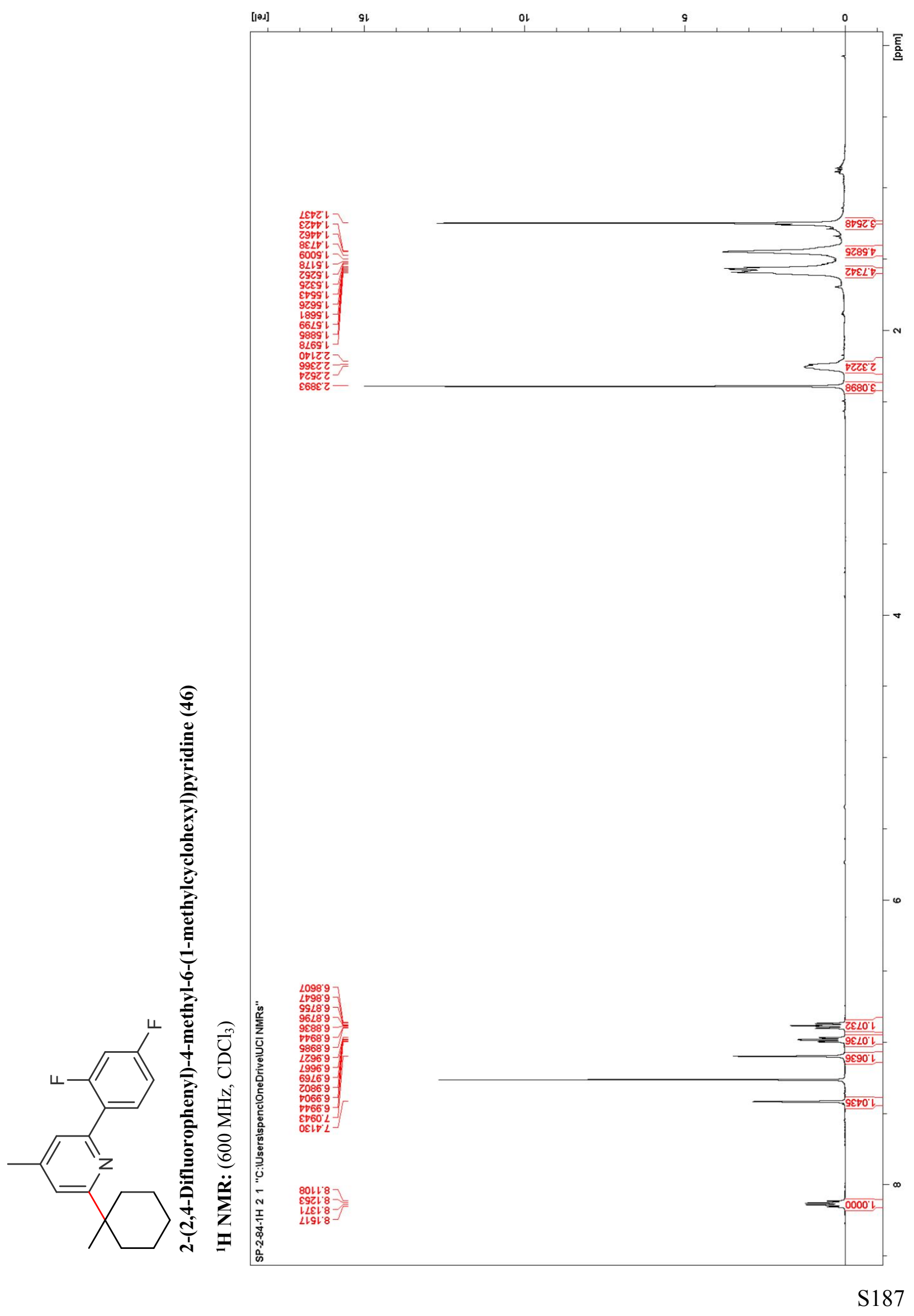




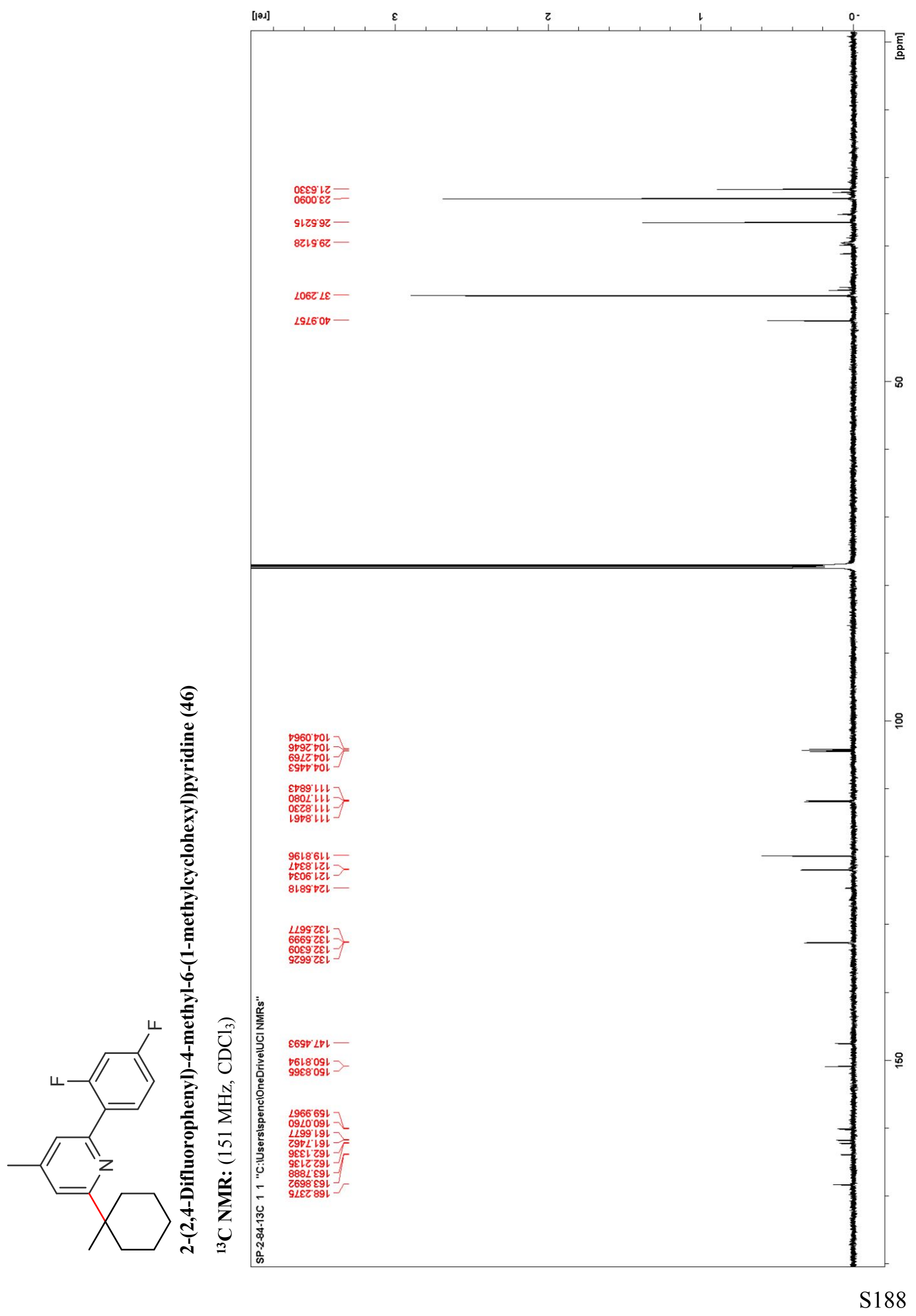




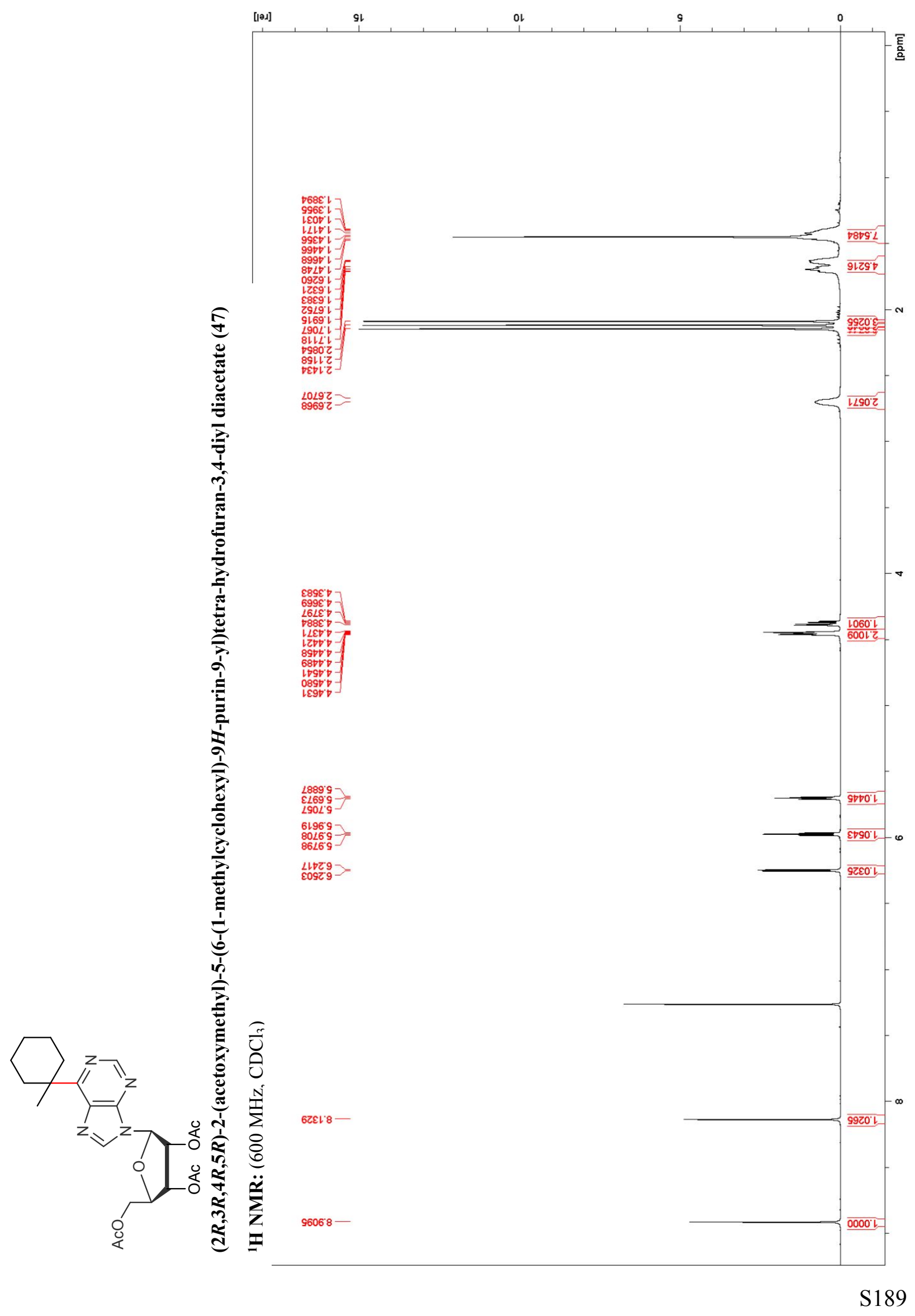




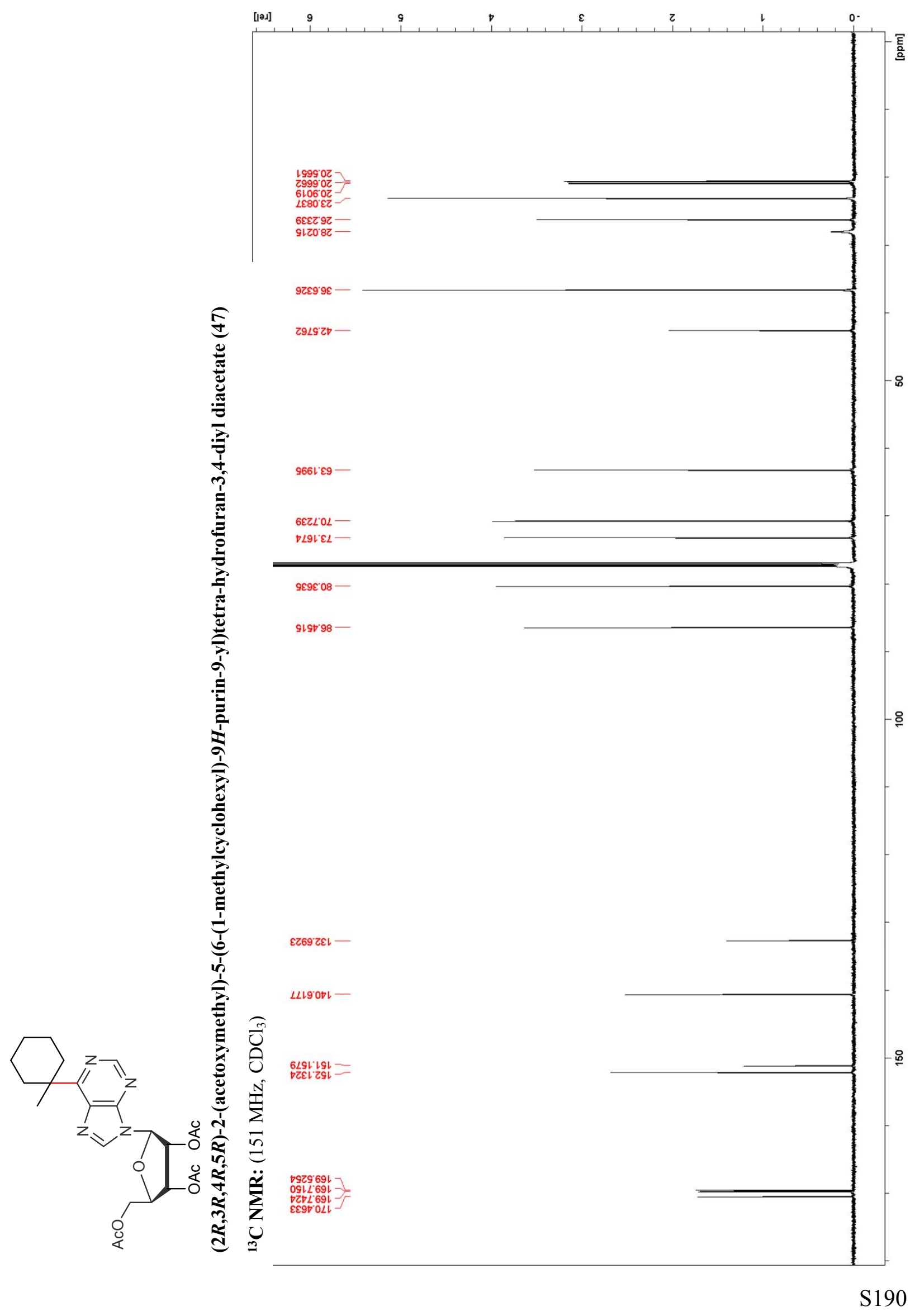




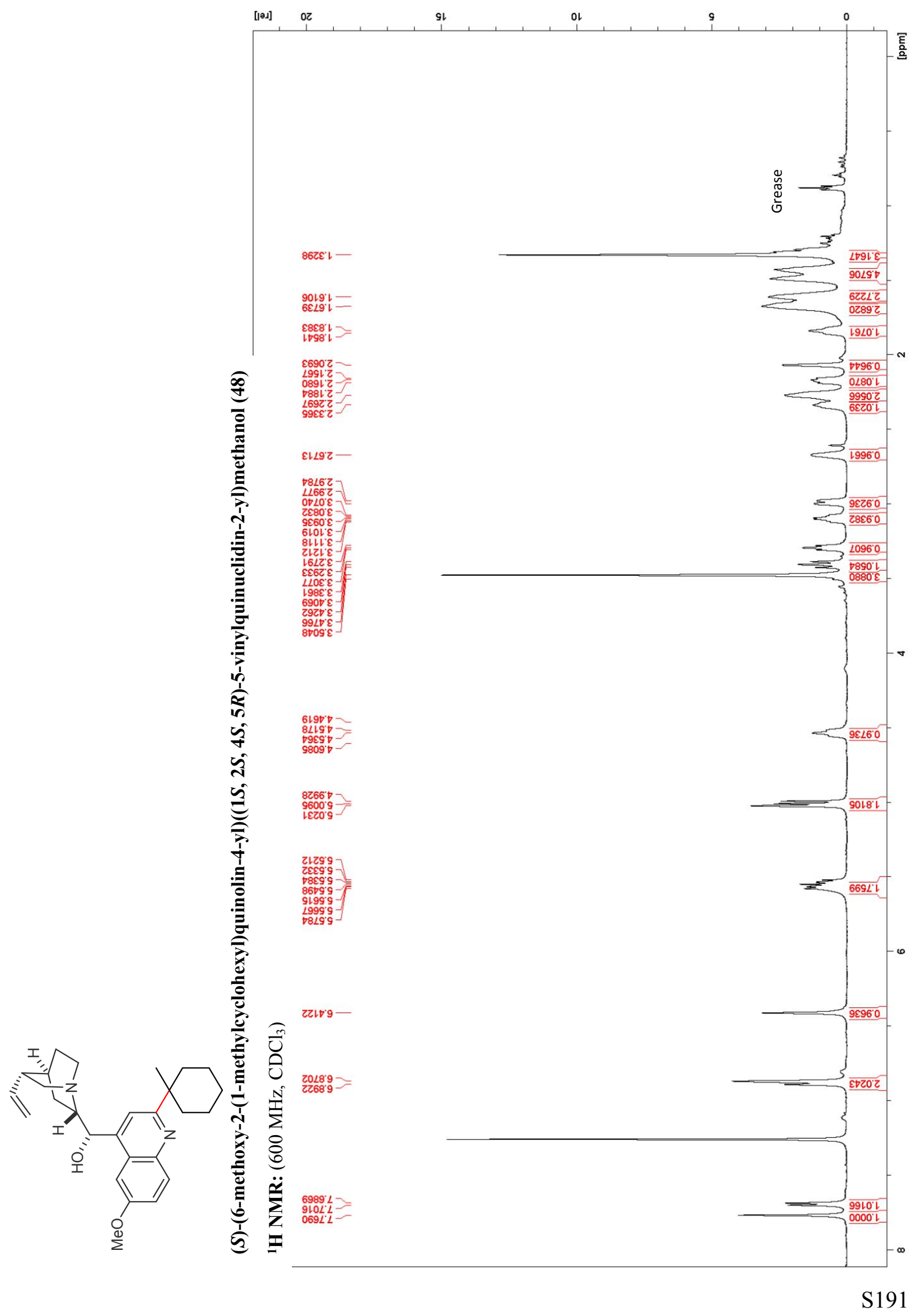




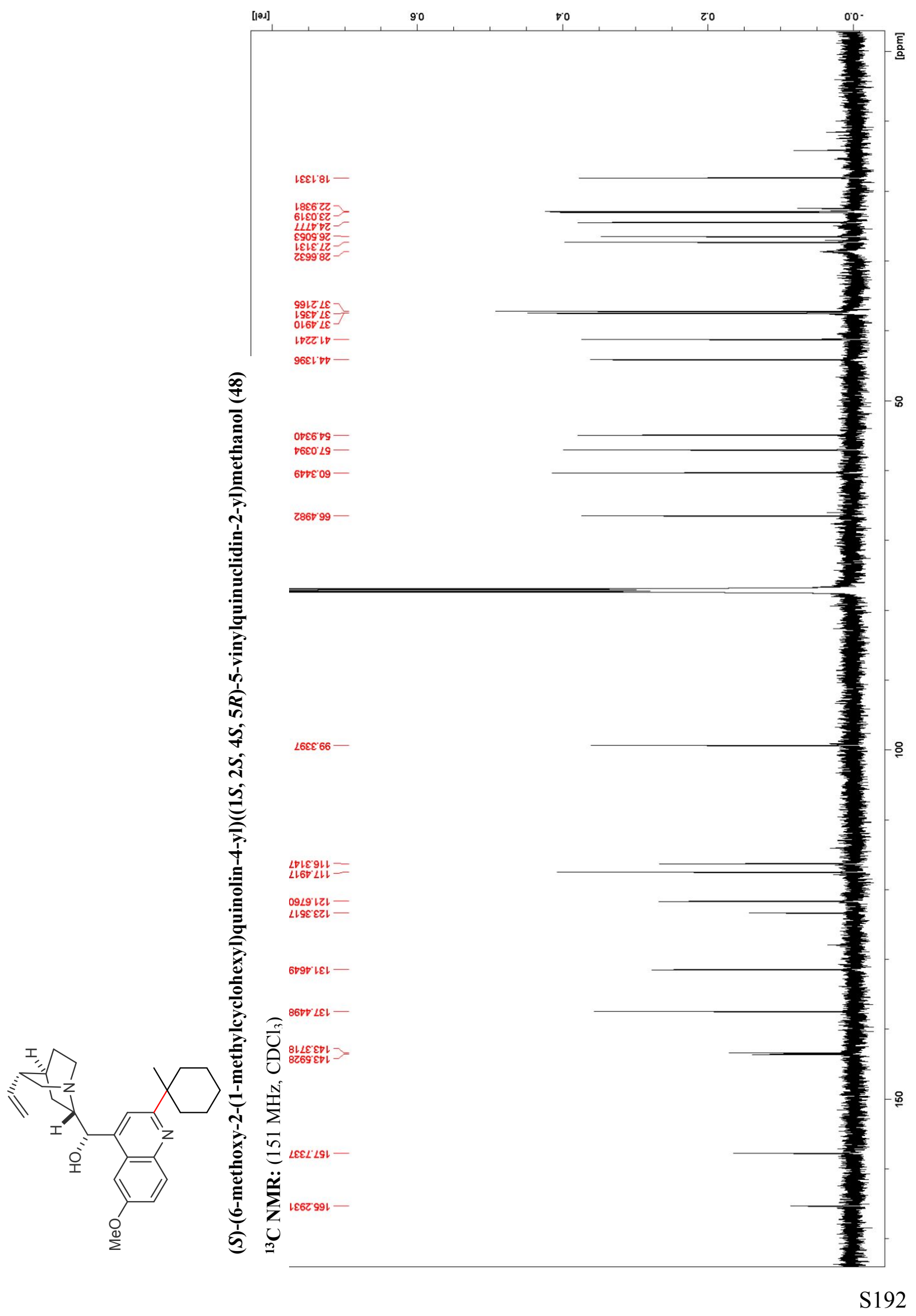




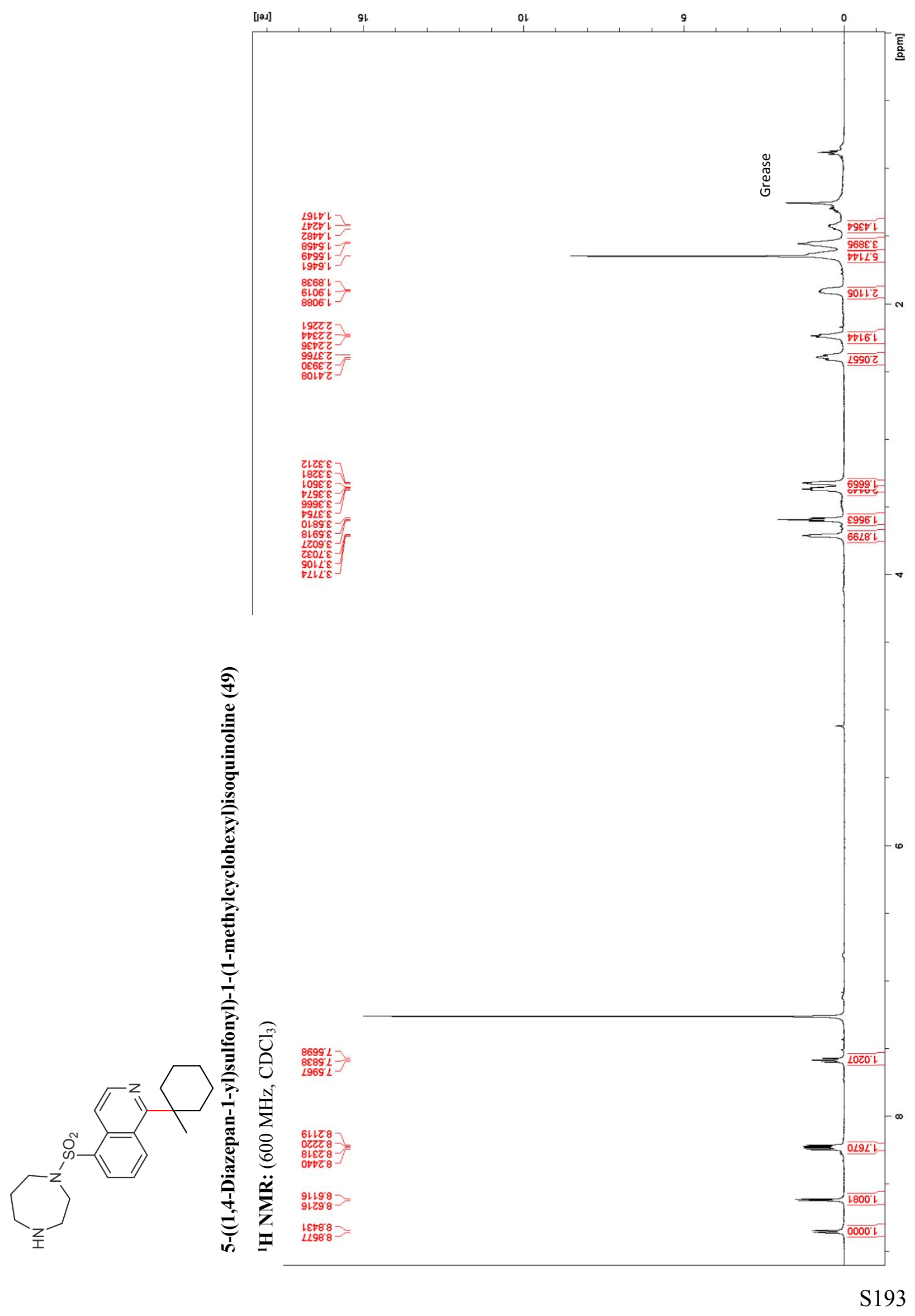




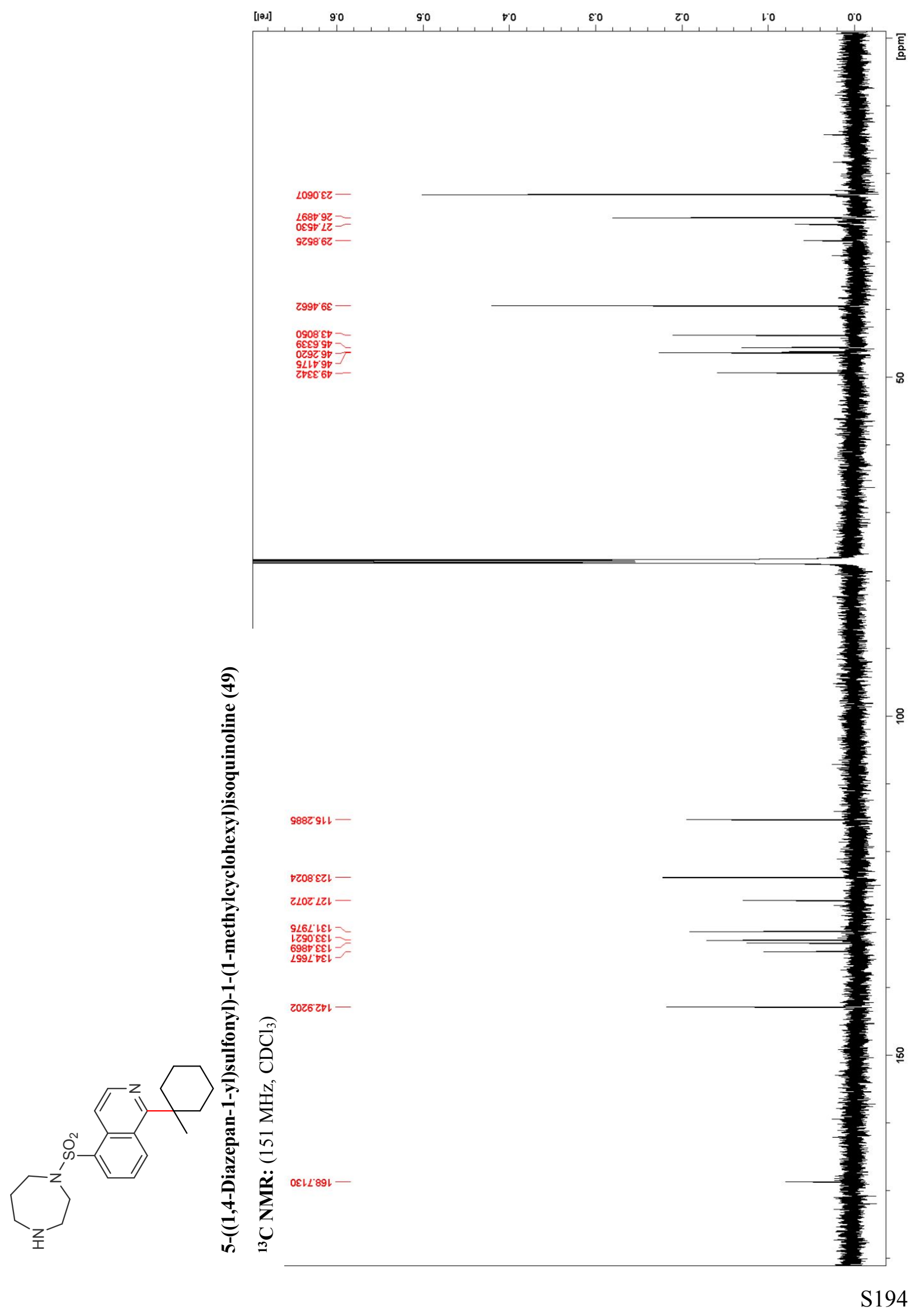

